\title{
Métodos de penalidade e barreira para programação convexa semidefinida
}

\author{
Antonio Carlos dos Santos \\ DissertaÇÃo APRESEnTAdA \\ $\mathrm{AO}$ \\ Instituto de Matemática e Estatí́stica \\ DA \\ Universidade De SÃo PaUlo \\ PARA \\ OBTENÇÃO DO TÍTULO \\ $\mathrm{DE}$ \\ Mestre em Ciências \\ Programa: Ciência da Computação \\ Orientador: Prof. Dr. Paulo José da Silva e Silva \\ Durante o desenvolvimento deste trabalho, o autor recebeu \\ auxílio financeiro da FAPESP - Processo Nº5/57619-6
}

São Paulo, agosto de 2009. 


\title{
Métodos de penalidade e barreira para programação convexa semidefinida
}

\author{
Este exemplar corresponde à redação \\ final da dissertação devidamente corrigida \\ e defendida por Antonio Carlos dos Santos \\ e aprovada pela Comissão Julgadora.
}

Banca Examinadora:

- Prof. Dr. Paulo José da Silva e Silva (orientador) IME - USP.

- Prof. Dr. Ernesto Julian Goldberg Birgin IME - USP.

- Profa. Dra. Fernanda Maria Pereira Raupp PUC - RJ. 


\section{Agradecimentos}

Aos meus pais, Antonio (em memória) e Tereza, por formarem meu caráter com valores essenciais, como honestidade, responsabilidade e dedicação, e pelo grande esforço que fizeram para que minha irmã e eu tivêssemos uma boa educação.

Ao Paulo, pelos ensinamentos e pela orientação que ampliaram muito meu conhecimento e gosto pela Matemática e pela Computação, em particular, pela Otimização. Além disso, pelos conselhos que me ajudaram a crescer como pessoa.

À minha irmã, Juliana, por ter me estimulado o hábito da leitura desde criança.

Às minhas madrinhas Cida e Paulina pelo apoio e carinho.

Aos vários professores que tive até hoje, que estimularam minha curiosidade para continuar aprendendo. Especialmente, ao Carlos Humes, Ernesto Birgin, Paulo Feofiloff, Coelho e Yoshiko.

Ao pessoal da salinha da pós-graduação do IME-USP: Ellen Hidemi, Cardonha, Francisco, Marcel, Lobato, Marina, Márcio, Hashimoto, Cris, Soares, Domingos, Ricardo e Wanderley.

Aos amigos que conheci ao longo dos anos e que foram fundamentais em vários momentos da minha vida: Monique, Carol, Fernando, Bento, Nelson, Onishi, Ricardo (Tio), Edson (em memória), Ana Paula, Lu, Tiago, Tio Du, Zé Luís, Íris, Kleysson, Larizza, Filipe, entre tantos outros.

E a Deus, pelas pessoas e possibilidades que pôs em minha vida e por proteger e guiar meu caminho sempre. 


\section{Resumo}

Este trabalho insere-se no contexto de métodos de multiplicadores para a resolução de problemas de programação convexa semidefinida e a análise de suas propriedades através do método proximal aplicado sobre o problema dual.

Nosso foco será uma subclasse de problemas de programação convexa semidefinida com restrições afins, para a qual estudaremos relações de dualidade e condições para a existência de soluções dos problemas primal e dual.

Em seguida, analisaremos dois métodos de multiplicadores para resolver essa classe de problemas e que são extensões de métodos conhecidos para programação não-linear. O primeiro, proposto por Doljansky e Teboulle, aborda um método de ponto proximal interior entrópico e sua conexão com um método de multiplicadores exponenciais. O segundo, apresentado por Mosheyev e Zibulevsky, estende para a classe de problemas de nosso interesse um método de lagrangianos aumentados suaves proposto por Ben-Tal e Zibulevsky.

Por fim, apresentamos os resultados de testes numéricos feitos com o algoritmo proposto por Mosheyev e Zibulevsky, analisando diferentes escolhas de parâmetros, o aproveitamento do padrão de esparsidade das matrizes do problema e critérios para a resolução aproximada

dos subproblemas irrestritos que devem ser resolvidos a cada iteração desse algoritmo de lagrangianos aumentados. 


\section{Abstract}

This work deals with multiplier methods to solve semidefinite convex programming problems and the analysis of their proprieties based on the proximal point method applied on the dual problem.

We focus on a subclass of semidefinite programming problems with affine constraints, for which we study duality relations an conditions for the existence of solutions of the primal and dual problems.

Afterwards, we analyze two multiplier methods to solve this class of problems which are extensions of known methods in nonlinear programming. The first one, introduced by Doljansky e Teboulle, approaches an entropic interior proximal algorithm and their relationship with an exponential multiplier method. The second one, presented by Mosheyev e Zibulevsky, extends a smooth augmented Lagrangian method proposed by Ben-Tal and Zibulevsky for the problems of our interest.

Finally, we present the results of numerical experiments for the algorithm proposed by Mosheyev e Zibulevsky, analyzing some choices of parameters, the sparsity patterns of matrices of the problem and criteria to accept approximate solutions of the unconstrained subproblems that must be solved at each iteration of the augmented Lagrangian method. 


\section{Sumário}

$\begin{array}{ll}\text { Introdução } & 1\end{array}$

1 Conceitos básicos importantes 3

1.1 Matrizes reais . . . . . . . . . . . . . . . . . . 3

1.2 Matrizes simétricas . . . . . . . . . . . . . . . . . 4

1.3 Matrizes semidefinidas positivas . . . . . . . . . . . . . . 6

1.4 Análise convexa . . . . . . . . . . . . . . . . . . . . . 9 9

1.5 Cálculo matricial . . . . . . . . . . . . . . . . . . . . . 13

1.5.1 Funções matriciais primárias . . . . . . . . . . . . . . . 16

2 Apresentação dos problemas $\quad 27$

2.1 O problema primal . . . . . . . . . . . . . . . 27

2.2 O problema dual . . . . . . . . . . . . . . . . . . . . . 28

2.2 .1 Relações de dualidade . . . . . . . . . . . . . . . . . 30

2.3 Dualidade forte e condições de otimalidade . . . . . . . . . . . . . . 33

3 Um algoritmo proximal interior e o método de multiplicadores exponen$\begin{array}{ll}\text { ciais para programação semidefinida } & 41\end{array}$

3.1 Algoritmo de ponto proximal interior . . . . . . . . . . . . . . . . . 41

3.1.1 Análise de convergência do algoritmo proximal . . . . . . . . . . . 45

3.2 Método de multiplicadores exponenciais para prog. semidefinida . . . . . . 51

4 Métodos de penalidade e barreira para programação semidefinida $\quad 61$

4.1 Algoritmo de penalidade e barreira . . . . . . . . . . . . . . . 61

4.1.1 Escolha da função penalidade/barreira . . . . . . . . . . . . . 66

4.2 Análise dual do algoritmo de penalidade e barreira . . . . . . . . . . . . 67

4.2.1 Conexão do método de lagrangiano aumentado com um algoritmo de ponto proximal . . . . . . . . . . . . . . 70

4.2.2 Considerações sobre o algoritmo de penalidade e barreira . . . . . . 72

4.3 Obtenção de limitantes duais . . . . . . . . . . . . . . . . . . . 74

4.3.1 Obtenção de um limitante dual a partir de um minimizador inexato $\quad 77$

5 Testes numéricos $\quad \mathbf{7 9}$

5.1 Apresentação da implementação feita . . . . . . . . . . . . . . . . . . 79

5.1.1 Parâmetros modificados nos testes . . . . . . . . . . . . . . 80

5.1.2 Escolha da função penalidade e atualização do parâmetro de penalidade 81 
5.1 .3 Critérios de parada . . . . . . . . . . . . . . . . 82

5.1 .4 Ambiente de testes . . . . . . . . . . . . . . 82

5.2 Complexidade das principais operações . . . . . . . . . . . . . . . . 83

5.3 Problemas de teste . . . . . . . . . . . . . . . . . . . . . 86

5.4 Resultados dos testes numéricos . . . . . . . . . . . . . . . . 87

5.4 .1 Escolha dos pontos iniciais primal e dual . . . . . . . . . . 88

5.4.2 Comparação entre o uso da hessiana real e de sua aproximação por quocientes incrementais no produto matriz-vetor . . . . . . . . . 92

5.4.3 Resolução aproximada dos subproblemas do algoritmo de lagrangianos aumentados .......................... 94

5.5 Conclusões sobre os experimentos . . . . . . . . . . . . . . . 96

6 Conclusão 101

$\begin{array}{ll}\text { A Seqüências } & 103\end{array}$

B Teorema generalizado de Gordan $\quad 107$

$\begin{array}{ll}\text { Referências bibliográficas } & 110\end{array}$ 


\section{Notações}

$\mathbb{R}^{n}$ : espaço euclideano de dimensão $n$.

$\mathbb{R}^{m \times n}$ : espaço das matrizes reais de dimensão $m \times n$.

$\mathbb{S}^{m}$ : espaço das matrizes reais simétricas de dimensão $m \times m$.

$\mathbb{S}_{+}^{m}\left(\mathbb{S}_{-}^{m}\right):$ cone das matrizes reais semidefinidas positivas (negativas) de dimensão $m \times m$.

$\mathbb{S}_{++}^{m}\left(\mathbb{S}_{--}^{m}\right):$ cone das matrizes reais definidas positivas (negativas) de dimensão $m \times m$.

$x^{i}: \quad i$-ésima componente de um vetor real $x$

$x^{T} y$ : produto interno euclidiano associado a $\mathbb{R}^{n}$ e definido por $\langle x, y\rangle \stackrel{\text { def }}{=} x^{T} y=\sum_{i=1}^{n} x_{i} y_{i}$.

$\|x\|:$ norma euclidiana sobre $\mathbb{R}^{n}$ definida por $\|x\| \stackrel{\text { def }}{=} \sqrt{\langle x, x\rangle}$.

$A=\left(a_{i j}\right) \in \mathbb{R}^{m \times n}:$ matriz real de dimensão $m \times n$ cujo elemento na $i$-ésima linha e $j$-ésima coluna é dado por $a_{i j}$ para $i \in\{1, \ldots, m\}$ e $j \in\{1, \ldots, n\}$.

$A^{T}$ : transposta de uma matriz real.

Im: matriz identidade de dimensão $m \times m$. Quando não houver possibilidade de ambigüidade no texto, usaremos apenas $I$ para denotar tal matriz.

$(A)_{i j}$ : elemento de $A$ na $i$-ésima linha e $j$-ésima coluna.

$[A]^{i}: \quad i$-ésima coluna de uma matriz real $A$.

$[A]_{i}: \quad i$-ésima linha de uma matriz real $A$.

$p_{A}(t)$ : polinômio característico de uma matriz real $A$.

$\sigma(A)$ : espectro de uma matriz real $A$.

$\lambda_{1}(A), \ldots, \lambda_{m}(A)$ : autovalores de $A \in \mathbb{R}^{m \times m}$.

$\lambda_{\min }(A)$ : menor autovalor de uma matriz $A \in \mathbb{S}^{m}$.

$\lambda_{\max }(A)$ : maior autovalor de uma matriz $A \in \mathbb{S}^{m}$.

$\operatorname{tr}(A)$ : traço de uma matriz $A \in \mathbb{R}^{m \times m}$.

$\|A\|_{F}=\|A\|:$ norma de Frobenius sobre $\mathbb{R}^{m \times n}$ definida por $\|A\|_{F} \stackrel{\text { def }}{=} \sqrt{\langle A, A\rangle}=\operatorname{tr}\left(A^{T} A\right)$. 
$\operatorname{Diag}(x)$ : matriz diagonal real de dimensão $m \times m$ cujo $i$ elemento da diagonal principal é $x_{i}$, para $i=1, \ldots, m$.

$\operatorname{diag}(A)$ : vetor real cuja $i$-ésima componente é o $i$-ésimo elemento da diagonal de $A$, para $i=1, \ldots, m$.

$A \succeq B(A \succ B)$ : indica uma ordem parcial entre duas matrizes simétricas $A, B \in \mathbb{S}^{m}$ tal que $A-B \in \mathbb{S}_{+}^{m}\left(A-B \in \mathbb{S}_{++}^{m}\right)$. Analogamente, $A \preceq B(A \prec B)$ se e somente se $A-B \in \mathbb{S}_{-}^{m}\left(A-B \in \mathbb{S}_{--}^{m}\right)$.

$\operatorname{Im}(f)$ : imagem de uma função $f$.

$\partial f$ : subdiferencial de uma função $f$.

$\delta_{C}$ : função indicadora de um conjunto $C$. 


\section{Introdução}

Problemas de programação semidefinida (PSD) têm despertado grande interesse recentemente graças à sua grande expressividade para modelar alguns problemas importantes, como a obtenção de soluções aproximadas de problemas combinatórios difíceis, o projeto de estruturas e problemas de estabilidade de sistemas dinâmicos. Nos últimos anos, diversos pesquisadores estudaram e desenvolveram métodos de pontos interiores para resolver (PSD), geralmente a partir da extensão de resultados existentes para programação linear. Porém, o estudo da aplicação de outros métodos reconhecidos de programação não-linear, como métodos de multiplicadores e penalidades, para resolver tal problema ainda não foi bastante explorado. Este texto visa apresentar alguns resultados relacionados à resolução de problemas da classe (PSD) através de métodos de lagrangianos aumentados.

Problemas de programação convexa semidefinida são caracterizados pela função objetivo convexa e por restrições definidas por funções convexas no $\mathbb{R}^{n}$ e funções $\succeq$-convexas no cone das matrizes semidefinidas positivas (ou semidefinidas negativas), o qual é um conjunto convexo. Desta forma, é natural pensar na utilização de métodos gerais de programação convexa para resolvê-los. Dentre tais métodos, temos os baseados em penalidades e multiplicadores de Lagrange como, por exemplo, o método de multiplicadores, proposto originalmente por Hestenes[Hes69] e Powell[Pow69]. Esse método baseia-se na idéia de substituir a resolução do problema original pela resolução de uma seqüência de problemas penalizados irrestritos, tal que a seqüência de suas soluções forneça uma aproximação cada vez melhor de uma solução do problema original. Uma das vantagens desse método é o fato de, sob hipóteses razoáveis, não ser necessário aumentar o parâmetro de penalidade de forma ilimitada para obter-se uma solução do problema que desejamos resolver, o que poderia gerar problemas de instabilidade numérica. Além disso, um enfoque bastante conhecido e o qual é a base deste texto para a compreensão dos resultados obtidos pelos métodos de multiplicadores é o estudo de análise convexa, em particular de funções conjugadas, que estabelecem a conexão entre as penalidades do método de multiplicadores e as regularizações do algoritmo de ponto proximal aplicado sobre o dual do problema que estamos tentando resolver.

Neste trabalho, nosso foco principal éuma subclasse de problemas de programação semidefinida cuja única restrição é uma função afim, que denotamos por (PSDA). Estudamos relações de dualidade e as condições de otimalidade associadas a essa classe de problemas a partir da extensão de resultados análogos existentes em programação linear e não-linear. A partir desses conceitos, começamos a estudar métodos de multiplicadores para resolver (PSDA), em particular o método de multiplicadores exponenciais e alguns outros algoritmos de lagrangianos aumentados suaves.

O texto possui a seguinte estrutura: 
No Capítulo 1, abordamos alguns conceitos importantes sobre matrizes simétricas, particularmente semidefinidas positivas, que são utilizados em definições e demonstrações posteriores. Além disso, apresentamos alguns tópicos de análise convexa e de cálculo matricial.

No Capítulo 2, introduzimos formalmente a definição de problemas de programação convexa semidefinida, com ênfase para o caso particular no qual estamos interessados, (PSDA). Em seguida, abordamos relações de dualidade, estabelecendo a conexão entre o problema original e seu dual. Por fim, são estudadas condições de otimalidade associadas a esse par de problemas.

No Capítulo 3, abordamos o algoritmo proximal interior entrópico proposto por Doljansky e Teboulle [DT99] para minimizar uma função matricial no cone de matrizes semidefinidas positivas e analisamos sua convergência. A partir desse algoritmo, analisamos o método de multiplicadores exponenciais também proposto em [DT99] para resolver (PSDA) e discutimos a convergência das seqüências geradas por esse método.

No Capítulo 4, analisamos o método de lagrangianos aumentados proposto por Mosheyev e Zibulevsky [MZ00] para resolver (PSDA). Em seguida, estudamos as propriedades de convergência desse método de multiplicadores a partir de um algoritmo de ponto proximal. No final desse capítulo, abordamos a obtenção de limitantes duais e o uso de minimizadores inexatos do lagrangiano aumentado para resolver instâncias de (PSDA) nas quais a função objetivo é linear.

No Capítulo 5, apresentamos os resultados de experimentos numéricos baseados no algoritmo abordado no Capítulo 4. Descrevemos a estrutura dos programas usados nos testes, comparamos o desempenho de diferentes escolhas do ponto inicial do problema dual, de diferentes estratégias para resolver o subproblema irrestrito em cada iteração do algoritmo de lagrangianos aumentados e de alguns critérios para aceitar uma solução aproximada de tais subproblemas.

No Capítulo 6, apresentamos a conclusão deste trabalho e apontamos, brevemente, algumas linhas de desenvolvimento futuro.

Por fim, apresentamos alguns resultados técnicos em dois apêndices, com o objetivo de manter o texto mais auto-contido. No Apêndice A, apresentamos as demonstrações de resultados relacionados às sequiências usadas em algumas demonstrações do texto, particularmente, seqüências ergódicas e aquelas associadas à entropia relativa de Kullback-Leibler.

No Apêndice B, demonstramos uma extensão do Teorema generalizado de Gordan para funções $\succeq$-convexas. Tal resultado é utilizado no Capítulo 2 para provar que a folga de dualidade é zero e que o problema dual associado a (PSDA) tem solução sob a condição de qualificação de Slater. 


\section{Capítulo 1}

\section{Conceitos básicos importantes}

Neste capítulo, apresentamos alguns resultados básicos de álgebra linear para matrizes reais, em particular, matrizes simétricas semidefinidas positivas. Além disso, também abordamos alguns tópicos de análise convexa sobre um espaço euclidiano genérico. Por fim, discutimos alguns resultados de análise real matricial, enfatizando funções matriciais primárias e suas derivadas.

Supomos que o leitor deste capítulo possui um conhecimento básico de álgebra linear, álgebra linear computacional e análise real. Nosso objetivo não é fornecer uma referência completa ou abordar de forma detalhada tais assuntos, mas apresentar resultados básicos importantes usados em capítulos posteriores e que, geralmente, não são cobertos em cursos de graduação. Para mais informações sobre o conteúdo coberto neste capítulo, sugerimos o artigo de Todd[Tod01], os livros de Horn e Johnson[HJ90, HJ94] e o editado por Wolkowicz, Saigal e Vandenberghe[WSV00].

\subsection{Matrizes reais}

Neste texto, abordamos somente matrizes com elementos reais. Além do produto tradicional de matrizes, empregamos um outro tipo de produto:

Definição 1.1.1. Dadas duas matrizes $A=\left(a_{i j}\right), B=\left(b_{i j}\right) \in \mathbb{R}^{m \times n}$, definimos o produto de Hadamard entre $A$ e $B$ como a matriz

$$
A \circ B \stackrel{\text { def }}{=}\left(a_{i j} b_{i j}\right) \in \mathbb{R}^{m \times n},
$$

ou seja, o produto coordenada a coordenada entre as duas matrizes.

A partir dessa definição, podemos ver que o produto de Hadamard é comutativo.

Uma quantidade que também exerce um papel importante neste texto é o traço de uma matriz:

Definição 1.1.2. Definimos o traço de uma matriz $A=\left(a_{i j}\right) \in \mathbb{R}^{m \times m}$ por

$$
\operatorname{tr}(A)=\operatorname{tr} A \stackrel{\text { def }}{=} \sum_{i=1}^{m} a_{i i},
$$

ou seja, a soma dos elementos da diagonal de $A$. 
A partir dessa definição, podemos ver que o traço é comutativo, ou seja,

$$
\operatorname{tr}(A B)=\operatorname{tr}(B A)
$$

para quaisquer matrizes reais $A, B \in \mathbb{R}^{m \times m}$. A partir dessa propriedade, obtemos a equação

$$
\operatorname{tr}\left(S^{-1} A S\right)=\operatorname{tr}(A)
$$

para quaisquer matrizes $A, S \in \mathbb{R}^{m \times m}$ tais que $S$ é inversível.

Abordamos agora algumas propriedades usadas neste texto sobre os autovalores de uma matriz real. Primeiramente, apresentamos uma maneira de calcular o produto dos autovalores sem conhecê-los explicitamente.

Proposição 1.1.3. Dada uma matriz real $A \in \mathbb{R}^{m \times m}$, o produto de seus autovalores é dado por

$$
\operatorname{det}(A)=\prod_{i=1}^{m} \lambda_{i}(A)
$$

Caracterizamos a seguir como calcular a soma de seus autovalores a partir de seu traço.

Proposição 1.1.4 ([HJ90, Teorema 1.2.12]). Para qualquer matriz real $A \in \mathbb{R}^{m \times m}$, temos

$$
\operatorname{tr}(A)=\sum_{i=1}^{m} \lambda_{i}(A)
$$

\subsection{Matrizes simétricas}

Denotamos por $\mathbb{S}^{m}$ o conjunto de todas as matrizes reais simétricas de dimensão $m \times m$. Já que grande parte das matrizes abordadas neste texto são reais e simétricas, apresentamos agora alguns fatos relevantes sobre essas matrizes.

O primeiro teorema afirma que os autovalores de uma matriz simétrica são reais e mostra uma maneira de construir uma nova matriz simétrica a partir de uma matriz simétrica.

Teorema 1.2.1 ([HJ90, Teorema 4.1.3]). Seja $A \in \mathbb{S}^{m}$ uma matriz real simétrica. Logo,

(a) todos os autovalores de A são reais;

(b) $S^{T} A S$ é simétrica para toda matriz $S \in \mathbb{R}^{m \times m}$.

O produto interno associado ao espaço vetorial $\mathbb{S}^{m}$ é o traço do produto de duas matrizes simétricas. Para quaisquer matrizes $A=\left(a_{i j}\right), B=\left(b_{i j}\right) \in \mathbb{S}^{m}$, temos

$$
\langle A, B\rangle \stackrel{\text { def }}{=} \operatorname{tr}(A B)=\sum_{i=1}^{m}(A B)_{i i}=\sum_{i=1}^{m} \sum_{j=1}^{m} a_{i j} b_{j i}=\sum_{i=1}^{m} \sum_{j=1}^{m} a_{i j} b_{i j} .
$$


E a norma matricial definida a partir desse produto interno é a norma de Frobenius:

$$
\|A\|_{F} \stackrel{\text { def }}{=}(\langle A, A\rangle)^{1 / 2}=\left(\sum_{i=1}^{m}\left(A^{2}\right)_{i i}\right)^{1 / 2}=\left(\sum_{i=1}^{m} \sum_{j=1}^{m}\left|a_{i j}\right|^{2}\right)^{1 / 2} .
$$

Vale ressaltar que utilizamos a mesma simbologia para indicar o produto interno nos espaços $\mathbb{R}^{n}$ e $\mathbb{S}^{m}$. Acreditamos que a adoção dessa notação torna o texto mais simples e não provoca ambigüidades, devido às representações distintas adotadas para matrizes e vetores.

A partir da nossa escolha do produto interno em $\mathbb{S}^{m}$, obtemos a proposição a seguir:

Proposição 1.2.2 ([Tod01, Fato 3]). Sejam $A, B \in \mathbb{S}^{m}$ matrizes simétricas e $S \in \mathbb{R}^{m \times m}$ uma matriz não-singular. Então,

$$
\langle A, B\rangle=\left\langle S A S^{T}, S^{-T} B S^{-1}\right\rangle .
$$

Em particular, se $S$ é ortogonal, então

$$
\langle A, B\rangle=\left\langle S A S^{T}, S B S^{T}\right\rangle .
$$

O próximo resultado relaciona matrizes simétricas com os seus autovalores e autovetores.

Teorema 1.2.3 (Teorema espectral para matrizes simétricas [HJ90, Teorema 4.1.5]). Uma matriz $A \in \mathbb{R}^{m \times m}$ é simétrica se e somente se existe uma matriz ortogonal $Q \in \mathbb{R}^{m \times m} e$ uma matriz diagonal $\Lambda=\left(\lambda_{i j}\right) \in \mathbb{R}^{m \times m}$ tal que

$$
A=Q \Lambda Q^{T}
$$

$O$ i-ésimo elemento da diagonal de $\Lambda$ é um autovalor de $A$ e a i-ésima coluna de $Q$ é um autovetor de A associado a tal autovalor, ou seja,

$$
A[Q]^{i}=\lambda_{i i}[Q]^{i}
$$

para $i=1, \ldots, m$.

Já que todos os autovalores de uma matriz real simétrica são reais, nós podemos introduzir uma ordem total sobre eles. Dessa forma, para toda matriz simétrica $A \in \mathbb{S}^{m}$, vamos supor neste texto que

$$
\lambda_{1}(A) \leq \lambda_{2}(A) \leq \cdots \leq \lambda_{m}(A)
$$

e indicamos por $\lambda(A) \stackrel{\text { def }}{=}\left(\lambda_{1}(A), \ldots, \lambda_{m}(A)\right)^{T}$ o vetor cujas componentes são os autovalores de $A$ ordenados de forma crescente. Ademais, denotamos por $\lambda_{\min }(A)$ e $\lambda_{\max }(A)$ o menor e o maior autovalor de $A$, respectivamente:

$$
\begin{aligned}
& \lambda_{\min }(A) \stackrel{\text { def }}{=} \min \{\lambda \mid \lambda \in \sigma(A)\}=\lambda_{1}(A), \\
& \lambda_{\max }(A) \stackrel{\text { def }}{=} \max \{\lambda \mid \lambda \in \sigma(A)\}=\lambda_{m}(A) .
\end{aligned}
$$

O próximo teorema, provado originalmente por von Neumman, afirma que o produto interno de duas matrizes simétricas é limitado superiormente pelo produto interno de seus autovalores. 
Teorema 1.2.4 ([Lew96, Teorema 2.2]). Para quaisquer matrizes $A, B \in \mathbb{S}^{m}$,

$$
\langle A, B\rangle=\operatorname{tr}(A B) \leq \lambda(A)^{T} \lambda(B) .
$$

A inequação acima é válida como igualdade se e somente se existe uma matriz ortogonal $Q \in \mathbb{R}^{m \times m}$ tal que $Q^{T} X Q=\operatorname{Diag}(\lambda(X))$ e $Q^{T} Y Q=\operatorname{Diag}(\lambda(Y))$. Em particular, temos $\|A\|_{F} \leq\|\lambda(A)\|$.

O teorema a seguir relaciona os autovalores de duas matrizes simétricas com os autovalores da soma dessas matrizes.

Teorema 1.2.5 (Teorema de Weyl [HJ90, Teorema 4.1.5]). Sejam A,B $\in \mathbb{S}^{m}$ matrizes reais simétricas. Então, para $k=1, \ldots, m$, temos

$$
\lambda_{k}(A)+\lambda_{1}(B) \leq \lambda_{k}(A+B) \leq \lambda_{k}(A)+\lambda_{m}(B) .
$$

Por esse teorema, nós podemos concluir que $\lambda_{\max }$ é superaditiva e $\lambda_{\min }$ é subaditiva.

Corolário 1.2.6. Para quaisquer matrizes $A, B \in \mathbb{S}^{m}$,

$$
\begin{aligned}
\lambda_{\max }(A+B) & \leq \lambda_{\max }(A)+\lambda_{\max }(B), \\
\lambda_{\min }(A)+\lambda_{\min }(B) & \leq \lambda_{\min }(A+B) .
\end{aligned}
$$

\subsection{Matrizes semidefinidas positivas}

Definição 1.3.1. Uma matriz real simétrica $A \in \mathbb{S}^{m}$ é semidefinida positiva se e somente se

$$
x^{T} A x \geq 0, \quad \text { para todo } x \in \mathbb{R}^{m} .
$$

Se a desigualdade acima for estrita para todo $x \neq 0$, então $A$ é definida positiva.

Similarmente, ao invertemos o sinal na desigualdade da Definição 1.3.1, obtemos as definições de uma matriz semidefinida negativa e definida negativa. De maneira equivalente, podemos definir que $A \in \mathbb{S}^{m}$ é semidefinida negativa (definida negativa) se $-A$ é semidefinida positiva (definida positiva). Assim, qualquer resultado sobre matrizes semidefinidas positivas possui um análogo para matrizes semidefinidas negativas. Se $A$ não é semidefinida positiva nem semidefinida negativa, então dizemos que $A$ é indefinida.

Denotamos o conjunto de matrizes semidefinidas positivas e definidas positivas de dimensão $m \times m$ por $\mathbb{S}_{+}^{m}$ e $\mathbb{S}_{++}^{m}$, respectivamente. Analogamente, representamos os conjuntos de matrizes semidefinidas negativas e definidas negativas por $\mathbb{S}_{-}^{m}$ e $\mathbb{S}_{--}^{m}$, respectivamente. A partir da Definição 1.3.1, temos $\mathbb{S}_{++}^{m} \subsetneq \mathbb{S}_{+}^{m}$ porque a matriz nula é semidefinida positiva mas não é definida positiva.

O próximo teorema define de maneira equivalente matrizes semidefinidas (definidas) positivas. 
Teorema 1.3.2 ([HJ90, Teorema 7.2.1]). Uma matriz simétrica é semidefinida positiva se e somente se todos os seus autovalores são não-negativos. Além disso, essa matriz é definida positiva se e somente se seus autovalores são estritamente positivos.

Definimos uma ordem parcial em $\mathbb{S}^{m}$ por

$$
A \succeq B \quad \text { se e somente se } \quad A-B \in \mathbb{S}_{+}^{m} .
$$

E temos a relação estrita se e somente se $A-B \in \mathbb{S}_{++}^{m}$. De maneira equivalente, definimos

$$
B \preceq A \quad \text { se e somente se } \quad B-A \in \mathbb{S}_{-}^{m} .
$$

De novo, a relação estrita vale se e somente se $B-A \in \mathbb{S}_{--}^{m}$. Lembremos que tal relação, conhecida como a ordem parcial de Löwner, é uma ordem pois satisfaz as seguintes propriedades:

(a) anti-simétrica: se $A \succeq B$ e $B \succeq A$, então $A=B$;

(b) transitiva: se $A \succeq B \succeq C$, então $A \succeq C$;

(c) reflexiva: $A \succeq A$.

É natural pensarmos sob quais operações essa ordem é preservada. Particularmente, a próxima proposição afirma que a ordem parcial de Löwner entre duas matrizes simétricas é mantida se as multiplicarmos à direita por uma matriz e à esquerda pela transposta da mesma matriz.

Proposição 1.3.3 ([HJ90, Observação 7.7.2]). Sejam $A, B \in \mathbb{S}^{m} e S \in \mathbb{R}^{m \times n}$. Então,

$$
A \succeq B \quad \text { implica } \quad S^{T} A S \succeq S^{T} B S .
$$

Além disso, se $n \leq m$ e $S$ tem posto $n$, obtemos

$$
A \succ B \quad \text { implica } \quad S^{T} A S \succ S^{T} B S .
$$

Apresentamos agora algumas propriedades de matrizes semidefinidas positivas.

Proposição 1.3.4. As afirmações abaixo são válidas para uma matriz $A=\left(a_{i j}\right) \in \mathbb{S}_{+}^{m}$ :

(a) ([HJ90, Observação 7.1.2]) Todas as submatrizes principais de A são semidefinidas positivas. Além disso, se $A$ é definida positiva, então as submatrizes principais de $A$ são definidas positivas.

(b) ([HJ90, Problema 2, pág.400]) Se A é definida positiva, então A é não-singular.

(c) ([HJ90, pág.398]) Se A é definida positiva (semidefinida positiva), então os elementos de sua diagonal são estritamente positivos (não-negativos), ou seja, $a_{i i}>0\left(a_{i i} \geq 0\right)$ para $i=1, \ldots, m$. 
(d) ([HJ90, Problema 2, pág.400]) Se $a_{i i}=0$ para algum $i \in\{1, \ldots, m\}$, então $a_{i k}=a_{k i}=$ 0 , para $k=1, \ldots, m$, ou seja, a i-ésima linha e a $i$-ésima coluna de $A$ são nulas se o i-ésimo elemento de sua diagonal for nulo.

Da mesma forma que todo número real não-negativo admite uma única raiz real nãonegativa de ordem $k$, para todo inteiro $k \geq 1$, existe um resultado similar para matrizes semidefinidas positivas.

Teorema 1.3.5 ([HJ90, Teorema 7.2.6]). Sejam $A \in \mathbb{S}_{+}^{m}$ uma matriz semidefinida positiva e $k$ um inteiro estritamente positivo. Então, existe uma única matriz semidefinida positiva $B \in \mathbb{S}_{+}^{m}$ tal que $A=B^{k}$ e que chamada de raiz $k$-ésima de A. Além disso, temos:

(a) $B A=A B$ e existe um polinômio $p$ tal que $B=p(A)$;

(b) $\operatorname{posto}(B)=$ posto $(A)$ e se $A$ é definida positiva, então $B$ é definida positiva.

Para toda matriz semidefinida positiva $A$ e todo inteiro $k \geq 1$, denotamos sua raiz $k$ ésima por $A^{1 / k}$. Neste texto, a raiz mais utilizada é a quadrada, ou seja, quando $B=A^{1 / 2}$. Ademais, já que $\left(A^{-1}\right)^{1 / 2}=\left(A^{1 / 2}\right)^{-1}$, indicamos $\left(A^{-1}\right)^{1 / 2}$ por $A^{-1 / 2}$.

Exibimos agora alguns fatos relacionados ao traço de matrizes semidefinidas positivas que são usados nos próximos capítulos.

Proposição 1.3.6 (Teorema de Fejer[Tod01, Fato 13]). Seja $A \in \mathbb{S}^{m}$. Então, $A \succeq 0$ se e somente se

$$
\langle A, B\rangle=\operatorname{tr}(A B) \geq 0, \quad \text { para toda matriz } B \in \mathbb{S}_{+}^{m} .
$$

De maneira análoga, $A \preceq 0$ se e somente se

$$
\langle A, B\rangle=\operatorname{tr}(A B) \geq 0, \quad \text { para toda matriz } B \in \mathbb{S}_{-}^{m} .
$$

Corolário 1.3.7. Sejam $A \in \mathbb{S}_{+}^{m}$ e $B \in \mathbb{S}_{-}^{m}$. Então, $\langle A, B\rangle=\operatorname{tr}(A B) \leq 0$.

Proposição 1.3.8 ([Tod01, Fato 14]). Sejam $A, B \in \mathbb{S}^{m}$ tais que $A \succ 0$ e $B \succeq 0$. Então, se $B \neq 0$, é válido que $\langle A, B\rangle=\operatorname{tr}(A B)>0$.

Proposição 1.3.9 ([Tod01, Fato 15]). Sejam $A, B \in \mathbb{S}_{+}^{m}$. Então,

$$
\langle A, B\rangle=\operatorname{tr}(A B)=0 \quad \text { se e somente se } A B=0 .
$$

O próximo resultado caracteriza o produto de Hadamard de duas matrizes semidefinidas positivas.

Proposição 1.3.10 ([HJ90, Teorema 7.5.3]). Se $A, B \in \mathbb{S}_{+}^{m}$, então $A \circ B \in \mathbb{S}_{+}^{m}$. Além disso, se $A$ e $B$ são definidas positivas, então $A \circ B$ também é definida positiva. 


\subsection{Análise convexa}

O nosso objetivo nesta seção é abordar alguns conceitos de análise convexa que servirão de base para entendermos os problemas estudados. Enfocamos um espaço euclidiano genérico $\mathcal{E}$, ou seja, um espaço vetorial de dimensão finita sobre os reais com um produto interno definido. Ao adotarmos essa forma de apresentação, podemos discutir os resultados de uma maneira mais elegante e abrangente, a qual contempla tanto $\mathbb{R}^{n}$ quanto $\mathbb{S}^{m}$. As principais referências para tais resultados foram os livros de Borwein e Lewis [BL06] e de Rockafellar [Roc70].

Um conjunto $\mathcal{C} \subseteq \mathcal{E}$ é convexo se e somente se $\alpha x+(1-\alpha) y \in \mathcal{C}$, para quaisquer $x, y \in \mathcal{C}$ e $\alpha \in[0,1]$.

Para toda função $f: \mathcal{E} \rightarrow[-\infty,+\infty]$, nós chamamos o conjunto

$$
\text { epi } f \stackrel{\text { def }}{=}\{(x, \mu) \in \mathcal{E} \times \mathbb{R} \mid f(x) \leq \mu\}
$$

de epígrafe de $f$. Dizemos que $f$ é uma função convexa se sua epígrafe é um subconjunto convexo de $\mathcal{E} \times \mathbb{R}$. Por outro lado, se $-f$ é uma função convexa, então nós dizemos que $f$ é uma função côncava. Se $f(x)>-\infty$ para todo $x \in \mathcal{E}$ ou se $f(x)<+\infty$ para todo $x \in \mathcal{E}$, então $f$ é convexa se e somente se

$$
f(\alpha x+(1-\alpha) y) \leq \alpha f(x)+(1-\alpha) f(y),
$$

para quaisquer elementos $x, y \in \mathcal{E}$ e $\alpha \in[0,1]$. Se $f(x)>-\infty$ para todo $x \in \mathcal{E}$ e a desigualdade acima é estrita para todo $x, y \in \mathcal{E}$ em que $x \neq y$ e todo $\alpha \in(0,1)$ tal que $f(x)$ e $f(y)$ são finitos, então dizemos que $f$ é estritamente convexa.

Dada um função convexa $f: \mathcal{E} \rightarrow[-\infty,+\infty]$, definimos seu domínio efetivo por

$$
\operatorname{dom} f \stackrel{\text { def }}{=}\{x \in \mathcal{E} \mid f(x)<+\infty\},
$$

isto é, a projeção de epi $f$ em $\mathcal{E}$. Se $f(x)>-\infty$ para todo $x \in \mathcal{E}$ e existe $\tilde{x} \in \mathcal{E}$ tal que $f(\tilde{x})<+\infty$, ou seja, se a epígrafe de $f$ não é vazia nem contém retas verticais, então dizemos que $f$ é própria. Caso contrário, $f$ é imprópria.

O próximo resultado apresenta conjuntos convexos relacionados a uma função convexa.

Teorema 1.4.1 ([Roc70, Teorema 4.6]). Sejam $f: \mathcal{E} \rightarrow[-\infty,+\infty]$ uma função convexa $e$ $\alpha \in[-\infty,+\infty]$. Então, os conjuntos de nivel

$$
\{x \in \mathcal{E} \mid f(x)<\alpha\} \quad e \quad\{x \in \mathcal{E} \mid f(x) \leq \alpha\}
$$

são convexos.

Dizemos que uma função arbitrária $f: \mathcal{E} \rightarrow[-\infty,+\infty]$ é fechada se sua epígrafe é um conjunto fechado. Por outro lado, a função $f$ é semicontínua inferior em um ponto $x \in \mathcal{E}$ se

$$
f(x) \leq \liminf _{k \rightarrow \infty} f\left(x_{k}\right)
$$

para toda seqüência $\left\{x_{k}\right\} \subset \mathcal{E}$ que convirja para $x$. Se $f$ é semicontínua inferior em todo ponto $x \in \mathcal{E}$, então dizemos que $f$ é semicontínua inferior.

O próximo teorema mostra a equivalência entre o fato de uma função ser semicontínua inferior, ser fechada e ter os conjuntos de nível fechados. 
Teorema 1.4.2 ([Roc70, Teorema 7.1]). Seja $f: \mathcal{E} \rightarrow[-\infty,+\infty]$ uma função arbitrária. Então, as seguintes afirmações são equivalentes:

(a) $f$ é semicontínua inferior em $\mathcal{E}$;

(b) o conjunto de nível $\{x \in \mathcal{E} \mid f(x) \leq \alpha\}$ é fechado em $\mathcal{E}$ para todo $\alpha \in \mathbb{R}$;

(c) a epígrafe de $f$ é um conjunto fechado em $\mathcal{E} \times \mathbb{R}$.

Um conjunto $\mathcal{C} \subseteq \mathcal{E}$ é um cone se, para todo $x \in \mathcal{C}$ e todo real $\alpha>0$, obtemos $\alpha x \in \mathcal{C}$. Um cone convexo muito usado em otimização é o cone normal a um conjunto convexo em um ponto desse conjunto.

Definição 1.4.3. Dizemos que um vetor $d \in \mathcal{E}$ é normal a um conjunto convexo $\mathcal{C} \subseteq \mathcal{E}$ em $\bar{x} \in \mathcal{C}$ se

$$
\langle x-\bar{x}, d\rangle \leq 0 \quad \text { para todo } x \in \mathcal{C},
$$

o que equivale a

$$
\langle x, d\rangle \leq\langle\bar{x}, d\rangle \quad \text { para todo } x \in \mathcal{C} .
$$

A partir dessa desigualdade, podemos ver que o conjunto de todos os vetores $d \in \mathcal{E}$ normais a $\mathrm{C}$ em $\bar{x}$ é um cone. Chamamos tal conjunto de cone normal a $\mathrm{C}$ em $\bar{x}$ e o denotamos por $N_{\mathcal{C}}(\bar{x})$.

Pela Definição 1.3.1, vemos que $\mathbb{S}_{+}^{m}$ e $\mathbb{S}_{++}^{m}$ são cones convexos. O resultado a seguir apresenta uma descrição do cone normal a $\mathbb{S}_{+}^{m}$ em uma matriz semidefinida positiva.

Proposição 1.4.4 (baseada em [Roc70, pág.226]). Seja $\bar{A} \in \mathbb{S}_{+}^{m}$. O cone normal a $\mathbb{S}_{+}^{m}$ em $\bar{A}$ é dado por

$$
\left\{B \in \mathbb{S}_{-}^{m} \mid\langle\bar{A}, B\rangle=0\right\}
$$

Demonstração.

Seja $\mathcal{D} \stackrel{\text { def }}{=}\left\{B \in \mathbb{S}_{-}^{m} \mid\langle\bar{A}, B\rangle=0\right\}$. Provamos primeiramente que $N_{\mathbb{S}_{+}^{m}}(\bar{A}) \subseteq \mathcal{D}$. Seja $B \in N_{\mathbb{S}_{+}^{m}}(\bar{A})$, ou seja,

$$
\langle A, B\rangle \leq\langle\bar{A}, B\rangle \quad \text { para toda matriz } A \in \mathbb{S}_{+}^{m} .
$$

Pela Definição 1.3.1, sabemos que $\alpha \bar{A} \in \mathbb{S}_{+}^{m}$ para todo real $\alpha \geq 0$. Em particular, temos

$$
\langle 2 \bar{A}, B\rangle \leq\langle\bar{A}, B\rangle \quad \text { e } \quad\left\langle\frac{1}{2} \bar{A}, B\right\rangle \leq\langle\bar{A}, B\rangle,
$$

resultando que

$$
\langle\bar{A}, B\rangle \leq 0 \quad \text { e } \quad 0 \leq\langle\bar{A}, B\rangle .
$$


Ao combinarmos essas duas desigualdades, concluímos que $\langle\bar{A}, B\rangle=0$. Além disso, a partir da inequação (1.4), sabemos que

$$
\langle A, B\rangle \leq 0 \quad \text { para toda matriz } A \in \mathbb{S}_{+}^{m},
$$

resultando, pela Proposição 1.3.6, que a matriz $B$ é semidefinida negativa.

Provemos agora que $N_{\mathbb{S}_{+}^{m}}(\bar{A}) \supseteq \mathcal{D}$. Seja $B \in \mathcal{D}$, o que equivale a $B \preceq 0$ e $\langle\bar{A}, B\rangle=0$. Pelo Corolário 1.3.7, obtemos

$$
\langle A, B\rangle \leq 0=\langle\bar{A}, B\rangle \quad \text { para toda matriz } A \in \mathbb{S}_{+}^{m},
$$

implicando que $B \in N_{\mathbb{S}_{+}^{m}}(\bar{A})$, o que completa a demonstração.

Um conceito que estende a noção de gradiente para funções convexas não necessariamente diferenciáveis é o de subgradiente.

Definição 1.4.5. Seja $f: \mathcal{E} \rightarrow(-\infty,+\infty]$ um função convexa. Um vetor $y \in \mathcal{E}$ é um subgradiente de $f$ em $\bar{x} \in \mathcal{E}$ se

$$
f(x) \geq f(\bar{x})+\langle y, x-\bar{x}\rangle, \quad \text { para todo } x \in \mathcal{E} .
$$

Denominamos o conjunto de todos os subgradientes de $f$ em $\bar{x} \in \mathcal{E}$ de subdiferencial de $f$ em $\bar{x}$ e o representamos por $\partial f(\bar{x})$. De maneira análoga, quando $f$ é uma função côncava sobre $\mathcal{E}$, obtemos a definição de seu subgradiente ao invertemos a desigualdade na Definição 1.4.5. Nesse caso, também chamamos o conjunto de todos os subgradientes de $f$ em $\bar{x} \in \mathcal{E}$ de subdiferencial de $f$ em $\bar{x}$ e o representamos por $\partial f(\bar{x})^{1}$.

A partir da definição acima, obtemos a próxima proposição, que mostra a relevância de subgradientes para a resolução de problemas de otimização.

Proposição 1.4.6 ([BL06, Proposição 3.1.5]). Sejam $f: \mathcal{E} \rightarrow(-\infty,+\infty]$ uma função convexa e própria e $x^{*} \in \mathcal{E}$. Então, $x^{*}$ é um ponto de mínimo de $f$ se e somente se $0 \in \partial f\left(x^{*}\right)$, ou seja, se e somente se 0 é um subgradiente de $f$ em $x^{*}$.

O próximo teorema associa o gradiente de uma função convexa em determinado ponto com o seu subgradiente.

Teorema 1.4.7 ([Roc70, Teorema 25.1]). Sejam $f: \mathcal{E} \rightarrow(-\infty,+\infty]$ uma função convexa $e \bar{x} \in \mathcal{E}$ um ponto onde $f$ é finita. Caso $f$ seja diferenciável em $\bar{x}$, então $\nabla f(\bar{x})$ é o único subgradiente de $f$ em $\bar{x}$. Em particular,

$$
f(x) \geq f(\bar{x})+\langle\nabla f(\bar{x}), x-\bar{x}\rangle \quad \text { para todo } x \in \mathcal{E} .
$$

Por outro lado, se $f$ tem um único subgradiente em $\bar{x}$, então $f$ é diferenciável em $\bar{x}$.

Usamos a desigualdade do teorema anterior para caracterizar funções convexas diferenciáveis na próxima proposição:

\footnotetext{
${ }^{1}$ Quando $f$ é côncava, alguns autores preferem os termos supergradiente e superdiferencial ao invés de subgradiente e subdiferencial, respectivamente.
} 
Proposição 1.4.8 (Desigualdade do gradiente [Ber03, Proposição 1.2.5] e [BL06, p.38, ex.12]). Seja $f: \mathcal{E} \rightarrow \mathbb{R}$ uma função diferenciável em $\mathcal{E}$. A função $f$ é convexa em $\mathcal{E}$ se $e$ somente se

$$
f(z) \geq f(x)+\langle\nabla f(x), z-x\rangle, \text { para todos } x, z \in \mathcal{E} .
$$

Além disso, $f$ é estritamente convexa se e somente se a desigualdade acima for estrita para $x \neq z$.

Dado um conjunto $\mathcal{C} \subseteq \mathcal{E}$, definimos sua função indicadora por

$$
\delta_{\mathcal{C}}(x)= \begin{cases}0 & \text { se } x \in \mathcal{C} \\ +\infty & \text { caso contrário. }\end{cases}
$$

Podemos ver facilmente que $\mathcal{C}$ é convexo se e somente se sua função indicadora é convexa.

A seguir, caracterizamos o subdiferencial da função indicadora de um conjunto convexo não-vazio $\mathcal{C} \subseteq \mathcal{E}$ em $\bar{x} \in \mathcal{E}$. A partir da Definição 1.4 .5 , sabemos que um vetor $d \in \partial \delta_{\mathcal{C}}(\bar{x})$ se e somente se

$$
\delta_{\mathfrak{C}}(x) \geq \delta_{\mathrm{e}}(\bar{x})+\langle d, x-\bar{x}\rangle, \quad \text { para todo } x \in \mathcal{E} .
$$

A desigualdade acima equivale a $\bar{x} \in \mathcal{C}$ e $\langle d, x-\bar{x}\rangle \leq 0$ para todo $x \in \mathcal{C}$, ou seja, $d$ é normal a $\mathcal{C}$ em $\bar{x}$. Assim, se $\bar{x} \in \mathcal{C}$, então $\partial \delta_{\mathcal{C}}(\bar{x})$ é igual ao cone normal a $\mathcal{C}$ em $\bar{x}$. Se $\bar{x} \notin \mathcal{C}$, então $\partial \delta_{\mathcal{C}}(\bar{x})$ é um conjunto vazio. Particularmente, a partir dessa discussão e da Proposição 1.4.4, obtemos o resultado a seguir, o qual caracteriza o subdiferencial da função indicadora de $\mathbb{S}_{+}^{m}$ avaliada em uma matriz semidefinida positiva.

Proposição 1.4.9. Para toda matriz semidefinida positiva $A \in \mathbb{S}_{+}^{m}$, temos

$$
\partial \delta_{\mathbb{S}_{+}^{m}}(A)=N_{\mathbb{S}_{+}^{m}}(A)=\left\{B \in \mathbb{S}_{-}^{m} \mid\langle A, B\rangle=0\right\} .
$$

Para toda função $f: \mathcal{E} \rightarrow[-\infty,+\infty]$, a sua convexo conjugada é uma função $f^{*}: \mathcal{E} \rightarrow$ $[-\infty,+\infty]$ definida por

$$
f^{*}(y) \stackrel{\text { def }}{=} \sup _{x \in \mathcal{E}}\{\langle x, y\rangle-f(x)\}
$$

Essa função é convexa pois é o supremo de funções lineares. Além disso, podemos ver que

$$
\langle x, y\rangle \leq f(x)+f^{*}(y),
$$

para quaisquer $x, y \in \mathcal{E}$. Tal expressão é conhecida como a desigualdade de Fenchel. O próximo teorema apresenta condições sob as quais essa expressão é válida como uma igualdade para $f$ convexa e própria.

Teorema 1.4.10 ([Roc70, Teorema 23.5]). Sejam $f: \mathcal{E} \rightarrow(-\infty,+\infty]$ uma função convexa e própria $e x, y \in \mathcal{E}$. As seguintes afirmações são equivalentes:

(a) $y \in \partial f(x)$; 
(b) o supremo da função $h(z) \stackrel{\text { def }}{=}\langle z, y\rangle-f(z)$ em $\mathcal{E}$ é atingido em $z=x$;

(c) $f(x)+f^{*}(y)=\langle x, y\rangle$.

Além disso, se $f$ é fechada, então podemos incluir na lista acima as seguintes afirmações:

(d) $x \in \partial f^{*}(y)$;

(e) o supremo da função $\tilde{h}(u)=\langle x, u\rangle-f^{*}(u)$ é atingido em $u=y$.

Corolário 1.4.11 ([Roc70, Corolário 23.5.1]). Seja $f: \mathcal{E} \rightarrow(-\infty,+\infty]$ uma função convexa, própria e fechada. Então, $x \in \partial f^{*}(y)$ se e somente se $y \in \partial f(x)$.

\subsection{Cálculo matricial}

Nesta seção, são abordados alguns conceitos de cálculo matricial que são úteis em capítulos posteriores. Em particular, estamos interessados em funções cujo domínio seja um subconjunto do espaço de matrizes simétricas de dimensão $m \times m, \mathbb{S}^{m}$, e cujo contra-domínio seja $\mathbb{S}^{m}$ ou $\mathbb{R}=\mathbb{S}^{1}$. Também abordamos mapeamentos entre $\mathbb{R}^{n}$ e $\mathbb{S}^{m}$.

Sejam $\mathcal{E}$ e $Y$ espaços euclidianos. Dizemos que um mapa $\mathcal{A}: \mathcal{E} \rightarrow$ Y é linear se

$$
\mathcal{A}(\alpha x+\beta z)=\alpha \mathcal{A}(x)+\beta \mathcal{A}(z)
$$

para todos os pontos $x, z \in \mathcal{E}$ e todos os escalares reais $\alpha$ e $\beta$. Uma função é afim se for igual à soma de um mapa linear e uma constante.

Para todo mapa linear $\mathcal{A}: \mathcal{E} \rightarrow \mathrm{Y}$, o adjunto de $\mathcal{A}$ é um mapa linear $\mathcal{A}^{*}: \mathrm{Y} \rightarrow \mathcal{E}$ que satisfaz a propriedade

$$
\left\langle\mathcal{A}^{*}(y), x\right\rangle=\langle y, \mathcal{A}(x)\rangle
$$

para todos os pontos $x \in \mathcal{E}$ e $y \in \mathrm{Y}$. Ademais, se $\mathrm{Y}=\mathbb{R}$, então existe um único $s \in \mathcal{E}$ tal que $\mathcal{A}(x)=\langle s, x\rangle$ para todo $x \in \mathcal{E}$.

Neste texto, utilizamos principalmente um mapa linear $\mathcal{A}: \mathbb{R}^{n} \rightarrow \mathbb{S}^{m}$ definido por

$$
\mathcal{A}(x) \stackrel{\text { def }}{=} \sum_{i=1}^{n} x_{i} A_{i},
$$

em que $A_{1}, \ldots, A_{n} \in \mathbb{S}^{m}$ são matrizes reais simétricas fixadas. A partir da definição de operador adjunto, sabemos que $\langle\mathcal{A}(x), U\rangle=\left\langle x, \mathcal{A}^{*}(U)\right\rangle$, para todo $x \in \mathbb{R}^{n}$ e toda $U \in \mathbb{S}^{m}$. Logo, a partir das escolhas dos produtos internos de $\mathbb{R}^{n}$ e $\mathbb{S}^{m}$, podemos ver que $\mathcal{A}^{*}$ é o operador linear de $\mathbb{S}^{m}$ para $\mathbb{R}^{n}$ definido por

$$
\mathcal{A}^{*}(U)=\left(\begin{array}{c}
\left\langle A_{1}, U\right\rangle \\
\left\langle A_{2}, U\right\rangle \\
\vdots \\
\left\langle A_{n}, U\right\rangle
\end{array}\right), \quad \text { para toda matriz } U \in \mathbb{S}^{m} .
$$


Definimos agora funções diferenciáveis em $\mathcal{E}$. Para isso, precisamos definir primeiro a taxa de convergência de uma função quando a norma de seu argumento tende a zero.

Dizemos que uma função $g: \mathcal{E} \rightarrow \mathrm{Y}$ é $o(\|h\|)$ se para todo $\epsilon>0$, existe $\delta>0$ em que

$$
\|h\| \leq \delta \quad \text { implica } \quad\|g(h)\| \leq \epsilon\|h\| .
$$

Podemos escrever essa condição equivalentemente como

$$
\lim _{\|h\| \rightarrow 0} \frac{\|g(h)\|}{\|h\|}=0
$$

Seja $F: \mathcal{E} \rightarrow$ Y uma função arbitrária definida sobre $\mathcal{E}$. Dizemos que $F$ é diferenciável em $x \in \mathcal{E}$ se existe uma função linear $L_{x}: \mathcal{E} \rightarrow \mathrm{Y}$ tal que

$$
F(x+h)=F(x)+L_{x}(h)+o(\|h\|) .
$$

Essa condição equivale a

$$
\lim _{\|h\| \rightarrow 0} \frac{\left\|F(x+h)-F(x)-L_{x}(h)\right\|}{\|h\|}=0 .
$$

Nós denominamos o operador linear $L_{x}$ de diferencial ou derivada de $F$ em $x$, o qual denotamos por $F^{\prime}(x)$ ou $\mathrm{D} F(x)$. Particularmente, uma função $f: \mathcal{E} \rightarrow \mathbb{R}$ é diferenciável em $x \in \mathcal{E}$ se existe uma função linear $\ell_{x}: \mathcal{E} \rightarrow \mathbb{R}$ tal que

$$
f(x+h)=f(x)+\ell_{x}(h)+o(\|h\|) .
$$

Pelo que vimos há pouco, existe um único $s \in \mathcal{E}$ tal que $\ell_{x}(h)=\langle s, h\rangle$ para todo $h \in \mathcal{E}$. O vetor $s$ é chamado de gradiente de $f$ em $x$ e o denotamos por $\nabla f(x)$. Assim,

$$
\ell_{x}(h)=\langle\nabla f(x), h\rangle \quad \text { para todo } h \in \mathcal{E} .
$$

Nesse caso, para $Y=\mathbb{R}$, também denotamos $\ell_{x}$ por $\mathrm{d} f(x)$, além das notações $f^{\prime}(x)$ e $\mathrm{D} f(x)$.

Vamos calcular a derivada de um operador linear $\mathcal{A}: \mathcal{E} \rightarrow$ Y. Devido a $\mathcal{A}$ ser linear, para todo $h \in \mathcal{E}$ temos

$$
\mathcal{A}(x+h)=\mathcal{A}(x)+\mathcal{A}(h),
$$

resultando que $\mathrm{D} \mathcal{A}(x)=\mathcal{A}$.

Apresentamos agora o gradiente e o diferencial de algumas funções relevantes neste texto e, em seguida, definimos funções matriciais primárias.

Proposição 1.5.1 ([Sil06, Exemplo 2.3.1]). Seja tr: $\mathbb{S}^{m} \rightarrow \mathbb{R}$ o traço de uma matriz. O gradiente em $X \in \mathbb{S}^{m}$ e o seu diferencial em $H \in \mathbb{S}^{m}$ são dados por

$$
\nabla \operatorname{tr}(X)=I_{m} \quad e \quad d \operatorname{tr}(X)[H]=\left\langle I_{m}, H\right\rangle
$$

respectivamente. 
Demonstração. Já que o traço é um função linear, para toda matriz simétrica $H \in \mathbb{S}^{m}$, obtemos

$$
\operatorname{tr}(X+H)=\operatorname{tr}(X)+\operatorname{tr}(H)=\operatorname{tr}(X)+\left\langle I_{m}, H\right\rangle,
$$

implicando que $\nabla \operatorname{tr}(X)=I_{m}$ e $\mathrm{d} \operatorname{tr}(X)[H]=\left\langle I_{m}, H\right\rangle$.

Proposição 1.5.2 ([Sil06, Exemplo 2.3.2]). Seja $f: \mathbb{S}^{m} \rightarrow \mathbb{R}$ definida por $f(X)=\operatorname{tr}\left(X^{k}\right)$, em que $k$ é um inteiro estritamente positivo e $X=\left(x_{i j}\right) \in \mathbb{S}^{m}$. Então,

$$
\nabla f(X)=k X^{k-1} \quad \text { e } \quad d \operatorname{tr}\left(X^{k}\right)[H]=\left\langle k X^{k-1}, H\right\rangle .
$$

Demonstração. Para toda matriz simétrica $H \in \mathbb{S}^{m}$, temos

$$
\begin{aligned}
f(X+H) & =\operatorname{tr}\left((X+H)^{k}\right) \\
& =\operatorname{tr}\left(X^{k}\right)+k \operatorname{tr}\left(X^{k-1} H\right)+\sum_{i=2}^{k}\left(\begin{array}{c}
k \\
i
\end{array}\right) \operatorname{tr}\left(X^{k-i} H^{i}\right) \\
& =f(X)+k\left\langle X^{k-1}, H\right\rangle+\sum_{i=2}^{k}\left(\begin{array}{c}
k \\
i
\end{array}\right)\left\langle X^{k-i}, H^{i}\right\rangle \\
& =f(X)+\left\langle k X^{k-1}, H\right\rangle+\sum_{i=2}^{k}\left(\begin{array}{c}
k \\
i
\end{array}\right)\left\langle X^{k-i}, H^{i}\right\rangle .
\end{aligned}
$$

Nós também sabemos que

$$
\begin{aligned}
0 \leq\left|\sum_{i=2}^{k}\left(\begin{array}{c}
k \\
i
\end{array}\right)\left\langle X^{k-i}, H^{i}\right\rangle\right| & \leq \sum_{i=2}^{k}\left(\begin{array}{c}
k \\
i
\end{array}\right)\left|\left\langle X^{k-i}, H^{i}\right\rangle\right| \\
& \leq \sum_{i=2}^{k}\left(\begin{array}{c}
k \\
i
\end{array}\right)\left\|X^{k-i}\right\|\left\|H^{i}\right\| \quad \text { [desigualdade de Cauchy-Schwarz] } \\
& \leq \sum_{i=2}^{k}\left(\begin{array}{c}
k \\
i
\end{array}\right)\|X\|^{k-i}\|H\|^{i},
\end{aligned}
$$

em que a última desigualdade acima decorre da propriedade submultiplicativa de normas matriciais. Assim, para $H \neq 0$, segue que

$$
0 \leq \frac{\left|\sum_{i=2}^{k}\left(\begin{array}{c}
k \\
i
\end{array}\right)\left\langle X^{k-i}, H^{i}\right\rangle\right|}{\|H\|} \leq \sum_{i=2}^{k}\left(\begin{array}{c}
k \\
i
\end{array}\right)\|X\|^{k-i}\|H\|^{i-1} .
$$

Logo, ao tomarmos o limite para $\|H\| \rightarrow 0$ na expressão acima, temos

$$
\lim _{\|H\| \rightarrow 0} \frac{\sum_{i=2}^{k}\left(\begin{array}{c}
k \\
i
\end{array}\right)\left|\left\langle X^{k-i}, H^{i}\right\rangle\right|}{\|H\|}=0
$$

implicando, a partir da expressão 1.10, que

$$
\nabla f(X)=k X^{k-1} \quad \text { e } \quad \mathrm{d} \operatorname{tr}\left(X^{k}\right)[H]=\left\langle k X^{k-1}, H\right\rangle,
$$

encerrando a demonstração. 


\subsubsection{Funções matriciais primárias}

Nesta subseção, introduzimos funções matriciais primárias e tratamos de algumas propriedades importantes sobre elas. Além disso, destacamos alguns resultados válidos para certas funções matriciais primárias que são essenciais para a análise de alguns algoritmos nos próximos capítulos. Tais propriedades podem vistas em [HJ94, Capítulo 6] e [MZ00, Apêndices B, C e D].

Pelo Teorema 1.2.3, sabemos que toda matriz simétrica $A \in \mathbb{S}^{m}$ admite uma decomposição espectral $Q \Lambda Q^{T}$, em que $\Lambda=\left(\lambda_{i j}\right) \in \mathbb{R}^{m \times m}$ é uma matriz diagonal cujo $i$-ésimo elemento da diagonal, $\lambda_{i i}$, é um autovalor de $A$ e $Q \in \mathbb{R}^{m \times m}$ é uma matriz ortogonal cuja $i$-ésima coluna é um autovetor de $A$ associado a $\lambda_{i i}$, para $i=1, \ldots, m$. A partir desse resultado, para cada função analítica $g: \mathbb{R} \rightarrow \mathbb{R}$, nós associamos uma função matricial primária sobre $\mathbb{S}^{m}$ definida por

$$
g(A) \stackrel{\text { def }}{=} Q g(\Lambda) Q^{T}=Q\left(\begin{array}{ccc}
g\left(\lambda_{1}(A)\right) & & 0 \\
0 & \ddots & \\
0 & & g\left(\lambda_{m}(A)\right)
\end{array}\right) Q^{T} .
$$

Vale ressaltar que utilizamos o mesmo símbolo para representar uma função analítica em $\mathbb{R}$ e a função matricial associada, já que podem ser identificadas univocamente pelos seus argumentos.

Listamos no próximo teorema algumas propriedades sobre funções matriciais primárias que decorrem diretamente da equação (1.11).

Teorema 1.5.3 ([HJ90, Corolário 6.2.10]). Sejam $f, g: \mathbb{R}^{n} \rightarrow \mathbb{R}$ funções analíticas e $A \in$ $\mathbb{S}^{m}$. Temos:

(a) se $f(t)=c$, em que c é uma constante real, então $f(A)=c I$;

(b) se $f(t)=t$ então $f(A)=A$;

(c) se $h(t) \stackrel{\text { def }}{=} f(t)+g(t)$, então $h(A)=f(A)+g(A)$;

(d) se $h(t) \stackrel{\text { def }}{=} f(t) g(t)$, então $h(A)=f(A) g(A)=g(A) f(A)$;

(e) se $g(\lambda) \neq 0$ para todo $\lambda \in \sigma(A)$ e $h(t) \stackrel{\text { def }}{=} f(t) / g(t)$, então $g(A)$ é não-singular $e$ $h(A)=f(A)[g(A)]^{-1}=[g(A)]^{-1} f(A)$.

O traço de uma função matricial primária desempenhará um papel importante neste texto. Por tal motivo, mostramos uma forma de calculá-lo sem que seja preciso conhecer a decomposição espectral completa da matriz, na qual somente seus autovalores são necessários. Pela expressão (1.11) e pela equação (1.1), obtemos

$$
\operatorname{tr}(g(A))=\operatorname{tr}\left(Q g(\Lambda) Q^{T}\right)=\operatorname{tr}\left(g(\Lambda) Q^{T} Q\right)=\operatorname{tr}(g(\Lambda))=\sum_{i=1}^{m} g\left(\lambda_{i}(A)\right) .
$$

Estendendo o resultado acima, indicamos uma maneira de calcular o traço da função $x \mapsto x g(x)$, para $x \in \mathbb{R}$, em que $g: \mathbb{R} \rightarrow \mathbb{R}$ é uma função analítica. 
Proposição 1.5.4. Sejam $g: \mathbb{R} \rightarrow \mathbb{R}$ uma função analítica, $h: \mathbb{R} \rightarrow \mathbb{R}$ definida por $h(x) \stackrel{\text { def }}{=}$ $x g(x)$ e $\Omega: \mathbb{S}^{m} \rightarrow \mathbb{R}$ dada por $\Omega(X) \stackrel{\text { def }}{=} \operatorname{tr} h(X)$. Então,

$$
\Omega(X)=\operatorname{tr}(X g(X))=\langle X, g(X)\rangle .
$$

Além disso,

$$
\Omega(X)=\sum_{i=1}^{m} \lambda_{i}(X) g\left(\lambda_{i}(X)\right)
$$

Demonstração. Pelo Teorema 1.5.3, sabemos que $h(X)=X g(X)$, implicando que

$$
\Omega(X)=\operatorname{tr}(X g(X))=\langle X, g(X)\rangle .
$$

Provemos agora a segunda parte da proposição. Seja $Q \Lambda Q^{T}$ uma decomposição espectral de $X$. A partir da definição de função matricial primária apresentada na equação (1.11), temos $g(X)=Q g(\Lambda) Q^{T}$, resultando que

$$
X g(X)=Q \Lambda Q^{T} Q g(\Lambda) Q^{T}=Q \Lambda g(\Lambda) Q^{T}
$$

Já que $\Lambda$ e $g(\Lambda)$ são matrizes diagonais, obtemos $(\Lambda g(\Lambda))_{i i}=(\Lambda)_{i i}(g(\Lambda))_{i i}=\lambda_{i}(X) g\left(\lambda_{i}(X)\right)$, para $i=1, \ldots, m$. Assim,

$$
\Omega(X)=\operatorname{tr}(X g(X))=\sum_{i=1}^{m}(\Lambda g(\Lambda))_{i i}=\sum_{i=1}^{m} \lambda_{i}(X) g\left(\lambda_{i}(X)\right),
$$

o que completa a prova.

A partir de agora, abordamos as derivadas do traço de uma função matricial primária. A próxima proposição mostra o gradiente dessa função.

Proposição 1.5.5 ([MZ00, Apêndice B] $)$. Sejam $\phi: \mathbb{R} \rightarrow \mathbb{R}$ uma função analítica e $\Phi(A) \stackrel{\text { def }}{=}$ $\operatorname{tr} \phi(A)$ o traço da função matricial primária associada a $\phi$ para toda matriz $A \in \mathbb{S}^{m}$. Então,

$$
\nabla \Phi(A)=\phi^{\prime}(A)
$$

Demonstração. Já que $\phi$ é analítica, ela pode ser expandida em uma série de potências

$$
\phi(t)=\sum_{i=0}^{\infty} c_{i} t^{i}
$$

implicando que

$$
\phi^{\prime}(t)=\sum_{i=1}^{\infty} i c_{i} t^{i-1}
$$


Pelo definção de $\Phi$, temos também que

$$
\Phi(A)=\operatorname{tr} \phi(A)=\operatorname{tr}\left(\sum_{i=1}^{\infty} c_{i} A^{i}\right) .
$$

Então, para $H \in \mathbb{S}^{m}$ de norma suficientemente pequena, segue-se que

$$
\begin{aligned}
\Phi(A+H)-\Phi(A) & =\operatorname{tr}\left(\sum_{i=0}^{\infty} c_{i}(A+H)^{i}\right)-\operatorname{tr}\left(\sum_{i=0}^{\infty} c_{i} A^{i}\right) \\
& =\operatorname{tr}\left(\sum_{i=0}^{\infty} c_{i} \sum_{j=0}^{i}\left(\begin{array}{l}
i \\
j
\end{array}\right) A^{i-j} H^{j}\right)-\operatorname{tr}\left(\sum_{i=0}^{\infty} c_{i} A^{i}\right) \quad \text { [equação (1.1)] } \\
& =\operatorname{tr}\left(\sum_{i=0}^{\infty} c_{i} A^{i}\right)+\operatorname{tr}\left(\sum_{i=0}^{\infty} i c_{i} A^{i-1} H\right)+\operatorname{tr}\left(\sum_{i=0}^{\infty} c_{i} \sum_{j=2}^{i}\left(\begin{array}{l}
i \\
j
\end{array}\right) A^{i-j} H^{j}\right) \\
& -\operatorname{tr}\left(\sum_{i=0}^{\infty} c_{i} A^{i}\right) \\
& =\operatorname{tr}\left(\sum_{i=0}^{\infty} i c_{i} A^{i-1} H\right)+\operatorname{tr}\left(\sum_{i=0}^{\infty} c_{i} \sum_{j=2}^{i}\left(\begin{array}{l}
i \\
j
\end{array}\right) A^{i-j} H^{j}\right) \\
& =\operatorname{tr}\left(\sum_{i=1}^{\infty} i c_{i} A^{i-1} H\right)+o(\|H\|) \\
& =\operatorname{tr}\left(\left(\sum_{i=1}^{\infty} i c_{i} A^{i-1}\right) H\right)+o(\|H\|) \\
& =\left\langle\phi^{\prime}(A), H\right\rangle+o(\|H\|),
\end{aligned}
$$

resultando que

$$
\Phi(A+H)-\Phi(A)=\left\langle\phi^{\prime}(A), H\right\rangle+o(\|H\|) .
$$

A partir da definição de gradiente, sabemos que

$$
\Phi(A+H)-\Phi(A)=\langle\nabla \Phi(A), H\rangle+o(\|H\|) .
$$

Logo, ao compararmos as equações (1.13) e (1.14), vemos que

$$
\nabla \Phi(A)=\phi^{\prime}(A)
$$

Corolário 1.5.6 ([MZ00, Apêndice B]). Sejam $\mathcal{A}$ o mapa linear definido pela equação (1.6) e $\Phi: \mathbb{S}^{m} \rightarrow \mathbb{R}$ definida por $\Phi(A) \stackrel{\text { def }}{=} \operatorname{tr} \phi(A)$, tal que $\phi: \mathbb{R} \rightarrow \mathbb{R}$ é uma função analítica. Então,

$$
\nabla_{x} \Phi(\mathcal{A}(x))=\mathcal{A}^{*}\left(\phi^{\prime}(\mathcal{A}(x))\right)
$$


Demonstração. Seja $\Theta(x)=\Phi(\mathcal{A}(x))$, para todo $x \in \mathbb{R}^{n}$. A partir da regra da cadeia para funções diferenciáveis [Bar76, Teorema40.2], sabemos que

$$
\mathrm{D} \Theta(x)=\mathrm{D} \Phi(\mathcal{A}(x)) \circ \mathrm{D} \mathcal{A}
$$

resultando, para todo $h \in \mathbb{R}^{n}$, que

$$
\begin{aligned}
\mathrm{D} \Theta(x)[h] & =\mathrm{D} \Phi(\mathcal{A}(x))[\mathrm{D} \mathcal{A}(x)[h]] & & \\
& =\langle\nabla \Phi(\mathcal{A}(x)), \mathrm{D} \mathcal{A}(x)[h]\rangle & & \text { [equação (1.8)] } \\
& =\langle\nabla \Phi(\mathcal{A}(x)), \mathcal{A}(h)\rangle & & \text { [equação (1.9)] } \\
& =\left\langle\phi^{\prime}(\mathcal{A}(x)), \mathcal{A}(h)\right\rangle & & {[\text { Proposição 1.5.5] }} \\
& =\left\langle\mathcal{A}^{*}\left(\phi^{\prime}(\mathcal{A}(x))\right), h\right\rangle & &
\end{aligned}
$$

Logo, $\nabla \Theta(x)=\mathcal{A}^{*}\left(\phi^{\prime}(\mathcal{A}(x))\right)$, o que completa a prova.

Vale ressaltar que as Proposições 1.5.1 e 1.5.2 são casos particulares da Proposição 1.5.5. Calculamos agora a segunda derivada do traço de uma função matricial.

Proposição 1.5.7 ([MZ00, Apêndice C, Teorema 4]). Sejam $\phi: \mathbb{R} \rightarrow \mathbb{R}$ uma função analítica, $\Phi(A) \stackrel{\text { def }}{=} \operatorname{tr} \phi(A)$ o traço da função matricial primária associada a $\phi$ e $\Psi(A) \stackrel{\text { def }}{=}$ $\nabla \Phi(A)$, para toda $A \in \mathbb{S}^{m}$. Também suponhamos que $Q \Lambda Q^{T}$ seja uma decomposição espectral de A. Então,

$$
D \Psi(A)[H]=Q(S \circ \tilde{H}) Q^{T}
$$

em que $\tilde{H} \stackrel{\text { def }}{=} Q^{T} H Q$ e $S=\left(s_{i j}\right) \in \mathbb{S}^{m}$ é definida por

$$
s_{i j}= \begin{cases}\frac{\phi^{\prime}\left(\lambda_{i}(A)\right)-\phi^{\prime}\left(\lambda_{j}(A)\right)}{\lambda_{i}(A)-\lambda_{j}(A)} & \text { se } \lambda_{i}(A) \neq \lambda_{j}(A), \\ \phi^{\prime \prime}\left(\lambda_{i}(A)\right) & \text { caso contrário, }\end{cases}
$$

para $i, j=1, \ldots, m$.

Demonstração. Para simplificar a notação na prova, denotemos por $\lambda_{i}$ o $i$-ésimo maior autovalor de $A$, ou seja, $\lambda_{i}=\lambda_{i}(A)$ para $i=1, \ldots, m$. Sabemos, pela Proposição 1.5.5, que $\Psi(A)=\phi^{\prime}(A)$. Ademais, já que $\phi$ é uma função analítica, sua derivada, $\phi^{\prime}$, também é analítica. Então, suponhamos que $\phi^{\prime}$ possua a seguinte expansão em uma série de potências:

$$
\phi^{\prime}(t)=\sum_{i=0}^{\infty} \alpha_{i} t^{i}
$$

o que resulta em

$$
\Psi(A)=\phi^{\prime}(A)=\sum_{i=0}^{\infty} \alpha_{i} A^{i}
$$


Assim, para toda matriz $H \in \mathbb{S}^{m}$, obtemos

$$
\begin{aligned}
\Psi(A+H)-\Psi(A) & =\sum_{i=0}^{\infty} \alpha_{i}(A+H)^{i}-\sum_{i=0}^{\infty} \alpha_{i} A^{i} \\
& =\sum_{i=1}^{\infty} \alpha_{i}\left((A+H)^{i}-A^{i}\right) .
\end{aligned}
$$

Para todo inteiro $i>0$ e toda matriz $H \in \mathbb{S}^{m}$ de norma suficientemente pequena, temos

$$
\begin{aligned}
(A+H)^{i}-A^{i} & =\sum_{k=1}^{i} A^{i-k} H A^{k-1}+o(\|H\|) \\
& =\sum_{k=1}^{i}\left(Q \Lambda Q^{T}\right)^{i-k} H\left(Q \Lambda Q^{T}\right)^{k-1}+o(\|H\|) \\
& =\sum_{k=1}^{i} Q \Lambda^{i-k} Q^{T} H Q \Lambda^{k-1} Q^{T}+o(\|H\|) \\
& =Q\left(\sum_{k=1}^{i} \Lambda^{i-k} \tilde{H} \Lambda^{k-1}\right) Q^{T}+o(\|H\|) \\
& =Q G_{i} Q^{T}+o(\|H\|),
\end{aligned}
$$

em que $G_{i}=\left(g_{r s}^{i}\right) \in \mathbb{S}^{m}$ é uma matriz cujos elementos são definidos por

$$
g_{r s}^{i}=(\tilde{H})_{r s} \sum_{k=1}^{i} \lambda_{r}^{i-k} \lambda_{s}^{k-1}
$$

para $r, s=1, \ldots, m$. Além disso, se $\lambda_{r} \neq \lambda_{s}$, então

$$
\begin{aligned}
\sum_{k=1}^{i} \lambda_{r}^{i-k} \lambda_{s}^{k-1} & =\sum_{k=1}^{i} \frac{\lambda_{r}^{i}}{\lambda_{r}^{k}} \frac{\lambda_{s}^{k}}{\lambda_{s}}=\frac{\lambda_{r}^{i}}{\lambda_{s}} \sum_{k=1}^{i}\left(\frac{\lambda_{s}}{\lambda_{r}}\right)^{k}=\frac{\lambda_{r}^{i}}{\lambda_{s}} \frac{\lambda_{s}}{\lambda_{r}}\left(\frac{\left(\frac{\lambda_{s}}{\lambda_{r}}\right)^{i}-1}{\frac{\lambda_{s}}{\lambda_{r}}-1}\right) \\
& =\lambda_{r}^{i-1}\left(\frac{\frac{\lambda_{s}^{i}-\lambda_{r}^{i}}{\lambda_{r}^{i}}}{\frac{\lambda_{s}-\lambda_{r}}{\lambda_{r}}}\right)=\frac{\lambda_{s}^{i}-\lambda_{r}^{i}}{\lambda_{s}-\lambda_{r}}=\frac{\lambda_{r}^{i}-\lambda_{s}^{i}}{\lambda_{r}-\lambda_{s}}
\end{aligned}
$$

Por outro lado, se $\lambda_{r}=\lambda_{s}$, obtemos

$$
\sum_{k=1}^{i} \lambda_{r}^{i-k} \lambda_{s}^{k-1}=\sum_{k=1}^{i} \lambda_{r}^{i-1}=i \lambda_{r}^{i-1} .
$$

Assim,

$$
\sum_{k=1}^{i} \lambda_{r}^{i-k} \lambda_{s}^{k-1}= \begin{cases}\frac{\lambda_{r}^{i}-\lambda_{s}^{i}}{\lambda_{r}-\lambda_{s}} & \text { se } \lambda_{r} \neq \lambda_{s} \\ i \lambda_{r}^{i-1} & \text { caso contrário. }\end{cases}
$$


Ao substituirmos a expressão (1.16) na equação (1.15), obtemos

$$
\begin{aligned}
\Psi(A+H)-\Psi(A) & =\sum_{i=1}^{\infty} \alpha_{i} Q G_{i} Q^{T}+o(\|H\|) \\
& =Q\left(\sum_{i=1}^{\infty} \alpha_{i} G_{i}\right) Q^{T}+o(\|H\|) .
\end{aligned}
$$

Seja $\tilde{G}=\left(\tilde{g}_{r s}\right) \stackrel{\text { def }}{=} \sum_{i=1}^{\infty} \alpha_{i} G_{i}$. A partir da equação $(1.17)$, podemos ver que

$$
\tilde{g}_{r s}=\sum_{i=1}^{\infty} \alpha_{i}(\tilde{H})_{r s} \sum_{k=1}^{i} \lambda_{r}^{i-k} \lambda_{s}^{k-1},
$$

para $r, s=1, \ldots, m$. Ao substituirmos a expressão (1.18) nessa equação, temos

$$
\tilde{g}_{r s}= \begin{cases}\sum_{i=1}^{\infty} \alpha_{i} \frac{\lambda_{r}^{i}-\lambda_{s}^{i}}{\lambda_{r}-\lambda_{s}}(\tilde{H})_{r s}=\frac{\phi^{\prime}\left(\lambda_{r}\right)-\phi^{\prime}\left(\lambda_{s}\right)}{\lambda_{r}-\lambda_{s}}(\tilde{H})_{r s} & \text { se } \lambda_{r} \neq \lambda_{s}, \\ \sum_{i=1}^{\infty} \alpha_{i} i \lambda_{r}^{i-1}(\tilde{H})_{r s}=\phi^{\prime \prime}\left(\lambda_{r}\right)(\tilde{H})_{r s} & \text { caso contrário. }\end{cases}
$$

Assim,

$$
\Psi(A+H)-\Psi(A)=Q \tilde{G} Q^{T}+o(\|H\|)=Q(S \circ \tilde{H}) Q^{T}+o(\|H\|)
$$

resultando que $\mathrm{D} \Phi(A)[H]=Q(S \circ \tilde{H}) Q^{T}$, o que conclui a demonstração.

Corolário 1.5.8 ([MZ00, Apêndice C, Corolário 1]). Sejam $\phi: \mathbb{R} \rightarrow \mathbb{R}$ uma função analítica, $\Phi(A) \stackrel{\text { def }}{=} \operatorname{tr} \phi(A)$ o traço da função matricial primária associada a $\phi$ e $\mathcal{A}: \mathbb{R}^{n} \rightarrow \mathbb{S}^{m}$ o operador linear definido pela equação (1.6). Então, a i-ésima coluna da matriz hessiana $\nabla_{x x}^{2} \Phi(\mathcal{A}(x))$ é dada por

$$
\left[\nabla_{x x}^{2} \Phi\right]^{i}=\mathcal{A}^{*}\left(Q\left(S \circ\left(Q^{T} A_{i} Q\right)\right) Q^{T}\right)
$$

em que $Q \Lambda Q^{T}$ é uma decomposição espectral de $\mathcal{A}(x)$ e $S$ é a matriz definida pela Proposição 1.5 .7 para $\mathcal{A}(x)$.

Tratamos agora de alguns resultados de análise convexa aplicados a funções matriciais primárias e ao traço dessas funções. O próximo lema mostra que a convexidade de uma função analítica implica na convexidade do traço da função matricial primária associada a ela.

Lema 1.5.9. Se $\phi: \mathbb{R} \rightarrow \mathbb{R}$ é uma função convexa e analítica, então o traço da função matricial primária associada a $\phi$ e definida sobre $\mathbb{S}^{m}, \Phi(A) \stackrel{\text { def }}{=} \operatorname{tr} \phi(A)$, também é uma função convexa. 
Demonstração. Sejam $A, X \in \mathbb{S}^{m}$. Pela Proposição 1.5.5, sabemos que $\Phi$ é diferenciável e que

$$
\begin{aligned}
& \Phi(A)+\langle X-A, \nabla \Phi(A)\rangle=\Phi(A)+\left\langle X-A, \phi^{\prime}(A)\right\rangle \\
& =\Phi(A)+\left\langle X, \phi^{\prime}(A)\right\rangle-\left\langle A, \phi^{\prime}(A)\right\rangle \\
& \leq \Phi(A)+\lambda(X)^{T} \lambda\left(\phi^{\prime}(A)\right)-\left\langle A, \phi^{\prime}(A)\right\rangle \\
& =\sum_{i=1}^{m} \phi\left(\lambda_{i}(A)\right)+\lambda(X)^{T} \lambda\left(\phi^{\prime}(A)\right)-\operatorname{tr}\left(A \phi^{\prime}(A)\right) \quad \text { [equação (1.12)] } \\
& =\sum_{i=1}^{m} \phi\left(\lambda_{i}(A)\right)+\lambda(X)^{T} \lambda\left(\phi^{\prime}(A)\right) \\
& -\sum_{i=1}^{m} \lambda_{i}(A) \phi^{\prime}\left(\lambda_{i}(A)\right) \\
& =\sum_{i=1}^{m} \phi\left(\lambda_{i}(A)\right)+\sum_{i=1}^{m}\left(\lambda_{i}(X)-\lambda_{i}(A)\right) \phi^{\prime}\left(\lambda_{i}(A)\right) \\
& =\sum_{i=1}^{m}\left(\phi\left(\lambda_{i}(A)\right)+\left(\lambda_{i}(X)-\lambda_{i}(A)\right) \phi^{\prime}\left(\lambda_{i}(A)\right)\right) \text {, }
\end{aligned}
$$

implicando que

$$
\Phi(A)+\langle X-A, \nabla \Phi(A)\rangle \leq \sum_{i=1}^{m}\left(\phi\left(\lambda_{i}(A)\right)+\left(\lambda_{i}(X)-\lambda_{i}(A)\right) \phi^{\prime}\left(\lambda_{i}(A)\right)\right) .
$$

Por hipótese, sabemos que $\phi$ é uma função convexa. Logo, para $i=1, \ldots, m$, sabemos pela desigualdade do gradiente (Proposição 1.4.8) que

$$
\phi\left(\lambda_{i}(A)\right)+\left(\lambda_{i}(X)-\lambda_{i}(A)\right) \phi^{\prime}\left(\lambda_{i}(A)\right) \leq \phi\left(\lambda_{i}(X)\right) .
$$

Ao somarmos a desigualdade acima para todo $i \in\{1, \ldots, m\}$, temos

$$
\sum_{i=1}^{m}\left(\phi\left(\lambda_{i}(A)\right)+\left(\lambda_{i}(X)-\lambda_{i}(A)\right) \phi^{\prime}\left(\lambda_{i}(A)\right)\right) \leq \sum_{i=1}^{m} \phi\left(\lambda_{i}(X)\right)=\Phi(X),
$$

em que a igualdade na expressão acima decorre da Proposição 1.5.4. Ao combinarmos as desigualdades (1.19) e (1.20), segue que

$$
\Phi(A)+\langle X-A, \nabla \Phi(A)\rangle \leq \Phi(X),
$$

o que resulta, a partir da desigualdade do gradiente novamente (Proposição 1.4.8), que a função $\Phi$ é convexa.

O próximo lema determina uma maneira de calcular a convexo conjugada de uma função definida em $\mathbb{R}^{n}$ como a soma de uma função escalar aplicada sobre cada coordenada dos elementos desse espaço. Tal função pode ser vista como uma generalização do traço de uma função matricial primária. 
Lema 1.5.10. Dada uma função $f: \mathbb{R} \rightarrow \mathbb{R}$, definimos $F: \mathbb{R}^{n} \rightarrow \mathbb{R}$ por $F(x) \stackrel{\text { def }}{=} \sum_{i=1}^{n} f\left(x_{i}\right)$. Então,

$$
F^{*}(y)=\sum_{i=1}^{n} f^{*}\left(y_{i}\right) .
$$

Demonstração. Para todo $v \in \mathbb{R}$, sabemos que

$$
f^{*}(v)=\sup _{t \in \mathbb{R}}\{\langle t, v\rangle-f(t)\} .
$$

Além disso, para todo $y \in \mathbb{R}^{n}$,

$$
\begin{aligned}
F^{*}(y) & =\sup _{x \in \mathbb{R}^{n}}\{\langle x, y\rangle-F(x)\} \\
& =\sup _{x \in \mathbb{R}^{n}}\left\{\sum_{i=1}^{n} x_{i} y_{i}-\sum_{i=1}^{n} f\left(x_{i}\right)\right\} \\
& =\sup _{x \in \mathbb{R}^{n}}\left\{\sum_{i=1}^{n}\left(x_{i} y_{i}-f\left(x_{i}\right)\right)\right\} \\
& =\sum_{i=1}^{n} \sup _{x_{i} \in \mathbb{R}}\left\{x_{i} y_{i}-f\left(x_{i}\right)\right\} \\
& =\sum_{i=1}^{n} f^{*}\left(y_{i}\right),
\end{aligned}
$$

resultando que $F^{*}(y)=\sum_{i=1}^{n} f^{*}\left(y_{i}\right)$.

O Teorema 1.5.11 relaciona a convexo conjugada de uma função matricial associada a uma função analítica com a convexo conjugada do traço dessa função matricial.

Teorema 1.5.11 ([See97, Teorema 2.1]). Sejam $\phi: \mathbb{R} \rightarrow \mathbb{R}$ uma função analítica e $\Phi(A) \stackrel{\text { def }}{=}$ $\operatorname{tr}(\phi(A))$ o traço da função matricial primária associada a $\phi$ e definida sobre $\mathbb{S}^{m}$. Então,

$$
\Phi^{*}(B)=\operatorname{tr}\left(\phi^{*}(B)\right)
$$

Demonstração. Seja $B \in \mathbb{S}^{m}$ uma matriz simétrica com decomposição espectral $Q \Lambda Q^{T}$.

Sabemos que

$$
\begin{aligned}
\Phi^{*}(B) & =\sup _{A \in \mathbb{S}^{m}}\{\langle A, B\rangle-\Phi(A)\} \\
& =\sup _{A \in \mathbb{S}^{m}}\left\{\left\langle A, Q \Lambda Q^{T}\right\rangle-\Phi(A)\right\} \\
& =\sup _{A \in \mathbb{S}^{m}}\left\{\left\langle Q^{T} A Q, \Lambda\right\rangle-\Phi(A)\right\} \quad \text { [Proposição 1.2.2]. }
\end{aligned}
$$

A função $\mathcal{H}: \mathbb{S}^{m} \rightarrow \mathbb{S}^{m}$ definida por $\mathcal{H}(A) \stackrel{\text { def }}{=} Q^{T} A Q$, para toda matriz $A \in \mathbb{S}^{m}$, introduz um isomorfismo de $\mathbb{S}^{m}$ para $\mathbb{S}^{m}$. Além disso, a partir da equação (1.12), temos $\Phi(A)=$ 
$\Phi\left(Q^{T} A Q\right)$. Assim,

$$
\begin{aligned}
\sup _{A \in \mathbb{S}^{m}}\left\{\left\langle Q^{T} A Q, \Lambda\right\rangle-\Phi(A)\right\} & =\sup _{A \in \mathbb{S}^{m}}\left\{\left\langle Q^{T} A Q, \Lambda\right\rangle-\Phi\left(Q^{T} A Q\right)\right\} \\
& =\sup _{A \in \mathbb{S}^{m}}\{\langle A, \Lambda\rangle-\Phi(A)\},
\end{aligned}
$$

implicando, a partir das expressões (1.21) e (1.22), que

$$
\Phi^{*}(B)=\sup _{A \in \mathbb{S}^{m}}\{\langle A, \Lambda\rangle-\Phi(A)\}
$$

Seja $f: \mathbb{R}^{m} \rightarrow \mathbb{R}$ definida por $f(x) \stackrel{\text { def }}{=} \sum_{i=1}^{m} \phi\left(x_{i}\right)$. A partir da decomposição espectral de $B$, sabemos também que $\Lambda=\operatorname{Diag}(\lambda(B))$. Logo,

$$
\begin{aligned}
\Phi^{*}(B) & =\sup _{A \in \mathbb{S}^{m}}\{\langle A, \operatorname{Diag}(\lambda(B))\rangle-f(\lambda(A))\} \\
& =\sup _{x \in \mathbb{R}^{m}}\left\{\left\langle U \operatorname{Diag}(x) U^{T}, \operatorname{Diag}(\lambda(B))\right\rangle-f(x) \mid U \in \mathbb{S}^{m} \text { é ortogonal }\right\} \\
& \geq \sup _{x \in \mathbb{R}^{m}}\left\{\left\langle I \operatorname{Diag}(x) I^{T}, \operatorname{Diag}(\lambda(B))\right\rangle-f(x)\right\} \\
& =\sup _{x \in \mathbb{R}^{m}}\{\langle x, \lambda(B)\rangle-f(x)\} \\
& =f^{*}(\lambda(B))
\end{aligned}
$$

resultando que

$$
\Phi^{*}(B) \geq f^{*}(\lambda(B))
$$

Provemos agora que $f^{*}(\lambda(B)) \geq \Phi^{*}(B)$. Para toda matriz $A \in \mathbb{S}^{m}$, pela desigualdade de Fenchel (inequação (1.5)) e pelo Teorema 1.2.4, temos

$$
f(\lambda(A))+f^{*}(\lambda(B)) \geq\langle\lambda(A), \lambda(B)\rangle
$$

e

$$
\langle\lambda(A), \lambda(B)\rangle \geq\langle A, B\rangle
$$

respectivamente, implicando que

$$
f(\lambda(A))+f^{*}(\lambda(B))=\Phi(A)+f^{*}(\lambda(B)) \geq\langle A, B\rangle .
$$

A desigualdade acima equivale a

$$
f^{*}(\lambda(B)) \geq\langle A, B\rangle-\Phi(A),
$$

para toda matriz $A \in \mathbb{S}^{m}$, o que resulta em

$$
f^{*}(\lambda(B)) \geq \sup _{A \in \mathbb{S} m}\{\langle A, B\rangle-\Phi(A)\}=\Phi^{*}(B),
$$

implicando que

$$
f^{*}(\lambda(B)) \geq \Phi^{*}(B) .
$$


A partir das desigualdades (1.23) e (1.24), obtemos $f^{*}(\lambda(B))=\Phi^{*}(B)$. E pelo Lema 1.5.10, sabemos que

$$
f^{*}(\lambda(B))=\sum_{i=1}^{m} \phi^{*}\left(\lambda_{i}(B)\right)=\operatorname{tr}\left(\phi^{*}(B)\right) .
$$

Então, segue que $\Phi^{*}(B)=\operatorname{tr}\left(\phi^{*}(B)\right)$, o que encerra a demonstração.

Para encerrar este capítulo, apresentamos algumas propriedades da função matricial $X \log X$, a qual é bastante relevante no Capítulo 3. O corolário abaixo decorre diretamente do Teorema 1.5.5 e da Proposição 1.5.4, ao tomarmos $\phi(x)=x \ln x$.

Corolário 1.5.12 ([Sil06, Exemplo 2.4.1]). Seja $\Omega: \mathbb{S}_{++}^{m} \rightarrow \mathbb{R}$ definida por $\Omega(X)=$ $\operatorname{tr}(X \ln X)=\langle X, \ln X\rangle$. Então, a função $\Omega$ é contínua em $\mathbb{S}_{++}^{m} e$

$$
\nabla \Omega(X)=\ln X+I \text {. }
$$

Já sabemos, pelo Lema 1.5.9, que a função $\operatorname{tr}(X \ln X)$ é convexa. Provemos agora que tal função é estritamente convexa.

Teorema 1.5.13 ([Sil06, Exemplo 2.4.1]). A função $\Omega(X) \stackrel{\text { def }}{=} \operatorname{tr}(X \ln X)=\langle X, \ln X\rangle$ é estritamente convexa em $\mathbb{S}_{++}^{m}$.

Demonstração. Seja $D_{\Omega}: \mathbb{S}_{++}^{m} \times \mathbb{S}_{++}^{m} \rightarrow \mathbb{R}$ definida por

$$
D_{\Omega}(X, Y) \stackrel{\text { def }}{=} \Omega(X)-\Omega(Y)-\langle\nabla \Omega(Y), X-Y\rangle .
$$

Provar que $\Omega(\cdot)$ é uma função estritamente convexa é equivalente a provar que $D_{\Omega}(\cdot, \cdot)>0$ se os seus argumentos são diferentes.

Para quaisquer matrizes $X, Y \in \mathbb{S}_{++}^{m}$, temos

$$
\begin{aligned}
D_{\Omega}(X, Y) & =\Omega(X)-\Omega(Y)-\langle\nabla \Omega(Y), X-Y\rangle \\
& =\langle X, \ln X\rangle-\langle Y, \ln Y\rangle-\langle\ln Y+I, X-Y\rangle \\
& =\langle X, \ln X\rangle-\langle X, \ln Y\rangle-\langle I, X\rangle+\langle I, Y\rangle \\
& \geq\langle X, \ln X\rangle-\sum_{i=1}^{m} \lambda_{i}(X) \ln \lambda_{i}(Y)-\langle I, X\rangle+\langle I, Y\rangle \quad \text { [Teorema 1.2.4] } \\
& =\sum_{i=1}^{m} \lambda_{i}(X) \ln \lambda_{i}(X)-\sum_{i=1}^{m} \lambda_{i}(X) \ln \lambda_{i}(Y) \quad \text { [Proposição 1.5.4] } \\
& -\sum_{i=1} \lambda_{i}(X)+\sum_{i=1}^{m} \lambda_{i}(Y) \\
& =\sum_{i=1}^{m}\left(\lambda_{i}(X) \ln \frac{\lambda_{i}(X)}{\lambda_{i}(Y)}-\lambda_{i}(X)+\lambda_{i}(Y)\right) \\
& =\sum_{i=1}^{m} \mathrm{~d}_{\mathrm{KL}}\left(\lambda_{i}(X), \lambda_{i}(Y)\right)
\end{aligned}
$$


em que $\mathrm{d}_{\mathrm{KL}}: \mathbb{R}_{+} \times \mathbb{R}_{++} \rightarrow \mathbb{R}$ é a entropia relativa de Kullback-Leibler definida por

$$
\mathrm{d}_{\mathrm{KL}}(a, b) \stackrel{\text { def }}{=} a \log a-a \log b+b-a .
$$

Assim, para $i=1, \ldots, m$, sabemos que $\mathrm{d}_{\mathrm{KL}}\left(\lambda_{i}(X), \lambda_{i}(Y)\right) \geq 0$ e a igualdade é válida se e somente se $\lambda_{i}(X)=\lambda_{i}(Y)$. Como resultado, pela expressão (1.25), temos

$$
D_{\Omega}(X, Y) \geq \sum_{i=1}^{m} \mathrm{~d}_{\mathrm{KL}}\left(\lambda_{i}(X), \lambda_{i}(Y)\right) \geq 0 .
$$

Além disso, pelo Teorema 1.2.4, a primeira inequação acima é válida como igualdade se e somente se existe uma matriz ortogonal $Q \in \mathbb{R}^{m \times m}$ tal que $Q^{T} X Q=\operatorname{Diag}(\lambda(X))$ e $Q^{T} Y Q=\operatorname{Diag}(\lambda(Y))$. Logo, $D(X, Y)=0$ se e somente se $\lambda(X)=\lambda(Y)$ e existe uma matriz ortogonal $Q \in \mathbb{R}^{m \times m}$ tal que $Q^{T} X Q=\operatorname{Diag}(\lambda(X))$ e $Q^{T} Y Q=\operatorname{Diag}(\lambda(Y))$, o que é equivalente a $X$ e $Y$ terem o mesmo espectro e os mesmos autovetores associados, ou seja, $X=Y$. Portanto, $\Omega$ é estritamente convexa em $\mathbb{S}_{++}^{m}$. 


\section{Capítulo 2}

\section{Apresentação dos problemas}

\section{$2.1 \quad$ O problema primal}

Um problema de programação convexa semidefinida pode ser definido por

$$
\begin{aligned}
\min & f(x) \\
\text { s. a } & g_{i}(x) \leq 0, \quad i=1, \ldots, r \\
& G(x) \preceq 0 \\
& x \in \mathbb{R}^{n}
\end{aligned}
$$

em que $f, g_{i}: \mathbb{R}^{n} \rightarrow(-\infty, \infty], i=1, \ldots, r$, são funções convexas, próprias e fechadas e $G: \mathbb{R}^{n} \rightarrow \mathbb{S}^{m}$ é uma função $\succeq$-convexa, isto é,

$$
G(\alpha x+(1-\alpha) y) \preceq \alpha G(x)+(1-\alpha) G(y),
$$

para todos $x, y \in \mathbb{R}^{n}$ e todo $\alpha \in[0,1]$.

Neste texto, abordamos principalmente um problema particular de programação convexa semidefinida no qual $G$ é um mapa afim, isto é,

$$
G(x)=A_{0}+\mathcal{A}(x)=A_{0}+\sum_{i=1}^{n} x_{i} A_{i},
$$

em que $A_{i} \in \mathbb{S}^{m}, i=0, \ldots, n$ são matrizes reais simétricas fixadas, e tal restrição é a única do problema, ou seja, $r$ é igual a 0 . Denotamos esse problema por problema de programação convexa semidefinida com restrição matricial afim (PSDA) ou problema primal:

$$
\begin{array}{cl}
\min & f(x) \\
\text { s. a } & A_{0}+\mathcal{A}(x) \preceq 0, \\
& x \in \mathbb{R}^{n} .
\end{array}
$$

Supomos que a seguinte hipótese é válida para todas as instâncias de (PSDA) que estudamos nesta dissertação:

Hipótese 2.1. A função objetivo do problema primal tem valor finito ao ser avaliada no conjunto de pontos viáveis do problema, isto é,

$$
\text { se } A_{0}+\mathcal{A}(x) \preceq 0, \text { então } f(x) \in \mathbb{R} .
$$


Quando a função objetivo de (PSDA) for linear, isto é, se $f(x)=c^{T} x$, para algum vetor $c$, denotamos tal caso especial por problema de programação semidefinida linear (PSDL) ou problema primal linear:

$$
\begin{array}{cl}
\min & c^{T} x \\
\text { s. a } & A_{0}+\mathcal{A}(x) \preceq 0, \\
& x \in \mathbb{R}^{n} .
\end{array}
$$

Em algumas partes do texto, estamos interessados especificamente nesse problema, pois ele possui resultados teóricos fortes e métodos numéricos de resolução eficientes. Além disso, existem problemas reais importantes, como em otimização combinatória e no projeto de estruturas, que podem ser modelados desta forma. Veja, por exemplo, [Tod01, BTN01, WSV00]. Também é importante ressaltar que problemas de programação linear (PL) pertencem a essa categoria.

\subsection{O problema dual}

A partir de uma extensão bastante conhecida de alguns conceitos de dualidade de programação não-linear com restrições no $\mathbb{R}^{n}$ [Ber99, Capítulo 3], definimos o lagrangiano ou função lagrangiana $\mathrm{L}: \mathbb{R}^{n} \times \mathbb{S}^{m} \rightarrow \mathbb{R}$ associado a (PSDA) por

$$
\mathrm{L}(x, U) \stackrel{\text { def }}{=} f(x)+\left\langle U, A_{0}+\mathcal{A}(x)\right\rangle .
$$

Essa função é convexa em relação ao primeiro argumento e côncava em relação ao segundo.

Também de modo análogo ao feito em otimização não linear, nós apresentamos o problema lagrangiano primal associado a (PSDA):

$$
\inf _{x \in \mathbb{R}^{n}} \sup _{U \in D} \mathrm{~L}(x, U),
$$

em que o conjunto $D \subseteq \mathbb{S}^{m}$ deve ser escolhido de modo que $\sup _{U \in D} \mathrm{~L}(x, U)$ valha $f(x)$ se $x$ for um ponto viável em (PSDA) e $+\infty$ se for inviável. Neste caso, resolver (PSDA) é equivalente a resolver o problema lagrangiano primal. Ou seja, queremos que

$$
\sup _{U \in D} \mathrm{~L}(x, U)= \begin{cases}f(x) & \text { se } A_{0}+\mathcal{A}(x) \preceq 0, \\ +\infty & \text { caso contrário. }\end{cases}
$$

Assim, podemos considerar $U \in D$ como uma matriz de multas que penaliza os pontos inviáveis do problema (PSDA) e não altera o valor da função objetivo do problema primal para pontos viáveis.

Para cada $x \in \mathbb{R}^{n}$, observemos, pela Proposição 1.3.6, que $x$ é viável em (PSDA) se e somente se $\left\langle U, A_{0}+\mathcal{A}(x)\right\rangle \leq 0$ para toda matriz $U \succeq 0$. Logo, para $D=\mathbb{S}_{+}^{m}$ obtemos

$$
\sup _{U \in \mathbb{S}_{+}^{m}} \mathrm{~L}(x, U)= \begin{cases}f(x) & \text { se } A_{0}+\mathcal{A}(x) \preceq 0 \quad(x \text { é viável em PSDA }), \\ +\infty & \text { caso contrário } \quad(x \text { é inviável em PSDA })\end{cases}
$$

e concluímos que as matrizes de penalidades devem ser semidefinidas positivas. Além disso, sob a Hipótese 2.1, podemos concluir que $\sup _{U \in \mathbb{S}_{+}^{m}} \mathrm{~L}(x, U)$ é finito se $x$ é viável em (PSDA) e $+\infty$ caso contrário. 
Ao invertermos a posição do sup e do inf na definição do problema lagrangiano primal, obtemos o problema lagrangiano dual (DSDA) associado a (PSDA) ou, simplesmente, o problema dual:

$$
\sup _{U \in \mathbb{S}_{+}^{m}} \inf _{x \in \mathbb{R}^{n}} \mathrm{~L}(x, U)
$$

Seja $\mathcal{K}_{\mathrm{D}}: \mathbb{S}^{m} \rightarrow[-\infty,+\infty]$ a função objetivo do problema dual: $\mathcal{K}_{\mathrm{D}}(U) \stackrel{\text { def }}{=} \inf _{x \in \mathbb{R}^{n}} \mathrm{~L}(x, U)$. Logo, (DSDA) pode ser representado de forma mais compacta por

$$
\sup _{U \in \mathbb{S}_{+}^{m}} \mathcal{K}_{\mathrm{D}}(U)
$$

Consideremos agora apenas (PSDL). A função lagrangiana associada a esse problema é dada por

$$
\begin{aligned}
\mathrm{L}(x, U) & =c^{T} x+\left\langle U, A_{0}+\mathcal{A}(x)\right\rangle \\
& =c^{T} x+\left\langle U, A_{0}+\sum_{i=1}^{n} x_{i} A_{i}\right\rangle \\
& =c^{T} x+\left\langle U, A_{0}\right\rangle+\sum_{i=1}^{n}\left\langle U, x_{i} A_{i}\right\rangle \\
& =c^{T} x+\left\langle U, A_{0}\right\rangle+\sum_{i=1}^{n} x_{i}\left\langle U, A_{i}\right\rangle \\
& =\left\langle U, A_{0}\right\rangle+x^{T}\left(c+\mathcal{A}^{*}(U)\right) .
\end{aligned}
$$

A partir das relações acima, podemos ver que, para toda matriz $U \in \mathbb{S}^{m}$, se $c+\mathcal{A}^{*}(U) \neq 0$, então podemos escolher $\hat{x} \in \mathbb{R}^{n}$ tal que $\mathrm{L}(\hat{x}, U)$ seja tão negativo quanto se queira. Assim, para toda $U \in \mathbb{S}_{+}^{m}$, concluímos que

$$
\mathcal{K}_{\mathrm{D}}(U)=\inf _{x \in \mathbb{R}^{n}} \mathrm{~L}(x, U)= \begin{cases}\left\langle U, A_{0}\right\rangle & \text { se } c=-\mathcal{A}^{*}(U), \\ -\infty & \text { caso contrário. }\end{cases}
$$

Logo, se $\mathcal{K}_{\mathrm{D}}(\tilde{U})>-\infty$ para alguma matriz $\tilde{U} \in \mathbb{S}_{+}^{m}$, ou seja, se $c \in \operatorname{Im}\left(-\mathcal{A}^{*}\right)$, então o conjunto de soluções e o valor ótimo do problema lagrangiano dual associado a (PSDL) são iguais ao do problema dual linear (DSDL):

$$
\begin{aligned}
\max & \left\langle U, A_{0}\right\rangle \\
\text { s. a } & c+\mathcal{A}^{*}(U)=0, \\
& U \in \mathbb{S}_{+}^{m} .
\end{aligned}
$$

Esse problema não tem pontos viáveis quando o valor ótimo do problema lagrangiano dual é igual a $-\infty$. Nesse caso, para mantermos consistência, vamos adotar a convenção de que o valor ótimo de (DSDL) é $-\infty$ quando o seu conjunto viável for vazio.

Apresentamos a seguir algumas relações entre os problemas lagrangiano primal e lagrangiano dual. 


\subsubsection{Relações de dualidade}

Sejam $\mathrm{f}_{*}^{\mathrm{P}}$ e $\mathrm{f}_{*}^{\mathrm{D}}$ os valores ótimos dos problemas primal e dual, respectivamente:

$$
\begin{aligned}
& \mathrm{f}_{*}^{\mathrm{P}} \stackrel{\text { def }}{=} \inf _{x \in \mathbb{R}^{n}}\left\{f(x) \mid A_{0}+\mathcal{A}(x) \preceq 0\right\}=\inf _{x \in \mathbb{R}^{n}} \sup _{U \in \mathbb{S}_{+}^{m}} \mathrm{~L}(x, U), \\
& \mathrm{f}_{*}^{\mathrm{D}} \stackrel{\text { def }}{=} \sup _{U \in \mathbb{S}^{m}}\left\{\mathcal{K}_{\mathrm{D}}(U) \mid U \succeq 0\right\}=\sup _{U \in \mathbb{S}_{+}^{m}} \inf _{x \in \mathbb{R}^{n}} \mathrm{~L}(x, U) .
\end{aligned}
$$

Se (PSDA) não tiver nenhum ponto viável, então adotamos a convenção de que $\mathrm{f}_{*}^{\mathrm{P}}=+\infty$. Analogamente, se (DSDL) não tiver nenhum ponto viável, definimos que $\mathrm{f}_{*}^{\mathrm{D}}$ será $-\infty$.

Será provado agora um resultado que relaciona os valores da funções objetivos dos problemas primal e dual avaliadas em pontos viáveis desses problemas:

Teorema 2.2.1 (Dualidade Fraca). Seja $(\tilde{x}, \tilde{U}) \in \mathbb{R}^{n} \times \mathbb{S}^{m}$ tal que $\tilde{x}$ é viável em (PSDA) e $\tilde{U} \succeq 0$. Então,

$$
\mathcal{K}_{\mathrm{D}}(\tilde{U}) \leq f(\tilde{x})
$$

Demonstração. Já que $A_{0}+\mathcal{A}(\tilde{x}) \preceq 0$ e que $\tilde{U} \succeq 0$, nós sabemos que $\left\langle\tilde{U}, A_{0}+\mathcal{A}(x)\right\rangle \leq 0$ pelo Corolário 1.3.7. Desta forma,

$$
\inf _{x \in \mathbb{R}^{n}} \mathrm{~L}(x, \tilde{U}) \leq \mathrm{L}(\tilde{x}, \tilde{U})=f(\tilde{x})+\left\langle\tilde{U}, A_{0}+\mathcal{A}(\tilde{x})\right\rangle \leq f(\tilde{x}),
$$

resultando que $\mathcal{K}_{\mathrm{D}}(\tilde{U})=\inf _{x \in \mathbb{R}^{n}} \mathrm{~L}(x, \tilde{U}) \leq f(\tilde{x})$.

Já que o teorema acima é válido para todo par de pontos $(\tilde{x}, \tilde{U}) \in \mathbb{R}^{n} \times \mathbb{S}^{m}$ tal que $\tilde{x}$ é viável para (PSDA) e $\tilde{U} \succeq 0$, podemos ver que os dois próximos resultados claramente são válidos.

Corolário 2.2.2. O valor ótimo de (DSDA) é um limitante inferior do valor ótimo de $(P S D A)$. Em particular, o valor ótimo de (DSDL) é um limitante inferior do valor ótimo de (PSDL):

$$
\sup _{U \in \mathbb{S}_{+}^{m}}\left\{\left\langle U, A_{0}\right\rangle \mid c=-\mathcal{A}^{*}(U)\right\} \leq \inf _{x \in \mathbb{R}^{n}}\left\{c^{T} x \mid A_{0}+\mathcal{A}(x) \preceq 0\right\} .
$$

Corolário 2.2.3. Se $\mathrm{f}_{*}^{\mathrm{D}}=+\infty$, então (PSDA) não tem pontos viáveis.

Demonstração. Suponhamos que $\mathrm{f}_{*}^{\mathrm{D}}=+\infty$. Então, pelo Corolário 2.2.2, temos $\mathrm{f}_{*}^{\mathrm{P}}=+\infty$. Entretanto, pela Hipótese 2.1, sabemos que $f(\bar{x})<+\infty$, para todo $\bar{x} \in \mathbb{R}^{n}$ viável para (PSDA). Portanto, (PSDA) não tem pontos viáveis.

Chamamos de folga de dualidade a diferença entre os valores ótimos dos problemas primal e dual, a qual é representada por $\mathcal{F}$ :

$$
\mathcal{F} \stackrel{\text { def }}{=} \mathrm{f}_{*}^{\mathrm{P}}-\mathrm{f}_{*}^{\mathrm{D}}=\inf _{x \in \mathbb{R}^{n}} \sup _{U \in \mathbb{S}_{+}^{m}} \mathrm{~L}(x, U)-\sup _{U \in \mathbb{S}_{+}^{m}} \inf _{x \in \mathbb{R}^{n}} \mathrm{~L}(x, U) .
$$


Pelo Corolário 2.2.2, sabemos que a folga de dualidade é não negativa.

Consideremos o problema de programação semidefinida linear. Para esse problema, a folga de dualidade é

$$
\mathcal{F}=\inf _{x \in \mathbb{R}^{n}}\left\{c^{T} x \mid A_{0}+\mathcal{A}(x) \preceq 0\right\}-\sup _{U \in \mathbb{S}_{+}^{m}}\left\{\left\langle U, A_{0}\right\rangle \mid c+\mathcal{A}^{*}(U)=0\right\} .
$$

Então, para $\tilde{x} \in \mathbb{R}^{n}$ viável em (PSDL) e $\tilde{U} \in \mathbb{S}_{+}^{m}$ viável em (DSDL), sabemos, pelo Teorema de dualidade fraca, que

$$
\begin{aligned}
0 & \leq c^{T} \tilde{x}-\left\langle\tilde{U}, A_{0}\right\rangle=-\mathcal{A}^{*}(\tilde{U})^{T} \tilde{x}-\left\langle\tilde{U}, A_{0}\right\rangle \\
& =-\sum_{i=1}^{n}\left\langle\tilde{U}, A_{i}\right\rangle \tilde{x}_{i}-\left\langle\tilde{U}, A_{0}\right\rangle=-\left(\sum_{i=1}^{n}\left\langle\tilde{U}, \tilde{x}_{i} A_{i}\right\rangle+\left\langle\tilde{U}, A_{0}\right\rangle\right) \\
& =-\left\langle\tilde{U}, A_{0}+\sum_{i=1}^{n} \tilde{x}_{i} A_{i}\right\rangle=-\left\langle\tilde{U}, A_{0}+\mathcal{A}(\tilde{x})\right\rangle .
\end{aligned}
$$

Logo, $-\left\langle\tilde{U}, A_{0}+\mathcal{A}(\tilde{x})\right\rangle$ é igual à diferença entre os valores das funções objetivos dos problemas primal linear e dual linear avaliadas nos pontos $\tilde{x}$ e $\tilde{U}$, respectivamente. Em particular, se houver algum $x^{*} \in \mathbb{R}^{n}$ viável em (PSDL) e algum $U^{*} \in \mathbb{S}^{m}$ viável em (DSDL) tal que $\left\langle U^{*}, A_{0}+\mathcal{A}\left(x^{*}\right)\right\rangle=0$, temos então que a folga de dualidade é zero, resultando que o valor ótimo de (PSDL) é igual ao valor ótimo de (DSDL), $x^{*}$ é uma solução do problema primal e $U^{*}$ é uma solução do problema dual.

Entretanto, há situações em que a folga de dualidade é estritamente positiva. E mesmo quando tal folga é zero, há problemas para os quais não conseguimos obter uma solução para (PSDA) ou para (DSDA). O próximo exemplo ilustra uma situação em que a folga de dualidade é zero, mas não há uma solução para o problema primal.

Exemplo 2.2.1 ([Tod01, Seção 4]). Consideremos o problema

$\min x_{1}$

$$
\begin{array}{ll}
\text { s. } a & \left(\begin{array}{cc}
0 & -1 \\
-1 & 0
\end{array}\right)+x_{1}\left(\begin{array}{cc}
-1 & 0 \\
0 & 0
\end{array}\right)+x_{2}\left(\begin{array}{cc}
0 & 0 \\
0 & -1
\end{array}\right) \preceq 0, \\
& x \in \mathbb{R}^{2}
\end{array}
$$

A restrição acima é equivalente a

$$
-\left(\begin{array}{cc}
x_{1} & 1 \\
1 & x_{2}
\end{array}\right) \preceq 0
$$

A partir da decomposição em autovalores da matriz acima, é fácil ver que o conjunto viável do problema acima é $\left\{x \in \mathbb{R}_{+}^{2} \mid x_{1} x_{2} \geq 1\right\}$. Logo, podemos obter pontos viáveis para o problema acima da forma $(\epsilon, 1 / \epsilon)$, para $\epsilon$ estritamente positivo tão próximo de zero quanto se queira. Então, o valor ótimo do problema é 0 , mas não há ponto viável que atinja tal valor. 
Por outro lado, o dual desse problema é

$$
\begin{aligned}
\max & \left\langle U,\left(\begin{array}{cc}
0 & -1 \\
-1 & 0
\end{array}\right)\right\rangle \\
\text { s. a } & -\left\langle U,\left(\begin{array}{cc}
-1 & 0 \\
0 & 0
\end{array}\right)\right\rangle=1, \\
& -\left\langle U,\left(\begin{array}{cc}
0 & 0 \\
0 & -1
\end{array}\right)\right\rangle=0, \\
& U \in \mathbb{S}_{+}^{2} .
\end{aligned}
$$

A partir da análise das restrições desse problema, concluímos que o único ponto viável é

$$
U^{*}=\left(\begin{array}{ll}
1 & 0 \\
0 & 0
\end{array}\right)
$$

o qual, portanto, é uma solução do problema dual. Assim, o valor ótimo do problema dual é

$$
\left\langle\left(\begin{array}{ll}
1 & 0 \\
0 & 0
\end{array}\right),\left(\begin{array}{cc}
0 & -1 \\
-1 & 0
\end{array}\right)\right\rangle=0
$$

Logo, a folga de dualidade é 0. Porém, não há uma solução para o problema primal.

O exemplo seguinte apresenta uma instância de (PSDL) em que a folga de dualidade é estritamente positiva.

Exemplo 2.2.2 ([WSV00, Exemplo 4.1.2]).

$$
\begin{aligned}
& \min -x_{2} \\
& \text { s. a }\left(\begin{array}{ccc}
-a & 0 & 0 \\
0 & 0 & 0 \\
0 & 0 & 0
\end{array}\right)+x_{1}\left(\begin{array}{lll}
0 & 0 & 0 \\
0 & 1 & 0 \\
0 & 0 & 0
\end{array}\right)+x_{2}\left(\begin{array}{lll}
1 & 0 & 0 \\
0 & 0 & 1 \\
0 & 1 & 0
\end{array}\right) \preceq 0 \\
& \quad x \in \mathbb{R}^{2}
\end{aligned}
$$

em que $a>0$.

A restrição acima é equivalente a

$$
\left(\begin{array}{ccc}
x_{2}-a & 0 & 0 \\
0 & x_{1} & x_{2} \\
0 & x_{2} & 0
\end{array}\right) \preceq 0 .
$$

A partir da decomposição em autovalores dessa matriz, podemos ver que o conjunto viável desse problema é $\left\{x \in \mathbb{R}^{2} \mid x_{1} \leq 0\right.$ e $\left.x_{2}=0\right\}$. Logo, o valor ótimo é 0 . 
O dual desse problema é

$$
\begin{aligned}
\max & \left\langle U,\left(\begin{array}{ccc}
-a & 0 & 0 \\
0 & 0 & 0 \\
0 & 0 & 0
\end{array}\right)\right\rangle=-a u_{11} \\
\text { s. a } & -\left\langle U,\left(\begin{array}{lll}
0 & 0 & 0 \\
0 & 1 & 0 \\
0 & 0 & 0
\end{array}\right)\right\rangle=-u_{22}=0, \\
& -\left\langle U,\left(\begin{array}{lll}
1 & 0 & 0 \\
0 & 0 & 1 \\
0 & 1 & 0
\end{array}\right)\right\rangle=-u_{11}-2 u_{23}=-1, \\
& U \in \mathbb{S}_{+}^{2} .
\end{aligned}
$$

Ao analisarmos a positividade da submatriz principal de $U$

$$
\left(\begin{array}{ll}
u_{22} & u_{23} \\
u_{32} & u_{33}
\end{array}\right)=\left(\begin{array}{cc}
0 & u_{23} \\
u_{32} & u_{33}
\end{array}\right)
$$

concluímos que $u_{23}=u_{32}=0$ a partir da Proposição 1.3.4. Logo, $u_{11}=1$, resultando que o valor ótimo do problema dual é $-a$. Portanto, a folga de dualidade é $a$.

Dessa forma, o Exemplo 2.2.2 nos fornece um par de problemas primal e dual em que a folga de dualidade é tão grande quanto se queira.

Apresentamos na próxima seção condições que garantem que a folga de dualidade é zero e que, além disso, há soluções tanto para o problema primal quanto para o dual. Adicionalmente, também comentamos sobre uma condição que garante que a folga de dualidade é zero e que o conjunto de soluções do problema dual é não-vazio e compacto.

\subsection{Dualidade forte e condições de otimalidade}

Dizemos que um par $(\bar{x}, \bar{U}) \in \mathbb{R}^{n} \times \mathbb{S}^{m}$ é um ponto de sela da função lagrangiana $\mathrm{L}$ se

$$
\bar{x} \in \underset{x \in \mathbb{R}^{n}}{\operatorname{argmin}} \mathrm{L}(x, \bar{U}) \quad \text { e } \quad \bar{U} \in \underset{U \in \mathbb{S}_{+}^{m}}{\operatorname{argmax}} \mathrm{L}(\bar{x}, U)
$$

ou, equivalentemente, se $\bar{U} \in \mathbb{S}_{+}^{m}$ e

$$
\sup _{U \in \mathbb{S}_{+}^{m}} \mathrm{~L}(\bar{x}, U)=\mathrm{L}(\bar{x}, \bar{U})=\inf _{x \in \mathbb{R}^{n}} \mathrm{~L}(x, \bar{U}) .
$$

A próxima proposição relaciona um ponto de sela com soluções dos problemas primal e dual. Reescrevemos a prova existente no livro editado por Wolkowicz, Saigal e Vandenberghe [WSV00].

Proposição 2.3.1 (baseada em [WSV00, Proposição 2.1]). Sejam $\bar{x} \in \mathbb{R}^{n}$ e $\bar{U} \in \mathbb{S}^{m}$. Então, a folga de dualidade é zero, $\bar{x}$ é uma solução de (PSDA) e $\bar{U}$ é uma solução de $(D S D A)$ se e somente se $(\bar{x}, \bar{U})$ for um ponto de sela de $\mathbf{L}$. 


\section{Demonstração.}

$(\Rightarrow)$ Suponhamos que a folga de dualidade seja zero e que $\bar{x}$ e $\bar{U}$ sejam soluções de (PSDA) e de (DSDA), respectivamente.

A partir das equações (2.3) e (2.4), obtemos

$$
\begin{aligned}
& \mathrm{f}_{*}^{\mathrm{P}}=\inf _{x \in \mathbb{R}^{n}} \sup _{U \succeq 0} \mathrm{~L}(x, U)=\sup _{U \succeq 0} \mathrm{~L}(\bar{x}, U) \geq \mathrm{L}(\bar{x}, \bar{U}), \\
& \mathrm{f}_{*}^{\mathrm{D}}=\sup _{U \succeq 0} \inf _{x \in \mathbb{R}^{n}} \mathrm{~L}(x, U)=\inf _{x \in \mathbb{R}^{n}} \mathrm{~L}(x, \bar{U}) \leq \mathrm{L}(\bar{x}, \bar{U}) .
\end{aligned}
$$

Logo, como a folga de dualidade é zero,

$$
\sup _{U \succeq 0} \mathrm{~L}(\bar{x}, U)=\mathrm{L}(\bar{x}, \bar{U})=\inf _{x \in \mathbb{R}^{n}} \mathrm{~L}(x, \bar{U}) .
$$

E já que $\bar{U} \succeq 0$, nós concluímos que $(\bar{x}, \bar{U})$ é um ponto de sela de $\mathrm{L}$.

$(\Leftarrow)$ Suponhamos que $(\bar{x}, \bar{U})$ seja um ponto de sela de $\mathrm{L}$, ou seja, a matriz $\bar{U}$ é semidefinida positiva e

$$
\sup _{U \in \mathbb{S}_{+}^{m}} \mathrm{~L}(\bar{x}, U)=\mathrm{L}(\bar{x}, \bar{U})=\inf _{x \in \mathbb{R}^{n}} \mathrm{~L}(x, \bar{U}) .
$$

A partir dessa expressão, obtemos

$$
\inf _{x \in \mathbb{R}^{n}} \sup _{U \in \mathbb{S}_{+}^{m}} \mathrm{~L}(x, U) \leq \mathrm{L}(\bar{x}, \bar{U}) \leq \sup _{U \in \mathbb{S}_{+}^{m}} \inf _{x \in \mathbb{R}^{n}} \mathrm{~L}(x, U),
$$

o que equivale a $\mathrm{f}_{*}^{\mathrm{P}} \leq \mathrm{L}(\bar{x}, \bar{U}) \leq \mathrm{f}_{*}^{\mathrm{D}}$. Contudo, pelo Teorema 2.2.1, $\mathrm{f}_{*}^{\mathrm{D}} \leq \mathrm{f}_{*}^{\mathrm{P}}$. Logo, podemos concluir que a folga de dualidade é zero e que

$$
\inf _{x \in \mathbb{R}^{n}} \mathrm{~L}(x, \bar{U})=\mathrm{L}(\bar{x}, \bar{U})=\sup _{U \in \mathbb{S}_{+}^{m}} \inf _{x \in \mathbb{R}^{n}} \mathrm{~L}(x, U)=\mathrm{f}_{*}^{\mathrm{D}} .
$$

Já que $\bar{U}$ é semidefinida positiva e atinge o valor ótimo do problema dual, essa matriz é uma solução de (DSDA).

Provemos que $\bar{x}$ é uma solução do problema primal. Já que a função objetivo de (PSDA) é convexa e própria, $f(\bar{x})>-\infty$ e existe $\tilde{x} \in \operatorname{dom} f$ tal que $f(\tilde{x})$ é um número real. Além disso, o produto interno associado a $\mathbb{S}^{m}$ é sempre um número real para quaisquer matrizes simétricas. Logo,

$$
\begin{aligned}
-\infty & <f(\bar{x})+\left\langle\bar{U}, A_{0}+\mathcal{A}(\bar{x})\right\rangle \\
& =\mathrm{L}(\bar{x}, \bar{U}) \\
& =\inf _{x \in \mathbb{R}^{n}} \mathrm{~L}(x, \bar{U}) \quad[\text { expressão (2.6)] } \\
& \leq f(\tilde{x})+\left\langle\bar{U}, A_{0}+\mathcal{A}(\tilde{x})\right\rangle \in \mathbb{R},
\end{aligned}
$$

resultando que $\mathrm{L}(\bar{x}, \bar{U})$ é um número real. Como conseqüência, pela expressão (2.6), nós concluímos que $\sup _{U \in \mathbb{S}_{+}^{m}} \mathrm{~L}(\bar{x}, U)$ é um número real também. E, a partir expressão $(2.1)$, sabemos que $\bar{x}$ é uma solução de (PSDA), o que encerra a prova. 
Assim, a existência de um ponto de sela nos fornece soluções dos problemas primal e dual e, além disso, que a folga de dualidade é zero. Contudo, muitas vezes, verificar que um par de pontos é um ponto de sela não é uma tarefa fácil. Então, torna-se necessário encontrar outras condições para que um dado par de pontos seja solução de (PSDA) e de (DSDA) e que a folga de dualidade seja zero, como as que são apresentadas no teorema a seguir.

Teorema 2.3.2 (Condições KKT [Roc70, Teorema 28.3]). Sejam $x^{*} \in \mathbb{R}^{n}$ e $U^{*} \in \mathbb{S}^{m}$. Então, $x^{*}$ é uma solução do problema primal, $U^{*}$ é uma solução do problema dual e a folga de dualidade é zero se e somente se

$$
\begin{aligned}
0 \in \partial_{x} L\left(x^{*}, U^{*}\right) & & \\
A_{0}+\mathcal{A}\left(x^{*}\right) \preceq 0 & & \text { (viabilidade primal), } \\
U^{*} \succeq 0 & & \text { (viabilidade dual), } \\
\left\langle U^{*}, A_{0}+\mathcal{A}\left(x^{*}\right)\right\rangle=0 & & \text { (folgas complementares). }
\end{aligned}
$$

\section{Demonstração.}

$(\Rightarrow)$ Suponhamos que $x^{*} \in \mathbb{R}^{n}$ e $U^{*} \in \mathbb{S}^{m}$ sejam soluções dos problemas primal e dual, respectivamente, e que a folga de dualidade seja zero. Assim, $A_{0}+\mathcal{A}\left(x^{*}\right) \preceq 0$ e $U^{*} \succeq 0$, o que prova as condições $(2.8 \mathrm{~b})$ e $(2.8 \mathrm{c})$.

Pela Proposição 2.3.1, sabemos que $\left(x^{*}, U^{*}\right)$ é um ponto de sela de L, ou seja,

$$
\begin{aligned}
& x^{*} \in \underset{x \in \mathbb{R}^{n}}{\operatorname{argmin}} \mathrm{L}\left(x, U^{*}\right) \quad \mathrm{e} \\
& U^{*} \in \underset{U \succeq 0}{\operatorname{argmax}} \mathrm{L}\left(x^{*}, U\right) .
\end{aligned}
$$

A partir da primeira expressão acima, temos

$$
\mathrm{L}\left(x, U^{*}\right) \geq \mathrm{L}\left(x^{*}, U^{*}\right)=\mathrm{L}\left(x^{*}, U^{*}\right)+\left\langle 0, x-x^{*}\right\rangle,
$$

para todo $x \in \mathbb{R}^{n}$, o que equivale a $0 \in \partial_{x} \mathrm{~L}\left(x^{*}, U^{*}\right)$. Assim, provamos a condição (2.8a).

Finalmente, provemos a condição (2.8d). Da expressão (2.9b), temos

$$
\mathrm{L}\left(x^{*}, U^{*}\right)=\sup _{U \succeq 0} \mathrm{~L}\left(x^{*}, U\right) .
$$

Já que $x^{*}$ é um ponto viável do problema primal, a partir da Hipótese 2.1 e da expressão (2.1), sabemos que $\sup _{U \succeq 0} \mathrm{~L}\left(x^{*}, U\right)=f\left(x^{*}\right)$ é finito. Logo,

$$
f\left(x^{*}\right)+\left\langle U^{*}, A_{0}+\mathcal{A}\left(x^{*}\right)\right\rangle=\mathrm{L}\left(x^{*}, U^{*}\right)=\sup _{U \succeq 0} \mathrm{~L}\left(x^{*}, U\right)=f\left(x^{*}\right) \in \mathbb{R},
$$

resultando que $\left\langle U^{*}, A_{0}+\mathcal{A}\left(x^{*}\right)\right\rangle=0$ e $x^{*}$ é viável em (PSDA), o que prova a condição $(2.8 \mathrm{~d})$.

$(\Leftarrow)$ Suponhamos que as condições $(2.8 \mathrm{a}),(2.8 \mathrm{~b}),(2.8 \mathrm{c})$ e $(2.8 \mathrm{~d})$ sejam válidas. Para toda matriz $U \in \mathbb{S}_{+}^{m}$, sabemos, pelo Corolário 1.3.7, que $\left\langle U, A_{0}+\mathcal{A}\left(x^{*}\right)\right\rangle \leq 0=\left\langle U^{*}, A_{0}+\right.$ $\left.\mathcal{A}\left(x^{*}\right)\right\rangle$. Logo,

$$
\mathrm{L}\left(x^{*}, U\right)=f\left(x^{*}\right)+\left\langle U, A_{0}+\mathcal{A}\left(x^{*}\right)\right\rangle \leq f\left(x^{*}\right)+\left\langle U^{*}, A_{0}+\mathcal{A}\left(x^{*}\right)\right\rangle=\mathrm{L}\left(x^{*}, U^{*}\right),
$$


resultando que

$$
\sup _{U \succeq 0} \mathrm{~L}\left(x^{*}, U\right)=\mathrm{L}\left(x^{*}, U^{*}\right) .
$$

Além disso, pela condição (2.8a), sabemos que

$$
\mathrm{L}\left(x, U^{*}\right) \geq \mathrm{L}\left(x^{*}, U^{*}\right)+\left\langle 0, x-x^{*}\right\rangle=\mathrm{L}\left(x^{*}, U^{*}\right) \text {, para todo } x \in \mathbb{R}^{n} \text {. }
$$

Tal inequação equivale a

$$
\inf _{x \in \mathbb{R}^{n}} \mathrm{~L}\left(x, U^{*}\right)=\mathrm{L}\left(x^{*}, U^{*}\right) .
$$

Logo, ao combinarmos as expressões $(2.10)$ e (2.11), podemos concluir que $\left(x^{*}, U^{*}\right)$ é um ponto de sela de L. Novamente pela Proposição 2.3.1, sabemos que $x^{*}$ e $U^{*}$ são soluções de (PSDA) e de (DSDA), respectivamente, e que a folga de dualidade é zero.

Observemos que se a função objetivo de (PSDA) for diferenciável, a função $L$ também será diferenciável em relação à primeira variável e admitirá um minimizador $x^{*}$ em relação ao primeiro argumento tal que $\nabla_{x} \mathrm{~L}\left(x^{*}, U^{*}\right)=0$. Logo, podemos escrever a condição (2.8a) como

$$
\begin{aligned}
0 & =\nabla_{x} \mathrm{~L}\left(x^{*}, U^{*}\right) \\
& =\nabla f\left(x^{*}\right)+\nabla_{x}\left(\left\langle U^{*}, A_{0}+\mathcal{A}\left(x^{*}\right)\right\rangle\right) \\
& =\nabla f\left(x^{*}\right)+\mathcal{A}^{*}\left(U^{*}\right) \quad \text { [Corolário 1.5.6]. }
\end{aligned}
$$

Ao considerarmos especificamente o problema primal linear, a condição (2.8a) pode ser definida como

$$
c+\mathcal{A}^{*}\left(U^{*}\right)=0
$$

que, junto com a condição (2.8c), definem o conjunto viável do problema dual linear. Logo, pelo Teorema 2.3.2, obtemos as condições de otimalidade abaixo para o problema primal linear.

Corolário 2.3.3 (Condições KKT para (PSDL)). Sejam $x^{*} \in \mathbb{R}^{n}$ e $U^{*} \in \mathbb{S}^{m}$. Então, $x^{*}$ é uma solução do problema primal linear, $U^{*}$ é uma solução do problema dual linear e a folga de dualidade é zero se e somente se

$$
\begin{aligned}
A_{0}+\mathcal{A}\left(x^{*}\right) \preceq 0 & & \text { (viabilidade primal), } \\
U^{*} \succeq 0 \text { e } c+\mathcal{A}^{*}\left(U^{*}\right)=0 & & \text { (viabilidade dual) } \\
\left\langle U^{*}, A_{0}+\mathcal{A}\left(x^{*}\right)\right\rangle=0 & & \text { (folgas complementares). }
\end{aligned}
$$

Geralmente, estamos interessados em obter soluções somente do problema primal ou do dual. A resolução do outro problema pode ser feita conjuntamente com a do problema de interesse, muitas vezes com baixo custo computacional, com o objetivo de fornecer informações úteis sobre as soluções ou o valor ótimo. Estudamos agora condições que 
garantem que a folga de dualidade é zero e que o conjunto de soluções de (DSDA) é nãovazio e compacto, mas, não necessariamente, que (PSDA) tem solução. Durante o texto, vamos denotar por

$$
\begin{aligned}
& \mathrm{F}_{\mathrm{P}} \stackrel{\text { def }}{=}\left\{x \in \mathbb{R}^{n} \mid A_{0}+\mathcal{A}(x) \preceq 0\right\}, \\
& \mathrm{F}_{\mathrm{P}}^{\circ} \stackrel{\text { def }}{=}\left\{x \in \mathrm{F}_{\mathrm{P}} \mid A_{0}+\mathcal{A}(x) \prec 0\right\}, \\
& \mathrm{F}_{\mathrm{D}} \stackrel{\text { def }}{=}\left\{U \in \mathbb{S}^{m} \mid U \succeq 0\right\}, \\
& \mathrm{F}_{\mathrm{D}} \stackrel{\text { def }}{=}\left\{U \in \mathrm{F}_{\mathrm{D}} \mid U \succ 0\right\}
\end{aligned}
$$

os conjuntos de pontos viáveis e estritamente viáveis de (PSDA) e (DSDA), respectivamente. Além disso, sejam $S_{P}^{*}$ e $S_{D}^{*}$ os conjuntos de soluções de (PSDA) e de (DSDA), respectivamente.

De modo similar ao feito em otimização não linear restrita no $\mathbb{R}^{n}$, dizemos que a condição de qualificação de Slater é válida para o problema primal se existe $\bar{x} \in \operatorname{dom} f$ tal que $A_{0}+\mathcal{A}(\bar{x}) \prec 0$, isto é, se $\mathrm{F}_{\mathrm{P}}^{\circ} \neq \emptyset$.

A seguir, provamos que, sob a hipótese do valor ótimo do problema primal ser finito, a condição de qualificação de Slater é válida se e somente se o conjunto de soluções do problema dual associado é não-vazio e compacto. É importante ressaltar que esse resultado é conhecido e a idéia de sua demonstração é sugerida em [DT99]. Entretanto, não encontramos nenhuma prova explícita nas referências estudadas. Por esse motivo, decidiu-se incluir tal demonstração neste texto. A prova baseia-se nas idéias discutidas em [DT99], [Sil00, Teorema 1.2.5], [Ber03, Capítulo 6] e [Roc70, Corolário 28.2.1].

Teorema 2.3.4. Suponhamos que o valor ótimo do problema primal seja finito. Então, a condição de qualificação de Slater é válida para (PSDA), ou seja, existe $\tilde{x} \in \operatorname{dom} f$ tal que

$$
A_{0}+\mathcal{A}(\tilde{x}) \prec 0,
$$

se e somente se a folga de dualidade é zero e o conjunto de soluções do problema dual é não-vazio e compacto.

Demonstração.

$(\Rightarrow)$ Consideremos que a condição de Slater seja válida para (PSDA). Pelo fato de $\mathrm{f}_{*}^{\mathrm{P}}$ ser finito, sabemos que o sistema

$$
\begin{aligned}
& f(x)-\mathrm{f}_{*}^{\mathrm{P}}<0, \\
& A_{0}+\mathcal{A}(x) \prec 0, \\
& x \in \operatorname{dom} f
\end{aligned}
$$

não tem solução.

Desta forma, pelo Teorema B.1, para $C=\operatorname{dom} f$, existem $\tilde{\lambda} \in \mathbb{R}_{+}$e $\tilde{U} \in \mathbb{S}_{+}^{m}$ tais que

$$
\tilde{\lambda}\left(f(x)-\mathrm{f}_{*}^{\mathrm{P}}\right)+\left\langle\tilde{U}, A_{0}+\mathcal{A}(x)\right\rangle \geq 0, \quad \text { para todo } x \in \operatorname{dom} f,
$$

e, além disso, $\tilde{\lambda}>0$ ou $\tilde{U} \neq 0$.

Suponhamos que $\tilde{\lambda}=0$. Então, $\tilde{U} \neq 0$ e, para todo $x \in \operatorname{dom} f$, temos

$$
\left\langle\tilde{U}, A_{0}+\mathcal{A}(x)\right\rangle \geq 0,
$$


o que é uma contradição, visto que, por hipótese, existe $\tilde{x} \in \operatorname{dom} f$ tal que $A_{0}+\mathcal{A}(\tilde{x}) \prec 0$, implicando que $\left\langle\tilde{U}, A_{0}+\mathcal{A}(\tilde{x})\right\rangle<0$ pela Proposição 1.3.8. Logo, nós concluímos que $\tilde{\lambda}>0$ e podemos supor, sem perda de generalidade, que $\tilde{\lambda}=1$. De fato, basta dividirmos a inequação $(2.13)$ por $\tilde{\lambda}$.

A partir de (2.13), vemos que $f(x)+\left\langle\tilde{U}, A_{0}+\mathcal{A}(x)\right\rangle \geq \mathrm{f}_{*}^{\mathrm{P}}$ para todo $x \in \operatorname{dom} f$. Logo,

$$
\begin{aligned}
\inf _{x \in \mathbb{R}^{n}} \mathrm{~L}(x, \tilde{U}) & =\inf _{x \in \mathbb{R}^{n}}\left\{f(x)+\left\langle\tilde{U}, A_{0}+\mathcal{A}(x)\right\rangle\right\} \\
& =\inf _{x \in \operatorname{dom} f}\left\{f(x)+\left\langle\tilde{U}, A_{0}+\mathcal{A}(x)\right\rangle\right\} \\
& \geq \mathrm{f}_{*}^{\mathrm{P}},
\end{aligned}
$$

resultando que

$$
\mathrm{f}_{*}^{\mathrm{D}}=\sup _{U \in \mathbb{S}_{+}^{m}} \inf _{x \in \mathbb{R}^{n}} \mathrm{~L}(x, U) \geq \mathrm{f}_{*}^{\mathrm{P}}
$$

Por outro lado, pelo Corolário 2.2.2, sabemos que $\mathrm{f}_{*}^{\mathrm{D}} \leq \mathrm{f}_{*}^{\mathrm{P}}$. Assim, a folga de dualidade é zero e $\inf _{x \in \mathbb{R}^{n}} \mathrm{~L}(x, \tilde{U})=\sup _{U \in \mathbb{S}_{+}^{m}} \inf _{x \in \mathbb{R}^{n}} \mathrm{~L}(x, U)$, o que implica em $\tilde{U}$ ser uma solução do problema dual. Desta forma, $\mathrm{S}_{\mathrm{D}}^{*}$ é não-vazio. Provemos agora que $\mathrm{S}_{\mathrm{D}}^{*}$ é compacto.

$\mathrm{O}$ conjunto $\mathrm{S}_{\mathrm{D}}^{*}$ é fechado, pois é um conjunto de nível não-vazio da função côncava fechada $\inf _{x \in \mathbb{R}^{n}} \mathrm{~L}(x, \cdot)$ definida sobre $\mathbb{S}^{m}$. Resta provarmos que $\mathrm{S}_{\mathrm{D}}^{*}$ é limitado.

Suponhamos que $\mathrm{S}_{\mathrm{D}}^{*}$ não seja limitado e seja $U^{*} \in \mathrm{S}_{\mathrm{D}}^{*}$. Então, existe $H \in \mathbb{S}^{m}$ tal que $H \neq 0$ e $U^{*}+\alpha H \in \mathrm{S}_{\mathrm{D}}^{*}$, para todo $\alpha \geq 0$ (veja [BL06, pág. 6, exercício 6] e [Ber03, Proposição 1.5.1]). Já que a folga de dualidade é zero, obtemos

$$
\inf _{x \in \mathbb{R}^{n}} \mathrm{~L}\left(x, U^{*}+\alpha H\right)=\mathrm{f}_{*}^{\mathrm{P}}, \quad \text { para todo } \alpha \geq 0 .
$$

Também suponhamos que $H \nsucceq 0$. Então, existe $\bar{x} \in \mathbb{R}^{n}$ tal que $\bar{x}^{T} H \bar{x}<0$. Desta forma, para $\bar{\alpha}$ estritamente positivo e suficientemente grande, obtemos $\bar{x}\left(U^{*}+\bar{\alpha} H\right) \bar{x}<0$, o que contraria nossa hipótese de $U^{*}+\alpha H$ ser uma solução de (DSDA) para todo $\alpha \geq 0$. Portanto, $H \succeq 0$.

Além disso, para todo $\alpha \geq 0$, obtemos

$$
\begin{aligned}
\mathrm{L}\left(\tilde{x}, U^{*}+\alpha H\right) & =f(\tilde{x})+\left\langle U^{*}+\alpha H, A_{0}+\mathcal{A}(\tilde{x})\right\rangle \\
& =f(\tilde{x})+\left\langle U^{*}, A_{0}+\mathcal{A}(\tilde{x})\right\rangle+\alpha\left\langle H, A_{0}+\mathcal{A}(\tilde{x})\right\rangle .
\end{aligned}
$$

Devido à hipótese de $H \neq 0$, pela Proposição 1.3.8, sabemos que $\left\langle H, A_{0}+\mathcal{A}(\tilde{x})\right\rangle<0$. Assim, para $\alpha \rightarrow+\infty$, temos $\mathrm{L}(\tilde{x}, U+\alpha H) \rightarrow-\infty$, implicando que $\inf _{x \in \mathbb{R}^{n}} \mathrm{~L}(x, U) \rightarrow-\infty$ também. E, por (2.14), concluímos que $\mathrm{f}_{*}^{\mathrm{P}}=-\infty$, o que contradiz a hipótese de $\mathrm{f}_{*}^{\mathrm{P}}$ ser finito. Portanto, $\mathrm{S}_{\mathrm{D}}^{*}$ é limitado e, já que também é fechado, sabemos que é compacto.

$(\Leftarrow)$ Consideremos que o conjunto $\mathrm{S}_{\mathrm{D}}^{*}$ seja não-vazio e compacto e que a folga de dualidade seja zero. Seja $U^{*} \succeq 0$ uma solução do problema dual. Suponhamos que a condição de Slater não seja válida, isto é,

$$
A_{0}+\mathcal{A}(x) \nprec 0 \text { para todo } x \in \operatorname{dom} f \text {. }
$$


A partir do Teorema B.1, sabemos que existe uma matriz $H \succeq 0$, não-nula, tal que

$$
\left\langle H, A_{0}+\mathcal{A}(x)\right\rangle \geq 0, \text { para todo } x \in \operatorname{dom} f .
$$

Logo, para todo real $\alpha \geq 0$ e todo $x \in \operatorname{dom} f$,

$$
\begin{aligned}
\mathrm{L}\left(x, U^{*}+\alpha H\right) & =f(x)+\left\langle U^{*}+\alpha H, A_{0}+\mathcal{A}(x)\right\rangle \\
& =f(x)+\left\langle U^{*}, A_{0}+\mathcal{A}(x)\right\rangle+\alpha\left\langle H, A_{0}+\mathcal{A}(x)\right\rangle \\
& \geq f(x)+\left\langle U^{*}, A_{0}+\mathcal{A}(x)\right\rangle \\
& =\mathrm{L}\left(x, U^{*}\right),
\end{aligned}
$$

implicando que

$$
\mathrm{L}\left(x, U^{*}+\alpha H\right) \geq \mathrm{L}\left(x, U^{*}\right) .
$$

Ao tomarmos o ínfimo em relação a $x$ nos dois lados da inequação acima, obtemos a expressão

$$
\inf _{x \in \operatorname{dom} f} \mathrm{~L}\left(x, U^{*}+\alpha H\right) \geq \inf _{x \in \operatorname{dom} f} \mathrm{~L}\left(x, U^{*}\right),
$$

a qual equivale a

$$
\inf _{x \in \mathbb{R}^{n}} \mathrm{~L}\left(x, U^{*}+\alpha H\right) \geq \inf _{x \in \mathbb{R}^{n}} \mathrm{~L}\left(x, U^{*}\right) .
$$

Então, $U^{*}+\alpha H$ também é solução do problema dual, para todo $\alpha \geq 0$. Já que podemos escolher $\alpha$ arbitrariamente grande, segue que o conjunto de soluções do problema dual é ilimitado, o que contradiz nossa hipótese. Portanto, a condição de Slater é válida.

Consideremos o problema dual linear em particular. Representaremos por

$$
\begin{aligned}
& \mathrm{F}_{\mathrm{DL}} \stackrel{\text { def }}{=}\left\{U \in \mathrm{F}_{\mathrm{D}} \mid U \succeq 0 \text { e } c+\mathcal{A}^{*}(U)=0\right\}, \\
& \mathrm{F}_{\mathrm{DL}}^{\circ} \stackrel{\text { def }}{=}\left\{U \in \mathrm{F}_{\mathrm{DL}} \mid U \succ 0\right\}
\end{aligned}
$$

os conjuntos de pontos viáveis e estritamente viáveis do problema dual linear, respectivamente. Ademais, seja $S_{D L}^{*}$ o conjunto de soluções desse problema. A partir do que foi discutido no início deste capítulo, se o problema lagrangiano dual possui valor ótimo maior que $-\infty$, então $\mathrm{S}_{\mathrm{D}}^{*}=\mathrm{S}_{\mathrm{DL}}^{*}$. Assim, a partir do Teorema 2.3.4, temos que a condição de Slater é válida para (PSDL) se e somente se $\mathrm{S}_{\mathrm{DL}}^{*}$ é não-vazio e compacto. 


\section{Capítulo 3}

\section{Um algoritmo proximal interior e o método de multiplicadores exponenciais para programação semidefinida}

Neste capítulo, apresentamos um método de ponto proximal para resolver o problema dual introduzido no capítulo anterior. Também abordamos o método de multiplicadores associado a esse método de ponto proximal para resolver o problema primal.

\subsection{Algoritmo de ponto proximal interior}

No capítulo anterior, introduzimos o problema dual associado ao problema primal:

$$
\sup _{U \in \mathbb{S}_{+}^{m}} \inf _{x \in \mathbb{R}^{n}} \mathrm{~L}(x, U) \text {. }
$$

Vimos que o valor ótimo desse problema é um limitante inferior do valor ótimo do problema primal e que, se algumas condições são satisfeitas, então os dois problemas têm o mesmo valor ótimo. Assim, caso a resolução do problema dual seja mais fácil que a do problema primal, torna-se interessante resolvê-lo para obter-se uma estimativa inferior do valor ótimo do problema primal ou, possivelmente, o próprio valor ótimo. Além disso, em algumas situações, é possível obter-se uma solução do problema primal a partir de uma solução do problema dual, como é descrito posteriormente neste capítulo.

Obter uma solução de (DSDA) é equivalente a obter uma solução do problema

$$
\inf _{U \in \mathbb{S}_{+}^{m}}\left\{-\mathcal{K}_{\mathrm{D}}(U)\right\}
$$

cujo valor ótimo é $-\mathrm{f}_{*}^{\mathrm{D}}$. Dessa forma, o problema dual é um caso particular do seguinte problema de minimização, o qual denotamos por (DM):

$$
\begin{array}{cl}
\min & g(U) \\
\text { s. a } & U \succeq 0, \\
& U \in \mathbb{S}^{m},
\end{array}
$$


em que $g: \mathbb{S}^{m} \rightarrow(-\infty,+\infty]$ é uma função matricial convexa e fechada. Em particular, em relação ao problema dual, obtemos $g(U)=-\mathcal{K}_{\mathrm{D}}(U)$. Além disso, se $\mathcal{K}_{\mathrm{D}}(\tilde{U})>-\infty$ para alguma matriz $\tilde{U} \in \mathbb{S}^{m}$, então a função objetivo de (DM) também é própria.

É importante destacar que o problema dual linear também é um caso particular de (DM). Nesse caso, tomamos $g$ por

$$
g(U)=-\left\langle U, A_{0}\right\rangle+\delta_{\left\{U \in \mathbb{S}^{m} \mid c+\mathcal{A}^{*}(U)=0\right\}}(U) .
$$

Tal função é convexa e fechada, pois é a soma de duas funções convexas e fechadas. Ademais, se (DSDL) admite algum ponto viável, então $g$ é própria. Tal condição é equivalente a $\mathcal{K}_{\mathrm{D}}(\tilde{U})>-\infty$ para alguma matriz $\tilde{U} \in \mathbb{S}^{m}$, conforme vimos no Capítulo 2.

Para resolver (DM), o seguinte algoritmo de ponto proximal interior (API) foi introduzido por Doljansky e Teboulle em [DT99, Seção 3]:

\section{Algoritmo 3.1. (API)}

(i) Escolha uma matriz inicial $U_{0} \in \mathbb{S}_{++}^{m}$.

(ii) Para $k \geq 1$, gere a seqüência $\left\{U_{k}\right\}$ que satisfaz

$$
U_{k}=\underset{U \in \mathbb{S}_{+}^{m}}{\operatorname{argmin}}\left\{g(U)+\frac{1}{\mu_{k}} \mathrm{D}_{\mathrm{DT}}\left(U, U_{k-1}\right)\right\},
$$

em que $\mu_{k}>0$ é um parâmetro de regularização e $\mathrm{D}_{\mathrm{DT}}: \mathbb{S}^{m} \times \mathbb{S}^{m} \rightarrow(-\infty,+\infty]$ é a regularização definida por

$$
\mathrm{D}_{\mathrm{DT}}(U, V) \stackrel{\text { def }}{=} \begin{cases}\operatorname{tr}(U \ln U-U \ln V+V-U) & \text { se }(U, V) \in \mathbb{S}_{+}^{m} \times \mathbb{S}_{++}^{m}, \\ +\infty & \text { caso contrário. }\end{cases}
$$

No texto, consideramos que $U \ln U=0$ para $U=0$. Assim, para $V \in \mathbb{S}_{++}^{m}$, obtemos $\mathrm{D}_{\mathrm{DT}}(0, V)=\operatorname{tr}(V)$.

Esse algoritmo tem como regularização um funcional $\mathrm{D}_{\mathrm{DT}}$ que garante que a seqüência de matrizes $U_{k}$ geradas está contida no interior do cone convexo $\mathbb{S}_{+}^{m}$, conforme está provado posteriormente. Ou seja, todas as matrizes da seqüência $\left\{U_{k}\right\}$ são pontos viáveis de (DM).

Vale ressaltar que o método proposto por Doljansky e Teboulle estende resultados existentes relacionados a métodos proximais entrópicos em programação convexa no $\mathbb{R}^{n}$. De fato, a regularização $\mathrm{D}_{\mathrm{DT}}$ pode ser vista como uma extensão da entropia relativa associada à distância de Kullback-Leibler, uma função escalar $\mathrm{D}_{\mathrm{KL}}$ definida em $\mathbb{R}_{+}^{n} \times \mathbb{R}_{++}^{n}$ por

$$
\mathrm{D}_{\mathrm{KL}}(a, b) \stackrel{\text { def }}{=} \sum_{i=1}^{n} \mathrm{~d}_{\mathrm{KL}}\left(a_{i}, b_{i}\right)=\sum_{i=1}^{n}\left(a_{i} \ln a_{i}-a_{i} \ln b_{i}+b_{i}-a_{i}\right) .
$$

Assim, podemos considerar a função $\mathrm{D}_{\mathrm{DT}}$ como uma medida da "distância" entre duas matrizes no cone $\mathbb{S}_{+}^{m}$.

Abordamos agora algumas propriedades conhecidas da distância $\mathrm{D}_{\mathrm{KL}}$ aplicadas à regularização $\mathrm{D}_{\mathrm{DT}}$ e que são usadas para demonstrar a convergência do algoritmo apresentado. Para simplificar a notação usada neste capítulo, denotamos a regularização $\mathrm{D}_{\mathrm{DT}}$ por $\mathrm{D}$ e a função $\mathrm{D}_{\mathrm{KL}}$ por $d$. 
Lema 3.1.1 ([DT99, Lema 3.1]). As seguintes afirmações são válidas para a regularização D:

(i) D é uma função contínua sobre $\mathbb{S}_{+}^{m} \times \mathbb{S}_{++}^{m}$ e $U \rightarrow \mathrm{D}(U, V)$ é uma função estritamente convexa para toda matriz $V \in \mathbb{S}_{++}^{m}$.

(ii) $\mathrm{D}(U, V) \geq 0$ para todo $(U, V) \in \mathbb{S}_{+}^{m} \times \mathbb{S}_{++}^{m} e \mathrm{D}(U, V)=0$ se e somente se $U=V$.

(iii) $\mathrm{D}(U, V) \geq d(\lambda(U), \lambda(V))$ para todo $(U, V) \in \mathbb{S}_{+}^{m} \times \mathbb{S}_{++}^{m}$.

(iv) Os conjuntos de nível de $\mathrm{D}(\cdot, V)$ e $\mathrm{D}(U, \cdot)$ são limitados para todas as matrizes $V \in$ $\mathbb{S}_{++}^{m}$ e $U \in \mathbb{S}_{+}^{m}$, respectivamente.

Demonstração.

(i) A partir de [HJ94, Capítulo 6], do Corolário 1.5.12 e da Proposição 1.5.5, sabemos que as funções $U \ln U$ e $\ln V$ são contínuas sobre $\mathbb{S}_{+}^{m}$ e $\mathbb{S}_{++}^{m}$, respectivamente. Assim, D é contínua sobre $\mathbb{S}_{+}^{m} \times \mathbb{S}_{++}^{m}$. Pelo Teorema 1.5.13, a função $\operatorname{tr}(U \ln U)$ é estritamente convexa sobre $\mathbb{S}_{+}^{m}$, resultando que $U \rightarrow \mathrm{D}(U, V)$ é estritamente convexa para todo $V \in \mathbb{S}_{++}^{m} \cdot$

(ii) Seja $\psi(U) \stackrel{\text { def }}{=} \operatorname{tr}(U \ln U)=\langle U, \ln U\rangle$. Obtemos $\nabla \psi(U)=\ln U+I$ a partir do Corolário 1.5.12. Já que $\psi$ é estritamente convexa, a desigualdade do gradiente (Proposição 1.4.8) é válida para todo $(U, V) \in \mathbb{S}_{+}^{m} \times \mathbb{S}_{++}^{m}$ :

$$
\begin{aligned}
& \psi(U)-\psi(V) \geq\langle U-V, \nabla \psi(V)\rangle \\
& \Leftrightarrow \quad\langle U, \ln U\rangle-\langle V, \ln V\rangle \geq\langle U-V, \ln V+I\rangle \\
& \Leftrightarrow \quad\langle U, \ln U\rangle-\langle V, \ln V\rangle \geq\langle U, \ln V\rangle-\langle V, \ln V\rangle+\langle U, I\rangle-\langle V, I\rangle \\
& \Leftrightarrow \quad \operatorname{tr}(U \ln U)-\operatorname{tr}(U \ln V)-\operatorname{tr} U+\operatorname{tr} V \geq 0 \\
& \Leftrightarrow \quad \mathrm{D}(U, V) \geq 0,
\end{aligned}
$$

provando a primeira parte do item $(i i)$. Além disso, devido a $\psi$ ser estritamente convexa, a desigualdade estrita é válida na inequação acima se $U \neq V$.

(iii) A partir da definição de função matricial feita no Capítulo 1, sabemos que $\lambda_{i}(\ln V)=$ $\ln \lambda_{i}(V)$, para $V \in \mathbb{S}_{++}^{m}$ e $i=1, \ldots, m$ e, pelo Teorema 1.2.4, obtemos

$$
\operatorname{tr}(U \ln V) \leq \sum_{i=1}^{n} \lambda_{i}(U) \lambda_{i}(\ln V)=\sum_{i=1}^{n} \lambda_{i}(U) \ln \lambda_{i}(V)
$$

para todo $(U, V) \in \mathbb{S}_{+}^{m} \times \mathbb{S}_{++}^{m}$. A partir dessa desigualdade e da definição da regula- 
rização D, sabemos que

$$
\begin{aligned}
\mathrm{D}(U, V) & =\operatorname{tr}(U \ln U-U \ln V+V-U) \\
& =\operatorname{tr}(U \ln U+V-U)-\operatorname{tr}(U \ln V) \\
& \geq \operatorname{tr}(U \ln U+V-U)-\sum_{i=1}^{m} \lambda_{i}(U) \ln \lambda_{i}(V) \\
& =\operatorname{tr}(U \ln U)+\operatorname{tr}(V)-\operatorname{tr}(U)-\sum_{i=1}^{m} \lambda_{i}(U) \ln \lambda_{i}(V) \\
& =\sum_{i=1}^{m} \lambda_{i}(U) \ln \lambda_{i}(U)+\operatorname{tr}(V)-\operatorname{tr}(U)-\sum_{i=1}^{m} \lambda_{i}(U) \ln \lambda_{i}(V) \quad \text { Proposição1.5.4] } \\
& =\sum_{i=1}^{m}\left(\lambda_{i}(U) \ln \lambda_{i}(U)+\lambda_{i}(V)-\lambda_{i}(U)-\lambda_{i}(U) \ln \lambda_{i}(V)\right) \\
& =d(\lambda(U), \lambda(V)) .
\end{aligned}
$$

Logo, a partir da expressão acima, temos $\mathrm{D}(U, V) \geq d(\lambda(U), \lambda(V))$.

(iv) Para todo escalar $\eta \geq 0$ e toda matriz $V \in \mathbb{S}_{++}^{m}$, sejam

$$
\begin{aligned}
& z(V, \eta) \stackrel{\text { def }}{=}\left\{U \in \mathbb{S}_{+}^{m} \mid \mathrm{D}(U, V) \leq \eta\right\}, \\
& \mathcal{J}(V, \eta) \stackrel{\text { def }}{=}\left\{U \in \mathbb{S}_{+}^{m} \mid d(\lambda(U), \lambda(V)) \leq \eta\right\} .
\end{aligned}
$$

A partir do item $(i i i)$, temos que $z(V, \eta) \subseteq \mathcal{J}(V, \eta)$. Devido a $d(\cdot, b)$ ter conjuntos de nível limitados para todo $b>0$, sabemos que os autovalores das matrizes de $\mathcal{J}(V, \eta)$ são limitados também, resultando que $\mathcal{J}(V, \eta)$ e $\mathcal{Z}(V, \eta)$ são limitados. De maneira análoga, ao repetirmos as idéias dessa demonstração, temos que $\mathrm{D}(U, \cdot)$ tem conjuntos de nível limitados para toda matriz $U \in \mathbb{S}_{+}^{m}$.

Lema 3.1.2 ([DT99, Lema 3.2]). Sejam $\left\{U_{k}\right\} \subseteq \mathbb{S}_{+}^{m}$ e $\left\{V_{k}\right\} \subseteq \mathbb{S}_{++}^{m}$ seqüências limitadas que satisfaçam $\mathrm{D}\left(U_{k}, V_{k}\right) \rightarrow 0$ quando $k \rightarrow \infty$. Então, as seguintes afirmações são válidas quando $k \rightarrow \infty$ :

(i) $\lambda\left(U_{k}\right)-\lambda\left(V_{k}\right) \rightarrow 0$.

(ii) $\operatorname{tr}\left(U_{k}-V_{k}\right) \rightarrow 0$.

\section{Demonstração.}

(i) Devido à hipótese das seqüências $\left\{U_{k}\right\}$ e $\left\{V_{k}\right\}$ serem limitadas, sabemos que as seqüências $\left\{\lambda_{i}\left(U_{k}\right)\right\}$ e $\left\{\lambda_{i}\left(V_{k}\right)\right\}$ são limitadas também, para $i=1, \ldots, m$. Ademais, a partir do Lema 3.1.1 (iii), temos que $\mathrm{D}\left(U_{k}, V_{k}\right) \geq d\left(\lambda\left(U_{k}\right), \lambda\left(V_{k}\right)\right) \geq 0$. Assim, pela hipótese de $\mathrm{D}\left(U_{k}, V_{k}\right) \rightarrow 0$ quando $k \rightarrow \infty$, temos $d\left(\lambda\left(U_{k}\right), \lambda\left(V_{k}\right)\right) \rightarrow 0$ também. A partir da definição da entropia relativa de Kullback-Leibler feita na equação (3.3), sabemos que $d\left(\lambda\left(U_{k}\right), \lambda\left(V_{k}\right)\right)=\sum_{i=1}^{m} d_{\mathrm{KL}}\left(\lambda_{i}\left(U_{k}\right), \lambda_{i}\left(V_{k}\right)\right)$, resultando que $\mathrm{d}_{\mathrm{KL}}\left(\lambda_{i}\left(U_{k}\right), \lambda_{i}\left(V_{k}\right)\right) \rightarrow 0$, para $i=1, \ldots, m$. Logo, pela Lema A.1, obtemos que $\left(\lambda_{i}\left(U_{k}\right)-\lambda_{i}\left(V_{k}\right)\right) \rightarrow 0$, de onde segue o resultado desejado. 
(ii) Já que $\operatorname{tr}(U)=\sum_{i=1}^{m} \lambda_{i}(U)$ e $\operatorname{tr}(U-V)=\operatorname{tr}(U)-\operatorname{tr}(V)$, para quaisquer matrizes $U, V \in \mathbb{S}^{m}$, obtemos

$$
\operatorname{tr}\left(U_{k}-V_{k}\right)=\operatorname{tr}\left(U_{k}\right)-\operatorname{tr}\left(V_{k}\right)=\sum_{i=1}^{m} \lambda_{i}\left(U_{k}\right)-\sum_{i=1}^{m} \lambda_{i}\left(V_{k}\right)=\sum_{i=1}^{m}\left(\lambda_{i}\left(U_{k}\right)-\lambda_{i}\left(V_{k}\right)\right)
$$

para todo inteiro $k \geq 0$. Pelo item $(i)$, temos que $\operatorname{tr}\left(U_{k}-V_{k}\right) \rightarrow 0$ quando $k \rightarrow \infty$.

Lema 3.1.3 (Lema dos três pontos [DT99, Lema 3.3]). Para todas as matrizes $V, W \in \mathbb{S}_{++}^{m}$ e $U \in \mathbb{S}_{+}^{m}$, temos que:

(i) $\mathrm{D}(U, V)-\mathrm{D}(U, W)=\operatorname{tr}(U \ln W-U \ln V+V-W)$;

(ii) $\langle U-W, \ln W-\ln V\rangle=\mathrm{D}(U, V)-\mathrm{D}(U, W)-\mathrm{D}(W, V)$

$$
\leq \mathrm{D}(U, V)-\mathrm{D}(U, W) \text {. }
$$

Demonstração. A partir da definição da regularização D, temos

$$
\begin{aligned}
\mathrm{D}(U, V)-\mathrm{D}(U, W) & =\operatorname{tr}(U \ln U-U \ln V+V-U)-\operatorname{tr}(U \ln U-U \ln W+W-U) \\
& =\operatorname{tr}(U \ln W-U \ln V+V-W),
\end{aligned}
$$

o que prova o item (i). Provemos agora o item (ii).

Novamente a partir da definição de D, temos

$$
\begin{aligned}
\mathrm{D}(U, V)-\mathrm{D}(U, W)-\mathrm{D}(W, V) & =\operatorname{tr}(U \ln U-U \ln V+V-U) \\
& -\operatorname{tr}(U \ln U-U \ln W+W-U) \\
& -\operatorname{tr}(W \ln W-W \ln V+V-W) \\
& =\operatorname{tr}(-U \ln V+U \ln W-W \ln W+W \ln V) \\
& =\operatorname{tr}((U-W)(\ln W-\ln V)) \\
& =\langle U-W, \ln W-\ln V\rangle,
\end{aligned}
$$

o que prova a igualdade do item (ii). Além disso, pelo Lema 3.1.1(ii), $\mathrm{D}(W, V) \geq 0$, implicando que $\mathrm{D}(U, V)-\mathrm{D}(U, W)-\mathrm{D}(W, V) \leq \mathrm{D}(U, V)-\mathrm{D}(U, W)$, o que completa a prova.

\subsubsection{Análise de convergência do algoritmo proximal}

A análise de convergência do algoritmo proximal interior apresentado na seção anterior é feita com base na seqüência de valores da função objetivo de (DM) avaliada sobre a seqüencia de matrizes geradas pelo algoritmo.

Sejam $g^{*} \stackrel{\text { def }}{=} \inf _{U \in \mathbb{S}_{+}^{m}}\{g(U)\}$ e $\mathrm{S}_{\mathrm{DM}}^{*} \stackrel{\text { def }}{=}\left\{U \in \mathbb{S}_{+}^{m} \mid g(U)=g^{*}\right\}$ o valor ótimo e o conjunto de soluções de $(\mathrm{DM})$, respectivamente. Adotamos a convenção de que $g^{*}=-\infty$ se o problema for ilimitado inferiormente.

Para fazermos a análise de convergência, supomos que as seguintes hipóteses são válidas para o problema (DM): 
Hipótese 3.1.1. A função objetivo g é própria.

Hipótese 3.1.2. O valor ótimo do problema é limitado inferiormente, ou seja, $g^{*}>-\infty$.

Hipótese 3.1.3. O problema tem um ponto estritamente viável, ou seja, $\operatorname{dom} g \cap \mathbb{S}_{++}^{m} \neq \emptyset$.

Observemos que as Hipóteses 3.1.1 e 3.1.2 implicam que o valor ótimo de (DM) é finito.

Abordamos agora um resultado que garante que o Algoritmo (API) está bem definido.

Lema 3.1.4 ([DT99, Lema 4.1]). Para toda matriz $V \in \mathbb{S}_{++}^{m}$ e todo escalar $\mu \geq 0$, temos:

(i) se as Hipóteses 3.1.1 e 3.1.2 são satisfeitas, então a função $U \rightarrow g(U)+\mu^{-1} \mathrm{D}(U, V)$ tem conjuntos de nível limitados;

(ii) se as Hipóteses 3.1.1, 3.1.2 e 3.1 .3 são satisfeitas, então há uma única matriz $U(V) \in$ $\mathbb{S}_{++}^{m}$ tal que

$$
U(V)=\underset{U \in \mathbb{S}_{+}^{m}}{\operatorname{argmin}}\left\{g(U)+\frac{1}{\mu} \mathrm{D}(U, V)\right\}
$$

e que, portanto, satisfaz

$$
\mu^{-1}(\ln V-\ln U(V)) \in \partial g(U(V)) .
$$

\section{Demonstração.}

Seja $G_{V}: \mathbb{S}^{m} \rightarrow[-\infty,+\infty]$ definida por $G_{V}(U) \stackrel{\text { def }}{=} g(U)+\mu^{-1} \mathrm{D}(U, V)$. Suponhamos que a Hipóteses 3.1.1 e 3.1.2 sejam satisfeitas. A partir do Lema 3.1.1(iv), sabemos que os conjuntos de nível de $\mathrm{D}(\cdot, V)$ são limitados. Assim, já que $g$ é limitada inferiormente e própria, a função $G_{V}$ tem conjuntos de nível limitados, o que prova o item (i).

Suponhamos agora que as Hipóteses 3.1.1, 3.1.2 e 3.1.3 sejam satisfeitas. Pelo item (i), existe uma matriz $U(V)$ que minimiza a função $G_{V}$ em $\mathbb{S}_{+}^{m}$. Além disso, essa matriz é única, já que a função $\mathrm{D}(\cdot, V)$ é estritamente convexa, implicando que $G_{V}$ também é estritamente convexa.

Pela Proposição 1.4.6, sabemos que uma condição de otimalidade necessária e suficiente para que $U(V)$ satisfaça a equação (3.4) é dada por

$$
\begin{aligned}
0 & \in \partial\left(G_{V}(U(V))+\delta_{\mathbb{S}_{+}^{m}}(U(V))\right) \\
& =\partial_{U}\left(g(U(V))+\frac{1}{\mu} \mathrm{D}(U(V), V)+\delta_{\mathbb{S}_{+}^{m}}(U(V))\right) .
\end{aligned}
$$

Sob as Hipóteses 3.1.1 e 3.1.3, sabemos que

$$
\begin{aligned}
& \partial_{U}\left(g(U(V))+\frac{1}{\mu} \mathrm{D}(U(V), V)+\delta_{\mathbb{S}_{+}^{m}}(U(V))\right) \\
= & \partial g(U(V))+\frac{1}{\mu} \partial \mathrm{D}(U(V), V)+\partial \delta_{\mathbb{S}_{+}^{m}}(U(V)),
\end{aligned}
$$

pela regra da soma de subdiferenciais ([Roc70, Teorema 23.8]). Logo, a expressão (3.6) equivale a

$$
0 \in \partial g(U(V))+\mu^{-1} \partial \mathrm{D}(U(V), V)+\partial \delta_{\mathbb{S}_{+}^{m}}(U(V)) .
$$


A partir da definição de função matricial e da Proposição 1.5.5, sabemos que

$$
\left\|\nabla_{U} \mathrm{D}\left(U_{k}, V\right)\right\|=\left\|\ln U_{k}\right\| \rightarrow-\infty,
$$

para qualquer seqüência $\left\{U_{k}\right\} \subseteq \mathbb{S}_{++}^{m}$ tal que $U_{k} \rightarrow \bar{U} \in \mathbb{S}_{+}^{m} \backslash \mathbb{S}_{++}^{m}$, ou seja, para qualquer seqüência que convirja para a borda do cone de matrizes simétricas semidefinidas positivas. Assim, podemos afirmar que $\mathrm{D}(\cdot, Y)$ é uma função essencialmente suave (veja [Roc70, Seção $26])$. Logo, $\partial \mathrm{D}(U, V)=\emptyset$ para qualquer matriz simétrica semidefinida positiva e singular $U$, resultando que $U(V) \succ 0$. Além disso, a partir da Proposição 1.4 .9 e da Proposição 1.3.8, temos $\partial \delta_{\mathbb{S}_{+}^{m}}(U(V))=\{0\}$. Então, já que $\nabla_{U} \mathrm{D}(U, V)=\ln U-\ln V$, para toda $U \in \mathbb{S}_{+}^{m}$, a expressão (3.7) pode ser escrita como

$$
0 \in \partial g(U(V))+\mu^{-1}(\ln U(V)-\ln V)
$$

a qual equivale à expressão (3.5), o que conclui a demonstração.

O lema a seguir trata de algumas propriedades da seqüência $\left\{g\left(U_{k}\right)\right\}$ e da regularização D que serão usadas na análise de convergência do algoritmo.

Lema 3.1.5 ([DT99, Lema 4.2]). Sejam $\left\{U_{k}\right\}$ a seqüência gerada pelo Algoritmo (API) e $\sigma_{s} \stackrel{\text { def }}{=} \sum_{i=1}^{s} \mu_{i}$. Então, as seguintes afirmações são válidas para toda matriz $U \succeq 0$ :

(i) A seqüência $\left\{g\left(U_{k}\right)\right\}$ é não-crescente.

(ii) $\mu_{k}\left(g\left(U_{k}\right)-g(U)\right) \leq \mathrm{D}\left(U, U_{k-1}\right)-\mathrm{D}\left(U, U_{k}\right)$ para todo inteiro $k \geq 1$.

(iii) $\sigma_{s}\left(g\left(U_{s}\right)-g(U)\right) \leq \mathrm{D}\left(U, U_{0}\right)-\mathrm{D}\left(U, U_{s}\right)$ para todo inteiro $s \geq 1$.

Além disso, se $\mathrm{S}_{\mathrm{DM}}^{*} \neq \emptyset$, então

(iv) A seqüência $\left\{\mathrm{D}\left(U^{*}, U_{k}\right)\right\}$ é não-crescente para toda $U^{*} \in \mathrm{S}_{\mathrm{DM}}^{*}$;

(v) $\mathrm{D}\left(U_{k}, U_{k-1}\right) \rightarrow 0$ e $\operatorname{tr}\left(U_{k}-U_{k-1}\right) \rightarrow 0$ quando $k \rightarrow \infty$.

Demonstração.

(i) A partir Lema 3.1.1(ii), sabemos que $\mathrm{D}\left(U_{k}, U_{k-1}\right) \geq 0$ e $\mathrm{D}\left(U_{k-1}, U_{k-1}\right)=0$, para todo inteiro $k>0$. A partir da definição da seqüência $\left\{U_{k}\right\}$ feita pela expressão (3.2), temos

$$
g\left(U_{k}\right) \leq g\left(U_{k}\right)+\frac{1}{\mu_{k}} \mathrm{D}\left(U_{k}, U_{k-1}\right) \leq g\left(U_{k-1}\right)+\frac{1}{\mu_{k}} \mathrm{D}\left(U_{k-1}, U_{k-1}\right)=g\left(U_{k-1}\right),
$$

resultando que $g\left(U_{k}\right) \leq g\left(U_{k-1}\right)$.

(ii) A partir da definição de subdiferencial feita no Capítulo 1 e da expressão (3.5) do Lema 3.1.4, sabemos que

$$
g\left(U_{k}\right)+\left\langle U-U_{k}, \mu^{-1}\left(\ln U_{k-1}-\ln U_{k}\right)\right\rangle \leq g(U)
$$


para toda matriz $U \succeq 0$. A expressão acima equivale a

$$
\begin{aligned}
\mu_{k}\left(g\left(U_{k}\right)-g(U)\right) & \leq\left\langle U-U_{k}, \ln U_{k}-\ln U_{k-1}\right\rangle & & \\
& =\mathrm{D}\left(U, U_{k-1}\right)-\mathrm{D}\left(U, U_{k}\right)-\mathrm{D}\left(U_{k}, U_{k-1}\right) & & {[\text { Lema }(3.1 .3)(i i)] } \\
& \leq \mathrm{D}\left(U, U_{k-1}\right)-\mathrm{D}\left(U, U_{k}\right) & & {[\operatorname{Lema}(3.1 .3)(i i)], }
\end{aligned}
$$

implicando que $\mu_{k}\left(g\left(U_{k}\right)-g(U)\right) \leq \mathrm{D}\left(U, U_{k-1}\right)-\mathrm{D}\left(U, U_{k}\right)$, o que prova o item (ii) do lema.

(iii) Pela definição da seqüência $\left\{\sigma_{s}\right\}$ e pelo item (i), temos

$$
\sum_{k=1}^{s} \mu_{k} g\left(U_{k}\right) \geq \sum_{k=1}^{s} \mu_{k} g\left(U_{s}\right)=\sigma_{s} g\left(U_{s}\right) .
$$

Ademais, ao efetuarmos a soma das inequações do item (ii) para $k=1, \ldots, s$, temos

$$
\sum_{k=1}^{s} \mu_{k}\left(g\left(U_{k}\right)-g(U)\right) \leq \sum_{k=1}^{s}\left(\mathrm{D}\left(U, U_{k-1}\right)-\mathrm{D}\left(U, U_{k}\right)\right),
$$

que é equivalente a

$$
\sum_{k=1}^{s} \mu_{k} g\left(U_{k}\right)-\sigma_{s} g(U) \leq \mathrm{D}\left(U, U_{0}\right)-\mathrm{D}\left(U, U_{s}\right) .
$$

A partir dessa desigualdade, combinada à expressão (3.8), obtemos

$$
\sigma_{s}\left(g\left(U_{s}\right)-g(U)\right) \leq \mathrm{D}\left(U, U_{0}\right)-\mathrm{D}\left(U, U_{s}\right)
$$

conforme queríamos.

(iv) Seja $U^{*} \in \mathrm{S}_{\mathrm{DM}}^{*}$. Logo, $g\left(U^{*}\right) \leq g\left(U_{k}\right)$, para todo inteiro não negativo $k$. A partir do item (ii), obtemos

$$
0 \leq \mu_{k}\left(g\left(U_{k}\right)-g\left(U^{*}\right)\right) \leq \mathrm{D}\left(U^{*}, U_{k-1}\right)-\mathrm{D}\left(U^{*}, U_{k}\right),
$$

implicando que $\mathrm{D}\left(U^{*}, U_{k}\right) \leq \mathrm{D}\left(U^{*}, U_{k-1}\right)$.

(v) Seja $U^{*} \in \mathrm{S}_{\mathrm{DM}}^{*}$. Pelo item (iv), a seqüência $\left\{\mathrm{D}\left(U^{*}, U_{k}\right)\right\}$ é não crescente. Além disso, $\mathrm{D}\left(U^{*}, U_{k}\right) \geq 0$, a partir do Lema 3.1.1(ii). Dessa forma, a seqüência $\left\{\mathrm{D}\left(U^{*}, U_{k}\right)\right\}$ é convergente, implicando que $\mathrm{D}\left(U^{*}, U_{k-1}\right)-\mathrm{D}\left(U^{*}, U_{k}\right) \rightarrow 0$. A partir das demonstrações dos ítens (ii) e (iv), obtemos

$$
0 \leq \mu_{k}\left(g\left(U_{k}\right)-g\left(U^{*}\right)\right) \leq \mathrm{D}\left(U^{*}, U_{k-1}\right)-\mathrm{D}\left(U^{*}, U_{k}\right)-\mathrm{D}\left(U_{k}, U_{k-1}\right),
$$

de onde segue que $0 \leq \mathrm{D}\left(U_{k}, U_{k-1}\right) \leq \mathrm{D}\left(U^{*}, U_{k-1}\right)-\mathrm{D}\left(U^{*}, U_{k}\right)$. Logo, podemos concluir que $\mathrm{D}\left(U_{k}, U_{k-1}\right) \rightarrow 0$. A partir do Lema 3.1.2, sabemos que $\operatorname{tr}\left(U_{k}-U_{k-1}\right) \rightarrow 0$ também, o que completa a prova do item (v).

Após a exposição dos dois lemas anteriores, podemos agora apresentar um resultado sobre a convergência do Algoritmo (API). 
Teorema 3.1.6 ([DT99, Teorema 4.1]). Seja $\left\{U_{k}\right\}$ a seqüência gerada pelo algoritmo de ponto proximal interior abordado. Sob as Hipóteses 3.1.1, 3.1.2 e 3.1.3, é válido para todo inteiro $s \geq 0$ que

(i) para toda matriz $U \in \mathbb{S}_{+}^{m}, g\left(U_{s}\right)-g(U) \leq \sigma_{s}^{-1} \mathrm{D}\left(U, U_{0}\right)$;

(ii) se $\sigma_{s} \rightarrow \infty$, então $\lim _{s \rightarrow \infty} g\left(U_{s}\right)=g^{*}$;

(iii) se $\mathrm{S}_{\mathrm{DM}}^{*}$ é não-vazio, então a seqüência $\left\{U_{k}\right\}$ é limitada e cada um de seus pontos limites é uma solução de (DM).

Demonstração.

(i) Pelo Lema 3.1.5(iii), temos

$$
\begin{aligned}
\sigma_{s}\left(g\left(U_{s}\right)-g(U)\right) & \leq \mathrm{D}\left(U, U_{0}\right)-\mathrm{D}\left(U, U_{s}\right) \\
& \leq \mathrm{D}\left(U, U_{0}\right)
\end{aligned}
$$

Assim, segue da inequação acima que $g\left(U_{s}\right)-g(U) \leq \sigma_{s}^{-1} \mathrm{D}\left(U, U_{0}\right)$.

(ii) Ao tomarmos o limite na expressão do item (i), temos

$$
\limsup _{s \rightarrow \infty}\left(g\left(U_{s}\right)-g(U)\right) \leq \limsup _{s \rightarrow \infty} \frac{1}{\sigma_{s}} \mathrm{D}\left(U, U_{0}\right)
$$

para toda matriz $U \in \mathbb{S}_{+}^{m}$. Devido à hipótese de $\sigma_{s} \rightarrow \infty$ quando $s \rightarrow \infty$, a desigualdade acima implica que

$$
\limsup _{s \rightarrow \infty}\left(g\left(U_{s}\right)-g(U)\right) \leq 0
$$

resultando que $\limsup _{s \rightarrow \infty} g\left(U_{s}\right) \leq g(U)$, para toda matriz $U \in \mathbb{S}_{+}^{m}$. Assim, te$\operatorname{mos} \lim \sup _{s \rightarrow \infty} g\left(U_{s}\right) \leq g^{*}$. Por outro lado, $g\left(U_{s}\right) \geq g^{*}$. Portanto, concluímos que $\lim _{s \rightarrow \infty} g\left(U_{s}\right)=g^{*}$.

(iii) Suponhamos que $\mathrm{S}_{\mathrm{DM}}^{*}$ seja não vazio. Pelo Lema 3.1.1(iv), sabemos que a função $\mathrm{D}(U, \cdot)$ tem conjuntos de nível limitados para toda matriz $U \in \mathbb{S}_{+}^{m}$. Além disso, pelo Lema 3.1.5, sabemos que a seqüência $\left\{\mathrm{D}\left(U_{k}, U^{*}\right)\right\}$ é não crescente para qualquer matriz $U^{*} \in \mathrm{S}_{\mathrm{DM}}^{*}$. Ao combinarmos esses dois fatos, podemos ver que a seqüência $\left\{U_{k}\right\}$ gerada pelo Algoritmo (API) é limitada. Sejam $\bar{U} \in \mathbb{S}_{+}^{m}$ um ponto limite da dessa seqüência e $\left\{U_{k_{j}}\right\}_{j=1}^{\infty}$ uma subseqüência de $\left\{U_{k}\right\}$ que convirja para $\bar{U}$. Já que $g$ é semicontínua inferior, sabemos que $g(\bar{U}) \leq \liminf _{j \rightarrow \infty} g\left(U_{k_{j}}\right)$. Por outro lado, pelo item (ii), $g\left(U_{k_{j}}\right) \rightarrow g^{*}$. Assim, $g(\bar{U})=g^{*}$, o que completa a prova.

É importante destacar que a taxa global de convergência estabelecida no Teorema 3.1.6(i) é similar àquela obtida para métodos de ponto proximal para programação convexa no $\mathbb{R}^{n}$. Além disso, se a seqüência $\left\{U_{k}\right\}$ gerada pelo Algoritmo (API) tem um ponto limite $\bar{U}$ no interior do cone de matrizes semidefinidas positivas, isto é, se $\bar{U} \in \mathbb{S}_{++}^{m}$, então podemos assegurar a convergência global da seqüência $\left\{U_{k}\right\}$ para esta solução de (DM) devido 
ao Lema 3.1.1(i). Entretanto, não se sabe se tal restrição pode ser enfraquecida porque, enquanto para qualquer seqüência $\left\{a_{k}\right\} \subset \mathbb{R}_{++}^{m}$ tal que $\left\{a^{k}\right\} \rightarrow \bar{a} \in \mathbb{R}_{+}^{m}$, temos

$$
d\left(\bar{a}, a^{k}\right) \rightarrow 0
$$

tal propriedade não pode ser estendida para a regularização D do Algoritmo (API). E essa propriedade é a adotada nas demonstrações que garantem a convergência global do método proximal entrópico no $\mathbb{R}^{n}$. Apresentamos a seguir um exemplo para ilustrar essa situação, no qual uma seqüência $\tilde{U}_{k} \subset \mathbb{S}_{++}^{m}$ converge para uma matriz $\bar{U} \in \mathbb{S}_{+}^{m} \backslash \mathbb{S}_{++}^{m}$ e $\mathrm{D}\left(\bar{U}, \tilde{U}_{k}\right) \rightarrow \infty$.

Exemplo 3.1.1 ([DT99, Exemplo 4.1]). Sejam

$$
\tilde{U}_{k}=\frac{1}{k}\left[\begin{array}{cc}
k-1+e^{-k^{2}} & \left(1-e^{-k^{2}}\right) \sqrt{k-1} \\
\left(1-e^{-k^{2}}\right) \sqrt{k-1} & (k-1) e^{-k^{2}}+1
\end{array}\right],
$$

para todo inteiro $k>0$ e

$$
\bar{U}=\left[\begin{array}{ll}
1 & 0 \\
0 & 0
\end{array}\right]
$$

A partir da decomposição espectral de $\tilde{U}_{k}$, podemos ver que seus autovalores são 1 e $e^{-k^{2}}$ com autovetores associados

$$
\left[\begin{array}{c}
\sqrt{k^{-1}(k-1)} \\
k^{-1 / 2}
\end{array}\right] e\left[\begin{array}{c}
-k^{-1 / 2} \\
\sqrt{k^{-1}(k-1)}
\end{array}\right],
$$

respectivamente. Então, $\tilde{U}_{k}$ é definida positiva para todo inteiro $k>0$, pois seus autovalores são estritamente positivos. Também podemos perceber que $\tilde{U}_{k} \rightarrow \bar{U} \in \mathbb{S}_{+}^{m} \backslash \mathbb{S}_{++}^{m}$ quando $k \rightarrow \infty$. Calculemos agora $\mathrm{D}\left(\bar{U}, \tilde{U}_{k}\right)$. Temos

$$
\begin{aligned}
\bar{U} \ln \bar{U} & =\left[\begin{array}{ll}
0 & 0 \\
0 & 0
\end{array}\right], \\
\ln \tilde{U}_{k} & =\left[\begin{array}{cc}
-k & k \sqrt{k-1} \\
k \sqrt{k-1} & -k(k-1)
\end{array}\right], \\
\bar{U} \ln \tilde{U}_{k} & =\left[\begin{array}{cc}
-k & k \sqrt{k-1} \\
0 & 0
\end{array}\right] .
\end{aligned}
$$

Logo, a partir da definição da regularização $\mathrm{D}$, temos

$$
\begin{aligned}
\mathrm{D}\left(\bar{U}, \tilde{U}_{k}\right) & =\operatorname{tr}\left(\bar{U} \ln \bar{U}-\bar{U} \ln \tilde{U}_{k}+\tilde{U}_{k}-\bar{U}\right) \\
& =\operatorname{tr}\left(\bar{U} \ln \bar{U}-\bar{U} \ln \tilde{U}_{k}\right)+\operatorname{tr}\left(\tilde{U}_{k}-\bar{U}\right) \\
& =\operatorname{tr}\left(\left[\begin{array}{cc}
k & -k \sqrt{k-1} \\
0 & 0
\end{array}\right]\right)+\operatorname{tr}\left(\tilde{U}_{k}-\bar{U}\right) \\
& =k+\operatorname{tr}\left(\tilde{U}_{k}-\bar{U}\right) .
\end{aligned}
$$

Já que $\tilde{U}_{k} \rightarrow \bar{U}$, segue que $\mathrm{D}\left(\bar{U}, \tilde{U}_{k}\right) \rightarrow \infty$ quando $k \rightarrow \infty$. 


\subsection{Método de multiplicadores exponenciais para programa- ção semidefinida}

Conforme vimos no início deste capítulo, o algoritmo proximal entrópico interior (API) pode ser usado para resolver o problema dual. E a partir da teoria de métodos de ponto proximal para programação convexa, esse algoritmo nos fornece fundamentos para o desenvolvimento de métodos de multiplicadores para resolver o problema primal também. Nesta seção, mostramos que a seqüência gerada pelo Algoritmo (API) aplicado sobre o problema dual linear é uma das seqüências fornecidas por um método de multiplicadores exponenciais que usa somente dados do problema primal associado. Além disso, sob algumas hipóteses, mostramos que os pontos limites de uma seqüência produzida por tal método de multiplicadores exponenciais são soluções do problema primal linear.

Supomos que as seguintes hipóteses adicionais são válidas para o problema primal linear:

Hipótese 3.2.1. O conjunto de soluções do problema primal linear é não-vazio e limitado.

Hipótese 3.2.2. A condição de Slater é válida, ou seja, existe $\tilde{x} \in \mathbb{R}^{n}$ tal que $A_{0}+\mathcal{A}(\tilde{x}) \prec 0$.

Sob as Hipóteses 3.2.1 e 3.2.2, sabemos, pelo Teorema 2.3.4, que a folga de dualidade é zero e que o conjunto de soluções do problema dual linear, $\mathrm{S}_{\mathrm{DL}}^{*}$, é não-vazio e compacto. Equivalentemente, o valor ótimo do problema dual é finito e $\mathcal{K}_{\mathrm{D}}(\hat{U})>-\infty$ para alguma matriz $\hat{U} \in \mathbb{S}_{+}^{m}$. Assim, ao tomarmos a função objetivo de (DM) como aquela definida pela equação (3.1), sabemos que as Hipóteses 3.1.1 e 3.1.2 são satisfeitas.

Com base no método de multiplicadores exponenciais para problemas convexos no $\mathbb{R}^{n}$ [Ber99, Seção 4.2.5], o seguinte algoritmo de multiplicadores (AME) foi apresentado em [DT99] para resolver (PSDL):

Algoritmo 3.2. (AME)

(i) Escolha uma matriz $U_{0} \in \mathbb{S}_{++}^{m}$, um parâmetro real $\bar{\mu}>0$ e uma seqüência $\left\{\mu_{k}\right\} \subseteq \mathbb{R}_{++}$ tal que $\mu_{k} \geq \bar{\mu}$ para todo $k \geq 0$.

(ii) Gere as seqüências $\left\{x^{k}\right\} \subseteq \mathbb{R}^{n} e\left\{U_{k}\right\} \subseteq \mathbb{S}_{++}^{m}$ definidas por

$$
\begin{aligned}
& x^{k+1}=\underset{x \in \mathbb{R}^{n}}{\operatorname{argmin}}\left\{c^{T} x+\frac{1}{\mu_{k}} \operatorname{tr}\left(e^{\mu_{k}\left(A_{0}+\mathcal{A}(x)\right)+\ln U_{k}}\right)\right\}, \\
& U_{k+1}=e^{\mu_{k}\left(A_{0}+\mathcal{A}\left(x^{k+1}\right)\right)+\ln U_{k}} .
\end{aligned}
$$

Exibimos a seguir um resultado que assegura que a seqüência $\left\{x^{k}\right\}$ gerada pelo método está bem definida. Para cada $\mu \in \mathbb{R}_{++}$e $B \in \mathbb{S}^{m}$ fixos, definimos $F_{(\mu, B)}: \mathbb{R}^{n} \rightarrow \mathbb{R}$ por

$$
F_{(\mu, B)}(x) \stackrel{\text { def }}{=} c^{T} x+\frac{1}{\mu} \operatorname{tr}\left(e^{\mu\left(A_{0}+\mathcal{A}(x)\right)+B}\right) .
$$

Pelo Lema 1.5.9, essa função é estritamente convexa, pois é soma de uma função linear e de uma função estritamente convexa. Ademais, indicamos por $F_{(\mu, B)}^{*} \subseteq \mathbb{R}^{n}$ seu conjunto de minimizadores. Abordamos agora quando podemos garantir que esse conjunto é não-vazio e limitado. 
Lema 3.2.1 ([DT99, Lema 5.1]). Se a Hipótese 3.2.1 é válida, então $F_{(\mu, B)}^{*}$ é não-vazio e limitado para todo escalar $\mu>0$ e toda matriz $B \in \mathbb{S}^{m}$.

\section{Demonstração.}

Primeiramente, provemos que $F_{(\mu, B)}$ é coerciva. A prova será feita por contradição. Suponha que $F_{(\mu, B)}$ não é coerciva, ou seja, que algum conjunto de nível de $F_{(\mu, B)}$ não seja limitado. Assim, existem uma seqüência $\left\{\hat{x}^{k}\right\} \subset \mathbb{R}^{n}$ e um escalar $\delta$ tal que

$$
\begin{array}{r}
\left\|\hat{x}^{k}\right\| \underset{k \rightarrow \infty}{\longrightarrow} \infty, \\
\lim _{k \rightarrow \infty} \frac{\hat{x}^{k}}{\left\|\hat{x}^{k}\right\|}=d \neq 0, \\
F_{(\mu, B)}\left(\hat{x}^{k}\right) \leq \delta .
\end{array}
$$

Já que $\operatorname{tr}\left(e^{U}\right)>0$ para toda matriz $U \in \mathbb{S}^{m}$, em particular, temos $\operatorname{tr}\left(e^{\mu\left(A_{0}+\mathcal{A}(x)\right)+B}\right)>0$. Então, $F_{(\mu, B)}\left(\hat{x}^{k}\right) \leq \delta$ implica que

$$
\begin{gathered}
c^{T} \hat{x}^{k}<\delta \\
0<\operatorname{tr}\left(e^{\mu\left(A_{0}+\mathcal{A}(x)\right)+B}\right)=\sum_{i=1}^{m} e^{\lambda_{i}\left(\mu\left(A_{0}+\mathcal{A}\left(\hat{x}^{k}\right)\right)+B\right)} \leq \mu\left(\delta-c^{T} \hat{x}^{k}\right)
\end{gathered}
$$

Ao dividirmos a inequação (3.14) por $\left\|\hat{x}^{k}\right\|$ e tomarmos o limite para $k \rightarrow \infty$, obtemos $c^{T} d \leq 0$ a partir da expressão (3.12). Por outro lado, pela expressão (3.15), sabemos que

$$
0<e^{\lambda_{i}\left(\mu\left(A_{0}+\mathcal{A}\left(\hat{x}^{k}\right)\right)+B\right)} \leq \mu\left(\delta-c^{T} \hat{x}^{k}\right), \quad \text { para } i=1, \ldots, m .
$$

Ao tomarmos o logaritmo em ambos os lados da segunda desigualdade acima e dividirmos por $\left\|\hat{x}^{k}\right\|$, obtemos

$$
\frac{\lambda_{i}\left(\mu\left(A_{0}+\mathcal{A}\left(\hat{x}^{k}\right)\right)+B\right)}{\left\|\hat{x}^{k}\right\|} \leq \frac{\ln \left(\mu\left(\delta-c^{T} \hat{x}^{k}\right)\right)}{\left\|\hat{x}^{k}\right\|}
$$

para $i=1, \ldots, m$. Já que $\lambda_{i}(\cdot)$ é uma função homogênea e $\mathcal{A}$ é um operador linear, a inequação acima implica que

$$
\begin{aligned}
\frac{\lambda_{i}\left(\mu\left(A_{0}+\mathcal{A}\left(\hat{x}^{k}\right)\right)+B\right)}{\left\|\hat{x}^{k}\right\|} & =\lambda_{i}\left(\mu\left(\frac{1}{\left\|\hat{x}^{k}\right\|} A_{0}+\mathcal{A}\left(\frac{\hat{x}^{k}}{\left\|\hat{x}^{k}\right\|}\right)\right)+\frac{1}{\left\|\hat{x}^{k}\right\|} B\right) \\
& =\mu \lambda_{i}\left(\frac{1}{\left\|\hat{x}^{k}\right\|} A_{0}+\mathcal{A}\left(\frac{\hat{x}^{k}}{\left\|\hat{x}^{k}\right\|}\right)+\frac{1}{\mu\left\|\hat{x}^{k}\right\|} B\right) \\
& \leq \frac{\ln \left(\mu\left(\delta-c^{T} \hat{x}^{k}\right)\right)}{\left\|\hat{x}^{k}\right\|}
\end{aligned}
$$

para $i=1, \ldots, m$. Ao tomarmos o limite para $k \rightarrow \infty$, obtemos $\lambda_{i}(\mathcal{A}(d)) \leq 0$, para $i=1, \ldots, m$, o que equivale a $\mathcal{A}(d) \preceq 0$. Logo, para $x^{*} \in \mathrm{S}_{\mathrm{P}}^{*}$ e $\alpha \geq 0$, sabemos que

$$
A_{0}+\mathcal{A}\left(x^{*}+\alpha d\right)=\underbrace{A_{0}+\mathcal{A}\left(x^{*}\right)}_{\preceq 0}+\alpha \mathcal{A}(d) \preceq 0,
$$


resultando que $x^{*}+\alpha d$ é um ponto viável de (PSDL) para todo $\alpha \geq 0$. Já provamos que $c^{T} d \leq 0$. Dessa forma, se $c^{T} d=0$, temos $c^{T}\left(x^{*}+\alpha d\right)=c^{T} x^{*}=\mathrm{f}_{*}^{\mathrm{P}}$ e podemos concluir que $x^{*}+\alpha d \in \mathrm{S}_{\mathrm{P}}^{*}$ para todo $\alpha \geq 0$, o que contraria a hipótese de $\mathrm{S}_{\mathrm{P}}^{*}$ ser limitado. Por outro lado, se $c^{T} d<0$, temos $c^{T}\left(x^{*}+\alpha d\right)=c^{T} x^{*}+\alpha c^{T} d<\mathrm{f}_{*}^{\mathrm{P}}$ se $\alpha>0$, o que contraria a hipótese de $x^{*}$ ser uma solução do problema primal linear. Portanto, $F_{(\mu, B)}$ é coerciva, resultando que $F_{(\mu, B)}^{*}$ é não-vazio e limitado para todo $\mu>0$ e toda matriz $B \in \mathbb{S}^{m}$ [Ber03, Proposição 2.1.1].

A partir desse lema e do fato de $F_{(\mu, B)}$ ser estritamente convexa, sabemos que o algoritmo de multiplicadores exponenciais proposto está bem definido sob a Hipótese 3.2.1. Mostramos agora que a seqüência $\left\{U_{k}\right\}$ gerada por (AME) é a mesma seqüência gerada pelo Algoritmo (API) quando aplicado ao problema dual linear.

Lema 3.2.2 ([DT99, Lema 5.2]). A seqüência $\left\{U_{k}\right\}$ gerada pelo algoritmo de multiplicadores exponenciais (3.9a)-(3.9b) pode ser obtida através da iteração

$$
U_{k+1}=\underset{U \succeq 0}{\operatorname{argmax}}\left\{\mathcal{K}_{\mathrm{D}}(U)-\frac{1}{\mu_{k}} \mathrm{D}\left(U, U_{k}\right)\right\} .
$$

Demonstração. Primeiramente, mostremos que $A_{0}+\mathcal{A}\left(x^{k}\right) \in \partial \mathcal{K}_{\mathrm{D}}\left(U_{k+1}\right)$. Ao escrevermos uma condição de otimalidade necessária e suficiente para que o ponto $x^{k+1}$ seja um minimizador do problema definido pela equação (3.9a), temos

$$
\begin{array}{rlrl}
0 & =\left.\frac{\partial}{\partial x_{i}}\left(c^{T} x+\frac{1}{\mu_{k}} \operatorname{tr}\left(e^{\mu_{k}\left(A_{0}+\mathcal{A}(x)\right)+\ln U_{k}}\right)\right)\right|_{x=x^{k+1}} \\
& =c_{i}+\operatorname{tr}\left(A_{i} e^{\mu_{k}\left(A_{0}+\mathcal{A}\left(x^{k+1}\right)\right)+\ln U_{k}}\right) & \\
& =c_{i}+\operatorname{tr}\left(A_{i} U_{k+1}\right) & & \text { [expressão }(3.9 \mathrm{~b})] \\
& =c_{i}+\left\langle A_{i}, U_{k+1}\right\rangle &
\end{array}
$$

para $i=1, \ldots, n$. Logo, $c+\mathcal{A}^{*}\left(U_{k+1}\right)=0$, implicando que $\nabla_{x} \mathrm{~L}\left(x^{k+1}, U_{k+1}\right)=0$. Devido à função lagrangiana $\mathrm{L}$ ser convexa em relação ao primeiro argumento, segue-se que $x^{k+1}$ é um minimizador de $\mathrm{L}\left(\cdot, U_{k+1}\right)$. Assim, $\mathcal{K}_{\mathrm{D}}\left(U_{k+1}\right)=\mathrm{L}\left(x^{k+1}, U_{k+1}\right)$. Para toda matriz $U \in \mathbb{S}^{m}$, temos

$$
\begin{aligned}
\mathcal{K}_{\mathrm{D}}(U) & =\inf _{x \in \mathbb{R}^{n}} \mathrm{~L}(x, U) \\
& =\inf _{x \in \mathbb{R}^{n}}\left\{c^{T} x+\left\langle U, A_{0}+\mathcal{A}(x)\right\rangle\right\} \\
& \leq c^{T} x^{k+1}+\left\langle U, A_{0}+\mathcal{A}\left(x^{k+1}\right)\right\rangle \\
& =c^{T} x^{k+1}+\left\langle U_{k+1}, A_{0}+\mathcal{A}\left(x^{k+1}\right)\right\rangle+\left\langle U-U_{k+1}, A_{0}+\mathcal{A}\left(x^{k+1}\right)\right\rangle \\
& =\mathrm{L}\left(x^{k+1}, U_{k+1}\right)+\left\langle U-U_{k+1}, A_{0}+\mathcal{A}\left(x^{k+1}\right)\right\rangle \\
& =\mathcal{K}_{\mathrm{D}}\left(U_{k+1}\right)+\left\langle U-U_{k+1}, A_{0}+\mathcal{A}\left(x^{k+1}\right)\right\rangle,
\end{aligned}
$$


resultando que $\mathcal{K}_{\mathrm{D}}(U) \leq \mathcal{K}_{\mathrm{D}}\left(U_{k+1}\right)+\left\langle U-U_{k+1}, A_{0}+\mathcal{A}\left(x^{k+1}\right)\right\rangle$. Logo, $A_{0}+\mathcal{A}\left(x^{k+1}\right) \in$ $\partial \mathcal{K}_{\mathrm{D}}\left(U_{k+1}\right)$ porque a função $\mathcal{K}_{\mathrm{D}}$ é côncava. Ao tomarmos o logaritmo nos dois lados da expressão $(3.9 \mathrm{~b})$, obtemos

$$
\ln U_{k+1}-\ln U_{k}=\mu_{k}\left(A_{0}+\mathcal{A}\left(x^{k+1}\right)\right),
$$

implicando que

$$
\frac{1}{\mu}\left(\ln U_{k}-\ln U_{k+1}\right) \in \partial \mathcal{K}_{\mathrm{D}}\left(U_{k+1}\right) .
$$

Essa relação é a condição de otimalidade dada pelo Lema 3.1.4 para que a matriz $U_{k+1}$ seja a solução única do problema

$$
\min _{U \succeq 0}\left\{-\mathcal{K}_{\mathrm{D}}(U)+\frac{1}{\mu_{k}} \mathrm{D}\left(U, U_{k}\right)\right\}
$$

o qual possui o mesmo conjunto de soluções que o problema definido por (3.16). Assim, nós concluímos a demonstração.

É importante ressaltar que, pelos Lemas 3.2 .1 e 3.2.2, sabemos que as matrizes $U_{k}$ geradas pelo Algoritmo (AME) são definidas positivas e são pontos viáveis do problema dual linear, para todo $k \geq 1$. Assim, a Hipótese 3.1 .3 pedida na análise de convergência do Algoritmo (API) também é satisfeita. Depois de termos provado esses lemas, podemos apresentar agora o seguinte teorema sobre a convergência do Algoritmo (AME) proposto. Tal teorema estende resultados conhecidos sobre o método de multiplicadores exponenciais para programação convexa no $\mathbb{R}^{n}$.

Teorema 3.2.3 ([DT99, Teorema 5.1]). Consideremos as seqüências $\left\{x^{k}\right\}$ e $\left\{U_{k}\right\}$ geradas pelo algoritmo de multiplicadores exponenciais (AME) em (3.9a) e (3.9b), respectivamente. Sejam

$$
x^{*} \in \mathrm{S}_{\mathrm{P}}^{*}, \quad \nu_{k}=\sum_{i=1}^{k} \mu_{k}, \quad \bar{x}^{k}=\sum_{i=1}^{k} \frac{\mu_{i}}{\nu_{k}} x^{i} .
$$

Sob as Hipóteses 3.2.1 e 3.2.2, os seguintes resultados são válidos:

(a) A seqüência $\left\{U_{k}\right\} \subseteq \mathbb{S}_{++}^{m}$ é limitada e todos os seus pontos limites são soluções do problema dual.

(b) $\left\langle U_{k}, A_{0}+\mathcal{A}\left(x^{k}\right)\right\rangle \rightarrow 0$ quando $k \rightarrow \infty$.

(c) $\limsup _{k \rightarrow \infty} \lambda_{\max }\left(A_{0}+\mathcal{A}\left(\bar{x}^{k}\right)\right) \leq 0$.

(d) Para $k \rightarrow \infty$, obtemos que $c^{T} x^{k} \rightarrow \mathrm{f}_{*}^{\mathrm{D}}$ e, como resultado, $c^{T} \bar{x}^{k} \rightarrow \mathrm{f}_{*}^{\mathrm{D}}$ também.

(e) A seqüência $\left\{\bar{x}^{k}\right\}$ é limitada.

(f) Todo ponto limite de $\left\{\bar{x}^{k}\right\}$ é uma solução do problema primal. 
(g) $\lim _{k \rightarrow \infty} c^{T} x^{k}=\lim _{k \rightarrow \infty} \mathcal{K}_{\mathrm{D}}\left(U_{k}\right)=\mathrm{f}_{*}^{\mathrm{P}}$.

Demonstração.

(a) A partir do Lema 3.2.2, sabemos que a seqüência $\left\{U_{k}\right\}$ gerada pelo Algoritmo (AME) é a mesma produzida pelo Algoritmo (API) aplicado ao problema dual. Sob a Hipótese 3.2.1, o valor ótimo do problema primal linear é finito. Logo, já que a Hipótese 3.2.2 também vale, obtemos que o conjunto de soluções do problema dual é não-vazio e compacto pelo Teorema 2.3.4. Assim, pelo Teorema 3.1.6(iii), vemos que a seqüência $\left\{U_{k}\right\}$ é limitada e todos os seus pontos limites são soluções do problema dual.

(b) Ao tomarmos o logaritmo nos dois lados da equação (3.9b), temos

$$
\mu_{k}\left(A_{0}+\mathcal{A}\left(x^{k+1}\right)\right)=\ln U_{k+1}-\ln U_{k} .
$$

Logo, pela definição da regularização $\mathrm{D}$, sabemos que

$$
\begin{aligned}
\mathrm{D}\left(U_{k+1}, U_{k}\right) & =\operatorname{tr}\left(U_{k+1} \ln U_{k+1}-U_{k+1} \ln U_{k}+U_{k}-U_{k+1}\right) \\
& =\operatorname{tr}\left(U_{k+1}\left(\ln U_{k+1}-\ln U_{k}\right)+U_{k}-U_{k+1}\right) \\
& =\operatorname{tr}\left(\mu_{k} U_{k+1}\left(A_{0}+\mathcal{A}\left(x^{k+1}\right)\right)+U_{k}-U_{k+1}\right) .
\end{aligned}
$$

Pela nossa escolha do produto interno associado a $\mathbb{S}^{m}$ e pela propriedade do traço ser uma função linear, obtemos

$$
\begin{aligned}
\mathrm{D}\left(U_{k+1}, U_{k}\right) & =\operatorname{tr}\left(\mu_{k} U_{k+1}\left(A_{0}+\mathcal{A}\left(x^{k+1}\right)\right)\right)+\operatorname{tr}\left(U_{k}-U_{k+1}\right) \\
& =\mu_{k}\left\langle U_{k+1}, A_{0}+\mathcal{A}\left(x^{k+1}\right)\right\rangle+\operatorname{tr}\left(U_{k}-U_{k+1}\right) .
\end{aligned}
$$

Já que, devido ao Lema 3.1.5(v), $\mathrm{D}\left(U_{k+1}, U_{k}\right) \rightarrow 0$ e $\operatorname{tr}\left(U_{k}-U_{k+1}\right) \rightarrow 0$ quando $k \rightarrow \infty$ e a seqüência $\left\{\mu_{k}\right\}$ é limitada inferiormente por um real estritamente positivo, segue-se que $\left\langle U_{k+1}, A_{0}+\mathcal{A}\left(x^{k+1}\right)\right\rangle \rightarrow 0$.

(c) Já que o mapa $A_{0}+\mathcal{A}(x)$ é afim, nós temos

$$
\begin{aligned}
A_{0}+\mathcal{A}\left(\bar{x}^{k}\right) & =A_{0}+\mathcal{A}\left(\sum_{i=1}^{k} \frac{\mu_{i}}{\nu_{k}} x^{i}\right) \\
& =\frac{\nu_{k}}{\nu_{k}} A_{0}+\frac{1}{\nu_{k}} \sum_{i=1}^{k} \mu_{i} \mathcal{A}\left(x^{i}\right) \\
& =\frac{1}{\nu_{k}} \sum_{i=1}^{k} \mu_{i}\left(A_{0}+\mathcal{A}\left(x^{i}\right)\right) \\
& =\frac{1}{\nu_{k}} \sum_{i=1}^{k}\left(\ln U_{i}-\ln U_{i-1}\right) \\
& =\frac{1}{\nu_{k}}\left(\ln U_{k}-\ln U_{0}\right) .
\end{aligned}
$$


Então, por um corolário do Teorema de Weyl (Corolário 1.2.6), sabemos que

$$
\begin{aligned}
\lambda_{\max }\left(A_{0}+\mathcal{A}\left(\bar{x}^{k}\right)\right) & \leq \lambda_{\max }\left(\frac{1}{\nu_{k}} \ln U_{k}\right)+\lambda_{\max }\left(-\frac{1}{\nu_{k}} \ln U_{0}\right) \\
& =\frac{1}{\nu_{k}} \lambda_{\max }\left(\ln U_{k}\right)+\frac{1}{\nu_{k}} \lambda_{\max }\left(-\ln U_{0}\right) \\
& =\frac{1}{\nu_{k}} \lambda_{\max }\left(\ln U_{k}\right)+\frac{1}{\nu_{k}} \lambda_{\max }\left(\ln U_{0}^{-1}\right),
\end{aligned}
$$

implicando que

$$
\limsup _{k \rightarrow \infty} \lambda_{\max }\left(A_{0}+\mathcal{A}\left(\bar{x}^{k}\right)\right) \leq \limsup _{k \rightarrow \infty} \frac{1}{\nu_{k}} \lambda_{\max }\left(\ln U_{k}\right)+\limsup _{k \rightarrow \infty} \frac{1}{\nu_{k}} \lambda_{\max }\left(\ln U_{0}^{-1}\right) .
$$

Já que a seqüência $\left\{\mu_{k}\right\}$ é limitada inferiormente por um real estritamente positivo, temos $\nu_{k} \rightarrow \infty$. Dessa forma, $\nu_{k}{ }^{-1} \lambda_{\max }\left(\ln U_{0}^{-1}\right) \rightarrow 0$. A partir da definição de função matricial, também temos

$$
\limsup _{k \rightarrow \infty} \frac{1}{\nu_{k}} \lambda_{\max }\left(\ln U_{k}\right)=\limsup _{k \rightarrow \infty} \frac{1}{\nu_{k}} \ln \left(\lambda_{\max }\left(U_{k}\right)\right) .
$$

Pelo item (a), a seqüência $\left\{U_{k}\right\}$ é limitada, resultando que a seqüência $\left\{\lambda_{\max }\left(U_{k}\right)\right\}$

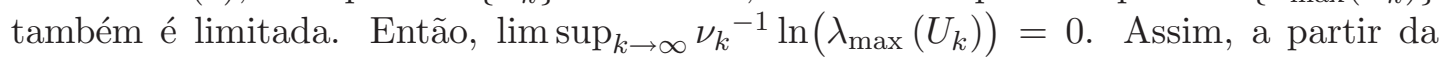
desigualdade (3.17), obtemos

$$
\limsup _{k \rightarrow \infty} \lambda_{\max }\left(A_{0}+\mathcal{A}\left(\bar{x}^{k}\right)\right) \leq 0 .
$$

(d) Segundo a demonstração do Lema 3.2.2, sabemos que as matrizes $U_{k}$ são viáveis para (DSDL), para todo inteiro $k>0$. Assim,

$$
\begin{aligned}
\left\langle U_{k}, A_{0}+\mathcal{A}\left(x^{k}\right)\right\rangle & =\left\langle U_{k}, A_{0}\right\rangle+\left\langle U_{k}, \mathcal{A}\left(x^{k}\right)\right\rangle & & \\
& =\left\langle U_{k}, A_{0}\right\rangle+\left\langle\mathcal{A}^{*}\left(U_{k}\right), x^{k}\right\rangle & & \\
& =\left\langle U_{k}, A_{0}\right\rangle-c^{T} x^{k} & & \text { [viabilidade de } U_{k} \text { ] } \\
& =\mathcal{K}_{\mathrm{D}}\left(U_{k}\right)-c^{T} x^{k} . & & \text { [equação (2.2)] }
\end{aligned}
$$

Pelo item (b), nós sabemos que $\left\langle U_{k}, A_{0}+\mathcal{A}\left(x^{k}\right)\right\rangle \rightarrow 0$ quando $k \rightarrow \infty$. Por outro lado, pelo Teorema 3.1.6(ii), obtemos $\lim _{k \rightarrow \infty} \mathcal{K}_{\mathrm{D}}\left(U_{k}\right)=\mathrm{f}_{*}^{\mathrm{D}}$. Logo, $c^{T} x^{k} \rightarrow \mathrm{f}_{*}^{\mathrm{D}}$ para $k \rightarrow \infty$. A partir da definição da seqüência $\left\{\bar{x}^{k}\right\}$, obtemos

$$
c^{T} \bar{x}^{k}=c^{T}\left(\sum_{i=1}^{k} \frac{\mu_{i}}{\nu_{k}} x^{i}\right)=c^{T}\left(\sum_{i=1}^{k} \frac{\mu_{i}}{\sum_{j=1}^{k} \mu_{j}} x^{i}\right)=\frac{\sum_{i=1}^{k} \mu_{i} c^{T} x^{i}}{\sum_{i=1}^{k} \mu_{i}},
$$

implicando, pelo Teorema A.2, que $c^{T} \bar{x}^{k} \rightarrow \mathrm{f}_{*}^{\mathrm{D}}$ também. 
(e) Suponhamos, por contradição, que a seqüência $\left\{\bar{x}^{k}\right\}$ seja ilimitada. Pela Hipótese 3.2.1, o conjunto de soluções de (PSDL) é limitado. Sejam $\tilde{x}$ um elemento de $\mathrm{S}_{\mathrm{P}}^{*}$ de norma máxima e $\alpha_{k} \stackrel{\text { def }}{=} 1-3\|\tilde{x}\| /\left(\left\|\bar{x}^{k}-\tilde{x}\right\|\right)$. Já que $\left\|\bar{x}^{k}\right\| \rightarrow \infty$, sabemos que existe $k_{0}$ tal que $0<\alpha_{k}<1$, para todo $k \geq k_{0}$. Seja $z^{k} \stackrel{\text { def }}{=} \alpha_{k} \tilde{x}+\left(1-\alpha_{k}\right) \bar{x}^{k}$. Pela definição de norma, temos

$$
\left\|z^{k}\right\|=\left\|\alpha_{k} \tilde{x}+\left(1-\alpha_{k}\right) \bar{x}^{k}\right\| \leq\left|\alpha_{k}\right|\|\tilde{x}\|+\left|1-\alpha_{k}\right|\left\|\bar{x}^{k}\right\| .
$$

Assim, para todo $k \geq k_{0}$,

$$
\begin{aligned}
\left\|z^{k}\right\| & \leq \alpha_{k}\|\tilde{x}\|+\left(1-\alpha_{k}\right)\left\|\bar{x}^{k}\right\| \\
& =\alpha_{k}\left(\|\tilde{x}\|-\left\|\bar{x}^{k}\right\|\right)+\left\|\bar{x}^{k}\right\| \\
& =\left(1-\frac{3\|\tilde{x}\|}{\left\|\bar{x}^{k}-\tilde{x}\right\|}\right)\left(\|\tilde{x}\|-\left\|\bar{x}^{k}\right\|\right)+\left\|\bar{x}^{k}\right\| \\
& =\|\tilde{x}\|+\frac{3\|\tilde{x}\|}{\left\|\bar{x}^{k}-\tilde{x}\right\|}\left(\left\|\bar{x}^{k}\right\|-\|\tilde{x}\|\right) \\
& \leq\|\tilde{x}\|+3\|\tilde{x}\| \\
& =4\|\tilde{x}\| .
\end{aligned}
$$

Por outro lado, novamente pela definição de norma, segue que

$$
\left\|\left(1-\alpha_{k}\right) \bar{x}^{k}\right\|=\left\|z^{k}-\alpha_{k} \tilde{x}\right\| \leq\left\|z^{k}\right\|+\left|\alpha_{k}\right|\|\tilde{x}\|
$$

Assim, para todo $k \geq k_{0}$,

$$
\begin{aligned}
& \left(1-\alpha_{k}\right)\left\|\bar{x}^{k}\right\| \leq\left\|z^{k}\right\|+\alpha_{k}\|\tilde{x}\| \\
& \Rightarrow\left(1-\alpha_{k}\right)\left\|\bar{x}^{k}\right\|-\alpha_{k}\|\tilde{x}\| \leq\left\|z^{k}\right\| \\
& \Rightarrow\left\|\bar{x}^{k}\right\|-\alpha_{k}\left(\left\|\bar{x}^{k}\right\|+\|\tilde{x}\|\right) \leq\left\|z^{k}\right\| \\
& \Rightarrow\left\|\bar{x}^{k}\right\|-\left(1-\frac{3\|\tilde{x}\|}{\left\|\bar{x}^{k}-\tilde{x}\right\|}\right)\left(\left\|\bar{x}^{k}\right\|+\|\tilde{x}\|\right) \leq\left\|z^{k}\right\| \\
& \Rightarrow-\|\tilde{x}\|+\frac{3\|\tilde{x}\|}{\left\|\bar{x}^{k}-\tilde{x}\right\|}\left(\left\|\bar{x}^{k}\right\|+\|\tilde{x}\|\right) \leq\left\|z^{k}\right\| \\
& \Rightarrow-\|\tilde{x}\|+\frac{3\|\tilde{x}\|}{\left\|\bar{x}^{k}\right\|+\|\tilde{x}\|}\left(\left\|\bar{x}^{k}\right\|+\|\tilde{x}\|\right) \leq\left\|z^{k}\right\| \\
& \Rightarrow 2\|\tilde{x}\| \leq\left\|z^{k}\right\| .
\end{aligned}
$$

Ao combinarmos as expressões (3.18) e (3.19), vemos que $2\|\tilde{x}\| \leq\left\|z^{k}\right\| \leq 4\|\tilde{x}\|$, para todo $k \geq k_{0}$. Logo, a seqüência $\left\{z^{k}\right\}$ é limitada. Seja $\bar{z}$ um ponto limite dessa seqüência. Já que

$$
\begin{aligned}
A_{0}+\mathcal{A}\left(z^{k}\right) & =A_{0}+\mathcal{A}\left(\alpha_{k} \tilde{x}+\left(1-\alpha_{k}\right) \bar{x}^{k}\right) \\
& =\alpha_{k}\left(A_{0}+\mathcal{A}(\tilde{x})\right)+\left(1-\alpha_{k}\right)\left(A_{0}+\mathcal{A}\left(\bar{x}^{k}\right)\right),
\end{aligned}
$$

$\alpha_{k} \rightarrow 1$ para $k \rightarrow \infty$ e, pelo item (c), $\lim \sup _{k \rightarrow \infty} \lambda_{\max }\left(A_{0}+\mathcal{A}\left(\bar{x}^{k}\right)\right) \leq 0$, temos que $A_{0}+\mathcal{A}(\bar{z}) \preceq 0$, isto é, $\bar{z}$ é um ponto viável do problema primal. Então, $c^{T} \tilde{x} \leq c^{T} \bar{z}$. Por 
outro lado, pelo item $(\mathrm{d})$, sabemos que $c^{T} \bar{x}^{k} \rightarrow \mathrm{f}_{*}^{\mathrm{D}}$. E pelo Corolário 2.2.2, obtemos $\mathrm{f}_{*}^{\mathrm{D}} \leq c^{T} \tilde{x}$, implicando que, para todo $\epsilon>0$, existe um inteiro $k_{\epsilon}>0$ tal que

$$
c^{T} \bar{x}^{k} \leq c^{T} \tilde{x}+\epsilon
$$

para todo $k \geq k_{\epsilon}$. Assim, para todo $\epsilon>0$, existe um inteiro estritamente positivo $k_{\epsilon}$ tal que para todo $k \geq \max \left\{k_{\epsilon}, k_{0}\right\}$, temos

$$
\begin{aligned}
c^{T} z^{k} & =\alpha_{k} c^{T} \tilde{x}+\left(1-\alpha_{k}\right) c^{T} \bar{x}^{k} \\
& \leq \alpha_{k} c^{T} \tilde{x}+\left(1-\alpha_{k}\right)\left(c^{T} \tilde{x}+\epsilon\right) \\
& =c^{T} \tilde{x}+\left(1-\alpha_{k}\right) \epsilon,
\end{aligned}
$$

resultando que $c^{T} z^{k} \leq c^{T} \tilde{x}+\left(1-\alpha_{k}\right) \epsilon$. Logo, devido a $\alpha_{k} \rightarrow 1$, obtemos

$$
\limsup _{k \rightarrow \infty} c^{T} z^{k} \leq \limsup _{k \rightarrow \infty}\left(c^{T} \tilde{x}+\left(1-\alpha_{k}\right) \epsilon\right)=c^{T} \tilde{x} .
$$

Em particular, sabemos que $c^{T} \bar{z} \leq c^{T} \tilde{x}$. Já que $\bar{z}$ é um ponto viável de (PSDL), então esse ponto também é uma solução do problema primal e $\|\bar{z}\|>\|\tilde{x}\|$, o que contradiz a nossa de escolha de $\tilde{x}$. Portanto, a seqüência $\left\{\bar{x}^{k}\right\}$ é limitada.

(f) Seja $\bar{x}$ um ponto limite da seqüência $\left\{\bar{x}^{k}\right\}$. Já que, pelo item (c),

$$
\limsup _{k \rightarrow \infty} \lambda_{\max }\left(A_{0}+\mathcal{A}\left(\bar{x}^{k}\right)\right) \leq 0
$$

sabemos que $A_{0}+\mathcal{A}(\bar{x}) \preceq 0$, ou seja, $\bar{x}$ é um ponto viável do problema primal. Então, $c^{T} \bar{x} \geq \mathrm{f}_{*}^{\mathrm{P}}$. Por outro lado, pelo item (d), $c^{T} \bar{x}^{k} \rightarrow \mathrm{f}_{*}^{\mathrm{D}}$. Logo, $c^{T} \bar{x}=\mathrm{f}_{*}^{\mathrm{D}}$. Novamente pelo Corolário 2.2.2, obtemos $c^{T} \bar{x}=\mathrm{f}_{*}^{\mathrm{P}}$.

(g) A partir da demonstração do item (d), sabemos que

$$
\lim _{k \rightarrow \infty} c^{T} x^{k}=\lim _{k \rightarrow \infty} \mathcal{K}_{\mathrm{D}}\left(U_{k}\right)=\mathrm{f}_{*}^{\mathrm{D}} .
$$

Já que, por hipótese, a folga de dualidade é zero, então $\lim _{k \rightarrow \infty} c^{T} x^{k}=\mathrm{f}_{*}^{\mathrm{D}}=\mathrm{f}_{*}^{\mathrm{P}}$, o que encerra a prova do teorema.

É importante destacar que, diferentemente de resultados existentes em programação convexa no $\mathbb{R}^{n}$, não foi provada a convergência global da seqüência $\left\{U_{k}\right\}$ para uma solução dual. Porém, foi provado que a seqüência $\left\{\mathcal{K}_{\mathrm{D}}\left(U_{k}\right)\right\}$ converge para o valor ótimo do problema dual e que os pontos limites da seqüência $\left\{U_{k}\right\}$ são soluções duais.

Além disso, é importante destacar que o teorema não garante a convergência primal da seqüência $\left\{x^{k}\right\}$ gerada pelo Algoritmo (AME). Em especial, não foi provado que os pontos limites da seqüência $\left\{x^{k}\right\}$ são viáveis para o problema primal. Entretanto, o teorema utiliza a seqüência ergódica $\left\{\bar{x}^{k}\right\}$ para garantir a convergência primal e obtém soluções de (PSDL) 
a partir dos pontos limites dessa seqüência. Uma propriedade interessante é que podemos construir de forma iterativa cada elemento da seqüência ergódica da seguinte forma:

$$
\begin{aligned}
& \bar{x}^{1}=\frac{\mu_{1}}{\nu_{1}} x^{1}=\frac{\mu_{1}}{\mu_{1}} x^{1}=x^{1}, \\
& \bar{x}^{k}=\frac{\nu_{k-1} \bar{x}^{k-1}+\mu_{k} x^{k}}{\nu_{k-1}+\mu_{k}}, \text { para } k>1 .
\end{aligned}
$$

Esse processo permite que a construção da seqüência ergódica seja feita de maneira rápida e com baixo custo computacional. Entretanto, visto que esta maneira determina a ordem em que realizamos as operações aritméticas, diminuindo nossa liberdade de escolha sobre quais termos manipulamos de cada vez, pode ser mais difícil resolver alguns problemas numéricos relacionados a erros de cancelamento e de arredondamento. 
3 UM ALGORITMO PROXIMAL INTERIOR E O MÉTODO DE MULTIPLICADORES 


\section{Capítulo 4}

\section{Métodos de penalidade e barreira para programação semidefinida}

Neste capítulo, generalizamos os conceitos estudados no capítulo anterior com o objetivo de aplicar outros métodos de multiplicadores para resolver o problema primal, com ênfase no primal linear. Para isso, é analisado um algoritmo de penalidade e barreira proposto por Mosheyev e Zibulevsky em [MZ00] para resolver esse problema. Tal algoritmo estende métodos conhecidos de lagrangianos aumentados não-quadráticos suaves como, por exemplo, o exponencial abordado no capítulo anterior e o quadrático-logarítmico introduzido por Ben-Tal e Zibulevsky em [BTZ97] para problemas de programação convexa no $\mathbb{R}^{n}$.

\subsection{Algoritmo de penalidade e barreira}

Vamos supor que as seguintes hipóteses são válidas para (PSDA):

Hipótese 4.1.1. A condição de Slater é válida, ou seja, o conjunto $\mathrm{F}_{\mathrm{P}}^{\circ}$ é não-vazio.

Hipótese 4.1.2. O conjunto de soluções do problema primal, $\mathrm{S}_{\mathrm{P}}^{*}$, é não-vazio e compacto.

Hipótese 4.1.3. A função objetivo do problema primal é duas vezes diferenciável.

Hipótese 4.1.4. O domínio efetivo da função objetivo do problema dual possui um ponto estritamente viável, isto é, $\operatorname{dom} \mathcal{K}_{\mathrm{D}} \cap \mathbb{S}_{++}^{m} \neq \emptyset$.

Sob as Hipóteses 4.1.1 e 4.1.2, nós sabemos, pelo Teorema 2.3.4, que a folga de dualidade é zero e que o conjunto de soluções do problema dual, $\mathrm{S}_{\mathrm{D}}^{*}$, é não-vazio e compacto. Vale destacar que também adotamos essas duas hipóteses no Capítulo 3 para provarmos alguns resultados sobre a convergência do método de multiplicadores exponenciais aplicado ao problema primal linear.

Associada ao método de lagrangianos aumentados apresentado mais a frente neste capítulo, adotamos uma função penalidade $\varphi: \mathbb{R} \rightarrow(-\infty,+\infty]$ com as seguintes propriedades:

$(\varphi 0) \varphi$ é uma função estritamente convexa, crescente e derivável até segunda ordem com $\operatorname{dom} \varphi=(-\infty, b)$, em que $0<b \leq \infty$; 
$(\varphi 1) \lim _{t \rightarrow b} \varphi^{\prime}(t)=\infty$

(甲2) $\lim _{t \rightarrow-\infty} \varphi^{\prime}(t)=0$

$(\varphi 3) \varphi(0)=0$

$(\varphi 4) \varphi^{\prime}(0)=1$.

Um exemplo de uma função penalidade que satisfaz as propriedades $(\varphi 0)-(\varphi 4)$ é apresentado na Figura 4.1 junto com a sua derivada.

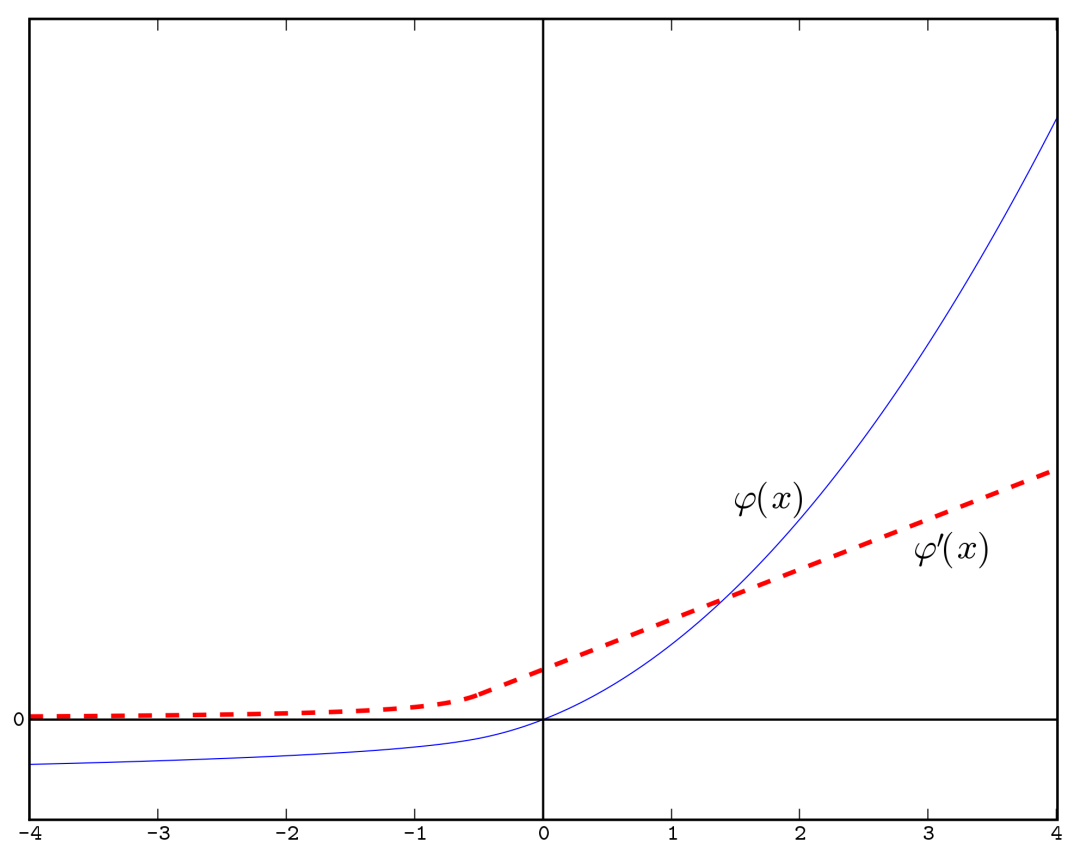

Figura 4.1: Exemplo de função penalidade.

Para cada parâmetro real $p>0$, definimos a função

$$
\varphi_{p}(t) \stackrel{\text { def }}{=} p \varphi\left(\frac{t}{p}\right)
$$

a qual também satisfaz as propriedades $(\varphi 0)-(\varphi 4)$. Além disso,

$$
p \varphi\left(\frac{t}{p}\right) \leq 0 \text { se e somente se } t \leq 0 .
$$

O algoritmo proposto em [MZ00] baseia-se no seguinte fato, apresentado no Teorema 1.3.2: a matriz $A_{0}+\mathcal{A}(x)$ é semidefinida negativa se e somente se seus autovalores são nãopositivos. Pela expressão (4.1), essa condição equivale a

$$
\varphi_{p}\left(\lambda_{i}\left(A_{0}+\mathcal{A}(x)\right)\right)=p \varphi\left(\frac{\lambda_{i}\left(A_{0}+\mathcal{A}(x)\right)}{p}\right) \leq 0, \quad i=1, \ldots, m,
$$


para todo $p>0$. Por outro lado, se $x$ não satisfaz a restrição $A_{0}+\mathcal{A}(x) \preceq 0$, então existe $j \in\{1, \ldots, m\}$ tal que $\lambda_{j}\left(A_{0}+\mathcal{A}(x)\right)>0$, o que equivale a

$$
\varphi_{p}\left(\lambda_{j}\left(A_{0}+\mathcal{A}(x)\right)\right)=p \varphi\left(\frac{\lambda_{j}\left(A_{0}+\mathcal{A}(x)\right)}{p}\right)>0,
$$

para todo $p>0$. Logo, podemos interpretar a função

$$
\sum_{i=1}^{m} \varphi_{p}\left(\lambda_{i}\left(A_{0}+\mathcal{A}(x)\right)\right)=\operatorname{tr}\left(\varphi_{p}\left(A_{0}+\mathcal{A}(x)\right)\right)
$$

como uma multa atribuída pela violação da restrição $A_{0}+\mathcal{A}(x) \preceq 0$ do problema primal, para cada $p>0$, o qual denotamos por parâmetro de penalidade. Quando reduzimos o valor do parâmetro de penalidade, a multa aplicada aos pontos inviáveis do problema primal é maior e aquela associada aos pontos viáveis diminui de forma que, para $p \rightarrow 0$, o gráfico da função penalidade $\varphi_{p}$ tende a se aproximar dos semi-eixos do ortante $\mathbb{R}_{-} \times \mathbb{R}_{+}$. Ilustramos essa situação na Figura 4.2, onde apresentamos a mesma função penalidade da Figura 4.1 com dois parâmetros de penalidade diferentes.

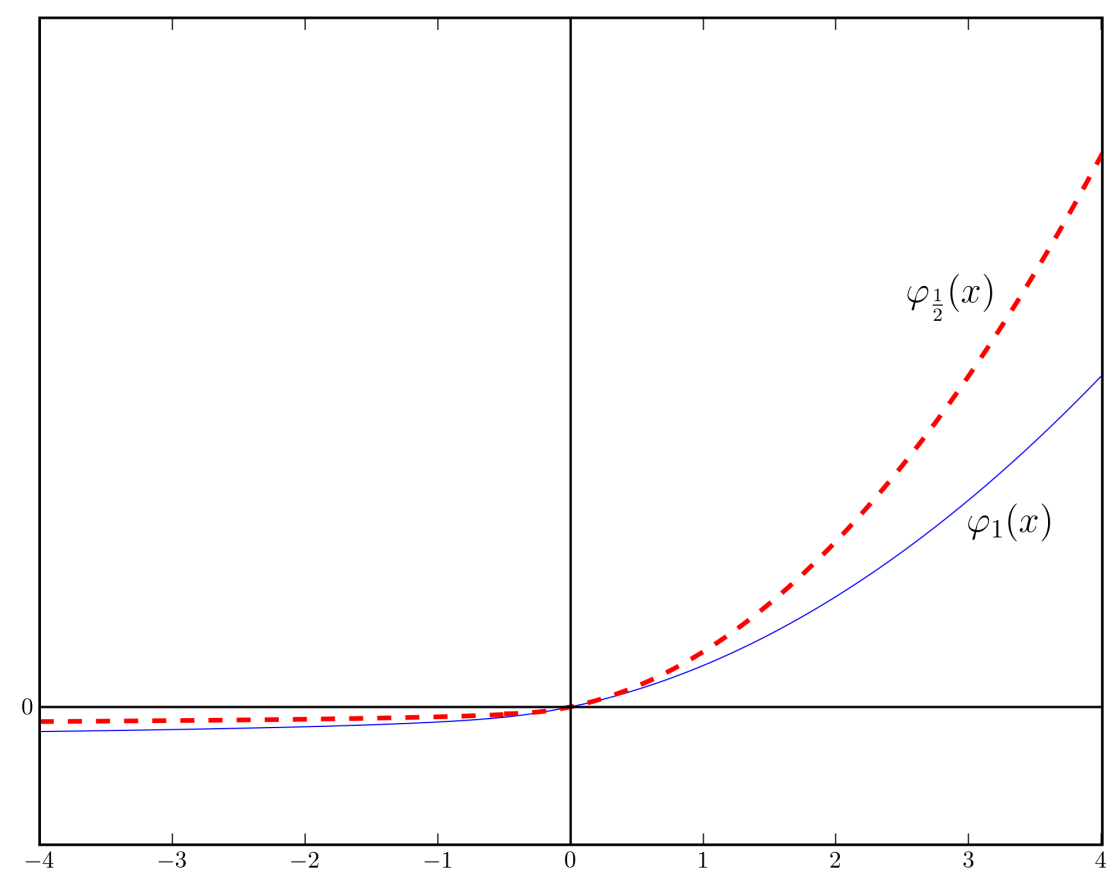

Figura 4.2: Exemplo de função penalidade.

Ao combinarmos essa multa com a função objetivo de (PSDA), definimos a função

$$
F_{p}(x) \stackrel{\text { def }}{=} f(x)+\operatorname{tr}\left(\varphi_{p}\left(A_{0}+\mathcal{A}(x)\right)\right)
$$

para cada parâmetro de penalidade $p>0$. Conseqüentemente, obtemos um problema penalizado

$$
\min _{x \in \mathbb{R}^{n}} F_{p}(x)
$$


associado a cada $p>0$. Sob hipóteses de regularidade razoáveis, quando $p \rightarrow 0$, é possível demonstrar que o caminho de minimizadores do problema (4.4) converge para o conjunto de soluções do problema primal. Entretanto, para valores de $p$ próximos de zero, pode haver instabilidade numérica na resolução do problema penalizado, o que dificulta a obtenção de uma solução.

Por outro lado, podemos evitar que o parâmetro de penalidade se aproxime demasiadamente de zero se utilizarmos informações do problema dual. Em particular, além de obtermos um ponto $x^{*} \in \mathbb{R}^{n}$ que seja uma solução do problema primal, também procuramos obter uma matriz $U^{*} \in \mathbb{S}^{m}$ que seja uma solução do problema dual. Assim, estamos interessados em obter um par $\left(x^{*}, U^{*}\right) \in \mathbb{R}^{n} \times \mathbb{S}^{m}$ que satisfaça as condições de otimalidade do Teorema 2.3.2. Veremos agora um método de lagrangianos aumentados suaves que possui tais propriedades.

Pelo Teorema 1.3.5, sabemos que, para toda matriz $U \in \mathbb{S}_{+}^{m}$, existe uma única matriz $U^{1 / 2} \in \mathbb{S}_{+}^{m}$ tal que $U=\left(U^{1 / 2}\right)^{2}$. Logo, para toda matriz semidefinida positiva $U$, podemos usar essa igualdade para reescrever a função lagrangiana $L$ associada ao problema primal como

$$
\begin{aligned}
\mathrm{L}(x, U) & =\mathrm{L}\left(x,\left(U^{1 / 2}\right)^{2}\right) \\
& =f(x)+\left\langle\left(U^{1 / 2}\right)^{2}, A_{0}+\mathcal{A}(x)\right\rangle \\
& =f(x)+\operatorname{tr}\left(U^{1 / 2} U^{1 / 2}\left(A_{0}+\mathcal{A}(x)\right)\right) \\
& =f(x)+\operatorname{tr}\left(U^{1 / 2}\left(A_{0}+\mathcal{A}(x)\right) U^{1 / 2}\right) \quad \text { [equação (1.1)]. }
\end{aligned}
$$

Sabemos que a matriz $U^{1 / 2}\left(A_{0}+\mathcal{A}(x)\right) U^{1 / 2}$ é simétrica pelo Teorema 1.2.1. Além disso, novamente pelo Teorema 1.3.5, se $U \succ 0$, então $U^{1 / 2} \succ 0$ também. Como resultado, se $U \succ 0$, então a matriz $U^{1 / 2}\left(A_{0}+\mathcal{A}(x)\right) U^{1 / 2}$ será semidefinida negativa se e somente se $x$ for um ponto viável do problema primal, ou seja, se $A_{0}+\mathcal{A}(x)$ for semidefinida negativa também. Logo, de maneira similar à feita na equação (4.2), podemos atribuir uma penalidade quando a matriz $U^{1 / 2}\left(A_{0}+\mathcal{A}(x)\right) U^{1 / 2}$ não for semidefinida negativa:

$$
\sum_{i=1}^{m} \varphi_{p}\left(\lambda_{i}\left(U^{1 / 2}\left(A_{0}+\mathcal{A}(x)\right) U^{1 / 2}\right)\right)=\operatorname{tr}\left(\varphi_{p}\left(U^{1 / 2}\left(A_{0}+\mathcal{A}(x)\right) U^{1 / 2}\right)\right) .
$$

Observemos que o fato da matriz $U^{1 / 2}\left(A_{0}+\mathcal{A}(x)\right) U^{1 / 2}$ ser simétrica implica, pelo Teorema 1.2.1, que todos os seus autovalores são reais. Logo, $\varphi_{p}\left(U^{1 / 2}\left(A_{0}+\mathcal{A}(x)\right) U^{1 / 2}\right)$ está bem definida, visto que o domínio de $\varphi$ é $\mathbb{R}$.

Assim, ao combinarmos a multa definida pela expressão (4.5) com a função objetivo de (PSDA) e seguindo as idéias discutidas em [BTZ97] e [MZ00], nós relacionamos o problema primal a um lagrangiano aumentado $\mathrm{L}_{p}^{+}: \mathbb{R}^{n} \times \mathbb{S}^{m} \rightarrow(-\infty, \infty]$ definido por

$$
\mathrm{L}_{p}^{+}(x, V) \stackrel{\text { def }}{=} f(x)+\operatorname{tr}\left(\varphi_{p}\left(V\left(A_{0}+\mathcal{A}(x)\right) V\right)\right),
$$

para todo parâmetro de penalidade $p>0$. Pela Hipótese 4.1.3 e pelo Corolário 1.5.8, tal função é duas vezes diferenciável. 
Podemos agora introduzir o algoritmo apresentado por Mosheyev e Zibulevsky em [MZ00] para resolver (PSDA). Esse algoritmo é iterativo e baseado em métodos de penalidade e barreira abordados em um artigo de Ben-Tal e Zibulevsky [BTZ97]. Por tal motivo, ele é denotado por Algoritmo de Penalidade e Barreira (APB) para o problema primal.

\section{Algoritmo 4.1 (APB).}

(i) Escolha uma matriz $U_{0} \in \mathbb{S}_{++}^{m}$, um parâmetro real $p_{0}>0$ e uma seqüência $\left\{\pi^{k}\right\}$ de atualização dos parâmetros de penalidades tal que $0<\pi^{k} \leq 1$, para todo $k \geq 0$. Além disso, seja $V_{0} \stackrel{\text { def }}{=} U_{0}^{1 / 2}$.

(ii) A cada iteração $k \geq 0$, minimize o lagrangiano aumentado definido em (4.6) com relação a $x$ :

$$
x^{k+1} \in \underset{x \in \mathbb{R}^{n}}{\operatorname{argmin}} \mathrm{L}_{p_{k}}^{+}\left(x, V_{k}\right) .
$$

Então, atualize a matriz de multiplicadores $U_{k}$ e o parâmetro de penalidade $p_{k}$ a partir das fórmulas

$$
\begin{aligned}
V_{k+1} & =\left(V_{k} \varphi_{p_{k}}^{\prime}\left(V_{k}\left(A_{0}+\mathcal{A}\left(x^{k+1}\right)\right) V_{k}\right) V_{k}\right)^{1 / 2}, \\
U_{k+1} & =V_{k+1}^{2} \\
p_{k+1} & =\pi^{k} p_{k} .
\end{aligned}
$$

A motivação para a escolha da fórmula de atualização da matriz de multiplicadores $V_{k}$ gerada pelo algoritmo vem da condição de otimalidade (2.8a) do Teorema 2.3.2:

$$
0 \in \partial_{x} \mathrm{~L}\left(x^{k+1}, U_{k+1}\right) .
$$

Já que, pela Hipótese 4.1.3, $f$ é duas vezes diferenciável, a expressão acima é equivalente a

$$
\nabla_{x} \mathrm{~L}\left(x^{k+1}, U_{k+1}\right)=\nabla f\left(x^{k+1}\right)+\mathcal{A}^{*}\left(U_{k+1}\right)=0 .
$$

Por outro lado, a partir da Proposição 1.4.6, uma condição necessária e suficiente para que $x^{k+1}$ minimize o lagrangiano aumentado $L_{p_{k}}^{+}$em relação a $x$ na expressão (4.7) é que

$$
0=\nabla_{x} \mathrm{~L}_{p_{k}}^{+}\left(x^{k+1}, V_{k}\right)=\nabla f\left(x^{k+1}\right)+\mathcal{A}^{*}\left(V_{k} \varphi_{p_{k}}^{\prime}\left(V_{k}\left(A_{0}+\mathcal{A}\left(x^{k+1}\right)\right) V_{k}\right) V_{k}\right) .
$$

Dessa forma, ao igualar as expressões (4.11) e (4.12) e pelo fato de que $U_{k}=V_{k}^{2}$ para todo inteiro $k \geq 0$, obtemos

$$
\mathcal{A}^{*}\left(V_{k} \varphi_{p_{k}}^{\prime}\left(V_{k}\left(A_{0}+\mathcal{A}\left(x^{k+1}\right)\right) V_{k}\right) V_{k}\right)=\mathcal{A}^{*}\left(U_{k+1}\right)=\mathcal{A}^{*}\left(V_{k+1}^{2}\right)
$$

A fórmula de atualização da matriz de multiplicadores definida pela equação (4.8) surge como uma escolha natural a partir da equação acima. Provamos posteriormente que essa escolha é a única possível. 


\subsubsection{Escolha da função penalidade/barreira}

A partir da apresentação feita no início desta seção, podemos ver que a escolha da função penalidade $\varphi$ define qual lagrangiano aumentado estamos usando. Como conseqüência, tal escolha tem muita influência sobre o desempenho do algoritmo de penalidade e barreira adotado. Mostramos agora algumas das funções penalidades mais usadas e conhecidas em programação não-linear, comentando se podemos utilizá-las no algoritmo apresentado há pouco. Todas as funções que mencionamos são baseadas naquelas discutidas em [MZ00]. Entretanto, devido à mudança de sinal na restrição matricial $A_{0}+\mathcal{A}(x)$ do problema primal, fizemos adaptações nas funções penalidades em relação ao artigo de Mosheyev e Zibulevsky com base no artigo de Ben-Tal e Zibulevsky[BTZ97].

\section{Função barreira logarítmica:}

$$
\varphi(t)=\log t
$$

Para esta função, temos a penalidade

$$
\begin{aligned}
\operatorname{tr}\left(\varphi\left(A_{0}+\mathcal{A}(x)\right)\right) & =\sum_{i=1}^{m} \log \lambda_{i}\left(A_{0}+\mathcal{A}(x)\right) \\
& =\log \prod_{i=1}^{m} \lambda_{i}\left(A_{0}+\mathcal{A}(x)\right) \\
& =\log \operatorname{det}\left(A_{0}+\mathcal{A}(x)\right) \quad \text { [Proposição 1.1.3]. }
\end{aligned}
$$

A partir do Proposição 1.5.5, obtemos o gradiente desta função:

$$
\nabla \operatorname{tr}(\varphi(A))=\varphi^{\prime}(A)=A^{-1} .
$$

Apesar de muito conhecida e utilizada em métodos de pontos interiores, essa função não pode ser utilizada no Algoritmo (APB), visto que não satisfaz as propriedades $\varphi 2$, $\varphi 3$ e $\varphi 4$.

\section{Função barreira modificada:}

$$
\varphi(t)=-\log (1-t), \quad-\infty<t<1 .
$$

Para esta função, temos a penalidade

$$
\begin{aligned}
\operatorname{tr}\left(\varphi\left(A_{0}+\mathcal{A}(x)\right)\right) & =-\sum_{i=1}^{m} \log \left(1-\lambda_{i}\left(A_{0}+\mathcal{A}(x)\right)\right) \\
& =-\log \prod_{i=1}^{m}\left(1-\lambda_{i}\left(A_{0}+\mathcal{A}(x)\right)\right) \\
& =-\log \operatorname{det}\left(I-\left(A_{0}+\mathcal{A}(x)\right)\right)
\end{aligned}
$$

cujo gradiente é

$$
\nabla \operatorname{tr}(\varphi(A))=\varphi^{\prime}(A)=(I-A)^{-1} \quad[\text { Proposição 1.5.5]. }
$$

Essa função satisfaz as propriedades $\varphi 0-\varphi 4$ e, assim, pode ser usada no Algoritmo (APB). 


\section{Função penalidade exponencial:}

$$
\varphi(t)=e^{t}-1
$$

Para esta função, temos a penalidade

$$
\begin{aligned}
\operatorname{tr}\left(\varphi\left(A_{0}+\mathcal{A}(x)\right)\right) & =\sum_{i=1}^{m}\left(e^{\lambda_{i}\left(A_{0}+\mathcal{A}(x)\right)}-1\right) \\
& =\operatorname{tr}\left(e^{\left(A_{0}+\mathcal{A}(x)\right)}-I\right),
\end{aligned}
$$

cujo gradiente é dado por

$$
\nabla \operatorname{tr}(\varphi(A))=\varphi^{\prime}(A)=e^{A} \quad[\text { Proposição 1.5.5]. }
$$

Tal função satisfaz $\varphi 0-\varphi 4$ e, então, pode ser utilizada no Algoritmo (APB). O lagrangiano aumentado associado a essa função é semelhante ao estudado no Capítulo 3, onde foi utilizado para resolver os problemas primal e dual linear, especificamente.

4. Função penalidade quadrática-logarítmica mista: Para esta função, temos a penalidade

$$
\varphi(t)= \begin{cases}\frac{a}{2} t^{2}+b t+c, & \text { se } t \geq \tau, \\ d \log (e-t)+f, & \text { se } t<\tau,\end{cases}
$$

em que $\tau \in(-1,1)$ é um parâmetro que determina o ponto de junção. Os coeficientes $a, b, c, d, e, f$ são determinados pelas propriedades de que $\varphi$ seja duas vezes diferenciável em $t=\tau, \varphi(0)=0, \varphi^{\prime}(0)=1$ e $\varphi^{\prime \prime}(0)=1$. Para $\tau=-1 / 2$, temos

$$
\varphi(t)= \begin{cases}\frac{t^{2}}{2}+t, & \text { se } t \geq-\frac{1}{2}, \\ -\frac{1}{4} \log (-2 t)-\frac{3}{8}, & \text { caso contrário. }\end{cases}
$$

Tal função para $\tau=-1 / 2$ e a sua derivada são ilustradas na Figura 4.1.

A função penalidade quadrática-logarítmica também satisfaz as propriedades $\varphi 0-\varphi 4$ e, assim, pode ser usada no algoritmo proposto. Essa escolha da função penalidade tem como vantagem o fato de que a segunda derivada de $\varphi$ é contínua e limitada para todo $t$ real. Tal propriedade é importante para métodos que minimização que utilizam a segunda derivada de uma função como, por exemplo, o método de Newton.

\subsection{Análise dual do algoritmo de penalidade e barreira}

Abordamos agora uma análise dual do algoritmo de penalidade e barreira apresentado. Tal análise é baseada na relação entre (APB) e um algoritmo de ponto proximal adequado aplicado sobre o problema dual. Os resultados que discutimos seguem as idéias desenvolvidas em [MZ00, BTZ97].

Para todo parâmetro de penalidade $p>0$, denotamos por $\psi_{p}$ a função convexo conjugada de uma função penalidade $\varphi_{p}$ que satisfaça as propriedades $(\varphi 0)-(\varphi 4)$ :

$$
\psi_{p}(s) \stackrel{\text { def }}{=} \varphi_{p}^{*}(s)=\sup _{t \in \mathbb{R}}\left\{s t-\varphi_{p}(t)\right\} .
$$


Para mantermos coerência com a notação utilizada para a função penalidade $\varphi_{p}$, seja $\psi \stackrel{\text { def }}{=}$ $\psi_{1}$. A próxima proposição relaciona $\psi_{p}$ e $\psi$ para todo $p>0$ :

Proposição 4.2.1. Sejam $p>0$ e $\varphi_{p}$ uma função penalidade que satisfaça as propriedades $(\varphi 0)-(\varphi 4)$. Então,

$$
\psi_{p}(s)=p \psi(s) .
$$

Demonstração. A partir das definições de $\varphi_{p}$ e de $\psi_{p}$, temos

$$
\begin{aligned}
\psi_{p}(s) & =\sup _{t \in \mathbb{R}}\left\{s t-\varphi_{p}(t)\right\}=\sup _{t \in \mathbb{R}}\left\{s t-p \varphi\left(\frac{t}{p}\right)\right\}=\sup _{t \in \mathbb{R}}\left\{p\left(s \frac{t}{p}-\varphi\left(\frac{t}{p}\right)\right)\right\} \\
& =p \sup _{t \in \mathbb{R}}\left\{s \frac{t}{p}-\varphi\left(\frac{t}{p}\right)\right\}=p \sup _{t \in \mathbb{R}}\{s t-\varphi(t)\}=p \varphi^{*}(s)=p \psi(s),
\end{aligned}
$$

resultando que $\psi_{p}(s)=p \psi(s)$.

Um outro fato bastante conhecido e útil sobre $\psi_{p}$ que usamos a seguir é que $\psi_{p}^{\prime}=\left(\varphi_{p}^{\prime}\right)^{-1}$ (veja, por exemplo, [Roc70, Corolário 23.5.1]). Ademais, a partir das propriedades $(\varphi 0)-$ $(\varphi 4)$, obtemos as seguintes propriedades sobre $\psi_{p}$, para todo $p>0$ :

$(\psi 0) \psi_{p}$ é uma função estritamente convexa e duas vezes diferenciável em $(0, \infty)$;

$(\psi 1) \psi_{p}(1)=0$

$(\psi 2) \quad \psi_{p}^{\prime}(1)=0$

$(\psi 3) \lim _{s \rightarrow 0^{+}} \psi_{p}^{\prime}(s)=-\infty, \quad \psi_{p}(s)=+\infty$, para todo $s \leq 0, \quad \lim _{s \rightarrow \infty} \psi_{p}(s)=+\infty ;$

$(\psi 4) \min _{s \in \mathbb{R}}\left\{\psi_{p}(s)\right\}=\min _{s>0}\left\{\psi_{p}(s)\right\}=\psi_{p}(1)=0$.

Chamamos $\psi_{p}$ de função barreira devido à propriedade $(\psi 3)$.

Como exemplo, tomemos a conjugada da função penalidade quadrática-logarítmica definida na seção anterior, para $\tau=-1 / 2$ :

$$
\varphi^{*}(s)= \begin{cases}\frac{1}{2}(s-1)^{2}, & \text { se } s \geq \frac{1}{2} \\ \frac{1}{8}-\frac{1}{4} \log (2 s), & \text { se } 0<s<\frac{1}{2} \\ +\infty, & \text { caso contrário. }\end{cases}
$$

Ilustramos essa função na Figura 4.3 junto com a sua derivada.

Abordamos agora uma relação entre a restrição matricial do problema primal e a função objetivo do problema dual. Particularmente, o próximo resultado associa a matriz que determina uma das restrições do problema primal no ponto $x^{k+1}$, definido pela expressão (4.7), com o subdiferencial da função objetivo do problema dual em $U_{k+1}$, definida pela expressão (4.9).

Proposição 4.2.2 ([MZ00, Proposição 1]). A matriz $A_{0}+\mathcal{A}\left(x^{k+1}\right)$ pertence ao subdiferencial da função objetivo dual no ponto $U_{k+1}$ :

$$
A_{0}+\mathcal{A}\left(x^{k+1}\right) \in \partial \mathcal{K}_{\mathrm{D}}\left(U_{k+1}\right) .
$$




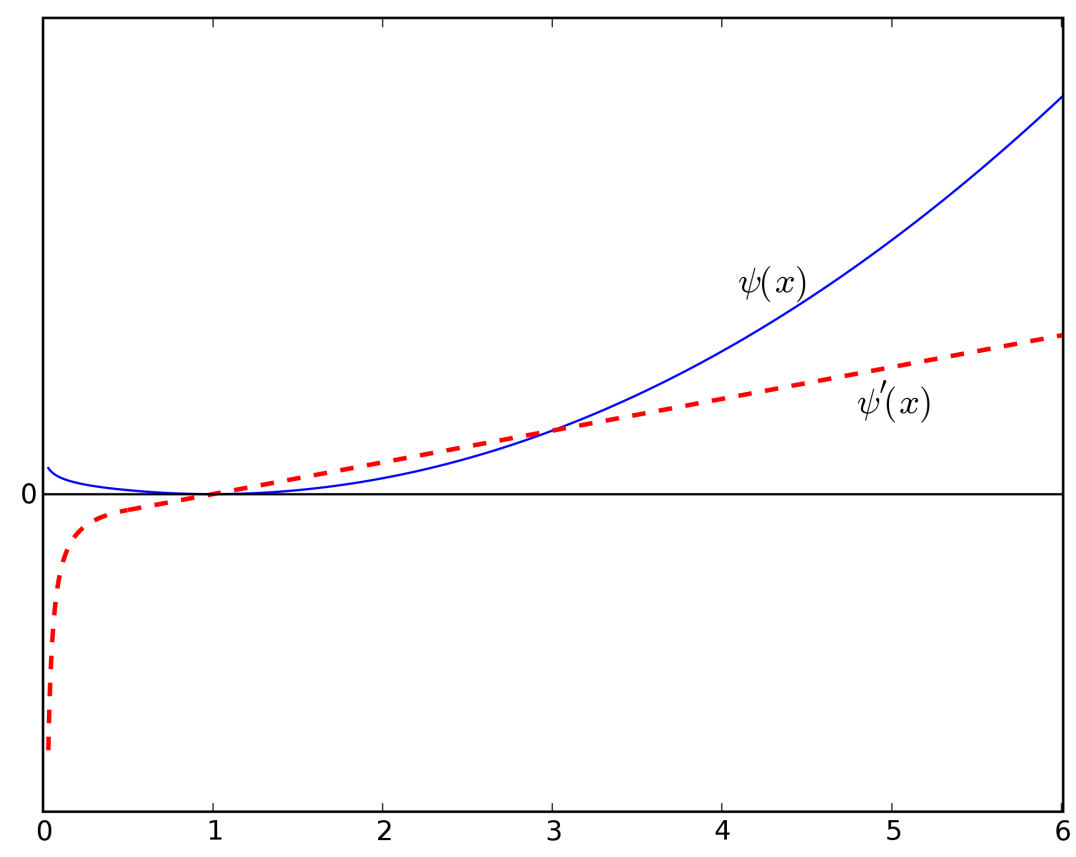

Figura 4.3: Exemplo de função barreira.

Demonstração. A prova que fazemos a seguir é semelhante a uma parte da demonstração do Lema 3.2.2. A partir da definição da função lagrangiana, para toda matriz $U \in \mathbb{S}^{m}$, nós sabemos que

$$
\begin{aligned}
\mathcal{K}_{\mathrm{D}}(U) & =\inf _{x \in \mathbb{R}^{n}} \mathrm{~L}(x, U) \\
& \leq \mathrm{L}\left(x^{k+1}, U\right)=f\left(x^{k+1}\right)+\left\langle U, A_{0}+\mathcal{A}\left(x^{k+1}\right)\right\rangle \\
& =f\left(x^{k+1}\right)+\left\langle U_{k+1}, A_{0}+\mathcal{A}\left(x^{k+1}\right)\right\rangle+\left\langle U-U_{k+1}, A_{0}+\mathcal{A}\left(x^{k+1}\right)\right\rangle \\
& =\mathrm{L}\left(x^{k+1}, U_{k+1}\right)+\left\langle U-U_{k+1}, A_{0}+\mathcal{A}\left(x^{k+1}\right)\right\rangle,
\end{aligned}
$$

resultando que $\mathcal{K}_{\mathrm{D}}(U) \leq \mathrm{L}\left(x^{k+1}, U_{k+1}\right)+\left\langle U-U_{k+1}, A_{0}+\mathcal{A}\left(x^{k+1}\right)\right\rangle$. Por outro lado, a partir da condição de otimalidade expressa pela equação (4.11), temos $\mathcal{K}_{\mathrm{D}}\left(U_{k+1}\right)=$ $\mathrm{L}\left(x^{k+1}, U_{k+1}\right)$. Assim,

$$
\mathcal{K}_{\mathrm{D}}(U) \leq \mathcal{K}_{\mathrm{D}}\left(U_{k+1}\right)+\left\langle U-U_{k+1}, A_{0}+\mathcal{A}\left(x^{k+1}\right)\right\rangle
$$

implicando que $A_{0}+\mathcal{A}\left(x^{k+1}\right) \in \partial \mathcal{K}_{\mathrm{D}}\left(U_{k+1}\right)$. 


\subsubsection{Conexão do método de lagrangiano aumentado com um algoritmo de ponto proximal}

Consideremos uma iteração do seguinte método de ponto proximal para resolver o problema dual:

$$
\tilde{U}_{k+1} \in \underset{U \succeq 0}{\operatorname{argmax}}\left\{\mathcal{K}_{\mathrm{D}}(U)-D_{p_{k}}\left(U, \tilde{U}_{k}\right)\right\}
$$

em que $\tilde{U}_{0}=U_{0} \succ 0$ e $D_{p_{k}}: \mathbb{S}_{+}^{m} \times \mathbb{S}_{++}^{m} \rightarrow \mathbb{R}$ é uma regularização definida por

$$
D_{p}(U, W) \stackrel{\text { def }}{=} \operatorname{tr}\left(\varphi_{p}^{*}\left(W^{-1 / 2} U W^{-1 / 2}\right)\right)=\operatorname{tr}\left(\psi_{p}\left(W^{-1 / 2} U W^{-1 / 2}\right)\right),
$$

para todo real $p>0$. Para simplificar a notação, denotamos por $D$ a função $D_{1}$. Logo, pela Proposição 4.2.1, segue que $D_{p}=p D$, para todo $p>0$.

A partir das propriedades $(\psi 0)-(\psi 4)$, as seguintes afirmações são válidas para toda matriz $W \succ 0$ :

(D0) a função $D(\cdot, W)$ é estritamente convexa e duas vezes diferenciável;

(D1) para toda matriz $U \in \mathbb{S}_{+}^{m}$,

(i) $D(U, W) \geq 0$;

(ii) $D(U, W)=0$ se e somente se $U=W$;

(D2) para toda matriz $U \in \mathbb{S}^{m}$, temos:

(i) se $U \nsucc 0$, então $D(U, W)=+\infty$;

(ii) $\lim _{\|W\| \rightarrow \infty} D(U, W)=+\infty$.

Além disso, para toda matriz $\bar{U} \succ 0$,

(D3) a função $D(\bar{U}, \cdot)$ tem conjuntos de nível limitados;

(D4) a função $D(\bar{U}, \cdot)$ é contínua em $\bar{U}$;

(D5) para qualquer seqüência $\left\{W_{k}\right\} \subseteq \mathbb{S}_{++}^{m}$, temos $D\left(\bar{U}, W_{k}\right) \rightarrow 0$ se e somente se $W_{k} \rightarrow \bar{U}$.

A prova desses resultados segue as idéias abordadas no Capítulo 3.

Para fazermos a conexão entre o Algoritmo (APB) e o algoritmo de ponto proximal definido pela iteração (4.14), abordamos agora um resultado análogo ao Teorema 3.1.4 aplicado a esse algoritmo de ponto proximal. Por $(\psi 0)$ e $(\psi 3)$, a função objetivo do método de ponto proximal na $k$-ésima iteração, $\mathcal{K}_{\mathrm{D}}(\cdot)-D_{p_{k}}\left(\cdot, \tilde{U}_{k}\right)$, é estritamente côncava e coerciva. Logo, possui um único maximizador. Pela Proposição 1.4.6, uma condição necessária e suficiente para que $U_{k+1}$ seja a solução do método de ponto proximal na iteração $k+1$ é

$$
0 \in \partial\left(\mathcal{K}_{\mathrm{D}}\left(\tilde{U}_{k+1}\right)-D_{p_{k}}\left(\tilde{U}_{k+1}, \tilde{U}_{k}\right)+\delta_{\mathbb{S}_{+}^{m}}\left(U_{k+1}\right)\right) .
$$

Sob as Hipóteses 4.1.1 e 4.1.2, sabemos que o valor ótimo do problema dual é finito e igual ao do problema primal. Além disso, pela Hipótese 4.1.4, sabemos que existe $U^{\circ} \succ 0$ tal 
que $\mathcal{K}_{\mathrm{D}}\left(U^{\circ}\right)$ é finita. Logo, a partir da regra da soma de subdiferenciais ([Roc70, Teorema 23.8]), a expressão (4.15) é equivalente a

$$
0 \in \partial \mathcal{K}_{\mathrm{D}}\left(\tilde{U}_{k+1}\right)-\partial_{1} D_{p_{k}}\left(\tilde{U}_{k+1}, \tilde{U}_{k}\right)+\partial \delta_{\mathbb{S}_{+}^{m}}\left(U_{k+1}\right)
$$

De maneira análoga à discutida no capítulo anterior, obtemos $\partial_{1} D_{p_{k}}\left(U, \tilde{U}_{k}\right)=\emptyset$ para toda matriz $U \in \mathbb{S}_{+}^{m} \backslash \mathbb{S}_{++}^{m}$, implicando que $\tilde{U}_{k+1} \succ 0$. Logo, a partir das Proposições $1.4 .9 \mathrm{e}$ 1.3.8, temos $\partial \delta_{\mathbb{S}_{+}^{m}}\left(U_{k+1}\right)=\{0\}$. Ademais, já que a regularização é diferenciável em relação ao primeiro argumento, sabemos que

$$
\begin{aligned}
\nabla_{1} D_{p_{k}}\left(\tilde{U}_{k+1}, \tilde{U}_{k}\right) & =\tilde{U}_{k}^{-1 / 2}\left(\psi_{p_{k}}^{\prime}\left(\tilde{U}_{k}^{-1 / 2} \tilde{U}_{k+1} \tilde{U}_{k}^{-1 / 2}\right)\right) \tilde{U}_{k}^{-1 / 2} \\
& =\tilde{U}_{k}^{-1 / 2}\left(\left(\varphi_{p_{k}}^{\prime}\right)^{-1}\left(\tilde{U}_{k}^{-1 / 2} \tilde{U}_{k+1} \tilde{U}_{k}^{-1 / 2}\right)\right) \tilde{U}_{k}^{-1 / 2}
\end{aligned}
$$

Assim, a expressão (4.16) pode ser escrita como

$$
0 \in \partial \mathcal{K}_{\mathrm{D}}\left(\tilde{U}_{k+1}\right)+\left\{-\tilde{U}_{k}^{-1 / 2}\left(\left(\varphi_{p_{k}}^{\prime}\right)^{-1}\left(-\tilde{U}_{k}^{-1 / 2} U_{k+1} \tilde{U}_{k}^{-1 / 2}\right)\right) \tilde{U}_{k}^{-1 / 2}\right\} .
$$

Mostremos agora que a seqüência produzida por esse método de ponto proximal é a mesma gerada pelo Algoritmo de penalidade e barreira para (PSDA). A partir da equação (4.8) e do fato de que $U_{k}=V_{k}^{2}$ para todo inteiro $k \geq 0$, temos

$$
A_{0}+\mathcal{A}\left(x^{k+1}\right)=U_{k}^{-1 / 2}\left(\left(\varphi_{p_{k}}^{\prime}\right)^{-1}\left(U_{k}^{-1 / 2} U_{k+1} U_{k}^{-1 / 2}\right)\right) U_{k}^{-1 / 2} .
$$

Quando combinamos essa equação com a Proposição 4.2.2, obtemos

$$
0 \in \partial \mathcal{K}_{\mathrm{D}}\left(U_{k+1}\right)+\left\{-U_{k}^{-1 / 2}\left(\left(\varphi_{p_{k}}^{\prime}\right)^{-1}\left(U_{k}^{-1 / 2} U_{k+1} U_{k}^{-1 / 2}\right)\right) U_{k}^{-1 / 2}\right\} .
$$

a qual é semelhante à expressão (4.17) com $\tilde{U}_{k}$ substituída por $U_{k}$. Então, já que $\tilde{U}_{0}=U_{0}$, a seqüência $\left\{U_{k}\right\}$ gerada por (APB) é igual a $\left\{\tilde{U}_{k}\right\}$, definida pelo método de ponto proximal apresentado na expressão (4.14).

Agora, podemos usar esse resultado para apresentar uma proposição que associa o valor ótimo do problema primal com a seqüência de valores da função objetivo dual quando avaliada nas matrizes $U_{k}$ geradas pelo algoritmo de penalidade e barreira.

Proposição 4.2.3 ([MZ00, Proposição 2]). Consideremos a seqüência $\left\{U_{k}\right\}$ de multiplicadores gerada pelo método de penalidade e barreira descrito por (4.7)-(4.10). Então, a seqüência $\left\{\mathcal{K}_{\mathrm{D}}\left(U_{k}\right)\right\}$ é não-decrescente e limitada superiormente pelo valor ótimo do problema primal.

Demonstração. A partir da conexão que fizemos entre o Algoritmo de penalidade e barreira para (PSDA) e o método de ponto proximal para resolver o problema dual, obtemos

$$
\begin{aligned}
\mathcal{K}_{\mathrm{D}}\left(U_{k+1}\right)-D_{k}\left(U_{k+1}, U_{k}\right) & \geq \mathcal{K}_{\mathrm{D}}\left(U_{k}\right)-D_{k}\left(U_{k}, U_{k}\right) & \\
& =\mathcal{K}_{\mathrm{D}}\left(U_{k}\right), & \text { [propriedade } D 1(i i)]
\end{aligned}
$$


para todo inteiro $k \geq 0$. Logo,

$$
\mathcal{K}_{\mathrm{D}}\left(U_{k+1}\right)-\mathcal{K}_{\mathrm{D}}\left(U_{k}\right) \geq D_{k}\left(U_{k+1}, U_{k}\right) \geq 0, \quad \text { [propriedade } D 1(i) \text { ] }
$$

resultando que a seqüência $\left\{\mathcal{K}_{\mathrm{D}}\left(U_{k}\right)\right\}$ é monótona não-decrescente. Assim, a partir desse resultado e devido ao Teorema 2.2.1 e à hipótese de $\mathrm{S}_{\mathrm{P}}^{*}$ ser não-vazio, podemos ver que $\lim _{k \rightarrow \infty} \mathcal{K}_{\mathrm{D}}\left(U_{k}\right)$ existe e é menor ou igual ao valor ótimo do problema primal, $\mathrm{f}_{*}^{\mathrm{P}}$.

É importante ressaltar que no artigo de Mosheyev e Zibulevsky [MZ00], não há resultados que garantam a convergência da seqüência $\left\{U_{k}\right\}$ para uma solução do problema dual.

\subsubsection{Considerações sobre o algoritmo de penalidade e barreira}

Conforme já vimos no início deste capítulo, o algoritmo proposto por Mosheyev e Zibulevsky emprega um lagrangiano aumentado com o termo de penalidade

$$
\operatorname{tr} \varphi_{p}\left(U^{1 / 2}\left(A_{0}+\mathcal{A}(x)\right) U^{1 / 2}\right),
$$

cuja regularização aplicada no problema dual é dada por

$$
D_{p}(U, W)=\operatorname{tr} \varphi_{p}^{*}\left(W^{-1 / 2} U W^{-1 / 2}\right) .
$$

Com o intuito de entendermos melhor o desempenho do método, decidimos comparar essa penalidade com a apresentada em [BTZ97].

Para simplificar a análise, consideramos o caso em que $m=1$, ou seja, em que as matrizes são escalares. Assim, o termo com papel de penalidade do lagrangiano aumentado torna-se

$$
\varphi_{p}\left(u^{1 / 2}\left(a_{0}+a^{T} x\right) u^{1 / 2}\right)=\varphi_{p}\left(u\left(a_{0}+a^{T} x\right)\right),
$$

enquanto a regularização da equação (4.18) é

$$
D_{p}(u, w)=\varphi_{p}^{*}\left(w^{-1 / 2} u w^{-1 / 2}\right)=\varphi_{p}^{*}\left(\frac{u}{w}\right),
$$

para quaisquer $u \geq 0$ e $w>0$. Por outro lado, o lagrangiano aumentado abordado em [BTZ97] possui o termo com papel de penalidade

$$
u \varphi_{p}\left(a_{0}+a^{T} x\right)
$$

cuja regularização associada é dada por

$$
\widetilde{D}_{p}(u, w)=w \varphi_{p}^{*}\left(\frac{u}{w}\right) .
$$

Ao compararmos os termos que atuam como penalidade nas expressões (4.19) e (4.20), constatamos que, no primeiro, a função penalidade é aplicada sobre o produto $u\left(a_{0}+a^{T} x\right)$, enquanto no segundo ela é aplicada sobre a restrição $a_{0}+a^{T} x$ e multiplicada por $u$. Então, o multiplicador $u$ atua como um parâmetro de penalidade na expressão (4.19), o que não 
acontece nos métodos usuais de lagrangianos aumentados, em que o multiplicador não atua diretamente na restrição, mas de forma linear na penalidade aplicada sobre ela como, por exemplo, na expressão (4.20). A partir da definição de função penalidade feita pelas propriedades $(\varphi 0)-(\varphi 4)$ e para valores elevados de $u$, podemos interpretar que o termo da expressão (4.19) impõe uma penalidade elevada para pontos inviáveis do problema primal, de forma que, para fins práticos, o algoritmo aproxima-se de métodos de penalidade "pura" para resolver o problema primal.

Ao estudarmos as duas regularizações associadas, vemos que $\widetilde{D}_{p}(u, w)=w D_{p}(u, w)$, implicando que, para $w>1$ e conforme elevamos o valor desse argumento, a regularização utilizada por Mosheyev e Zibulevsky age de forma cada vez mais suave comparada à empregada por Ben-Tal e Zibulevsky no método de ponto proximal aplicado ao dual. Ilustramos o efeito do aumento de $w$ sobre as regularizações na Figura 4.4, onde fixamos $w=4$ e apresentamos $\widetilde{D}_{p}$ e $D_{p}$, associadas à função penalidade quadrático-logarítmica definida na subseção 4.1 .1 para $\tau=\frac{1}{2}$ e $p=1$ :

$$
\begin{aligned}
& D(u, w)= \begin{cases}\frac{(u-w)^{2}}{2 w^{2}}, & \text { se } \frac{u}{w} \geq \frac{1}{2}, \\
\frac{1}{8}-\frac{1}{4} \log (2 u)+\frac{1}{4} \log w, & \text { se } 0<\frac{u}{w}<\frac{1}{2}, \\
+\infty, & \text { caso contrário. }\end{cases} \\
& \widetilde{D}(u, w)= \begin{cases}\frac{(u-w)^{2}}{2 w}, & \text { se } \frac{u}{w} \geq \frac{1}{2}, \\
w\left(\frac{1}{8}-\frac{1}{4} \log (2 u)+\frac{1}{4} \log w\right), & \text { se } 0<\frac{u}{w}<\frac{1}{2}, \\
+\infty, & \text { caso contrário. }\end{cases}
\end{aligned}
$$

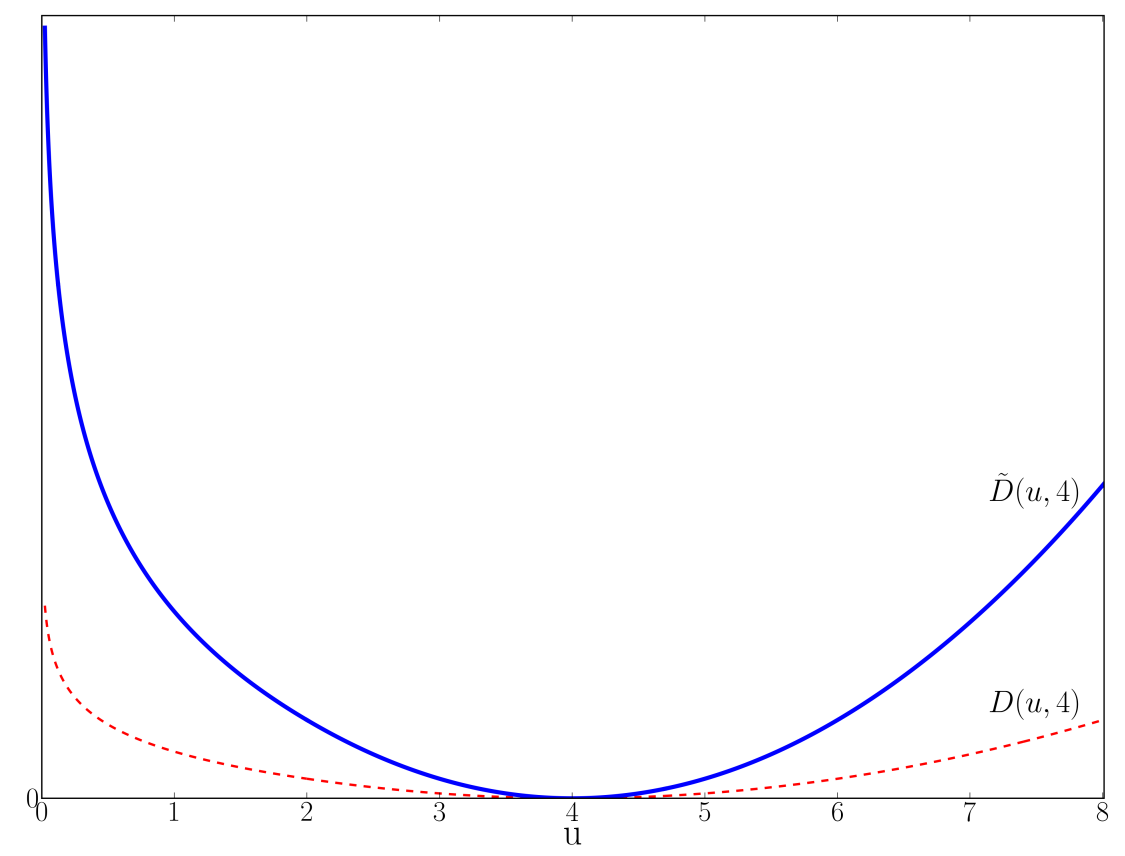

Figura 4.4: Exemplo do efeito de $w$ sobre as regularizações $D$ e $\widetilde{D}$. 
Além disso, ao elevarmos $w$, afastamos da origem os centros das distâncias $\widetilde{D}_{p}$ e $D_{p}$. Por outro lado, quanto mais distante estiver o centro de $D_{p}$, maior será sua diferença em relação a $\widetilde{D}_{p}$, tornando-se cada vez mais "achatada". Logo, um método de ponto proximal que use $D_{p}$ pode explorar pontos mais distantes do centro à medida que esses se afastam da origem. Tal efeito não é comum nos métodos tradicionais de ponto proximal, o que pode ajudar a explicar a dificuldade comentada em [MZ00] para obter-se resultados de convergência melhores para o Algoritmo (APB).

\subsection{Obtenção de limitantes duais}

Nesta seção, apresentamos limitantes duais para o valor ótimo do problema primal linear que tem a restrição adicional

$$
\|x\| \leq \rho,
$$

em que $\rho$ é um real estritamente positivo fixado. Tais limitantes são baseados na função convexo conjugada da função penalidade e podem ser úteis quando é possível obter de forma rápida um solução inexata (aproximada) do problema penalizado (4.4) ou do problema (4.7), que minimiza o lagrangiano aumentado (4.6) em relação ao seu primeiro argumento.

Os termos com papel de penalidade na funções objetivos do problema penalizado (4.4) e do problema (4.7) são

$$
\operatorname{tr}\left(\varphi_{p}\left(A_{0}+\mathcal{A}(x)\right)\right) \quad \text { e } \quad \operatorname{tr}\left(\varphi_{p}\left(V\left(A_{0}+\mathcal{A}(x)\right) V\right)\right)
$$

respectivamente. Podemos representar ambos os termos de forma equivalente por uma função convexa $\Phi: \mathbb{S}^{m} \rightarrow \mathbb{R}$, suave e de classe $C^{2}$ em que

$$
\Phi(A)=\operatorname{tr}\left(\varphi_{p}(A)\right) \quad \text { e } \quad \Phi(A)=\operatorname{tr}\left(\varphi_{p}(V A V)\right)
$$

para a função definida pela equação (4.3) e o lagrangiano aumentado (4.6), respectivamente. A partir das propriedades $(\varphi 0)$ e $(\varphi 3)$, sabemos que

$$
\Phi\left(A_{0}+\mathcal{A}(x)\right) \leq 0 \text { se } A_{0}+\mathcal{A}(x) \preceq 0 .
$$

Logo, podemos reescrever a função (4.3) e o lagrangiano aumentado (4.6) como uma função generalizada

$$
F(x) \stackrel{\text { def }}{=} c^{T} x+\Phi\left(A_{0}+\mathcal{A}(x)\right),
$$

a qual denotamos por função agregada. Obtemos, dessa forma, o seguinte problema

$$
\min _{\|x\| \leq \rho} F(x)=\min _{\|x\| \leq \rho}\left\{c^{T} x+\Phi\left(A_{0}+\mathcal{A}(x)\right)\right\},
$$

cujo valor ótimo denotamos por $\mathrm{F}_{*}^{\mathrm{P}}$.

Consideremos a função convexo conjugada de $\Phi$ :

$$
\Phi^{*}(B) \stackrel{\text { def }}{=} \sup _{A \in \operatorname{dom} \Phi}\{\langle A, B\rangle-\Phi(A)\} .
$$

Suporemos durante o restante do texto que sabemos computar $\Phi^{*}(B)$ para toda matriz $B \in$ $\operatorname{dom} \Phi^{*}$.

Exibimos a seguir um limitante inferior para o valor ótimo de (PSDL). 
Proposição 4.3.1 ([MZ00, Proposição 3]). O valor ótimo do problema (4.24) é um limitante inferior para o valor ótimo de (PSDL).

Demonstração. Já que o conjunto $\left\{x \in \mathbb{R}^{n} \mid\|x\| \leq \rho\right\}$ é compacto, sabemos que $F$ atinge um mínimo dentro desse conjunto. Logo,

$$
\begin{array}{rlr}
\mathrm{F}_{*}^{\mathrm{P}} & =\min _{\|x\| \leq \rho} F(x)=\min _{\|x\| \leq \rho}\left\{c^{T} x+\Phi\left(A_{0}+\mathcal{A}(x)\right)\right\} \\
& \leq \min _{\|x\| \leq \rho}\left\{c^{T} x+\Phi\left(A_{0}+\mathcal{A}(x)\right) \mid A_{0}+\mathcal{A}(x) \preceq 0\right\} \\
& \leq \min _{\|x\| \leq \rho}\left\{c^{T} x \mid A_{0}+\mathcal{A}(x) \preceq 0\right\} & \text { [expressão (4.23)] } \\
& =\mathrm{f}_{*}^{\mathrm{P}},
\end{array}
$$

resultando que $\mathrm{F}_{*}^{\mathrm{P}} \leq \mathrm{f}_{*}^{\mathrm{P}}$.

A partir desse resultado, podemos apresentar o seguinte teorema:

Teorema 4.3.2 ([MZ00, Teorema 1]). Seja $B \in \operatorname{dom} \Phi^{*}$ que satisfaça a equação

$$
c+\mathcal{A}^{*}(B)=\Delta,
$$

onde $\Delta \in \mathbb{R}^{n}$ é um vetor fixado e c $\in \mathbb{R}^{n}$ é o vetor fixo da função objetivo de (PSDL). Então, sob a restrição adicional (4.3), o valor

$$
f_{B} \stackrel{\text { def }}{=}\left\langle A_{0}, B\right\rangle-\Phi^{*}(B)-\rho\|\Delta\|
$$

é um limitante inferior para o valor ótimo de (PSDL).

Demonstração. Pela definição da função convexo conjugada de $\Phi$, temos

$$
\begin{aligned}
\Phi(B)^{*} & =\sup _{A \in \operatorname{dom} \Phi}\{\langle A, B\rangle-\Phi(A)\} \\
& \geq\left\langle A_{0}+\mathcal{A}(x), B\right\rangle-\Phi\left(A_{0}+\mathcal{A}(x)\right) \\
& =\left\langle A_{0}, B\right\rangle+\langle\mathcal{A}(x), B\rangle-\Phi\left(A_{0}+\mathcal{A}(x)\right) \\
& =\left\langle A_{0}, B\right\rangle+\left\langle x, \mathcal{A}^{*}(B)\right\rangle-\Phi\left(A_{0}+\mathcal{A}(x)\right) \\
& =\left\langle A_{0}, B\right\rangle+\langle x, \Delta-c\rangle-\Phi\left(A_{0}+\mathcal{A}(x)\right), \quad \text { [equação (4.25)] }
\end{aligned}
$$

para todo $x \in \mathbb{R}^{n}$, resultando que

$$
F(x)=c^{T} x+\Phi\left(A_{0}+\mathcal{A}(x)\right) \geq\left\langle A_{0}, B\right\rangle+\Delta^{T} x-\Phi(B)^{*} .
$$

Para todo $x \in \mathbb{R}^{n}$ tal que $\|x\| \leq \rho$, temos $\Delta^{T} x \geq-\rho\|\Delta\|$ a partir da desigualdade de Cauchy-Schwarz. Assim, segue da expressão (4.27) que

$$
F(x)=c^{T} x+\Phi\left(A_{0}+\mathcal{A}(x)\right) \geq\left\langle A_{0}, B\right\rangle-\rho\|\Delta\|-\Phi(B)^{*}=f_{B},
$$

ou seja, $F(x) \geq f_{B}$ para todo $x \in \mathbb{R}^{n}$ tal que $\|x\| \leq \rho$. Então, pela Proposição 4.3.1, temos

$$
\mathrm{f}_{*}^{\mathrm{P}} \geq f_{B}
$$

o que completa a demonstração. 
Este teorema nos fornece um importante resultado. Se $\hat{x} \in \mathbb{R}^{n}$ é uma solução (exata) do problema (4.24), a equação (4.25) é satisfeita se nós considerarmos $B=\Phi^{\prime}\left(A_{0}+\mathcal{A}(\hat{x})\right)$ e $\Delta=F^{\prime}(\hat{x})=0$. Logo, pelo Teorema 4.3.2, sabemos que $f_{B}=\mathrm{F}_{*}^{\mathrm{P}}=F(\hat{x})$ é um limitante dual inferior para o valor ótimo do problema primal linear. De fato, devido a $B$ pertencer a $\partial \Phi\left(A_{0}+\mathcal{A}(\hat{x})\right)$, sabemos a partir da desigualdade de Fenchel (Teorema 1.4.10) que

$$
\begin{aligned}
\Phi\left(A_{0}+\mathcal{A}(\hat{x})\right)+\Phi^{*}(B) & =\left\langle A_{0}+\mathcal{A}(\hat{x}), B\right\rangle \\
& =\left\langle A_{0}, B\right\rangle+\langle\mathcal{A}(\hat{x}), B\rangle \\
& =\left\langle A_{0}, B\right\rangle+\left\langle\hat{x}, \mathcal{A}^{*}(B)\right\rangle \\
& =\left\langle A_{0}, B\right\rangle-c^{T} \hat{x} \quad \text { [equação (4.25)]. }
\end{aligned}
$$

Já que escolhemos $B=\Phi^{\prime}\left(A_{0}+\mathcal{A}(\hat{x})\right)$, temos, pela equação acima,

$$
\left\langle A_{0}, \Phi^{\prime}\left(A_{0}+\mathcal{A}(\hat{x})\right)\right\rangle-\Phi^{*}\left(\Phi^{\prime}\left(A_{0}+\mathcal{A}(\hat{x})\right)\right)=c^{T} \hat{x}+\Phi\left(A_{0}+\mathcal{A}(\hat{x})\right) .
$$

Logo, ao usarmos essa expressão na equação (4.26), obtemos

$$
\begin{aligned}
f_{B} & =\left\langle A_{0}, B\right\rangle-\Phi^{*}(B)-\rho\|\Delta\| \\
& =\left\langle A_{0}, \Phi^{\prime}\left(A_{0}+\mathcal{A}(\hat{x})\right)\right\rangle-\Phi^{*}\left(\Phi^{\prime}\left(A_{0}+\mathcal{A}(\hat{x})\right)\right) \\
& =c^{T} \hat{x}+\Phi\left(A_{0}+\mathcal{A}(\hat{x})\right) \\
& =F(\hat{x})=\mathrm{F}_{*}^{\mathrm{P}},
\end{aligned}
$$

resultando que $f_{B}=\mathrm{F}_{*}^{\mathrm{P}}$. Por outro lado, obtemos o mesmo limitante pela Proposição 4.3.1.

Podemos melhorar esse limitante se $\Phi$ é uma das funções abordadas na expressão (4.22). Devido à propriedade $(\varphi 0)$, sabemos que $\Phi^{\prime}\left(A_{0}+\mathcal{A}(\hat{x})\right) \succeq 0$. Logo, a matriz $\Phi^{\prime}\left(A_{0}+\mathcal{A}(\hat{x})\right)$ é viável para o problema dual linear. Além disso, a partir da definição da função convexo conjugada de $\Phi$ e da propriedade $(\varphi 3)$, obtemos

$$
\Phi^{*}(B)=\sup _{A \in \operatorname{dom} \Phi}\{\langle A, B\rangle-\Phi(A)\} \geq\langle 0, B\rangle-\Phi(0)=0 .
$$

Então,

$$
\begin{aligned}
f_{B} & =\left\langle A_{0}, B\right\rangle-\Phi^{*}(B)-\rho\|\Delta\| \\
& =\left\langle A_{0}, \Phi^{\prime}\left(A_{0}+\mathcal{A}(\hat{x})\right)\right\rangle-\Phi^{*}\left(\Phi^{\prime}\left(A_{0}+\mathcal{A}(\hat{x})\right)\right) \\
& \leq\left\langle A_{0}, \Phi^{\prime}\left(A_{0}+\mathcal{A}(\hat{x})\right)\right\rangle \\
& \leq \mathrm{f}_{*}^{\mathrm{P}},
\end{aligned}
$$

em que a última desigualdade é decorrente do Teorema 2.2.1. Logo, $\left\langle A_{0}, \Phi^{\prime}\left(A_{0}+\mathcal{A}(\hat{x})\right)\right\rangle$, o valor da função objetivo de (DSDL) avaliada em $B$, é um limitante inferior do valor ótimo do problema primal melhor ou igual ao obtido por $f_{B}=\mathrm{F}_{*}^{\mathrm{P}}$.

Quando $\hat{x}$ não é uma solução exata do problema (4.24), a equação (4.25) ainda é válida para $B=\Phi^{\prime}\left(A_{0}+\mathcal{A}(\hat{x})\right)$ e $\Delta=F^{\prime}(\hat{x})$. Contudo, já que $\Delta \neq 0$, o terceiro termo do lado direito da equação (4.26) será estritamente negativo. Logo, o limitante $f_{B}$ pode ser pior do que aquele dado pelo valor ótimo do problema (4.24). 


\subsubsection{Método para calcular um limitante dual a partir de um minimizador aproximado da função agregada}

Quando $\hat{x}$ não é um minimizador exato do problema (4.24) e $B=\Phi^{\prime}\left(A_{0}+\mathcal{A}(\hat{x})\right)$, podemos projetar $B$ sobre o plano $c+\mathcal{A}^{*}(U)=0$, ou seja, obter uma matriz que é viável para (DSDL) e é a mais próxima de $B$ em relação a uma norma. Um modo razoável de executarmos tal projeção é definirmos

$$
B^{+} \stackrel{\text { def }}{=} B-\Phi^{\prime \prime}(A) \mathcal{A}\left(\left[F^{\prime \prime}(\hat{x})\right]^{-1} \Delta\right) \text {. }
$$

Assim, temos

$$
\begin{aligned}
c+\mathcal{A}^{*}\left(B^{+}\right) & =c+\mathcal{A}^{*}(B)-\mathcal{A}^{*}\left(\Phi^{\prime \prime}(A) \mathcal{A}\left(\left[F^{\prime \prime}(\hat{x})\right]^{-1} \Delta\right)\right) \\
& =c+\mathcal{A}^{*}(B)-\underbrace{\mathcal{A}^{*} \Phi^{\prime \prime}(A) \mathcal{A}}_{F^{\prime \prime}(\hat{x})}\left[F^{\prime \prime}(\hat{x})\right]^{-1} \Delta \quad \text { [linearidade de } \mathcal{A} \text { e } \mathcal{A}^{*}] \\
& =c+\mathcal{A}^{*}(B)-\Delta \\
& =c+\mathcal{A}^{*}(B)-\left(c+\mathcal{A}^{*}(B)\right) \quad[\text { Teorema 4.3.2] } \\
& =0 .
\end{aligned}
$$

Então, pelo Teorema 4.3.2, obtemos $f_{B^{+}}=\left\langle A_{0}, B^{+}\right\rangle-\Phi^{*}\left(B^{+}\right)$como um limitante dual para o valor ótimo do problema primal linear. Entretanto, devido à precisão finita das operações realizadas pelos computadores, sabemos que, na realidade, $c+\mathcal{A}^{*}\left(B^{+}\right)=\Delta^{+}$, em que $\Delta^{+}$representa o possível erro resultante de tais operações aritméticas. Assim, o limitante dual fornecido pelo Teorema 4.3.2 é

$$
f_{B^{+}}=\left\langle A_{0}, B^{+}\right\rangle-\Phi^{*}\left(B^{+}\right)-\left\|\Delta^{+}\right\| \rho .
$$




\section{Capítulo 5}

\section{Testes numéricos}

Neste capítulo, descrevemos os testes numéricos feitos com o objetivo de analisar o desempenho de um dos algoritmos abordados anteriormente neste texto. Particularmente, apresentamos os resultados de uma implementação do algoritmo de penalidade/barreira discutido no Capítulo 4.

\subsection{Apresentação da implementação feita}

Com o intuito de estudar a robustez e a eficiência de um método de lagrangiano aumentado para resolver o problema primal, nós implementamos o Algoritmo (APB) proposto por Mosheyev e Zibulevsky em [MZ00] e abordado no Capítulo 4 deste texto.

Usamos a estrutura do programa Algencan [ABMS05a, ABMS05b] como ponto de partida para a implementação do Algoritmo (APB). ALGENCAN é uma versão do algoritmo de lagrangiano aumentado Quadrático de Hestenes, Powel e Rockafellar para problemas de programação não-linear no $\mathbb{R}^{n}$ com restrições de igualdade e desigualdade. Esse método divide as restrições em dois níveis, de maneira que as restrições do nível superior são incluídas no termo de penalidade do lagrangiano aumentado enquanto que as restrições do nível inferior são tratadas explicitamente pelo método de resolução do subproblema em cada iteração do algoritmo de lagrangiano aumentado. As restrições mais comuns do nível inferior são as de caixa. Escolhemos usar o ALGENCAN como base para implementarmos o Algoritmo (APB) devido a uma sugestão feita pelo professor Ernesto G. Birgin do IME - USP, um dos desenvolvedores do projeto, e por ser um programa robusto e eficiente de lagrangianos aumentados para resolver problemas de programação não-linear. Outra justificativa é que o código do AlgENCAN é livre e aberto, o que possibilitou que estudássemos e fizéssemos modificações em sua estrutura, visto que tanto o problema abordado quanto o lagrangiano aumentado usado por (APB) são diferentes daqueles adotados pelo programa original.

AlgencAn adota o algoritmo GenCAn [BM02] para resolver os subproblemas em cada iteração do método de lagrangiano aumentado. GENCAN é um algoritmo de conjuntos ativos para a minimização de problemas de programação não-linear que possuam apenas restrições de caixa. Ele usa o método de Newton truncado baseado em gradientes conjugados para explorar as faces da caixa e o algoritmo de gradiente espectral projetado (SPG) para sair de uma face. Em nossa implementação, para que GENCAN se comportasse como um método 
de minimização irrestrita, definimos uma caixa $n$-dimensional de tamanho elevado, dada por

$$
\Omega=\left\{x \in \mathbb{R}^{n} \mid-10^{20} \leq x_{i} \leq 10^{20}, i=1, \ldots, n\right\} .
$$

Em nossos experimentos, utilizamos o programa GENCAN para resolver o subproblemas irrestritos definidos pela equação (4.7) em cada iteração do algoritmo de penalidade/barreira.

Algencan e GEncan estão codificados em FORTRAN 77, com precisão dupla, e disponíveis gratuitamente sob a licença GPL (GNU Public License) na página na Internet do projeto TANGO (http://www.ime.usp.br/ egbirgin/tango).

Para obter um melhor desempenho nas operações de álgebra linear envolvidas na implementação do algoritmo de lagrangianos aumentados, usamos as versões em FORTRAN 77 das bibliotecas LAPACK e ATLAS (Automatically Tuned Linear Algebra Software), a qual implementa uma versão otimizada das sub-rotinas da BLAS (Basic Linear Algebra Subroutines). As duas bibliotecas estão disponíveis gratuitamente sob a licença BSD (Berkeley Software Distribution) no repositório Netlib (http://www.netlib.org). Ademais, fizemos as verificações das primeiras e segundas derivadas da função lagrangiana e do lagrangiano aumentado através das sub-rotinas de derivação numérica da biblioteca de análise numérica GSL (GNU Scientific Library), escrita na linguagem C e disponível sob a licença GPL também.

\subsubsection{Parâmetros modificados nos testes}

Mantivemos a maioria do parâmetros pré-definidos do Gencan e do Algencan. Entretanto, em todas as implementações do algoritmo (APB) que fizemos, nós modificamos os seguintes parâmetros em relação aos originais estabelecidos no programa:

- hptype: essa opção define como serão computados os produtos hessiana (segunda derivada)-vetor durante a resolução dos subproblemas do algoritmo de lagrangianos aumentados. Em nossos experimentos, empregamos duas opções:

- hptype $=0$, que significa que as hessianas exatas (reais) do lagrangiano aumentado do Algoritmo (APB), da função objetivo e da restrição do problema primal serão usadas. Tais funções são fornecidas pelo usuário do Algencan.

- hptype $=9$, que significa que uma aproximação do produto da segunda derivada do lagrangiano aumentado por um vetor será feito através de quocientes incrementais. Tal opção requer uma avaliação adicional do gradiente do lagrangiano aumentado por iteração do método de gradientes conjugados executado pelo GENCAN.

- precond = 'NONE' : essa opção significa que nenhum pré-condicionamento foi empregado no método de gradientes conjugados durante a execução do GEnCAN.

- rhoauto $=$.false.: essa opção significa que o parâmetro de penalidade inicial será definido pelo usuário ao invés de ser computado automaticamente pelo programa.

- rhofrac: parâmetro entre 0 e 1 que define qual deve ser a redução mínima de inviabilidade ou de complementaridade entre duas iterações consecutivas do algoritmo 
de lagrangianos aumentados para que o parâmetro de penalidade não seja reduzido (torne o efeito da penalidade mais severo). Em nossos experimentos, empregamos rhofrac=0.8, ou seja, pedimos uma redução de inviabilidade ou complementaridade de pelo menos $20 \%$ entre duas iterações consecutivas do Algoritmo (APB).

- rhomult: parâmetro que define qual será a redução do parâmetro de penalidade caso não ocorra decréscimo de inviabilidade ou de complementaridade de pelo menos rhofrac entre duas iterações consecutivas do Algoritmo (APB). Em nossos testes, adotamos rhomult $=0.6$.

- epsfeas: define a tolerância que as condições de inviabilidade e de complementaridade devem satisfazer para que um ponto seja considerado uma solução. Em nossos testes, adotamos epsfeas $=10^{-4}$.

- epsopt: originalmente, define a tolerância que a norma infinita do gradiente projetado do lagrangiano aumentado deve satisfazer para que um ponto seja considerado ótimo durante a execução do GEnCAN. Em nossos testes, empregamos esse fator para definir a tolerância pedida na resolução exata do subproblema irrestrito. Foi adotado epsopt $=10^{-4}$ em nossos experimentos.

- maxoutit: define o número máximo de iterações do algoritmo de lagrangiano aumentado. Em nossos testes, adotamos maxoutit $=50$.

- maxtotit: define o número máximo de iterações para resolver os subproblemas do algoritmo de lagrangianos aumentados, ou seja, um limite sobre a soma de iterações em todas as execuções do Gencan. Em nossos testes, adotamos maxtotit $=1000000$.

- maxtotfc: define o número máximo de avaliações da função dos subproblemas do algoritmo de lagrangianos aumentados, ou seja, um limite sobre a soma de avaliações da função objetivo em todas as execuções do GEnCAN. Em nossos testes, adotamos maxtotfc $=5 *$ maxtotit.

Além disso, em cada iteração do algoritmo de lagrangiano aumentado, projetamos cada coordenada da matriz $V_{k}$ na caixa $\left[-10^{20}, 10^{20}\right]$. Essa estratégia procura manter limitada a matriz $U_{k}=V_{k}^{2}$ em cada iteração do método implementado da mesma forma que alguns métodos de multiplicadores para otimização não-linear procuram limitar os multiplicadores. Para evitar eventuais problemas de instabilidade númerica relacionada à função penalidade $\varphi_{p}$, o parâmetro de penalidade $p_{k}$ foi limitado inferiormente por $10^{-9}$.

\subsubsection{Escolha da função penalidade e atualização do parâmetro de pena- lidade}

Adotamos a função penalidade quadrática-logarítmica definida pela equação (4.13) na Subseção 4.1.1. Ademais, em cada iteração do Algoritmo (APB), empregamos uma estratégia para a atualização do parâmetro de penalidade $p$ que segue as idéias empregadas pelo Algencan. Se não há decréscimo suficiente das medidas de inviabilidade ou de complementaridade entre duas iterações consecutivas do algoritmo de lagrangiano aumentado, então o parâmetro de penalidade é multiplicado por rhomult. Caso contrário, o parâmetro não é alterado. 


\subsubsection{Critérios de parada}

Aceitamos um par $\left(x^{*}, U^{*}\right) \in \mathbb{R}^{n} \times \mathbb{S}^{m}$ como um par de soluções do problemas primal e dual se satisfizer as seguintes condições:

(1) viabilidade primal:

$$
\lambda_{\min }\left(\mathrm{A}_{0}+\mathcal{A}\left(\mathrm{x}^{*}\right)\right) \leq \text { epsfeas; }
$$

(2) complementaridade:

$$
\left|\left\langle\mathrm{A}_{0}+\mathcal{A}\left(\mathrm{x}^{*}\right), \mathrm{U}^{*}\right\rangle\right| \leq \text { epsfeas; }
$$

(3) a matriz $U^{*}$ ser semidefinida positiva:

$$
\lambda_{\min }\left(\mathrm{U}^{*}\right) \geq-\epsilon_{\text {MACH }}^{2 / 3}
$$

em que $\epsilon_{\text {мach }}$ é o épsilon do computador utilizado nos testes ${ }^{1}$. Tal escolha foi feita a partir de testes numéricos com o intuito de se obter uma tolerância razoável para aceitar números negativos de módulo muito pequeno como não-negativos devido aos problemas decorrentes de arredondamentos. Em nossos testes, essa tolerância foi, aproximadamente, $2.15 \times 10^{-11}$.

Observemos que tais critérios são as condições de otimalidade apresentadas no Teorema 2.3.2 e transformadas em critérios numéricos que pudessem ser implementados. É importante destacarmos que, a partir das propriedades do Algoritmo (APB), se escolhermos uma matriz $U_{0} \succ 0$, sabemos que $U_{k} \succeq 0$, para todo inteiro $k \geq 0$, de forma que a condição (3) acima não precisaria ser verificada do ponto de vista teórico. Entretanto, devido à precisão finita dos computadores, matriz $U_{k}$ pode não ser definida positiva para algum inteiro $k \geq 1$ nos testes feitos. Por tal motivo, verificamos a condição (3) em cada iteração do algoritmo de lagrangiano aumentado, interrompendo-o caso essa condição não seja satisfeita.

Além disso, limitamos em duas horas o tempo de uso do processador para a resolução de cada problema de teste, interrompendo sua execução caso ele atinja esse tempo e ainda não tenha obtido uma solução que atenda os critérios de parada. Nessa situação, consideramos que o algoritmo falhou ao resolver o problema.

\subsubsection{Ambiente de testes}

Os testes foram feitos em um computador com processador Intel ${ }^{\circledR}$ Core(TM)2 Quad Q6600 2.40GHz, 4GB de memória RAM e sistema operacional GNU Linux de 64 bits, distribuição Ubuntu 8.04.1. As implementações foram feitas a partir do ALGENCAN versão 1, cujos códigos em FORTRAN 77 foram compilados com o compilador gfortran (The GNU Fortran 95 compiler), versão 4.1.1, e as opções -04 -xf77-cpp-input foram usadas. Os códigos na linguagem $\mathrm{C}$ foram compilados com o compilador gcc (The GNU C Compiler), versão 4.1.2, e nenhuma opção de otimização foi empregada. Usamos as versões 3.6.0 e 3.0.20 das bibliotecas ATLAS e LAPACK, respectivamente, disponíveis para a distribuição GNU Linux adotada.

\footnotetext{
${ }^{1} \mathrm{Ou}$ seja, o menor número real estritamente positivo tal que $1+\epsilon_{\text {MACH }} \neq 1$.
} 


\subsection{Complexidade das principais operações}

Nesta seção, analisamos a complexidade das principais operações envolvidas na implementação do Algoritmo (APB) com base na estrutura do ALGENCAN e adoção do GENCAN para efetuar as minimizações irrestritas em cada iteração. É importante ressaltar que a complexidade estudada será em relação ao consumo de tempo (custo) para realizar uma dada operação. Tal custo geralmente está associado ao número operações aritméticas elementares (flops) feitas.

Apresentamos na Tabela 5.1 algumas operações básicas associadas a vetores e matrizes reais junto com os respectivos consumos de tempo. As referências para esses valores foram os livros de Golub e Van Loan [GVL96] e de Trefethen e Bau [TB97]. Consideramos que as matrizes envolvidas nas operações listadas na Tabela 5.1 têm dimensão $m \times m$ enquanto os vetores têm dimensão $n$, exceto na operação produto matriz-vetor, na qual o vetor tem dimensão $m$.

\begin{tabular}{|c|c|}
\hline operação & custo \\
\hline \hline produto interno de dois vetores & $O(n)$ \\
produto de duas matrizes & $O\left(\mathrm{~m}^{3}\right)$ \\
produto matriz-vetor & $O\left(\mathrm{~m}^{2}\right)$ \\
traço de uma matriz & $O(\mathrm{~m})$ \\
traço do produto de duas matrizes & $O\left(\mathrm{~m}^{2}\right)$ \\
decomposição espectral de uma matriz & $O\left(\mathrm{~m}^{3}\right)$ \\
produto de Hadamard de duas matrizes & $O\left(\mathrm{~m}^{2}\right)$ \\
\hline
\end{tabular}

Tabela 5.1: Complexidade de algumas operações de álgebra linear.

Entretanto, se as matrizes forem esparsas, então algumas operações podem ter seu consumo de tempo reduzido. Um caso particular útil é quando as matrizes possuem uma estrutura de blocos diagonais. Listamos na Tabela 5.2 as mesmas operações envolvendo matrizes apresentadas na Tabela 5.1, mas supondo que as matrizes possuem $k$ blocos diagonais com dimensões $m_{1} \times m_{1}, \ldots, m_{k} \times m_{k}$ em que $m_{1}+\cdots+m_{k}=m$. A partir dessa tabela, podemos observar que o fato de uma matriz ser diagonal por blocos pode reduzir drasticamente o tempo gasto na maioria das operações envolvidas na Tabela 5.1.

\begin{tabular}{|c|c|}
\hline operação & custo \\
\hline \hline produto de duas matrizes & $O\left(m_{1}^{3}+\cdots+m_{k}^{3}\right)$ \\
produto matriz-vetor & $O\left(m_{1}^{2}+\cdots+m_{k}^{2}\right)$ \\
traço de uma matriz & $O(m)$ \\
traço do produto de duas matrizes & $O\left(m_{1}^{2}+\cdots+m_{k}^{2}\right)$ \\
decomposição espectral de uma matriz & $O\left(m_{1}^{3}+\cdots+m_{k}^{3}\right)$ \\
produto de Hadamard de duas matrizes & $O\left(m_{1}^{2}+\cdots+m_{k}^{2}\right)$ \\
\hline
\end{tabular}

Tabela 5.2: Complexidade de algumas operações de álgebra linear para matrizes com estrutura de blocos diagonais. 
Agora, estudamos a complexidade de avaliar o lagrangiano aumentado definido pela equação (4.6), além de seu gradiente e de sua hessiana. Denotemos por $c_{\mathrm{obj}}$ o consumo de tempo para avaliar a função objetivo de (PSDA). Além disso, supomos que as avaliações da função escalar $\varphi_{p}(t)$, de sua primeira e segunda derivadas têm consumo de tempo $O(1)$. Apresentamos na Tabela 5.3 os custos de algumas operações relacionadas à avaliação do termo com função de penalidade no lagrangiano aumentado.

\begin{tabular}{|c|c|}
\hline Operação & Custo \\
\hline \hline avaliação da restrição $A_{0}+\mathcal{A}(x)$ & $O\left(\mathrm{~nm}^{2}\right)$ \\
avaliação dos produtos $V\left(A_{0}+\mathcal{A}(x)\right) V$ & $O\left(\mathrm{~m}^{3}\right)$ \\
avaliação da função matricial $\varphi_{p}\left(V\left(A_{0}+\mathcal{A}(x)\right) V\right)$ & $O\left(\mathrm{~m}^{3}\right)$ \\
cálculo do traço de $\varphi_{p}\left(V\left(A_{0}+\mathcal{A}(x)\right) V\right)$ & $O(m)$ \\
\hline
\end{tabular}

Tabela 5.3: Complexidade de operações para cômputo da penalidade do lagrangiano aumentado.

Através desses dados, vemos que o custo para avaliar o lagrangiano aumentado do Algoritmo (APB) é

$$
c_{\text {obj }}+O\left(n m^{2}+m^{3}\right) .
$$

Estudemos o custo de computar o gradiente em relação a $x$ do lagrangiano aumentado

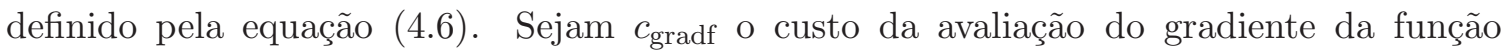
objetivo do problema primal, $V \in \mathbb{S}^{m}$ uma matriz definida positiva e $B_{i} \stackrel{\text { def }}{=} V A_{i} V \in \mathbb{S}^{m}$, para $i=0, \ldots, n$. Também definimos o operador linear $\mathcal{B}: \mathbb{R}^{n} \rightarrow \mathbb{S}^{m}$ por

$$
\mathcal{B}(x) \stackrel{\text { def }}{=} \sum_{i=1}^{n} x_{i} B_{i}=V\left(\sum_{i=1}^{n} x_{i} A_{i}\right) V=V \mathcal{A}(x) V,
$$

cujo operador adjunto é

$$
\mathcal{B}^{*}(U)=\mathcal{A}^{*}(V U V) .
$$

Logo, a equação (4.6) equivale a

$$
\mathrm{L}_{p}^{+}(x, V)=f(x)+\operatorname{tr}\left(\varphi_{p}\left(B_{0}+\mathcal{B}(x)\right)\right) .
$$

Ao aplicarmos o Corolário 1.5.6 à essa equação, obtemos

$$
\begin{aligned}
\nabla_{x} \mathrm{~L}_{p}^{+}(x, V) & =\nabla f(x)+\mathcal{B}^{*}\left(\varphi_{p}^{\prime}\left(B_{0}+\mathcal{B}(x)\right)\right) \\
& =\nabla f(x)+\mathcal{A}^{*}\left(V \varphi_{p}^{\prime}\left(V\left(A_{0}+\mathcal{A}(x)\right) V\right) V\right) .
\end{aligned}
$$

A partir da definição do operador linear $\mathcal{A}$, podemos ver que avaliar $\mathcal{A}^{*}$ consome tempo $O\left(\mathrm{~nm}^{2}\right)$. Logo, o custo de avaliar o gradiente em relação do lagrangiano aumentado é

$$
c_{\text {gradf }}+O\left(n m^{2}+m^{3}\right) \text {. }
$$


Por fim, calculamos a hessiana em relação a $x$ do lagrangiano aumentado do Algoritmo (APB) e o consumo de tempo de sua avaliação. Ao aplicarmos o Corolário 1.5.8 à equação $(5.2)$, temos

$$
\nabla_{x x}^{2} \mathrm{~L}_{p}^{+}(x, V)=\nabla^{2} f(x)+H_{F}
$$

tal que $H_{F} \in \mathbb{S}^{n}$ é uma matriz cuja $j$-ésima coluna é dada por

$$
\mathcal{B}^{*}\left(Q\left(S \circ\left(Q^{T} B_{j} Q\right)\right) Q^{T}\right)=\mathcal{A}^{*}\left(V Q\left(S \circ\left(Q^{T} V A_{j} V Q\right)\right) Q^{T} V\right),
$$

para $j=1, \ldots, n$. Na equação acima, a matriz $S=\left(s_{i j}\right) \in \mathbb{S}^{m}$ é dada por

$$
s_{i j}= \begin{cases}\frac{\varphi_{p}^{\prime}\left(\lambda_{i}\right)-\varphi_{p}^{\prime}\left(\lambda_{j}\right)}{\lambda_{i}-\lambda_{j}}, & \text { se } \lambda_{i} \neq \lambda_{j}, \\ \varphi_{p}^{\prime \prime}\left(\lambda_{i}\right), & \text { caso contrário }\end{cases}
$$

para $i, j=1, \ldots, m$. Ademais, supomos que a matriz $B_{0}+\mathcal{B}(x)$ admite uma decomposição espectral $Q \Lambda Q^{T}$, em que $\Lambda=\left(\lambda_{i j}\right) \in \mathbb{S}^{m}$. Então, ao definirmos $D \stackrel{\text { def }}{=} V Q$, concluímos pela equação (5.4) que a $j$-ésima coluna da matriz $H_{F}$ é

$$
\mathcal{A}^{*}\left(D\left(S \circ\left(D^{T} A_{j} D\right)\right) D^{T}\right)
$$

para $j=1, \ldots, n$.

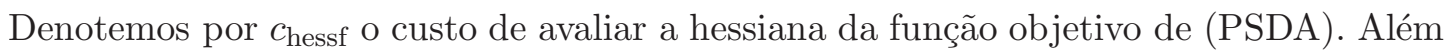
disso, há os custos listados na Tabela 5.4 para determinarmos cada coluna de $H_{F}$. Portanto, o custo de avaliar a hessiana em relação a $x$ do lagrangiano aumentado do Algoritmo (APB) é

$$
c_{\text {hessf }}+O\left(n\left(m^{3}+n m^{2}\right)\right)=c_{\text {hessf }}+O\left(n m^{3}+n^{2} m^{2}\right)
$$

\begin{tabular}{|c|c|}
\hline Operação & Custo \\
\hline \hline produto $V Q$ & $O\left(\mathrm{~m}^{3}\right)$ \\
produtos $D^{T} A_{j} D$ & $O\left(\mathrm{~m}^{3}\right)$ \\
produto de Hadamard $S \circ\left(D^{T} A_{j} D\right)$ & $O\left(\mathrm{~m}^{2}\right)$ \\
produtos $D\left(S \circ\left(D^{T} A_{j} D\right)\right) D^{T}$ & $O\left(\mathrm{~m}^{3}\right)$ \\
aplicação do operador adjunto $\mathcal{A}^{*} \operatorname{em~} D\left(S \circ\left(D^{T} A_{j} D\right)\right) D^{T}$ & $O\left(n m^{2}\right)$ \\
\hline
\end{tabular}

Tabela 5.4: Complexidade de operações para cálculo da matriz $H_{F}$.

É importante ressaltar que o algoritmo de lagrangianos aumentados (APB) não exige que a matriz de multiplicadores $U$ e nem a sua raiz quadrada $V=U^{1 / 2}$ tenham o mesmo padrão de esparsidade que as matrizes $A_{0}, A_{1}, \ldots, A_{n}$. Entretanto, conforme abordamos no início desta seção, pode haver uma grande redução no consumo de tempo do algoritmo se essas matrizes possuírem uma estrutura comum formada por blocos diagonais. Particularmente, se as matrizes $A_{0}, A_{1}, \ldots, A_{n}$ e $V$ possuírem uma mesma estrutura formada por blocos 
diagonais de mesmas dimensões que aquelas abordadas na Tabela 5.2, o custo de avaliação do lagrangiano aumentado torna-se

$$
c_{\mathrm{obj}}+O\left(n\left(m_{1}^{2}+\cdots+m_{k}^{2}\right)+m_{1}^{3}+\cdots+m_{k}^{3}\right) .
$$

Além disso, o custo de avaliação do gradiente e da hessiana do lagrangiano aumentado em relação a $x$ reduzem-se respectivamente a

$$
c_{\text {gradf }}+O\left(n\left(m_{1}^{2}+\cdots+m_{k}^{2}\right)+m_{1}^{3}+\cdots+m_{k}^{3}\right)
$$

$\mathrm{e}$

$$
c_{\text {hessf }}+O\left(n\left(m_{1}^{3}+\cdots+m_{k}^{3}\right)+n^{2}\left(m_{1}^{2}+\cdots+m_{k}^{2}\right)\right) .
$$

Exploramos tal observação posteriormente neste capítulo.

\subsection{Problemas de teste}

Selecionamos 44 problemas de teste da coleção SDPLIB [Bor99], disponível no endereço eletrônico http://www.nmt.edu/ sdplib/. Os critérios que adotamos para a escolha das instâncias foram a quantidade e a dimensão das matrizes que definem a restrição matricial do problema primal, isto é, $n$ e $m$, respectivamente. Em nossos testes, o limite para esses valores foi 250 e selecionamos apenas instâncias em que os problemas primal e dual possuíssem pontos viáveis. Apresentamos uma lista dos problemas testados na Tabela 5.5, junto com as dimensões de $n$ e $m$ e o valor ótimo reportado em [Bor99].

Todos os problemas da biblioteca SDPLIB são instâncias de (PSDL), ou seja, são casos particulares de (PSDA) em que a função objetivo é linear. Logo, a partir do que já discutimos neste capítulo, podemos concluir que $c_{\text {obj }}=O(n), c_{\text {gradf }}=O(n)$ e $c_{\text {hessf }}=O\left(n^{2}\right)$. De fato, já que $\nabla^{2} f(x)=0$, podemos considerar que $c_{\text {hessf }}=O(1)$ em alguns procedimentos. Assim, a partir das equações (5.1), (5.3) e (5.5), nós sabemos que o termo com função de penalidade no lagrangiano aumentado do Algoritmo (APB),

$$
\operatorname{tr}\left(\varphi_{p}\left(V\left(A_{0}+\mathcal{A}(x)\right) V\right)\right)
$$

domina o custo de avaliação dessa função, como também de seu gradiente e de sua hessiana em relação a $x$.

Ademais, todos os problemas da biblioteca SDPLIB estão codificados no formato esparso do programa SDPA $\left[\mathrm{FFK}^{+} 08\right]$. Nesse formato, todas as matrizes $A_{0}, A_{1}, \ldots, A_{n}$ possuem uma estrutura comum de blocos diagonais tal que cada bloco é esparso. Assim, pelo que foi abordado na seção anterior, é possível reduzir bastante o consumo de tempo em algumas operações relacionadas ao lagrangiano aumentado se adotarmos um multiplicador $V$ com o mesmo padrão de blocos diagonais que as matrizes $A_{0}, \ldots, A_{n}$. Comentamos na próxima seção os testes sobre o impacto do uso dessa estrutura por blocos diagonais.

\subsection{Resultados dos testes numéricos}

Analisamos nesta seção os resultados dos testes numéricos realizados. Empregamos perfis de desempenho (perfomance profiles) [DM02] com o objetivo de comparar as diferentes 


\begin{tabular}{|c|c|c|c||c|c|c|c|}
\hline problema & $\mathbf{n}$ & $\mathbf{m}$ & valor ótimo & problema & $\mathbf{n}$ & $\mathbf{m}$ & valor ótimo \\
\hline \hline control1 & 21 & 15 & $1.778463 \mathrm{e}+01$ & hinf10 & 21 & 18 & $1.09 \mathrm{e}+02$ \\
control2 & 66 & 30 & $8.300000 \mathrm{e}+00$ & hinf11 & 31 & 22 & $6.59 \mathrm{e}+01$ \\
control3 & 136 & 45 & $1.363327 \mathrm{e}+01$ & hinf12 & 43 & 24 & $2 \mathrm{e}-1$ \\
control4 & 231 & 60 & $1.979423 \mathrm{e}+01$ & hinf13 & 57 & 30 & $4.6 \mathrm{e}+01$ \\
gpp100 & 101 & 100 & $-4.49435 \mathrm{e}+01$ & hinf14 & 73 & 34 & $1.30 \mathrm{e}+01$ \\
gpp124-1 & 125 & 124 & $-7.3431 \mathrm{e}+00$ & hinf15 & 91 & 37 & $2.5 \mathrm{e}+01$ \\
gpp124-2 & 125 & 124 & $-4.68623 \mathrm{e}+01$ & mcp100 & 100 & 100 & $2.261574 \mathrm{e}+02$ \\
gpp124-3 & 125 & 124 & $-1.53014 \mathrm{e}+02$ & mcp124-1 & 124 & 124 & $1.419905 \mathrm{e}+02$ \\
gpp124-4 & 125 & 124 & $-4.1899 \mathrm{e}+02$ & mcp124-2 & 124 & 124 & $2.698802 \mathrm{e}+02$ \\
gpp250-1 & 250 & 250 & $-1.5445 \mathrm{e}+01$ & mcp124-3 & 124 & 124 & $4.677501 \mathrm{e}+02$ \\
gpp250-2 & 250 & 250 & $-8.1869 \mathrm{e}+01$ & mcp124-4 & 124 & 124 & $8.644119 \mathrm{e}+02$ \\
gpp250-3 & 250 & 250 & $-3.035 \mathrm{e}+02$ & mcp250-1 & 250 & 250 & $3.172643 \mathrm{e}+02$ \\
gpp250-4 & 250 & 250 & $-7.473 \mathrm{e}+02$ & mcp250-2 & 250 & 250 & $5.319301 \mathrm{e}+02$ \\
hinf1 & 13 & 14 & $2.0326 \mathrm{e}+00$ & mcp250-3 & 250 & 250 & $9.811726 \mathrm{e}+02$ \\
hinf2 & 13 & 16 & $1.0967 \mathrm{e}+01$ & mcp250-4 & 250 & 250 & $1.681960 \mathrm{e}+03$ \\
hinf3 & 13 & 16 & $5.69 \mathrm{e}+01$ & qap5 & 136 & 26 & $-4.360 \mathrm{e}+02$ \\
hinf4 & 13 & 16 & $2.74764 \mathrm{e}+02$ & qap6 & 229 & 37 & $-3.8144 \mathrm{e}+02$ \\
hinf5 & 13 & 16 & $3.63 \mathrm{e}+02$ & theta1 & 104 & 50 & $2.300000 \mathrm{e}+01$ \\
hinf6 & 13 & 16 & $4.490 \mathrm{e}+02$ & truss1 & 6 & 13 & $-8.999996 \mathrm{e}+00$ \\
hinf7 & 13 & 16 & $3.91 \mathrm{e}+02$ & truss2 & 58 & 133 & $-1.233804 \mathrm{e}+02$ \\
hinf8 & 13 & 16 & $1.16 \mathrm{e}+02$ & truss3 & 27 & 31 & $-9.109996 \mathrm{e}+00$ \\
hinf9 & 13 & 16 & $2.3625 \mathrm{e}+02$ & truss4 & 12 & 19 & $-9.009996 \mathrm{e}+00$ \\
\hline
\end{tabular}

Tabela 5.5: problemas da biblioteca SDPLIB utilizados nos testes.

implementações feitas. Um medidor de desempenho para um algoritmo é a distribuição cumulativa de uma medida de interesse selecionada, em que são comparados o desempenho de um dado algoritmo para resolver um problema com o desempenho do melhor algoritmo que resolveu tal problema, de maneira que é possível saber em quantos problemas cada algoritmo é o melhor e quanto tempo adicional em relação ao tempo do melhor algoritmo é necessário para que um deles resolva o mesmo problema. A adoção de tais perfis possibilita comparar simultaneamente a eficiência e a robustez de diferentes algoritmos para resolver um conjunto de problemas considerando-se uma dada medida, mesmo que um deles falhe ao resolver algum problema, e evita-se que um pequeno número de problemas difíceis domine a análise.

Usamos o número de iterações internas realizadas pelo GENCAN como medida de desempenho da implementação. Tal escolha deve-se ao fato de que a resolução do subproblema irrestrito é a operação que domina o consumo de tempo em cada iteração do algoritmo de lagrangiano aumentado. De fato, em cada iteração do GENCAN, de acordo com a estratégia selecionada para o produto hessiana-vetor, é feita uma avaliação da hessiana ou várias avaliações do gradiente do lagrangiano aumentado e, conforme discutimos na Seção 5.2, essas operações estão entre as mais custosas envolvidas no Algoritmo (APB).

Apresentamos a seguir alguns testes feitos com o objetivo de esclarecer as decisões 
tomadas sobre o ajuste de parâmetros e sobre o uso da estrutura de blocos diagonais.

\subsubsection{Escolha dos pontos iniciais primal e dual}

Vimos no Capítulo 4 que a seqüência de multiplicadores $\left\{U_{k}\right\}$ gerada pelo Algoritmo (APB) é a mesma obtida por um algoritmo de ponto proximal aplicado sobre o problema dual e que a seqüência $\left\{x^{k}\right\}$ é gerada a partir dessa seqüência de matrizes. Logo, a escolha do ponto primal não tem muita importância sobre o desempenho do Algoritmo (APB). De fato, mesmo que escolhêssemos uma solução do problema primal como o ponto inicial $x^{0}$ mas um ponto dual inicial $U_{0}$ que fosse diferente da solução dual associada, teríamos que efetuar pelo menos uma iteração do Algoritmo (APB). Por tal motivo, escolhemos o ponto inicial $x^{0}=0$.

Por outro lado, a escolha do ponto dual inicial, o multiplicador $U_{0}$, tem grande influência na obtenção de um par de soluções primal e dual. Em nossos experimentos, escolhemos $U_{0}=\gamma^{2} I_{m}$, em que $\gamma$ é um real estritamente positivo. Vemos facilmente que $U_{0}$ é um ponto viável do problema dual e que $V_{0}=U_{0}^{1 / 2}=\gamma I_{m}$. A escolha desse ponto inicial do problema dual foi motivada pelo fato de não termos nenhuma informação prévia sobre as soluções do problema dual e de que o uso de uma matriz com estrutura simples pode reduzir drasticamente o consumo de tempo na minimização irrestrita do primeiro subproblema do algoritmo de lagrangianos aumentados. De fato, por $U_{0}$ ser uma matriz diagonal, trivialmente, ela possui a mesma estrutura de blocos diagonais que as matrizes $A_{0}, A_{1}, \ldots, A_{n}$. Dessa forma, os consumos de tempo fornecidos pelas equações (5.6), (5.7) e (5.8) valem na primeira iteração do método implementado, implicando em uma redução no consumo de tempo para avaliar o lagrangiano aumentado, sua derivada e sua hessiana na primeira iteração do Algoritmo (APB).

Com o intuito de analisarmos a redução de tempo obtida a partir do uso de um múltiplo da matriz identidade como um multiplicador inicial, comparamos o tempo gasto na resolução do primeiro subproblema do Algoritmo (APB) por uma estratégia que aproveita o fato de $U_{0}$ ser um múltiplo da identidade com o gasto por aquela que trata $U_{0}$ como uma matriz simétrica qualquer. Denotamos a primeira alternativa por implementacao-alt-ident e a última por implementacao-pura. Como podemos ver a partir da Tabela 5.6, houve redução no tempo total gasto para resolver o primeiro subproblema em 39 dos 44 problemas de teste. Tal resultado fica ainda mais evidente ao analisarmos o perfil de desempenho da Figura 5.1. Embora possamos observar pela Tabela 5.6 e pela Figura 5.2 que há um pequeno aumento no número de iterações realizadas para resolver cada instância de teste, tal acréscimo é compensado pela redução de tempo de implementacao-alt-ident. Por essa razão, em todos testes seguintes, usamos o fato do ponto inicial do problema dual ser um múltiplo da identidade para resolver o subproblema na primeira iteração do Algoritmo (APB).

Vamos explicar agora qual valores de $\gamma$ foram testados para definir a matriz de multiplicadores inicial. Ao tomarmos $V=U^{1 / 2}=\gamma I$, o lagrangiano aumentado introduzido na 


\begin{tabular}{|c|c|c|c|c|c|c|c|}
\hline \multicolumn{4}{|c|}{ implementacao-pura } & \multicolumn{4}{|c|}{ implementacao-alt-ident } \\
\hline problema & $\begin{array}{c}\text { tempo } \\
\text { total(s) }\end{array}$ & $\begin{array}{c}\text { tempo } \\
\text { médio(s) }\end{array}$ & iterações & problema & $\begin{array}{c}\text { tempo } \\
\text { total }(\mathrm{s})\end{array}$ & $\begin{array}{c}\text { tempo } \\
\text { médio(s) }\end{array}$ & iterações \\
\hline control1 & $1.0401 \mathrm{e}-01$ & 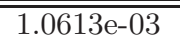 & 98 & control1 & $\overline{77.2004 \mathrm{e}-02}$ & (6.6670e-04 & 108 \\
\hline control2 & $1.8281 \mathrm{e}+00$ & $1.3955 \mathrm{e}-02$ & 131 & control2 & $1.2761 \mathrm{e}+00$ & $9.1149 \mathrm{e}-03$ & 140 \\
\hline control3 & $1.5601 \mathrm{e}+01$ & $1.0686 \mathrm{e}-01$ & 146 & control3 & $1.1009 \mathrm{e}+01$ & $7.1485 \mathrm{e}-02$ & 154 \\
\hline control4 & $1.2011 \mathrm{e}+02$ & $5.2449 \mathrm{e}-01$ & 229 & control4 & $9.4994 \mathrm{e}+01$ & $4.3575 \mathrm{e}-01$ & 218 \\
\hline gpp100 & $1.8317 \mathrm{e}+01$ & $6.3163 \mathrm{e}-01$ & 29 & gpp100 & $1.1173 \mathrm{e}+02$ & $5.3203 \mathrm{e}-01$ & 210 \\
\hline gpp124-1 & $3.2522 \mathrm{e}+01$ & $1.2508 \mathrm{e}+00$ & 26 & gpp124-1 & $1.7525 \mathrm{e}+01$ & $1.0309 \mathrm{e}+00$ & 17 \\
\hline gpp124-2 & $3.5055 \mathrm{e}+02$ & $1.2655 \mathrm{e}+00$ & 277 & gpp124-2 & $3.0354 \mathrm{e}+02$ & $1.0360 \mathrm{e}+00$ & 293 \\
\hline gpp124-3 & $1.7949 \mathrm{e}+01$ & $1.2821 \mathrm{e}+00$ & 14 & gpp124-3 & $1.1605 \mathrm{e}+01$ & $1.0550 \mathrm{e}+00$ & 11 \\
\hline gpp124-4 & $1.6989 \mathrm{e}+01$ & $1.3069 \mathrm{e}+00$ & 13 & gpp124-4 & $1.3621 \mathrm{e}+01$ & $1.0478 \mathrm{e}+00$ & 13 \\
\hline gpp250-1 & $4.7133 \mathrm{e}+02$ & $1.6833 \mathrm{e}+01$ & 28 & gpp250-1 & $3.5999 \mathrm{e}+02$ & $1.3846 \mathrm{e}+01$ & 26 \\
\hline gpp250-2 & $3.2428 \mathrm{e}+02$ & $1.7068 \mathrm{e}+01$ & 19 & gpp250-2 & $2.6168 \mathrm{e}+02$ & $1.3773 \mathrm{e}+01$ & 19 \\
\hline gpp250-3 & $2.0642 \mathrm{e}+02$ & $1.7201 \mathrm{e}+01$ & 12 & gpp250-3 & $1.8101 \mathrm{e}+02$ & $1.3924 \mathrm{e}+01$ & 13 \\
\hline gpp250-4 & $2.2185 \mathrm{e}+02$ & $1.7065 \mathrm{e}+01$ & 13 & gpp250-4 & $1.9343 \mathrm{e}+02$ & $1.3816 \mathrm{e}+01$ & 14 \\
\hline hinf1 & $5.6003 \mathrm{e}-02$ & $7.0004 \mathrm{e}-04$ & 80 & hinf1 & $1.6001 \mathrm{e}-02$ & $5.5176 \mathrm{e}-04$ & 29 \\
\hline hinf2 & $1.8001 \mathrm{e}-01$ & $8.9114 \mathrm{e}-04$ & 202 & hinf2 & $8.4005 \mathrm{e}-02$ & $3.6524 \mathrm{e}-04$ & 230 \\
\hline hinf3 & $1.5601 \mathrm{e}-01$ & $8.4330 \mathrm{e}-04$ & 185 & hinf3 & $1.3601 \mathrm{e}-01$ & $5.1131 \mathrm{e}-04$ & 266 \\
\hline $\operatorname{hinf} 4$ & $1.1601 \mathrm{e}-01$ & $8.9928 \mathrm{e}-04$ & 129 & $\operatorname{hinf} 4$ & $6.4004 \mathrm{e}-02$ & $4.7062 \mathrm{e}-04$ & 136 \\
\hline hinf5 & $3.4802 \mathrm{e}-01$ & $8.2665 \mathrm{e}-04$ & 421 & hinf5 & $1.5201 \mathrm{e}-01$ & $3.9483 \mathrm{e}-04$ & 385 \\
\hline hinf6 & $1.5681 \mathrm{e}+00$ & $7.7667 \mathrm{e}-04$ & 2019 & hinf6 & $7.5205 \mathrm{e}-01$ & $3.8292 \mathrm{e}-04$ & 1964 \\
\hline $\operatorname{hinf7}$ & $2.6202 \mathrm{e}+00$ & $7.8921 \mathrm{e}-04$ & 3320 & $\operatorname{hinf7}$ & $1.6441 \mathrm{e}+00$ & $3.9617 \mathrm{e}-04$ & 4150 \\
\hline hinf8 & $4.6003 \mathrm{e}-01$ & $7.5046 \mathrm{e}-04$ & 613 & hinf8 & $2.1201 \mathrm{e}-01$ & $4.5890 \mathrm{e}-04$ & 462 \\
\hline hinf9 & $1.6241 \mathrm{e}+00$ & $8.3032 \mathrm{e}-04$ & 1956 & hinf9 & $8.3205 \mathrm{e}-01$ & $4.3179 \mathrm{e}-04$ & 1927 \\
\hline hinf10 & $4.9603 \mathrm{e}-01$ & $1.4896 \mathrm{e}-03$ & 333 & hinf10 & $2.4802 \mathrm{e}-01$ & $8.1051 \mathrm{e}-04$ & 306 \\
\hline hinf11 & $1.1801 \mathrm{e}+00$ & $3.1553 \mathrm{e}-03$ & 374 & hinf11 & $5.4803 \mathrm{e}-01$ & $1.4575 \mathrm{e}-03$ & 376 \\
\hline hinf12 & $1.9561 \mathrm{e}+00$ & $5.5258 \mathrm{e}-03$ & 354 & hinf12 & $9.9206 \mathrm{e}-01$ & $2.8345 \mathrm{e}-03$ & 350 \\
\hline hinf13 & $1.3945 \mathrm{e}+01$ & $1.1468 \mathrm{e}-02$ & 1216 & hinf13 & $7.2645 \mathrm{e}+00$ & $5.9642 \mathrm{e}-03$ & 1218 \\
\hline hinf14 & $1.5285 \mathrm{e}+01$ & $2.0245 \mathrm{e}-02$ & 755 & hinf14 & $2.4326 \mathrm{e}+01$ & $1.0549 \mathrm{e}-02$ & 2306 \\
\hline hinf15 & $1.6491 \mathrm{e}+02$ & $3.2982 \mathrm{e}-02$ & 5000 & hinf15 & $8.7769 \mathrm{e}+01$ & $1.7554 \mathrm{e}-02$ & 5000 \\
\hline mcp100 & $1.3593 \mathrm{e}+01$ & $6.1786 \mathrm{e}-01$ & 22 & mcp100 & $1.2145 \mathrm{e}+01$ & $5.2803 \mathrm{e}-01$ & 23 \\
\hline mcp124-1 & $2.7018 \mathrm{e}+01$ & $1.2281 \mathrm{e}+00$ & 22 & mcp124-1 & $2.7814 \mathrm{e}+01$ & $9.9335 \mathrm{e}-01$ & 28 \\
\hline mcp124-2 & $3.2198 \mathrm{e}+01$ & $1.2384 \mathrm{e}+00$ & 26 & mcp124-2 & $2.6614 \mathrm{e}+01$ & $1.0236 \mathrm{e}+00$ & 26 \\
\hline mcp124-3 & $3.0506 \mathrm{e}+01$ & $1.2202 \mathrm{e}+00$ & 25 & mcp124-3 & $2.5050 \mathrm{e}+01$ & $1.0020 \mathrm{e}+00$ & 25 \\
\hline mcp124-4 & $2.6490 \mathrm{e}+01$ & $1.2614 \mathrm{e}+00$ & 21 & mcp124-4 & $2.1421 \mathrm{e}+01$ & $1.0201 \mathrm{e}+00$ & 21 \\
\hline mcp250-1 & $4.8473 \mathrm{e}+02$ & $1.6715 \mathrm{e}+01$ & 29 & mcp250-1 & $3.9465 \mathrm{e}+02$ & $1.3609 \mathrm{e}+01$ & 29 \\
\hline mcp250-2 & $4.3192 \mathrm{e}+02$ & $1.6612 \mathrm{e}+01$ & 26 & mcp250-2 & $3.5354 \mathrm{e}+02$ & $1.3598 \mathrm{e}+01$ & 26 \\
\hline mcp250-3 & $4.3426 \mathrm{e}+02$ & $1.6702 \mathrm{e}+01$ & 26 & mcp250-3 & $3.5257 \mathrm{e}+02$ & $1.3560 \mathrm{e}+01$ & 26 \\
\hline mcp250-4 & $3.1890 \mathrm{e}+02$ & $1.6784 \mathrm{e}+01$ & 19 & mcp250-4 & $2.5817 \mathrm{e}+02$ & $1.3588 \mathrm{e}+01$ & 19 \\
\hline qap5 & $7.6753 \mathrm{e}+01$ & $4.4340 \mathrm{e}-02$ & 1731 & qap5 & $1.1324 \mathrm{e}+02$ & $4.2911 \mathrm{e}-02$ & 2639 \\
\hline qap6 & $1.5034 \mathrm{e}+03$ & $3.0068 \mathrm{e}-01$ & 5000 & qap6 & $1.4522 \mathrm{e}+03$ & $2.9043 \mathrm{e}-01$ & 5000 \\
\hline theta1 & $3.2442 \mathrm{e}+00$ & $1.2977 \mathrm{e}-01$ & 25 & theta1 & $3.5842 \mathrm{e}+00$ & $1.1562 \mathrm{e}-01$ & 31 \\
\hline truss1 & $1.2001 \mathrm{e}-02$ & $3.4289 \mathrm{e}-04$ & 35 & truss1 & $8.0010 \mathrm{e}-03$ & $2.2860 \mathrm{e}-04$ & 35 \\
\hline truss2 & $2.5518 \mathrm{e}+01$ & $2.7438 \mathrm{e}-01$ & 93 & truss2 & $1.3921 \mathrm{e}+00$ & $1.4809 \mathrm{e}-02$ & 94 \\
\hline truss3 & $1.6401 \mathrm{e}-01$ & $5.2906 \mathrm{e}-03$ & 31 & truss 3 & $5.2003 \mathrm{e}-02$ & $1.6775 \mathrm{e}-03$ & 31 \\
\hline truss 4 & $4.0002 \mathrm{e}-02$ & $1.1765 \mathrm{e}-03$ & 34 & truss 4 & $1.2000 \mathrm{e}-02$ & $3.5294 \mathrm{e}-04$ & 34 \\
\hline
\end{tabular}

Tabela 5.6: comparação entre uma estratégia que usa explicitamente o fato de $U_{0}$ ser um múltiplo da identidade e outra que trata essa matriz como uma matriz simétrica qualquer ao resolver o primeiro subproblema do algoritmo de lagrangianos aumentados. 


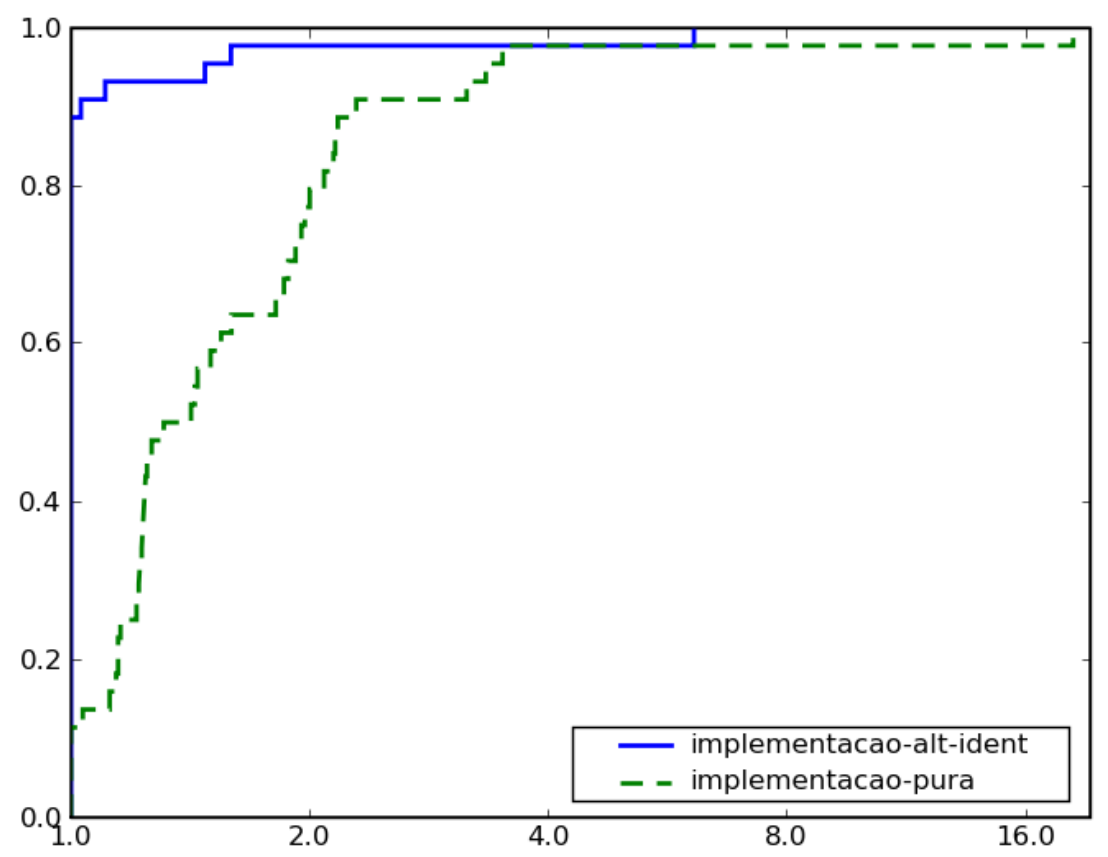

Figura 5.1: Tempo total por instância para resolver o primeiro subproblema do Algoritmo (APB).

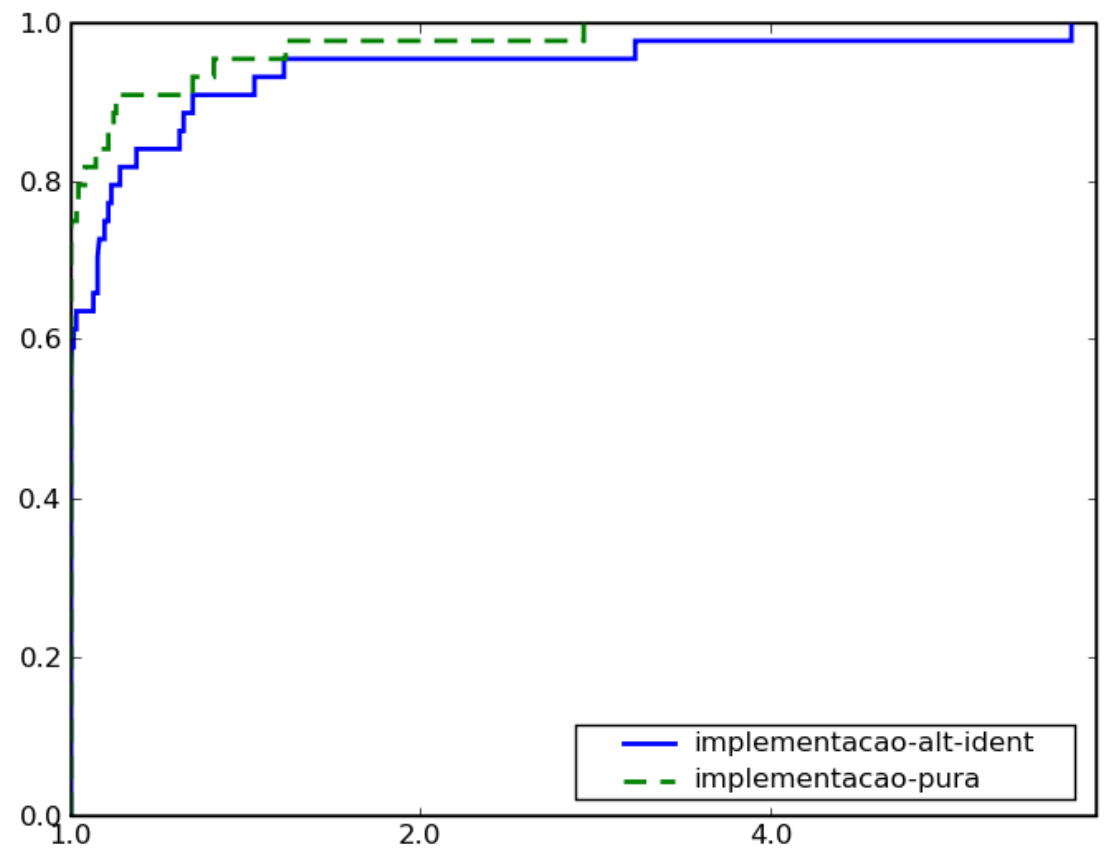

Figura 5.2: Quantidade de iterações do GENCAN por instância para resolver o primeiro subproblema do Algoritmo (APB). 
equação (4.6) torna-se

$$
\begin{aligned}
\mathrm{L}_{p}^{+}(x, V) & =f(x)+\operatorname{tr}\left(\varphi_{p}\left(\gamma I\left(A_{0}+\mathcal{A}(x)\right) \gamma I\right)\right) \\
& =f(x)+\operatorname{tr}\left(\varphi_{p}\left(\gamma^{2}\left(A_{0}+\mathcal{A}(x)\right)\right)\right),
\end{aligned}
$$

isto é, nós aplicamos a função penalidade $\varphi_{p}$ sobre a restrição do problema primal multiplicada por $\gamma^{2}$, implicando que seus autovalores são multiplicados por $\gamma^{2}$. Logo, pela definição da função penalidade $\varphi$ feita no capítulo 4 , a escolha de $\gamma>1$ na primeira iteração do Algoritmo (APB) impõe uma penalidade mais severa para pontos inviáveis do problema primal. Vale ressaltar que, como discutimos na Subseção 4.2.2, o papel do multiplicador como um parâmetro da função penalidade não é comum nos métodos usuais de lagrangianos aumentados.

Por outro lado, ao estudarmos o problema dual, a regularização do método de ponto proximal é

$$
\begin{aligned}
D_{p}\left(U, \gamma^{2} I\right) & =\operatorname{tr} \varphi_{p}^{*}\left(\gamma^{-1} I U \gamma^{-1} I\right)=\operatorname{tr} \varphi_{p}^{*}\left(\gamma^{-2} U\right) \\
& =\sum_{i=1}^{m} \varphi_{p}^{*}\left(\lambda_{i}\left(\gamma^{-2} U\right)\right)=\sum_{i=1}^{m} \varphi_{p}^{*}\left(\gamma^{-2} \lambda_{i}(U)\right) \\
& =p \sum_{i=1}^{m} \varphi^{*}\left(\gamma^{-2} \lambda_{i}(U)\right), \quad \text { [Proposição 4.2.1] }
\end{aligned}
$$

onde o mínimo é atingido em $\gamma^{2} I$.

Testamos três escolhas do parâmetro $\gamma$ :

- $\gamma=1$ : essa é a escolha usual, resultando em $U_{0}=I$. A partir das expressões (5.9) e (5.10), vemos que $\gamma=1$ implica em uma baixa penalidade à violação da restrição do problema primal e uma barreira $\varphi^{*}$ cujo mínimo é 1 . Denotamos por exato-1 a implementação que adota essa escolha de $\gamma$.

- $\gamma=80$ : a partir desta escolha, temos $U_{0}=6.4 \times 10^{3} I$, implicando que impomos uma multa considerável à violação da restrição matricial do problema primal. Denotamos essa implementação por exato-6400.

- $\gamma=10^{3}$ : para essa escolha, obtemos $U_{0}=10^{6} \mathrm{I}$, implicando que impomos uma multa muito alta à violação da restrição do problema primal e fixamos no ponto $10^{6} \mathrm{o}$ mínimo da barreira $\varphi^{*}$. Tal escolha de $\gamma$, embora elevada, não acarretou em problemas de instabilidade numérica. Denotamos por exato-10^ 6 a implementação que adota esse valor de $\gamma$.

Apresentamos no Gráfico 5.3 os resultados de testes feitos com essas três escolhas do parâmetro $\gamma$. Como podemos observar a partir desse gráfico, a estratégia exato-10^ 6 é mais robusta que as demais, resolvendo aproximadamente $86.4 \%$ dos problemas (38), embora seja uma das mais rápidas em menos de 30\% deles. Já a estratégia exato-6400 é uma das mais eficientes em um conjunto maior de problemas, em torno de $40 \%$ deles. Finalmente, a estratégia exato-1 apresenta um desempenho muito inferior às demais, tanto 
em robustez, resolvendo menos de $40 \%$ dos problemas, quanto em eficiência, sendo uma das estratégias mais rápidas somente em $20 \%$ deles aproximadamente. Para evidenciar as diferenças de desempenho entre as implementações exato-10^ 6 e exato-6400, comparamos as duas no Gráfico 5.4, no qual podemos confirmar que exato-6400 é mais rápida que exato-10^ 6 , embora seja menos robusta que a segunda. Contudo, tal diferença de eficiência entre as duas estratégias não é muito grande pois, com menos de duas vezes o tempo da implementação mais rápida para resolver cada problema, a estratégia exato-10^ 6 já é mais robusta que exato-6400.

Pelo que abordamos na Subseção 4.2.2, conforme aumentamos $\gamma$, o centro da distância $D$ é afastado da borda do cone $\mathbb{S}_{+}^{m}$ e, ao mesmo tempo, tal regularização tem um atuação mais suave no método de ponto proximal, possibilitando que ele procure por pontos mais distantes de seu ponto inicial, o centro de $D$. Essa situação pode aumentar as chances do método encontrar uma solução do problema dual, mas também pode resultar em mais iterações, o que condiz com os resultados dos Gráficos 5.3 e 5.4, que indicam um maior robustez de exato-10^ 6 combinada à pequena perda de eficiência.

Devido à maior robustez apresentada, adotamos exato-10^ 6 como padrão para definir o multiplicador inicial $U_{0}$ do Algoritmo (APB) nos próximos testes deste capítulo, o qual denotamos apenas por exato para simplificar a notação.

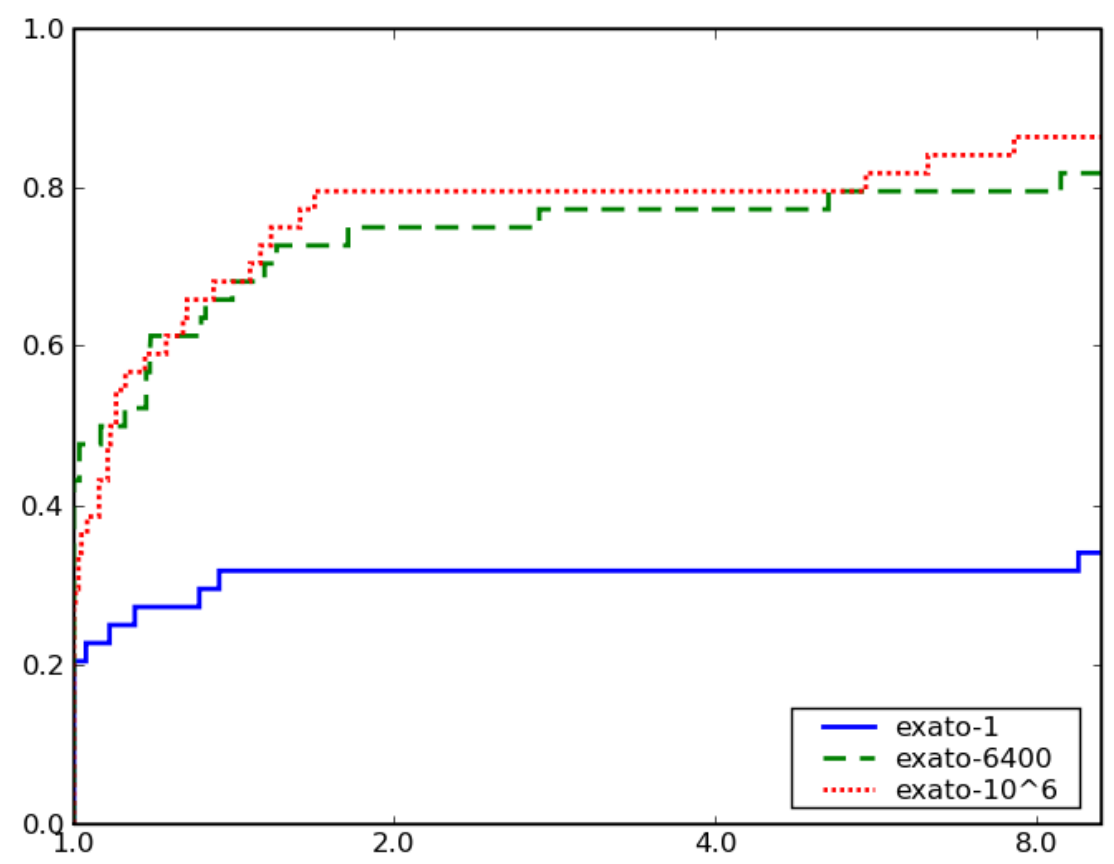

Figura 5.3: Comparação entre três escolhas do multiplicador inicial $U_{0}$ do Algoritmo (APB).

\subsubsection{Comparação entre o uso da hessiana real e de sua aproximação por quocientes incrementais no produto matriz-vetor}

Conforme mencionamos no início deste capítulo, a versão padrão do GENCAN emprega o método de Newton truncado baseado em gradientes conjugados para encontrar uma possível 


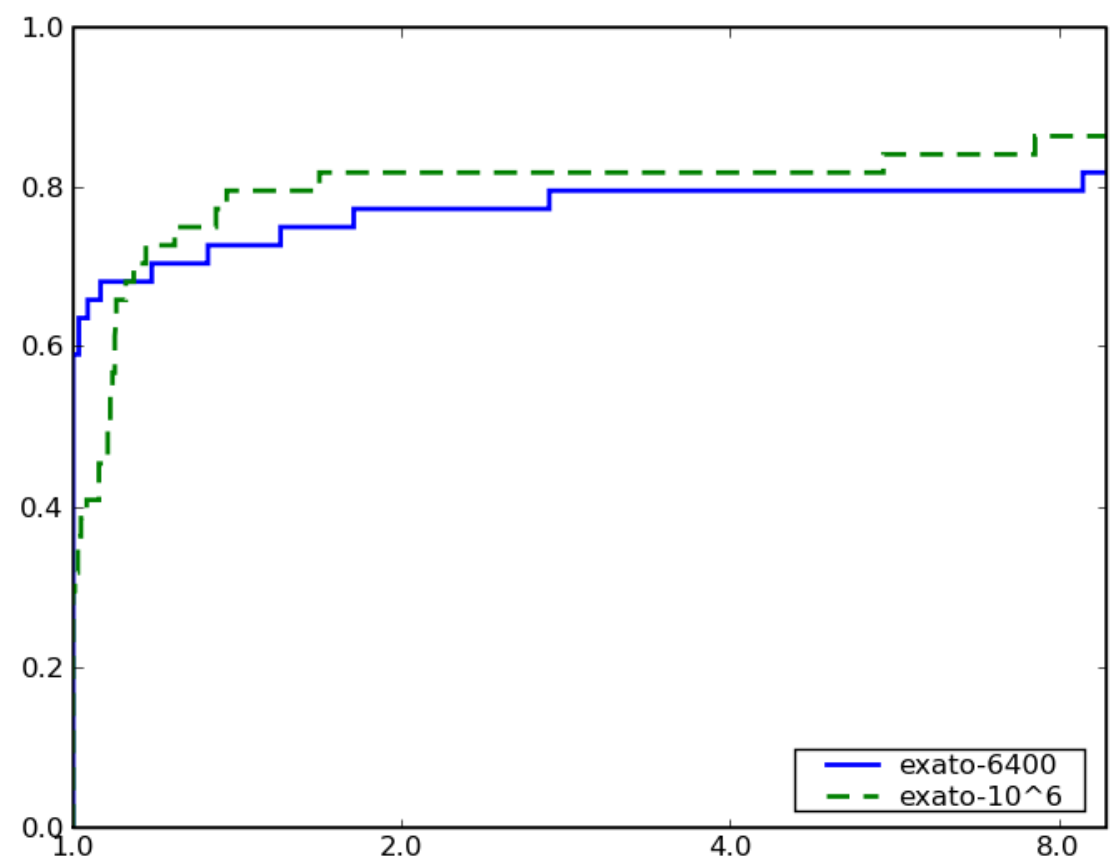

Figura 5.4: Comparação entre as duas escolhas do multiplicador inicial $U_{0}$ Algoritmo (APB) com melhor desempenho.

direção de descida em cada iteração. A partir desse fato, decidimos estudar se o uso da hessiana do lagrangiano aumentado do Algoritmo (APB) tem um desempenho superior à utilização de uma aproximação do produto hessiana-vetor por quocientes incrementais em cada passo do método de gradientes conjugados. A justificativa desse estudo decorre da análise de complexidade que fizemos na Seção 5.2. A partir da equação (5.5), sabemos que o consumo de tempo da avaliação da hessiana do lagrangiano aumentado é $O\left(\mathrm{~nm}^{3}+\right.$ $\left.n^{2} m^{2}\right)$. Logo, para valores razoáveis de $n$ e $m$, pode ser extremamente custoso calcular essa matriz. Por outro lado, ao compararmos as equações (5.3) e (5.5), vemos que a avaliação da hessiana do lagrangiano aumentado consome da ordem de $n$ vezes mais tempo que a avaliação do gradiente. Dessa forma, já que estamos interessados no produto hessiana-vetor e não propriamente na hessiana no método de gradientes conjugados, pode ser interessante avaliar esse produto por meio de quocientes incrementais. Assim, para todo $y \in \mathbb{R}^{m}$, podemos computar

$$
\nabla_{x x}^{2} \mathrm{~L}_{p}^{+}(x, V) y=\frac{\nabla_{x} \mathrm{~L}_{p}^{+}(x+t y, V)-\nabla_{x} \mathrm{~L}_{p}^{+}(x, V)}{t},
$$

em que $t$ é um real estritamente positivo de módulo pequeno. Vale destacar que, ao usarmos quocientes incrementais, é feita uma avaliação do gradiente do lagrangiano aumentado em cada passo do método de gradientes conjugados além de mais uma avaliação no início de cada iteração do Gencan. Por outro lado, ao utilizarmos a hessiana do lagrangiano aumentado, é feita uma única avaliação da hessiana em cada iteração do GENCAN e um produto matriz-vetor em cada passo do método de gradientes conjugados.

Denotamos por qincr a estratégia que usa quocientes incrementais para computar o produto hessiana-vetor e comparamos seu desempenho com a estratégia exato no Gráfico 5.5. 
A partir da análise do gráfico, podemos concluir que o uso da hessiana real para o cálculo do produto hessiana-vetor é muito mais eficiente e robusto para resolver os problemas de testes do que a utilização da aproximação por quocientes incrementais. De fato, a estratégia qincr é uma das mais rápidas em menos de $20 \%$ dos problemas e resolve aproximadamente $40 \%$ deles, enquanto a estratégia exato-10^ 6 é uma das mais rápidas em mais de $80 \%$ e resolve mais de $80 \%$. Por essa justificativa, vamos adotar a primeira estratégia em todos os testes seguintes deste capítulo.

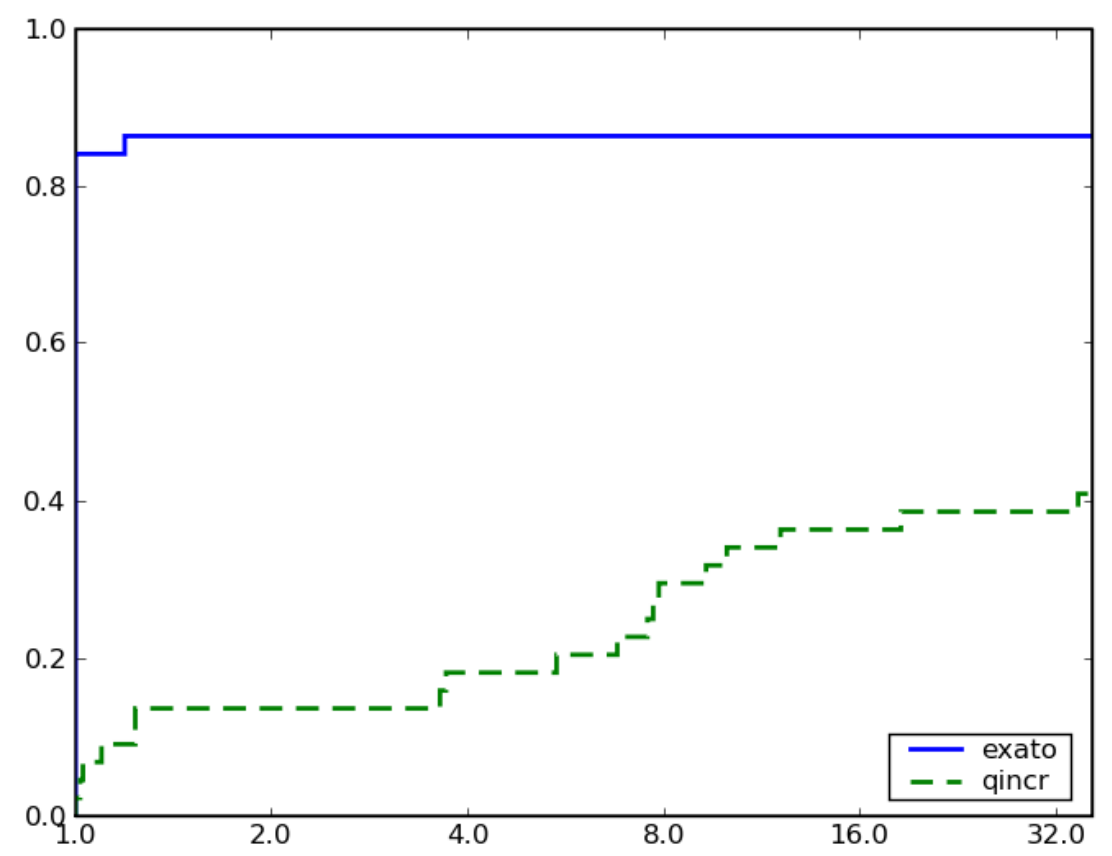

Figura 5.5: Comparação entre o uso da hessiana real e de sua aproximação por quocientes incrementais durante a execução do GENCAN.

\subsubsection{Resolução aproximada dos subproblemas do algoritmo de lagrangi- anos aumentados}

Durante os testes numéricos, em cada iteração $k$ do Algoritmo (APB), consideramos que a minimização do lagrangiano aumentado foi exata se

$$
\left\|\nabla_{x} \mathrm{~L}_{p_{k}}^{+}\left(x^{*}, V_{k}\right)\right\| \leq \text { epsopt. }
$$

Entretanto, pedir tal precisão em todas as iterações do algoritmo pode ser extremamente custoso, aumentando consideravelmente o tempo gasto para resolver um problema. Logo, a obtenção de soluções "aproximadas" do subproblemas do algoritmo de lagrangiano aumentado quando se está longe de um par de soluções dos problemas primal e dual pode ser uma opção interessante. Tal abordagem pode gerar uma redução no tempo gasto para a resolução dos subproblemas do algoritmo de lagrangianos aumentados, em particular no número de iterações do GENCAN, o que implica em uma redução na quantidade de avaliações do produto hessiana-vetor. 
Todavia, precisamos que a solução aproximada fornecida na minimização irrestrita não seja muito grosseira em relação à solução exata, pois tal situação pode aumentar muito o número de iterações externas do Algoritmo (APB) e, além disso, talvez não gere uma seqüência que convirja para um par de soluções dos problemas primal e dual. A idéia de aumentar gradativamente a precisão pedida para as soluções da subproblemas de um algoritmo do lagrangiano aumentado já é conhecida há bastante tempo. As principais referências estudadas sobre esse assunto foram dois livros de Bertsekas [Ber82, Ber99].

Fizemos testes com duas variações para a obtenção de uma solução aproximada dos subproblemas e as comparamos com a minimização exata. Na primeira alternativa, visto que a escolha do ponto inicial do problema dual foi arbitrária, pois não possuímos nenhum conhecimento prévio sobre suas soluções, torna-se razoável pensar em obter uma solução aproximada para o subproblema da primeira iteração do algoritmo de lagrangianos aumentados. A partir da segunda iteração do Algoritmo (APB), já utilizamos a precisão epsopt, segundo a qual consideramos uma solução como "exata". Em nossos testes, utilizamos $5 \times$ epsopt como a precisão pedida para resolver o primeiro subproblema e denotamos essa implementação por aprox5. Os perfis de desempenhos de aprox5 e de exato são exibidos no Gráfico 5.6, pelo qual podemos ver que empregar uma precisão menor na primeira execução do Gencan aumenta a velocidade do Algoritmo (APB) e reduz muito pouco sua robustez, pois aprox5 resolve um problema a menos que exato.

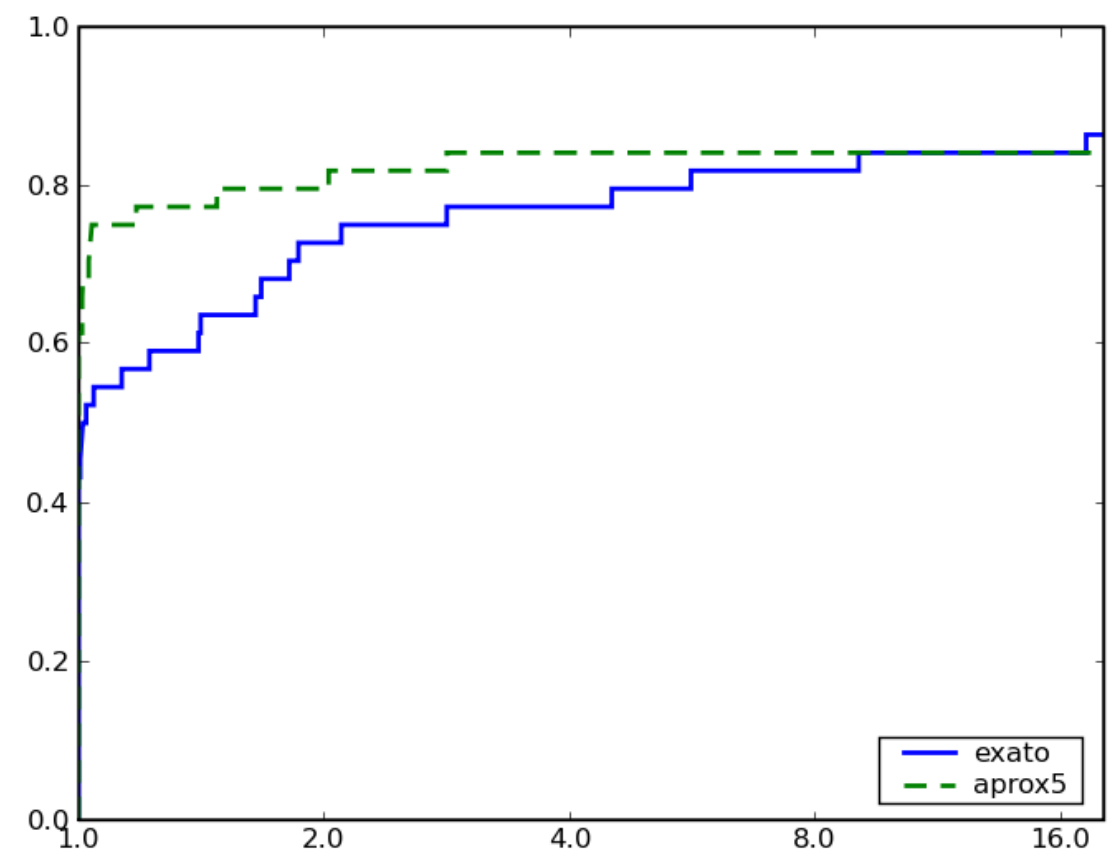

Figura 5.6: Comparação entre a implementação exata e a que adota uma precisão menor para resolver o primeiro subproblema irrestrito.

Contudo, nossa escolha da precisão pedida para resolver o primeiro subproblema irrestrito foi empírica e não obedece um critério baseado na inviabilidade do ponto atual, como os descritos em [Ber82, Ber99]. De fato, se escolhermos um par de pontos iniciais $\left(x^{0}, U_{0}\right)$ muito próximos de um par de soluções dos problemas primal e dual, devemos pedir a pre- 
cisão epsopt na primeira execução do GEnCAN. Por outro lado, se o par $\left(x^{0}, U_{0}\right)$ estiver muito longe de um par de soluções primal e dual, tal precisão pode ser mais relaxada ainda que a empregada em aprox5. Tais observações nos motivaram a estudar a próxima estratégia.

Na segunda alternativa, baseada em [Ber99, pág. 415], escolhemos duas seqüências monótonas não-crescentes $\left\{\epsilon_{k}\right\}$ e $\left\{\beta_{k}\right\}$ que convergem a 0 e definimos $\rho: \mathbb{R}^{n} \rightarrow \mathbb{R}_{+}$por

$$
\rho(x) \stackrel{\text { def }}{=} \mid \lambda_{\max }\left(A_{0}+\mathcal{A}(x)\right)-\text { epsfeas }\left.\right|_{+}=\max \left\{0, \lambda_{\max }\left(A_{0}+\mathcal{A}(x)\right)-\text { epsfeas }\right\}
$$

para medir a viabilidade de $x \in \mathbb{R}^{n}$ em relação à restrição matricial do problema primal. A cada iteração $k$ do algoritmo de lagrangianos aumentados, é procurada uma solução aproximada $x^{*}$ do subproblema irrestrito que satisfaça

$$
\left\|\nabla_{x} \mathrm{~L}_{p_{k}}^{+}\left(x^{*}, V_{k}\right)\right\| \leq \max \left\{\text { epsopt, } \min \left\{\epsilon_{k}, \beta_{k} \rho\left(x^{*}\right)\right\}\right\} .
$$

Tal critério impõe uma precisão maior para pontos viáveis do problema primal e relaxa tal precisão para pontos inviáveis, já que não podem ser soluções primais. Em nossos testes, escolhemos $\epsilon_{0}=10^{-2} \mathrm{e}$

$$
\beta_{0}= \begin{cases}1.0 & \text { se } \rho_{0}\left(x^{0}\right)=0.0 \\ \max \left\{\frac{1.0}{\rho_{0}\left(x_{0}\right)}, 1.0\right\} & \text { caso contrário }\end{cases}
$$

Atualizamos esses parâmetros a cada iteração $k$ do Algoritmo (APB) através das fórmulas:

$$
\begin{aligned}
\beta_{k} & =\beta_{k-1} * \delta_{\beta}, \\
\epsilon_{k} & =\epsilon_{k-1} * \delta_{\epsilon},
\end{aligned}
$$

em que $\delta_{\beta}=\delta_{\epsilon}=0.5$. Denotamos essa implementação por aprox-berts e comparamos seu desempenho com o da versão exata no Gráfico 5.7. A partir desses perfis de desempenho, observamos que a estratégia aprox-berts é uma das mais eficientes em quase $90 \%$ dos problemas de teste e também é mais robusta que a implementação exata, resolvendo mais de $93 \%$ dos problemas de teste (41 dos 44 problemas).

Visto que a implementação aprox5 também é mais eficiente que a versão exato, embora possua quase a mesma robustez, decidimos compará-la com aprox-berts para verificarmos qual delas é mais eficiente. Pelo Gráfico 5.8, observamos que as duas implementações possuem eficiências similares, o que não nos permite concluir claramente qual das duas é a mais rápida.

Portanto, a obtenção de soluções aproximadas para os subproblemas irrestritos do algoritmo de lagrangianos aumentados resulta em melhorias na velocidade do método. Particularmente, a adoção do critério de Bertsekas melhora consideravelmente a eficiência e a robustez do algoritmo.

\subsection{Conclusões sobre os experimentos}

Neste capítulo, abordamos algumas implementações do algoritmo de lagrangianos aumentados estudado no Capítulo 4. Tais implementações foram feitas a partir da adaptação 


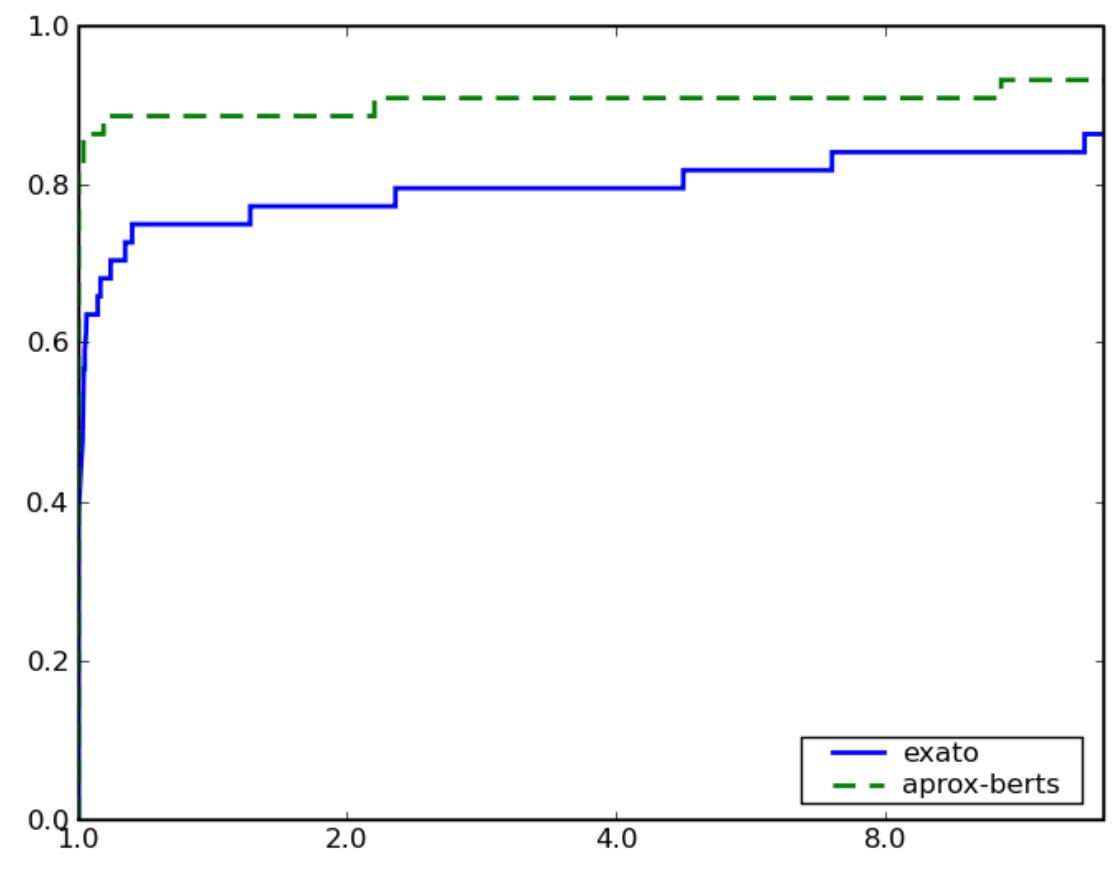

Figura 5.7: Comparação entre a implementação exata e a que adota o critério de Bertsekas para resolver os subproblemas do algoritmo de lagrangianos aumentados.

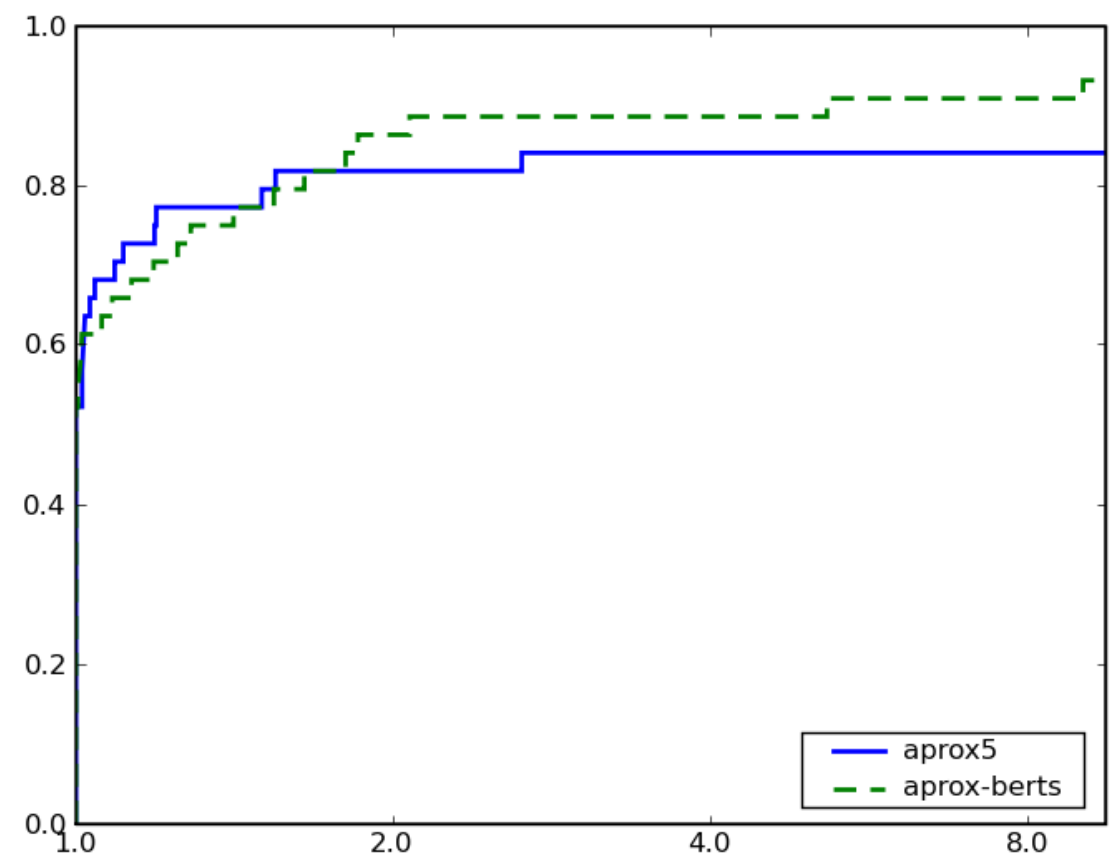

Figura 5.8: Comparação entre as implementações que resolvem de maneira aproximada os subproblemas do Algoritmo (APB). 
das estruturas dos programas Algencan e GENCAn e tiveram como objetivo comparar diferentes escolhas dos parâmetros, as possíveis vantagens pelo uso da estrutura de blocos diagonais das matrizes e os critérios para a resolução dos subproblemas irrestritos. A partir da análise dos resultados dos experimentos, escolhemos a estratégia aprox-berts, devido ao seu melhor desempenho, tanto em robustez quanto em eficiência. Tal implementação tem como características: a escolha de um múltiplo elevado da matriz identidade como ponto inicial do problema dual, o aproveitamento da estrutura em blocos diagonais das matrizes do problema, a utilização da hessiana real do lagrangiano aumentado pelo método de gradiente conjugados do GENCAN e um critério baseado em [Ber82, Ber99] para resolver os subproblemas irrestritos a cada iteração do algoritmo.

Apresentamos na Tabela 5.5 algumas informações da estratégia escolhida, tais como:

- tempo: tempo gasto, em segundos, para resolver cada problema. Vale ressaltar que a medida de tempo apresentada na tabela não considera o tempo necessário para ler os dados do problema a partir de arquivos.

- inform: código de retorno do algoritmo. O código 0 indica que o problema foi resolvido, -1 indica que o problema não foi resolvido dentro do limite de duas horas de uso do processador e 4 representa que o programa foi encerrado devido à falta de progresso (redução de inviabilidade ou complementaridade);

- n, m: dimensões do problema;

- outiter: número de iterações do algoritmo de lagrangianos aumentados;

- totiter: número total de iterações do GENCAN para resolver os subproblemas irrestritos;

- totfcnt: número de avaliações do lagrangiano aumentado;

- totgcnt: número de avaliações do gradiente do lagrangiano aumentado;

- totcgcnt: número de total de iterações do método de gradientes conjugados do GenCAN;

- $f$ : valor da função objetivo na última iteração do algoritmo de lagrangianos aumentados.

Além disso, observamos que usar explicitamente o padrão de esparsidade das matrizes do problema pode trazer ganhos substanciais para o desempenho do algoritmo. Particularmente, pela discussão feita na Seção 5.2 , se a seqüência de matrizes $\left\{U_{k}\right\}$ gerada pelo algoritmo possuísse a mesma estrutura em blocos diagonais que as matrizes $A_{0}, \ldots, A_{n}$ do problema, então seria reduzido o custo de avaliar a hessiana do lagrangiano aumentado, a qual é uma das operações mais custosas do método. Todavia, tal propriedade não é garantida pelo Algoritmo (APB) quando é definida a matriz $U_{k}$ pelas equações (4.8) e (4.9), para $k \geq 1$. Por tal motivo, consideramos interessante estudar algoritmos que consigam aproveitar tais padrões de esparsidade. Um programa que possui essas características e que tem apresentado bons resultados práticos é o PENNON [KS01, KS03], desenvolvido por Kočvara e Stingl e baseado no algoritmo de lagrangianos aumentados de Ben-Tal e Zibulevsky [BTZ97], da mesma maneira que o Algoritmo (APB). 


\begin{tabular}{|c|c|c|c|c|c|c|c|c|c|c|}
\hline problema & tempo(s) & inform & $\mathbf{n}$ & $\mathbf{m}$ & outiter & totiter & totfcnt & totgcnt & totcgent & $\mathrm{f}$ \\
\hline control1 & 0.28 & 0 & 21 & 15 & 41 & 208 & 324 & 295 & 4568 & $1.77846850 \mathrm{e}+01$ \\
\hline control2 & 5.31 & 0 & 66 & 30 & 39 & 311 & 476 & 393 & 25638 & $8.30001889 e+00$ \\
\hline control3 & 65.27 & 0 & 136 & 45 & 53 & 501 & 832 & 612 & 84475 & $1.36332807 \mathrm{e}+01$ \\
\hline control4 & 495.89 & 0 & 231 & 60 & 41 & 800 & 1422 & 885 & 240858 & $1.97943929 \mathrm{e}+01$ \\
\hline gpp100 & 1309.93 & 0 & 101 & 100 & 49 & 1508 & 19400 & 1609 & 59519 & $-4.49435508 e+01$ \\
\hline gpp124-1 & 740.46 & 0 & 125 & 124 & 47 & 351 & 8223 & 448 & 22917 & $-7.34307634 \mathrm{e}+00$ \\
\hline gpp124-2 & 5040.49 & 0 & 125 & 124 & 49 & 2209 & 65687 & 2316 & 32415 & $-4.68622957 \mathrm{e}+01$ \\
\hline gpp124-3 & 240.01 & 0 & 125 & 124 & 50 & 135 & 1503 & 237 & 4866 & $-1.53014127 \mathrm{e}+02$ \\
\hline gpp124-4 & 3396.48 & 0 & 125 & 124 & 51 & 1270 & 57976 & 1374 & 12695 & $-4.18987626 e+02$ \\
\hline gpp250-1 & 0.00 & -1 & 251 & 250 & 19 & 156 & 2660 & 197 & 18061 & $-1.55710190 \mathrm{e}+01$ \\
\hline gpp 250-2 & 3078.69 & 0 & 251 & 250 & 53 & 130 & 642 & 239 & 6257 & $-8.18689588 e+01$ \\
\hline gpp 250-3 & 2541.43 & 0 & 251 & 250 & 54 & 108 & 410 & 218 & 5275 & $-3.03539324 \mathrm{e}+02$ \\
\hline gpp250-4 & 0.00 & -1 & 251 & 250 & 52 & 127 & 290 & 233 & 7482 & $-7.47328311 \mathrm{e}+02$ \\
\hline hinf1 & 37.49 & 0 & 13 & 14 & 41 & 10140 & 353555 & 10228 & 46445 & $2.03258885 \mathrm{e}+00$ \\
\hline hinf2 & 0.60 & 0 & 13 & 16 & 40 & 778 & 1945 & 862 & 18330 & $1.09670605 \mathrm{e}+01$ \\
\hline hinf3 & 0.49 & 0 & 13 & 16 & 32 & 340 & 2252 & 405 & 6597 & $5.69425780 \mathrm{e}+01$ \\
\hline hinf4 & 0.25 & 0 & 13 & 16 & 39 & 235 & 882 & 326 & 4103 & $2.74763848 \mathrm{e}+02$ \\
\hline hinf5 & 0.65 & 0 & 13 & 16 & 37 & 723 & 2334 & 803 & 16060 & $3.62357016 \mathrm{e}+02$ \\
\hline hinf6 & 1.70 & 0 & 13 & 16 & 47 & 1881 & 7147 & 1980 & 42792 & $4.48930209 \mathrm{e}+02$ \\
\hline hinf7 & 5.81 & 0 & 13 & 16 & 41 & 5559 & 29702 & 5648 & 119969 & $3.90813295 \mathrm{e}+02$ \\
\hline hinf8 & 1.50 & 0 & 13 & 16 & 36 & 1674 & 5947 & 1755 & 37285 & $1.16147554 \mathrm{e}+02$ \\
\hline hinf9 & 2.35 & 0 & 13 & 16 & 34 & 3165 & 8655 & 3241 & 66048 & $2.36249515 \mathrm{e}+02$ \\
\hline hinf10 & 1.02 & 0 & 21 & 18 & 43 & 608 & 3238 & 775 & 9266 & $1.08712426 \mathrm{e}+02$ \\
\hline hinf11 & 0.88 & 0 & 31 & 22 & 44 & 393 & 516 & 485 & 10859 & $6.59178766 \mathrm{e}+01$ \\
\hline hinf12 & 2.67 & 0 & 43 & 24 & 46 & 546 & 806 & 646 & 28439 & $2.19402690 \mathrm{e}-02$ \\
\hline hinf13 & 81.02 & 0 & 57 & 30 & 46 & 4534 & 71287 & 4667 & 165540 & $4.43669241 \mathrm{e}+01$ \\
\hline hinf14 & 83.69 & 0 & 73 & 34 & 42 & 5166 & 5280 & 5256 & 743440 & $1.29907378 \mathrm{e}+01$ \\
\hline hinf15 & 2689.60 & 4 & 91 & 37 & 18 & 66141 & 71513 & 66182 & 11280996 & $7.22835664 \mathrm{e}+00$ \\
\hline $\operatorname{mcp} 100$ & 71.17 & 0 & 100 & 100 & 49 & 91 & 204 & 193 & 2374 & $2.26157351 \mathrm{e}+02$ \\
\hline mcp124-1 & 161.82 & 0 & 124 & 124 & 49 & 103 & 202 & 202 & 3623 & $1.41990477 \mathrm{e}+02$ \\
\hline $\operatorname{mcp} 124-2$ & 153.79 & 0 & 124 & 124 & 50 & 97 & 198 & 198 & 3043 & $2.69880170 \mathrm{e}+02$ \\
\hline mcp124-3 & 166.24 & 0 & 124 & 124 & 50 & 105 & 210 & 207 & 4294 & $4.67750114 \mathrm{e}+02$ \\
\hline mcp $124-4$ & 148.43 & 0 & 124 & 124 & 51 & 93 & 205 & 198 & 2528 & $8.64411864 \mathrm{e}+02$ \\
\hline mcp 250-1 & 2688.88 & 0 & 250 & 250 & 52 & 118 & 231 & 224 & 6885 & $3.17264340 \mathrm{e}+02$ \\
\hline mсp 250-2 & 2220.14 & 0 & 250 & 250 & 53 & 98 & 205 & 205 & 4398 & $5.31930084 \mathrm{e}+02$ \\
\hline mcp $250-3$ & 2291.36 & 0 & 250 & 250 & 54 & 101 & 210 & 210 & 4570 & $9.81172572 \mathrm{e}+02$ \\
\hline $\operatorname{mcp} 250-4$ & 2206.22 & 0 & 250 & 250 & 54 & 96 & 217 & 207 & 4780 & $1.68196011 \mathrm{e}+03$ \\
\hline qap5 & 318.34 & 0 & 136 & 26 & 45 & 5054 & 5326 & 5150 & 1359403 & $-4.35999997 \mathrm{e}+02$ \\
\hline qap6 & 1737.52 & 0 & 229 & 37 & 41 & 5202 & 5773 & 5303 & 2180597 & $-3.81422697 \mathrm{e}+02$ \\
\hline thetal & 13.58 & 0 & 104 & 50 & 41 & 79 & 175 & 165 & 3607 & $2.30000000 \mathrm{e}+01$ \\
\hline truss1 & 0.04 & 0 & 6 & 13 & 41 & 76 & 170 & 160 & 360 & $-8.99999632 \mathrm{e}+00$ \\
\hline truss2 & 40.24 & 0 & 58 & 133 & 51 & 218 & 331 & 322 & 9489 & $-1.23380358 \mathrm{e}+02$ \\
\hline truss 3 & 0.62 & 0 & 27 & 31 & 40 & 119 & 204 & 200 & 3056 & $-9.10999657 \mathrm{e}+00$ \\
\hline truss 4 & 0.12 & 0 & 12 & 19 & 41 & 94 & 181 & 178 & 930 & $-9.00999629 \mathrm{e}+00$ \\
\hline
\end{tabular}

Tabela 5.7: Informações da implementação com melhor desempenho nos testes númericos.

Além disso, outro ponto que contribuiu significativamente para a melhoria da robustez e da eficiência do algoritmo implementado foi a resolução aproximada dos subproblemas irrestritos a cada iteração. Logo, também é importante analisarmos outros critérios para aceitar uma solução aproximada dos subproblemas. Um critério de nosso interesse é o apresentado por Eckstein [Eck03], o qual possui boas propriedades de convergência da seqüência gerada de soluções aproximadas para um par de soluções do problemas primal e dual. 


\section{Capítulo 6}

\section{Conclusão}

Neste trabalho, estudamos métodos de multiplicadores para a resolução de problemas de Programação Semidefinida Convexa (PSD). A abordagem dos trabalhos analisados baseouse na extensão de algoritmos conhecidos de lagrangianos aumentados para a resolução dessa classe de problemas.

Inicialmente, estudamos as características da subclasse de problemas de Programação Semidefinida de nosso interesse, denotada (PSDA), com ênfase na análise do problema dual e nas condições para a existência de soluções para os problemas primal e dual.

Com base nesses conceitos, nós analisamos um método de multiplicadores exponenciais proposto por Doljansky e Teboulle [DT99] para resolver (PSDA) e o método de ponto proximal interior associado a ele para resolver o problema dual. Tal método de ponto proximal usa uma regularização baseada na entropia de Kullback-Leibler e estende resultados conhecidos para programação não-linear no $\mathbb{R}^{n}$.

Em seguida, estudamos o algoritmo de lagrangianos aumentados suaves introduzido por Mosheyev e Zibulevsky em [MZ00] e que aplica as idéias de algoritmos de penaliddade e barreira de Ben-Tal e Zibulevsky [BTZ97] para resolver problemas da classe (PSDA). Tal abordagem possibilita o uso de diferentes lagrangianos aumentados a partir da escolha de uma função penalidade que atenda certas condições. A análise de convergência feita segue idéias similares àquelas abordadas por Doljansky e Teboulle. Contudo, o papel diferente exercido pelo multiplicador em relação aos métodos de multiplicadores usuais ajudou a explicar o fato dos autores não terem obtido melhores resultados de convergência das seqüências geradas pelo método.

Por fim, fizemos testes numéricos para avaliar o desempenho do algoritmo proposto por Mosheyev e Zibulevsky. Analisamos o efeito da escolha de alguns parâmetros, do aproveitamento da esparsidade das matrizes do problema e da resolução aproximada dos subproblemas irrestritos em cada iteração do algoritmo de lagrangianos aumentados. A partir da análise dos testes, escolhemos a implementação que adota um ponto inicial dual distante da borda do cone de matrizes semidefinidas positivas, aproveita a estrutura de blocos diagonais das matrizes dos problemas e emprega um critério proposto por Bertsekas [Ber82, Ber99] para aceitar uma solução aproximada dos subproblemas.

A partir deste trabalho, surgiram novas questões para pesquisas futuras, principalmente:

- Estudo de outros métodos de multiplicadores para resolver problemas de programação convexa semidefinida e que aproveitem os padrões de esparsidade das matrizes dos 
problemas, como o algoritmo PENNON [KS01, KS03], introduzido por Kočvara e Stingl.

- Análise de outros critérios para a aceitação dos subproblemas irrestritos dos métodos de multiplicadores. Particularmente, um de nossos principais interesses está naquele apresentado por Eckstein [Eck03]. 


\section{Apêndice A}

\section{Seqüências}

Neste apêndice, apresentamos alguns resultados sobre a convergência de seqüências de vetores do $\mathbb{R}^{n}$ que serão importantes para analisar os algoritmos abordados no Capítulo 3 .

Consideremos a seqüência obtida pela avaliação da função entropia de Kullback-Leibler no par de elementos de duas seqüências limitadas. O próximo resultado mostra que se essa seqüência converge a zero, então a diferença das duas seqüências limitadas converge a zero também.

Lema A.1 ([DT99, Lema A.1]). Sejam $\left\{a_{k}\right\},\left\{b_{k}\right\} \subseteq \mathbb{R}_{++}$seqüências limitadas e $\mathrm{d}_{\mathrm{KL}}: \mathbb{R}_{+} \times$ $\mathbb{R}_{++}$a função entropia relativa de Kullback-Leibler definida por $\mathrm{d}_{\mathrm{KL}}(s, t) \stackrel{\text { def }}{=} s \log s-s \log t+$ $t-s$. Se $\mathrm{d}_{\mathrm{KL}}\left(a_{k}, b_{k}\right) \rightarrow 0$ quando $k \rightarrow \infty$, então $\left(a_{k}-b_{k}\right) \rightarrow 0$ também.

Demonstração. Seja $\left\{c_{k}\right\}$ uma seqüência definida por $c_{k} \stackrel{\text { def }}{=} a_{k}-b_{k}$. Já que $\left\{a_{k}\right\}$ e $\left\{b_{k}\right\}$ são limitadas, então $\left\{c_{k}\right\}$ também é limitada. Provemos que $c_{k} \rightarrow 0$.

Suponhamos que a seqüência $\left\{c_{k}\right\}$ possua um ponto limite $\bar{c} \neq 0$. Sem perda de generalidade, consideremos que $c_{k} \rightarrow \bar{c}$. Seja $\left\{a_{k_{j}}\right\}$ uma subseqüência de $\left\{a_{k}\right\}$ que convirja para $\bar{a} \geq 0$ e $\left\{b_{k_{j_{l}}}\right\}$ uma seqüência de $\left\{b_{k_{j}}\right\}$ convergindo para $\bar{b} \geq 0$. Seja $r \stackrel{\text { def }}{=} k_{j_{l}}$. Assim, $a_{r}-b_{r} \rightarrow \bar{a}-\bar{b}$ e $c_{r} \rightarrow \bar{c}$ quando $r \rightarrow \infty$. Já que $c_{r}=a_{r}-b_{r}$, temos $\bar{c}=\bar{a}-\bar{b}$. Analisemos todas as possibilidades para os valores de $\bar{a}$ e $\bar{b}$ :

(i) $\bar{a}=\bar{b}=0$. Obtemos $0 \neq \bar{c}=\bar{a}-\bar{b}=0$, uma contradição;

(ii) $\bar{a}>0$ e $\bar{b}>0$. Já que $\mathrm{d}_{\mathrm{KL}}$ é contínua em $\mathbb{R}_{+} \times \mathbb{R}_{++}$, segue que $\mathrm{d}_{\mathrm{KL}}\left(a_{r}, b_{r}\right) \rightarrow \mathrm{d}_{\mathrm{KL}}(\bar{a}, \bar{b})$. Por hipótese, nós sabemos que $\mathrm{d}_{\mathrm{KL}}\left(a_{r}, b_{r}\right) \rightarrow 0$, resultando que $\mathrm{d}_{\mathrm{KL}}(\bar{a}, \bar{b})=0$. Já que $\mathrm{d}_{\mathrm{KL}}(s, t)=0$ se e somente se $s=t$, obtemos $\bar{c}=\bar{a}-\bar{b}=0$, uma contradição.

(iii) $\bar{a}=0$ e $\bar{b}>0$. Usando o mesmo argumento do item (ii), obtemos uma contradição.

(iv) $\bar{a}>0$ e $\bar{b}=0$. Pela definição de $\mathrm{d}_{\mathrm{KL}}$ e pelo fato de $\left\{a_{r}\right\}$ ser limitada, podemos ver que $\mathrm{d}_{\mathrm{KL}}\left(a_{r}, b_{r}\right) \rightarrow \infty$, o que contradiz a hipótese de $\mathrm{d}_{\mathrm{KL}}\left(a_{r}, b_{r}\right) \rightarrow 0$.

Portanto, $\bar{c}=0$. Devido ao fato de $\left\{c_{k}\right\}$ ser uma seqüência limitada, segue que $c_{k} \rightarrow 0$, o que encerra a demonstração. 
O próximo teorema mostra que a seqüência ergódica associada a uma seqüência convergente também converge sob algumas condições.

Teorema A.2. Sejam $\left\{x^{k}\right\} \subset \mathbb{R}^{n}$ uma seqüência convergente tal que $\lim _{k \rightarrow \infty} x^{k}=\bar{x}$ e $\left\{\alpha_{k}\right\} \subset \mathbb{R}_{+}$uma seqüência de escalares não-negativos tais que $\sum_{i=1}^{\infty} \alpha_{i}=\infty$. Então, a seqüência ergódica dada por

$$
\hat{x}^{k}=\sum_{i=1}^{k} \frac{\alpha_{i}}{\sum_{j=1}^{k} \alpha_{j}} x^{i}=\frac{\sum_{i=1}^{k} \alpha_{i} x^{i}}{\sum_{i=1}^{k} \alpha_{i}}
$$

converge para $\bar{x}$ também, ou seja, $\lim _{k \rightarrow \infty} \hat{x}^{k}=\bar{x}$.

Demonstração. Para todo $\epsilon>0$, sabemos que existe um inteiro estritamente positivo $k_{\epsilon}$ tal que

$$
\left\|x^{k}-\bar{x}\right\|<\epsilon, \quad \text { para todo } k \geq k_{\epsilon} .
$$

Consideremos a seqüência $\left\{\hat{x}^{k}\right\}$. Para todo $\epsilon>0$ e $k \geq k_{\epsilon}$, temos

$$
\begin{aligned}
\left\|\hat{x}^{k}-\bar{x}\right\| & =\left\|\frac{\sum_{i=1}^{k} \alpha_{i} x^{i}}{\sum_{i=1}^{k} \alpha_{i}}-\bar{x}\right\|=\left\|\frac{\sum_{i=1}^{k} \alpha_{i} x^{i}}{\sum_{i=1}^{k} \alpha_{i}}-\frac{\sum_{i=1}^{k} \alpha_{i} \bar{x}}{\sum_{i=1}^{k} \alpha_{i}}\right\| \\
& =\left\|\frac{\sum_{i=1}^{k_{\epsilon}-1} \alpha_{i}\left(x^{i}-\bar{x}\right)}{\sum_{i=1}^{k} \alpha_{i}}+\frac{\sum_{i=k_{\epsilon}}^{k} \alpha_{i}\left(x^{i}-\bar{x}\right)}{\sum_{i=1}^{k} \alpha_{i}}\right\| \\
& \leq\left\|\frac{\sum_{i=1}^{k_{\epsilon}-1} \alpha_{i}\left(x^{i}-\bar{x}\right)}{\sum_{i=1}^{k} \alpha_{i}}\right\|+\left\|\frac{\sum_{i=k_{\epsilon}}^{k} \alpha_{i}\left(x^{i}-\bar{x}\right)}{\sum_{i=1}^{k} \alpha_{i}}\right\| \\
& =\frac{\left\|\sum_{i=1}^{k_{\epsilon}-1} \alpha_{i}\left(x^{i}-\bar{x}\right)\right\|}{\sum_{i=1}^{k} \alpha_{i}}+\frac{\left\|\sum_{i=k_{\epsilon}}^{k} \alpha_{i}\left(x^{i}-\bar{x}\right)\right\|}{\sum_{i=1}^{k_{\epsilon}-1} \alpha_{i}+\sum_{i=k_{\epsilon}}^{k} \alpha_{i}} \\
& \leq \frac{\sum_{i=1}^{k_{\epsilon}-1} \alpha_{i}\left\|x^{i}-\bar{x}\right\|}{\sum_{i=1}^{k} \alpha_{i}}+\sum_{i=k_{\epsilon}}^{k} \frac{\alpha_{i}\left\|x^{i}-\bar{x}\right\|}{\sum_{i=k_{\epsilon}}^{k} \alpha_{i}} \\
& \leq \frac{\sum_{i=1}^{k_{\epsilon}-1} \alpha_{i}\left\|x^{i}-\bar{x}\right\|}{\sum_{i=1}^{k} \alpha_{i}}+\sum_{i=k_{\epsilon}}^{k} \frac{\alpha_{i} \epsilon}{\sum_{i=k_{\epsilon}}^{k} \alpha_{i}} \\
& =\frac{\sum_{i=1}^{k_{\epsilon}-1} \alpha_{i}\left\|x^{i}-\bar{x}\right\|}{\sum_{i=1}^{k} \alpha_{i}}+\epsilon
\end{aligned}
$$

Logo,

$$
0 \leq\left\|\hat{x}^{k}-\bar{x}\right\| \leq \frac{\sum_{i=1}^{k_{\epsilon}-1} \alpha_{i}\left\|x^{i}-\bar{x}\right\|}{\sum_{i=1}^{k} \alpha_{i}}+\epsilon,
$$

para todo $\epsilon>0$ e $k \geq k_{\epsilon}$. Já que $\sum_{i=1}^{k} \alpha_{i} \rightarrow \infty$, segue que

$$
\lim _{k \rightarrow \infty} \frac{\sum_{i=1}^{k_{\epsilon}-1} \alpha_{i}\left\|x^{i}-\bar{x}\right\|}{\sum_{i=1}^{k} \alpha_{i}}=0,
$$


para todo $\epsilon>0$. Então, para todo $\epsilon>0$, ao tomarmos o limite para $k \rightarrow \infty$ na expressão (A.2), obtemos

$$
0 \leq \lim _{k \rightarrow \infty}\left\|\hat{x}^{k}-\bar{x}\right\| \leq \epsilon
$$

resultando que $\lim _{k \rightarrow \infty}\left\|\hat{x}^{k}-\bar{x}\right\|=0$, o que equivale a $\lim _{k \rightarrow \infty} \hat{x}^{k}=\bar{x}$. 


\section{Apêndice B}

\section{Teorema generalizado de Gordan}

O próximo teorema, um caso particular de separação de dois conjuntos convexos, pode ser visto como uma extensão do Teorema generalizado de Gordan [Man69, Teorema 4.2.3] para funções $\succeq$-convexas. Esse resultado é usado no Capítulo 2 na demonstração de um teorema que apresenta condições que garantem que a folga de dualidade é zero e que o problema dual tem solução.

Teorema B.1 (Baseado em [Roc70, Teorema 21.1] e [Man69, Teorema 4.2.3]). Sejam $C \subseteq \mathbb{R}^{n}$ um conjunto convexo, $f_{i}: \mathbb{R}^{n} \rightarrow(-\infty, \infty], i=1, \ldots, r$, funções convexas próprias $e G_{j}: \mathbb{R}^{n} \rightarrow \mathbb{S}^{m}, j=1, \ldots, s$, funções $\succeq$-convexas. Suponha que $\operatorname{dom} f_{i} \supseteq \operatorname{ri} C, i=1, \ldots, r$. Então, exatamente uma das seguintes afirmações é válida:

(a) Existe $\hat{x} \in C$ tal que

$$
\begin{aligned}
f_{i}(\hat{x})<0, & i=1, \ldots, r \\
G_{j}(\hat{x}) \prec 0, & j=1, \ldots, s .
\end{aligned}
$$

(b) Existem $\lambda_{1}, \ldots, \lambda_{r} \in \mathbb{R}_{+}$e $U_{1}, U_{2}, \ldots, U_{s} \in \mathbb{S}_{+}^{m}$ tais que, para todo $x \in C$,

$$
\lambda_{1} f_{1}(x)+\cdots+\lambda_{r} f_{r}(x)+\left\langle G_{1}(x), U_{1}\right\rangle+\cdots+\left\langle G_{s}(x), U_{s}\right\rangle \geq 0
$$

$e$, além disso, $\lambda_{i}>0$ para algum $i \in\{1, \ldots, s\}$ ou $U_{j} \neq 0$ para algum $j \in\{1, \ldots, s\}$.

Demonstração. Consideremos que a afirmação $(a)$ seja válida. Seja $\hat{x}$ um ponto que satisfaça (B.1). Logo, para quaisquer $\lambda_{1}, \ldots, \lambda_{r} \in \mathbb{R}_{+}$e $U_{1}, \ldots, U_{s} \in \mathbb{S}_{+}^{m}$, sabemos pelo Corolário 1.3.7 que

$$
\lambda_{1} f_{1}(\hat{x})+\cdots+\lambda_{r} f_{r}(\hat{x})+\left\langle G_{1}(\hat{x}), U_{1}\right\rangle+\cdots+\left\langle G_{s}(\hat{x}), U_{s}\right\rangle \leq 0 .
$$

Além disso, se $\lambda_{i}>0$ para algum $i \in\{1, \ldots, r\}$ ou $U_{j} \neq 0$ para algum $j \in\{1, \ldots, s\}$, então, pela Proposição 1.3.8, a desigualdade é estrita na inequação acima. Portanto, a afirmação (b) não vale.

Suponhamos agora que $(a)$ seja falsa. Se $C$ é vazio, então a afirmação $(b)$ trivialmente é válida. Consideremos então que $C$ seja não-vazio. Logo, ri $C$ é não-vazio também.

Denotemos por $C_{1}$ o conjunto das tuplas $\left(\mu_{1}, \ldots, \mu_{r}, H_{1}, \ldots, H_{s}\right)$ tais que $\mu_{1}, \ldots, \mu_{r} \in$ $\mathbb{R}, H_{1}, \ldots, H_{s} \in \mathbb{S}^{m}$ e existe $x \in C \operatorname{com} f_{i}(x)<\mu_{i}$ e $G_{j}(x) \prec H_{j}$, para $i=1, \ldots, r$ e 
$j=1, \ldots, s$. Tal conjunto é não-vazio. De fato, seja $x^{\circ} \in$ ri $C$. Por hipótese, sabemos que $f_{i}\left(x^{\circ}\right)<\infty$, para $i=1, \ldots, r$. Então, para qualquer real positivo $\epsilon$, o ponto $\left(f_{1}\left(x^{\circ}\right)+\right.$ $\left.\epsilon, \ldots, f_{r}\left(x^{\circ}\right)+\epsilon, G_{1}\left(x^{\circ}\right)+\epsilon I, \ldots, G_{s}\left(x^{\circ}\right)+\epsilon I\right)$ pertence a $C_{1}$.

Mostremos que $C_{1}$ é convexo. Sejam $\alpha \in[0,1], v \stackrel{\text { def }}{=}\left(\sigma_{1}, \ldots, \sigma_{r}, Y_{1}, \ldots, Y_{s}\right)$ e $w \stackrel{\text { def }}{=}$ $\left(\delta_{1}, \ldots, \delta_{r}, Z_{1}, \ldots, Z_{s}\right)$ elementos de $C_{1}$. Sabemos que existem $y, z \in C$ tais que $f_{i}(y)<\sigma$, $G_{j}(y) \prec Y_{j}, f_{i}(z)<\delta$ e $G_{j}(z) \prec Z_{j}$ para $i=1, \ldots, r$ e $j=1, \ldots, s$. Pela hipótese de $C$ ser convexo, obtemos $\alpha y+(1-\alpha) z \in C$. E devido a $f_{i}$ ser convexa para $i=1, \ldots, r$, podemos ver que

$$
\begin{aligned}
f_{i}(\alpha y+(1-\alpha) z) & \leq \alpha f_{i}(y)+(1-\alpha) f(z), \quad i=1, \ldots, r \\
& <\alpha \sigma_{i}+(1-\alpha) \delta_{i}, \quad i=1, \ldots, r .
\end{aligned}
$$

Pela hipótese de $G_{j}$ ser $\succeq$-convexa para $j=1, \ldots, s$, temos

$$
\begin{aligned}
G_{j}(\alpha y+(1-\alpha) z) & \preceq \alpha G_{j}(y)+(1-\alpha) G_{j}(z), \quad j=1, \ldots, s, \\
& \prec \alpha Y_{j}+(1-\alpha) Z_{j}, \quad j=1, \ldots, s .
\end{aligned}
$$

E por (B.3) e (B.4), concluímos que $\alpha v+(1-\alpha) w \in C_{1}$. Portanto, $C_{1}$ é convexo.

Seja $C_{2}$ o conjunto composto por pontos $\left(\mu_{1}, \ldots, \mu_{r}, H_{1}, \ldots, H_{s}\right)$ tais que $\mu_{1}, \ldots, \mu_{r} \leq 0$ e $H_{1}, \ldots, H_{s} \preceq 0$. Já que (a) é falsa, é fácil ver que $C_{2}$ é disjunto de $C_{1}$. Além disso, o conjunto $C_{2}$ é convexo e não-vazio, pois é o produto cartesiano de conjuntos convexos e contém o ponto $(0, \ldots, 0,0, \ldots, 0)$, respectivamente. Logo, por uma extensão dos resultados apresentados em [Roc70, Teorema 11.3] e [Ber03, Proposição 2.4.6], os conjuntos $C_{1}$ e $C_{2}$ podem ser separados por um hiperplano, o que equivale a existir $\lambda=\left(\lambda_{1}, \ldots, \lambda_{r}\right) \in \mathbb{R}^{r}$, $U_{1}, \ldots, U_{s} \in \mathbb{S}^{m}, \eta \in \mathbb{R}$ tais que

$$
\begin{aligned}
& \eta \leq \sum_{i=1}^{r} \lambda_{i} \nu_{i}+\left\langle U_{1}, V_{1}\right\rangle+\cdots+\left\langle U_{s}, V_{s}\right\rangle, \quad \forall\left(\nu_{1}, \ldots, \nu_{r}, V_{1}, \ldots, V_{s}\right) \in C_{1}, \\
& \eta \geq \sum_{i=1}^{r} \lambda_{i} \omega_{i}+\left\langle U_{1}, W_{1}\right\rangle+\cdots+\left\langle U_{s}, W_{s}\right\rangle, \quad \forall\left(\omega_{1}, \ldots, \omega_{r}, W_{1}, \ldots, W_{s}\right) \in C_{2} .
\end{aligned}
$$

e, além disso, $\lambda_{i} \neq 0$, para algum $i \in\{1, \ldots, r\}$, ou $U_{j} \neq 0$, para algum $j \in\{1, \ldots, s\}$.

Suponhamos que $\lambda_{k}<0$ para algum $k \in\{1, \ldots, r\}$ ou que $U_{l} \nsucceq 0$ para algum $l \in$ $\{1, \ldots, s\}$. A partir do Teorema de Fejer (Proposição 1.3.6) e pela escolha do conjunto $C_{2}$, podemos ver que a desigualdade (B.5b) é violada por um ponto $\left(\tilde{\omega}_{1}, \ldots, \tilde{\omega}_{r}, \tilde{W}_{1}, \ldots, \tilde{W}_{s}\right) \in$ $C_{2}$ tal que:

(i) $\tilde{\omega}_{k}$ é suficientemente negativo, $\tilde{\omega}_{i}=0$ para todo $i \neq k$ e $\tilde{W}_{j}=0$ para $j=1, \ldots, s$ ou

(ii) $\tilde{\omega}_{i}=0$, para $i=1, \ldots, r,\left\|W_{l}\right\|_{F}$ é suficientemente grande e $\tilde{W}_{j}=0$ para todo $j \neq l$.

Portanto, $\lambda \geq 0$ e $U_{j} \succeq 0$ para $j=1, \ldots, s$. Além disso, a partir da inequação (B.5b) e do fato de que $(0, \ldots, 0,0, \ldots, 0) \in C_{2}$, é fácil ver que $\eta \geq 0$. Então, por (B.5a), para todo elemento $\left(\nu_{1}, \ldots, \nu_{r}, V_{1}, \ldots, V_{s}\right) \in C_{1}$, temos que

$$
0 \leq \sum_{i=1}^{r} \lambda_{i} \nu_{i}+\left\langle U_{1}, V_{1}\right\rangle+\cdots+\left\langle U_{s}, V_{s}\right\rangle .
$$


Chamamos de $D$ o conjunto $C \cap \operatorname{dom} f_{1} \cap \cdots \cap \operatorname{dom} f_{r}$. Para todo $x \in D$ e todo $\epsilon>0$, sabemos que $\left(f_{1}(x)+\epsilon, \ldots, f_{r}(x)+\epsilon, G_{1}(x)+\epsilon I, \ldots, G_{s}(x)+\epsilon I\right) \in C_{1}$. Assim, pela expressão (B.6), para todo $x \in D$ e todo $\epsilon>0$, temos

$$
0 \leq \lambda_{1}\left(f_{1}(x)+\epsilon\right)+\cdots+\lambda_{r}\left(f_{r}(x)+\epsilon\right)+\left\langle U_{1}, G_{1}(x)+\epsilon I\right\rangle+\cdots+\left\langle U_{s}, G_{s}(x)+\epsilon I\right\rangle,
$$

resultando que

$$
0 \leq \lambda_{1} f_{1}(x)+\cdots+\lambda_{r} f_{r}(x)+\left\langle U_{1}, G_{1}(x)\right\rangle+\cdots+\left\langle U_{s}, G_{s}(x)\right\rangle, \quad \forall x \in D .
$$

Por hipótese, $\operatorname{dom} f_{i} \supseteq \operatorname{ri} C$ para $i=1, \ldots, r$. Logo, $D \supseteq$ ri $C$ e, conseqüentemente, a inequação (B.7) é válida para todo $x \in \operatorname{ri} C$. Assim, pelo Teorema 7.3.3 de [Roc70], sabemos que a inequação (B.7) é válida para todo $x \in \operatorname{cl}(\operatorname{ri} C)=\operatorname{cl} C \supseteq C$, o que encerra a prova. 


\section{Referências Bibliográficas}

[ABMS05a] R. Andreani, E. G. Birgin, J. M. Martínez, and M. L. Schuverdt. Augmented lagrangian methods under the constant positive linear dependence constraint qualification. Mathematical Programming, to appear, 2005.

[ABMS05b] R. Andreani, E. G. Birgin, J. M. Martínez, and M. L. Schuverdt. On augmented lagrangian methods with general lower-level constraints. Technical Report MCDO-050304, Department of Applied Mathematics, UNICAMP, Brazil, 2005 .

[Bar76] R. G. Bartle. The elements of real analysis. John Wiley \& Sons, New YorkLondon-Sydney, 2nd edition, 1976.

[Ber82] D. P. Bertsekas. Constrained optimization and Lagrange multiplier methods. Computer Science and Applied Mathematics. Academic Press Inc. [Harcourt Brace Jovanovich Publishers], New York, 1982.

[Ber99] D. P. Bertsekas. Nonlinear Programming. Athena Scientific, Belmont, MA, 2nd edition, 1999.

[Ber03] D. P. Bertsekas. Convex analysis and optimization. Athena Scientific, Belmont, MA, 2003. With Angelia Nedić and Asuman E. Ozdaglar.

[BL06] Jonathan M. Borwein and Adrian S. Lewis. Convex analysis and nonlinear optimization. CMS Books in Mathematics/Ouvrages de Mathématiques de la SMC, 3. Springer, New York, second edition, 2006. Theory and examples.

[BM02] Ernesto G. Birgin and José Mario Martínez. Large-scale active-set boxconstrained optimization method with spectral projected gradients. Comput. Optim. Appl., 23(1):101-125, 2002.

[Bor99] B. Borchers. SDPLIB 1.2, a library of semidefinite pogramming test problems. Optim. Methods Softw., 11/12(1-4):683-690, 1999.

[BTN01] A. Ben-Tal and A. Nemirovski. Lectures on modern convex optimization. MPS/SIAM Series on Optimization. Society for Industrial and Applied Mathematics (SIAM), Philadelphia, PA, 2001. Analysis, algorithms, and engineering applications.

[BTZ97] A. Ben-Tal and M. Zibulevsky. Penalty/barrier multiplier methods for convex programming problems. SIAM J. Optim., 7(2):347-366, 1997. 
[DM02] Elizabeth D. Dolan and Jorge J. Moré. Benchmarking optimization software with perfomance profiles. Mathematical Programming, 91:201-213, 2002.

[DT99] M. Doljansky and M. Teboulle. An interior proximal algorithm and the exponential multiplier method for semidefinite programming. SIAM J. Optim., 9(1):1-13 (electronic), 1999.

[Eck03] Jonathan Eckstein. A practical general approximation criterion for methods of multipliers based on bregman distances. Mathematical Programming, 96(1):6186, 2003.

$\left[\mathrm{FFK}^{+} 08\right]$ Katsuki Fujisawa, Mituhiro Fukuda, Kazuhiro Kobayashi, Masakazu Kojima, Kazuhide Nakata, Maho Nakata, and Makoto Yamashita. SDPA (semidefinite programming algorithm) - user's manual - version 7.0.5. Research Report B448, Tokyo Institute of Technology, February 2008.

[GVL96] Gene H. Golub and Charles F. Van Loan. Matrix computations. Johns Hopkins Studies in the Mathematical Sciences. Johns Hopkins University Press, Baltimore, MD, third edition, 1996.

[Hes69] M.R. Hestenes. Mulpliers and gradient methods. Journal on Optimization Theory and Applications, 4:303-320, 1969.

[HJ90] R. A. Horn and C. R. Johnson. Matrix analysis. Cambridge University Press, Cambridge, 1990. Corrected reprint of the 1985 original.

[HJ94] R. A. Horn and C. R. Johnson. Topics in matrix analysis. Cambridge University Press, Cambridge, 1994. Corrected reprint of the 1991 original.

[KS01] Michal Kočvara and Michael Stingl. PENNON - a generalized augmented Lagrangian method for semidefinite programming. In G. Di Pillo and A. Murli, editors, High Performance Algorithms and Software for Nonlinear Programming, pages 297-315. Kluwer Academic Publishers, 2001.

[KS03] Michal Kočvara and Michael Stingl. PENNON: a code for convex nonlinear and semidefinite programming. Optimization Methods and Software, 18(3):317$333,2003$.

[Lew96] A. S. Lewis. Convex analysis on the Hermitian matrices. SIAM J. Optim., 6(1):164-177, 1996.

[Man69] O. L. Mangasarian. Nonlinear Programming. McGraw-Hill Book Company, New York, 1969.

[MZ00] L. Mosheyev and M. Zibulevsky. Penalty/barrier multiplier algorithm for semidefinite programming. Optim. Methods Softw., 13(4):235-261, 2000.

[Pow69] M.J.D. Powell. A method for nonlinear contraints in minimization problems. In R. Fletcher, editor, Optimization, pages 283-298. Academic Press, 1969. 
[Roc70] R. T. Rockafellar. Convex analysis. Princeton Mathematical Series, No. 28. Princeton University Press, Princeton, N.J., 1970.

[See97] A. Seeger. Convex analysis of spectrally defined matrix functions. SIAM J. Optim., 7(3):679-696, 1997.

[Sil00] P. Silva. Tópicos em Métodos de Ponto Proximal. PhD thesis, Universidade de São Paulo, USP, São Paulo, Brasil, 2000.

[Sil06] R. Silva. Trajetória Central em Programação Semidefinida, Método de Ponto Proximal Generalizado e Trajetória de Cauchy em Variedade Riemanniana. $\mathrm{PhD}$ thesis, Universidade Federal do Rio de Janeiro, UFRJ, Rio de Janeiro, Brasil, 2006.

[TB97] Lloyd N. Trefethen and David Bau, III. Numerical linear algebra. Society for Industrial and Applied Mathematics (SIAM), Philadelphia, PA, 1997.

[Tod01] M. J. Todd. Semidefinite optimization. Acta Numer., 10:515-560, 2001.

[WSV00] Henry Wolkowicz, Romesh Saigal, and Lieven Vandenberghe, editors. Handbook of semidefinite programming. International Series in Operations Research \& Management Science, 27. Kluwer Academic Publishers, Boston, MA, 2000. Theory, algorithms, and applications. 